\title{
Fluid Mechanics of chemical and flow sensing in aquatic animals
}

\footnotetext{
A Dissertation

Presented to

the Faculty of the School of Engineering and Applied Science

University of Virginia

In Partial Fulfillment

of the requirements for the Degree

Doctor of Philosophy (Mechanical and Aerospace Engineering)

by

Swapnil Pravin

December 2014
} 
(c) Copyright by Swapnil Pravin

All rights reserved

December 2014 


\section{Approval Sheet}

This dissertation is submitted in partial fulfillment of the requirements for the degree of Doctor of Philosophy (Mechanical and Aerospace Engineering)

Swapnil Pravin

This dissertation has been read and approved by the Examining Committee:

Matthew Reidenbach, Adviser

Houston Wood, Committee Chair

Deforest Mellon, Jr.

Hossein Haj-Hariri

Haibo Dong

Accepted for the School of Engineering and Applied Science:

Dean, School of Engineering and Applied Science

December 2014 


\section{Abstract}

Crustaceans such as crabs, lobsters and crayfish use chemical sensing to determine the location of predators, prey, potential mates and habitat. Chemical signals are primarily distributed throughout terrestrial and aquatic environments by convective and diffusive transport processes that are affected by turbulence. Many animals actively sample odorbearing fluid using appendages bearing arrays of hair-like chemosensory and mechanosensory sensilla. These animals sample their surrounding odor and fluid environment by flicking their appendages, essentially taking a "sniff". A comprehensive understanding of the chemosensory system in these animals requires examination of the morphology of olfactory appendages, kinematics of flicking behavior and the nature of flow and odorant distribution in the aquatic environments around them. Chemosensing in animals also has the potential to inspire the design of artificial chemical sensors. Numerical modeling and examination of experimental data were combined to examine the nature of turbulent benthic flows and olfactory systems in animals. A numerical model was developed to determine advective-diffusive transport of odorant molecules to olfactory appendages of the crayfish, Procambarus clarkii. I tested the extent of molecule transport to the surfaces of aesthetasc sensilla during an antennule flick and the degree of odorant exchange during subsequent flicks. Odorant molecules were advected between the aesthetascs during the rapid downstroke of the flick and were trapped between the sensilla during the return stroke. Up to $97.6 \%$ of these odorants are replaced with new odorant molecules during subsequent flicks. The concentration of molecules captured along aesthetasc surfaces was found to increase with increased gap spacing between 
aesthetascs, flick speed, and distance from the proximal end of the aesthetasc. Secondly, to determine how simultaneous flow and odorant sampling can aid in search behavior, a three-dimensional numerical model for the near-bed flow environment was created. A stream of odorant concentration was released into the flow creating a turbulent plume, and both temporally and spatially fluctuating velocity and odorant concentration were quantified. Odorant fluxes measured transverse to the mean flow direction, quantified as the product of the instantaneous fluctuation in concentration and velocity, $v^{\prime} c^{\prime}$, show statistically distinct magnitude and directional information on either side of a plume centerline over integration times of $<0.5 \mathrm{~s}$. Aquatic animals typically have neural responses to odorant and velocity fields at rates between 50 and $500 \mathrm{~ms}$, suggesting this simultaneous sampling of both flow and concentration in a turbulent plume can aid in source tracking on timescales relevant to aquatic animals. Lastly, the hydrodynamic sensory system and its dependence on sensilla morphology was studied. Numerical models of four predominant sensilla morphologies were simulated to quantify their deformation under the pressure of the incoming flow as a result of upstream flow perturbation. Results show that peaks in velocity and pressure at the sensilla are caused by the upstream perturbation. The standing feathered sensilla are found to experience the maximum force, bending moment and deformation among the tested sensilla morphologies. 
In dedication to my mother 


\section{Acknowledgements}

First and foremost, I offer my sincerest gratitude to my advisor Matthew Reidenbach for his continuous support in my Ph.D. study and research, for his patience, encouragement, motivation and guidance. My time in graduate school would not have been so enriching without him. Pepe Humphrey was a great mentor and advisor who helped tremendously in my transition to graduate school. His care and affection will stay forever with me. Mike Mellon has been a wonderful mentor and has helped me learn so much about crustacean neurophysiology. I would also like to thank the rest of my dissertation committee: Haibo Dong, Hossein Haj-Hariri and my dissertation chair, Houston Wood, for all of their helpful comments, suggestions and the effort they put into this dissertation.

My time at the university has been blessed with a friendly and cheerful lab group. Jonathan Stocking has been a constant source of humor, camaraderie, support and knowledge. I reflect fondly on my interactions with Ross Timmerman who was always a helping and cheerful presence in the lab. I also have to thank Amanda Timmerman for making numerous experiments in the lab possible. Without your help, I could never have managed to make those crayfish cooperate in our experiments. Emily Thomas and Elizabeth Murphy were always great sources of helpful ideas and suggestions. Outside of the lab, Kulwant Rai, who was one of the first people I met in Charlottesville, has been and continues to be a source of encouragement and a dear friend. I also thank Arunvel Kailasan for his continuous support and being a source of inspiration. Our lunches will be a cherished part of my time here. 
Finally, I thank my parents Sunita and Pravin Kumar for supporting me throughout all my studies and my sister Swati for her constant encouragement and love. 


\section{Contents}

Contents vii

List of Tables . . . . . . . . . . . . . . . . . . $\quad$ x

List of Figures . . . . . . . . . . . . . . . . . . . xi

Nomenclature $\quad$ xix

1 Introduction $\quad 1$

1.1 Research Questions . . . . . . . . . . . . . . . . . . . 4

1.2 Dissertation Roadmap . . . . . . . . . . . . . . . . . . 4

2 Micro-scale fluid and odorant transport to antennules of the crayfish, Pro$\begin{array}{ll}\text { cambarus clarkii } & 6\end{array}$

2.1 Introduction . . . . . . . . . . . . . . . . . . . . 7

2.2 Methods . . . . . . . . . . . . . . . . . . 10

2.2.1 Model independence . . . . . . . . . . . . . . . . 15

2.3 Results . . . . . . . . . . . . . . . . . . . . . . . . . . . . . . . . . . . . . . .

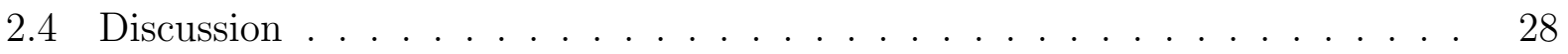

2.4.1 Aesthetasc spacing and length . . . . . . . . . . . . . 30

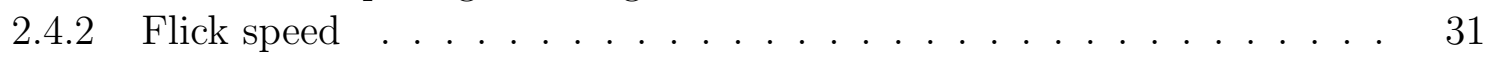

2.4.3 Odorant filament width . . . . . . . . . . . . . . . . . 32

2.4.4 Conclusions regarding antennule flicking and odorant detection by $P$. clarkii .......................... 32

3 Simultaneous sampling of flow and odorants by crustaceans can aid search within a turbulent plume $\quad 35$

3.1 Introduction . . . . . . . . . . . . . . . . . 36

3.1.1 Chemosensing in Aquatic Animals . . . . . . . . . . . . . . . 36

3.1.2 Sampling Rates and Tracking Strategies in Crustaceans . . . . . . . . 38

3.1.3 Neural Responses to Odorant and Flow Information . . . . . . . . . . 39

3.1.4 Research Objectives ................... . . 41

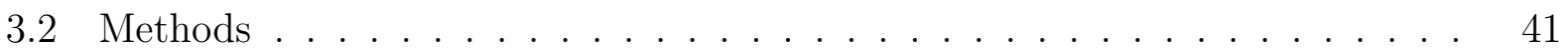

3.2.1 Flume Study . . . . . . . . . . . . . . . . . . . . . . . . . . . . . . . . 41

3.2.2 Numerical Model . . . . . . . . . . . . . . . . . . . . . . . . 44

3.2.3 Mesh convergence . . . . . . . . . . . . . . . . . 47

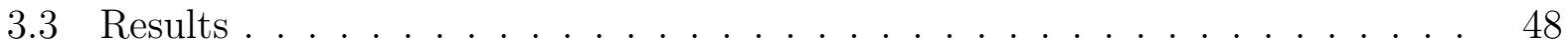


3.3.1 Laboratory Flume Studies . . . . . . . . . . . . . . . . . . . . . 48

3.3 .2 Numerical Model . . . . . . . . . . . . . . . . . . . . . . . . . . . . . 49

3.3.3 Temporal and Spatial Averaging of the Plume . . . . . . . . . . 53

3.4 Discussion . . . . . . . . . . . . . . . . . . 56

3.4.1 Neural Responses to Odorants and Flows . . . . . . . . . . . . . 59

3.5 Conclusions . . . . . . . . . . . . . . . . . . . 60

4 Effects of sensilla morphology on mechanosensory sensitivity in crustaceans 62

4.1 Introduction . . . . . . . . . . . . . . . . . . . 63

4.1.1 Antennule flicking and mechanoreception in crustaceans . . . . . . . 64

4.1.2 Simultaneous odorant and flow sensing . . . . . . . . . . . . 66

4.1 .3 Research objectives . . . . . . . . . . . . . . . . 66

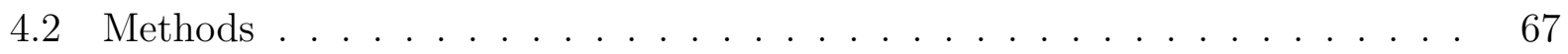

4.2 .1 Mechanosensory sensilla morphologies . . . . . . . . . . . 67

4.2 .2 Upstream flow perturbation response . . . . . . . . . . . . . . . 69

4.2 .3 Sensilla bending analysis . . . . . . . . . . . . . . . . . . 72

4.2 .4 Computation of torsional stiffness . . . . . . . . . . . . . . . 73

4.3 Results . . . . . . . . . . . . . . . . . . . . . . . . . . 74

4.3.1 Upstream perturbation response . . . . . . . . . . . . . . . . . 74

4.3 .2 Sensilla response to perturbed flow . . . . . . . . . . . . . 75

4.3.3 Direction of perturbation . . . . . . . . . . . . . . 76

4.3.4 Model sensitivity to torsional stiffness . . . . . . . . . . . . 76

4.4 Discussion . . . . . . . . . . . . . . . . . . . . . . 77

4.4 .1 Sensilla morphology and sensitivity . . . . . . . . . . . . . . . . . 79

4.4 .2 Relative sensilla sensitivity and function . . . . . . . . . . . 81

4.4.3 Role of hydrodynamic sensing in plume tracking . . . . . . . . . . 84

4.4.4 Sensitivity to direction of perturbation . . . . . . . . . . 85

$\begin{array}{llr}5 & \text { Conclusions } & 88\end{array}$

$\begin{array}{ll}\text { Appendices } & 91\end{array}$

A A nose too far: regional differences in olfactory receptor neuron efficacy $\begin{array}{lr}\text { along the crayfish antennule } & 92\end{array}$

A.1 Introduction . . . . . . . . . . . . . . . . . 93

A.2 Materials and Methods . . . . . . . . . . . . . . . 95

A.3 Results . . . . . . . . . . . . . . . . . . . . . . . . . 99

A.3.1 Antennular anatomy and aesthetasc distribution . . . . . . . . . 99

A.3.2 Neurophysiological observations . . . . . . . . . . . . . . . . 102

A.4 Discussion . . . . . . . . . . . . . . . . . . . . . . . 108

A.5 Acknowledgments . . . . . . . . . . . . . . . . . . 112

$\begin{array}{ll}\text { B CFD model of antennule housing chamber } & 113\end{array}$ 
C Odorant diffusion over rough topography - A two-dimensional study 115

C.1 Introduction . . . . . . . . . . . . . . . . . . . . 115

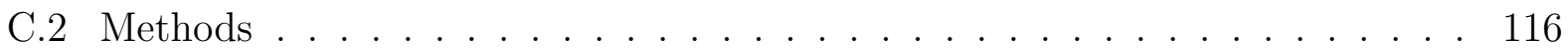

C.3 Results . . . . . . . . . . . . . . . . . . . . 117

$\begin{array}{ll}\text { Bibliography } & 122\end{array}$ 


\section{List of Tables}

2.1 Values of $T_{1}$ and $T_{2}$ (the time instants that signify the temporal bounds of an odorant filament as shown in Fig. 10) for filaments encountered at the beginning of a flick. . . . . . . . . . . . . . . .

2.2 Values of $T_{1}$ and $T_{2}$ (the time instants that signify the temporal bounds of an odorant filament as shown in Fig. 10) for filaments encountered at the end of a flick. . . . . . . . . . . . . . . . . . . . .

3.1 Mean statistics for the simulated flow at inlet flow speeds of $U_{0}=10$ and 15 $\mathrm{cm} \mathrm{s}^{-1}$ respectively. $U_{0}$ is the inlet velocity along the upstream end of the flow domain. $\bar{u}$ is the mean velocity over the bed roughness $1 \mathrm{~m}$ downstream from the source, $u_{*}$ is the friction velocity, $R e_{*}$ is the roughness Reynolds number computed as $R e_{*}=u_{*} h / \nu$ ( $\mathrm{h}=$ height of roughness element), $\eta$ is the Kolmogorov lengthscale, $\epsilon$ is the dissipation rate of turbulence and $\lambda_{B}$ is the Batchelor scale. . . . . . . . . . . . . . . . . . .

4.1 Dimensions and properties of various morphologies of sensilla found on a crayfish antennule . . . . . . . . . . . . . . . . 
A.1 Spiking responses to zone - and zonal combination stimulation by odorant. Mean spike numbers (APs) \pm 1 S.E. (SE) in response to stimulation of different numbers of aesthetascs (Aes) occurring within single zones or zonal combinations in five preparations, identified by bold-numbered dates above the columns, in addition to those graphed in Figure A.7(A) Mean response coefficients obtained from Type I local olfactory interneurons in fourteen preparations to separate stimulation via the three stimulus ports. Zone one, nearest the proximal origin of the aesthetasc array, consistently elicited the most vigorous responses to standard odorant exposures. (B) Graph of response coefficients in local olfactory interneurons from the same fourteen different preparations against $\mathrm{Z}$, a dimensionless variable which varies between 0 and 1 as a function of distance along the aesthetasc array from its origin to its termination at the flagellum tip. Responses from each preparation are represented here by a different colored symbol; data points in most cases are the mean of 5-10 stimulus trials \pm 1 S.E. The data are satisfactorily fit by a first-order exponential decay curve defined by the equation $Y=$ $6.67 e^{-X / 21}+0.27$. Nonlinear regression analysis on this functional model yielded $r^{2}=0.6$ and $\mathrm{P}<0.0001$.figure.caption.67. Pearsons $\mathrm{r}$ values were obtained by linear regression analysis. Values of $\mathrm{P}$ were calculated using software at www.danielsoper.com/statcalc3/calc.aspx?id=44 . . . . . . 106 


\section{List of Figures}

2.1 (a) Antennular flagellum of Procambarus clarkii. Grid in background is $1 \mathrm{~cm}$ by $1 \mathrm{~cm}$. (b) Annuli of an antennule of P. clarkii. Aesthetasc sensilla (shown by black arrows) are arrayed ventrally on each annulus of the distal half of the flagellum . . . . . . . . . . . . . . . . . . .

2.2 Schematics of flow past the antennule and aesthetascs . . . . . . . . .

2.3 (a) Velocity and (b) odorant concentration at inlet boundary. Both the quantities are assumed to have a parabolic time dependent influx to represent the flicking motion of the antennule. $t_{1}=0.118 \mathrm{~s}$ represents the end of downstroke and $t_{2}=0.297 \mathrm{~s}$ represents the end of the entire forward-return sequence, as measured from video recordings of antennule flicking by adult P. clarkii . . .

2.4 Mesh grid cells around the aesthetascs and antennule. Cell size adjacent to the aesthetasc surface is $0.15 \mu \mathrm{m}$ and that around the antennule surface is $5 \mu \mathrm{m}$

2.5 Flow velocity between the aesthetascs over the course of a flick for different sizes of the flow domain. . . . . . . . . . . . . . . . . . .

2.6 Concentration contours (normalized by $C_{\infty}$ ) at the end of (a) downstroke and (b) returnstroke, and zoomed-in images of concentration surrounding the aesthetascs at the end of the (c) downstroke and (d) returnstroke. A region of trapped odorant molecules between the aesthetascs can be seen at the end of return stroke. . . . . . . . . . . . . . . . . . . . .

2.7 Normalized odorant concentration at four different locations on the surface of an aesthetasc during a downstroke/returnstroke flick sequence. For this aesthetasc geometry, $\theta=180^{\circ}$ represents the location on the aesthetasc that faces the ambient flow, while $\theta=270^{\circ}$ represents the location on the aesthetasc that faces the other aesthetasc. . . . . . . . . . . . . . . .

2.8 Rate of normalized concentration change at four different locations on the surface of an aesthetasc during a downstroke/returnstroke flick sequence. For this aesthetasc geometry, $\theta=180^{\circ}$ represents the location on the aesthetasc that faces the ambient flow, while $\theta=270^{\circ}$ represents the location on the aesthetasc that faces the other aesthetasc. . . . . . . . . . . . . .

2.9 Concentration at aesthetasc surface for a second flick following an initial flick. Components of total odorant concentration are new odorant molecules acquired in the second flick and the old molecules remaining from the initial flick. . 
2.10 Illustration of an antennule at various stages of a downstroke as it passes through an odorant filament. The shaded region shows the area that constitutes the odorant. The antennule starts (a) in front of the filament, (b) gets completely immersed in the filament and (c) traverses through the odorant filament towards the end of the downstroke. This sequence is repeated in reverse order as the antennule comes back through the filament during the return stroke . . . . . . . . . . . . . . . . . . . .

2.11 Boundary conditions for (a) velocity and (b) odorant concentration for an odorant filament placed at the start of a downstroke. The solid lines and the shaded region show the portion of the flick that is spent by the antennule inside the filament. Odorant filaments grow thicker as both $T_{1}$ and $T_{2}$ move closer to $t_{1} \ldots \ldots \ldots \ldots \ldots \ldots \ldots$

2.12 Variation of normalized mean concentration of odorant molecules around the aesthetasc surface with (a) odorant filaments of various width encountered during the beginning of the downstroke motion of a flick, (b) odor filaments of various width encountered during the end of the downstroke, (c) aesthetasc spacing, (d) flick speed, and (e) position of sensing at different distal distances along the length of an aesthetasc. Boxplots indicate the variation of odorant concentration transported to the surface of the aesthetasc. Horizontal line within the box indicates the median, while the lower and upper edges of the box represent the 25 th and 75 th percentiles, respectively. Vertical lines extending from the box indicate the minimum and maximum measured values. . . . . .

2.13 Flushing efficiency, quantified as the fraction of old molecules displaced by new molecules during subsequent flicks, plotted in relation to (a) aesthetasc spacing, (b) flick speed, and (c) position of sensing at different distal distances along the length of an aesthetasc. Boxplots are described in figure 2.12Variation of normalized mean concentration of odorant molecules around the aesthetasc surface with (a) odorant filaments of various width encountered during the beginning of the downstroke motion of a flick, (b) odor filaments of various width encountered during the end of the downstroke, (c) aesthetasc spacing, (d) flick speed, and (e) position of sensing at different distal distances along the length of an aesthetasc. Boxplots indicate the variation of odorant concentration transported to the surface of the aesthetasc. Horizontal line within the box indicates the median, while the lower and upper edges of the box represent the 25th and 75th percentiles, respectively. Vertical lines extending from the box indicate the minimum and maximum measured values.figure.caption.26. Smaller boxplots indicate more uniform concentrations sampled along the circumference of the aesthetasc. . . . . . . . . . . . . . . . . . .

2.14 Variation of normalized odorant concentration with time during a flick-return sequence for different values of $g / d$ at $v=v_{0} . g / d$ ratios for each line are labeled on the figure. $\left(d\right.$ is the typical gap between the aesthetascs and $v_{0}$ is the typical flick speed.) . . . . . . . . . . . . . . . . . . .

2.15 Variation of normalized odorant concentration with time during a flick-return sequence for different flick speeds at $g / d=1 . v / v_{0}$ ratios for each line are labeled on the figure. . . . . . . . . . . . . . . . . . 
3.1 (A) The freshwater crayfish, Procambarus clarkii, with lateral antennule labeled. Grid in the background is $1 \times 1 \mathrm{~cm}$; (B) Scanning electron micrograph (SEM) of the lateral antennule with (a) chemosensory aesthetascs and (b) mechanosensory sensilla labeled (photo D. Mellon); (C) The spiny lobster, Panulirus argus, with the lateral antennule labeled; (D) SEM of the lateral antennule with (a) chemosensory aesthetascs; (b) mechanosensory sensilla and (c) guard hairs labeled (photo J.A. Goldman). . . . . . . . . . . . . . . . .

3.2 Recirculating flume with PLIF/PIV laser system. A $532 \mathrm{~nm}$ laser was used for particle image velocimetry, while a $488 \mathrm{~nm}$ laser was used to excite fluorescein dye for use in PLIF imaging. Images were obtained $13 \mathrm{~m}$ downstream from the leading edge of the flume and $1 \mathrm{~m}$ downstream from the source release of fluorescein dye. . . . . . . . . . . . . . . . . . . .

3.3 Schematics of the CFD domain for flow simulation. The bottom surface of the three-dimensional domain has cubical roughness elements placed in a staggered fashion. Water enters from the left end and exits from the right. The roughness humps at the bottom, of side length $1 \mathrm{~cm}$ and separated by $5 \mathrm{~cm}$ from each other, help trip the boundary layer and facilitate mixing leading to a turbulent flow downstream. A stream of odorants is released into the inflow at the odorant inlet on the left at an elevation of $z=0.5 \mathrm{~cm}$. The cubical humps have a base area of $1 \mathrm{~cm}$ by $1 \mathrm{~cm}$ and a height of $0.5 \mathrm{~cm}$ to hydraulically reproduce a sand bed roughness. . . . . . . . . . . . . . . . .

3.4 Outlet flow velocity profile over the height of the flume column for different mesh resolutions. A mesh size of 3.3 million elements is used for the flume model. . . . . . . . . . . . . . . . . . . . .

3.5 (A) Image of particle-laden fluid used in the PIV analysis; (B) PLIF image of plume structure $1 \mathrm{~m}$ downstream of the source; $(\mathrm{C})$ combined plume structure from PLIF and velocity vectors (shown as blue arrows) calculated from PIV cross-correlation analysis. Odorant was released $1 \mathrm{~m}$ upstream from the imaging area by emitting fluorescein dye from a $1 \mathrm{~cm}$ tygon tube embedded across the width of the flume in the sandy bed material. Flow is from left to right. . . . . . . . . . . . . . . . . . .

3.6 (A) Mean and fluctuating components of horizontal velocity within the laboratory flume from PIV. Fluctuating component computed as the root mean square (rms) of the horizontal velocity; (B) Mean and rms fluctuating component of normalized concentration from PLIF; (C) Mean horizontal turbulent flux $\left(\mathrm{g} \mathrm{cm}^{-2} \mathrm{~s}^{-1}\right)$, computed as mean of the fluctuating horizontal velocity, $u^{\prime}$, (in $\mathrm{cm} \mathrm{s}^{-1}$ ), multiplied by the fluctuating normalized concentration, $c^{\prime}$. . . .

3.7 (A) Side view of odorant concentration throughout the numerical flume. White squares are roughness elements along the bed; (B) Top-down view of odorant concentrations at an elevation of $z=0.5 \mathrm{~cm}$. The plume of odorants is released into the flume through a circular point-source inlet located at $z=0.3 \mathrm{~cm}$ above the bed. . . . . . . . . . . . . . . . . . . . .

3.8 Comparison of horizontal turbulence intensity within the flume experiments and numerical model. Measurements were obtained at $1 \mathrm{~m}$ downstream from the source. . . . . . . . . . . . . . . . . . . . 
3.9 (A) Instantaneous (A) odorant concentration; (B) horizontal velocity; and (C) transverse concentration flux $\left(v^{\prime} c^{\prime}\right)$ at $z=1 \mathrm{~cm}$ above the flume bed, obtained at $t=10 \mathrm{~s}$ after initial release of odorant. . . . . . . . . . . . .

3.10 Instantaneous (A) odorant concentration; (B) horizontal velocity; and (C) transverse concentration flux $\left(v^{\prime} c^{\prime}\right)$ at $z=3 \mathrm{~cm}$ above the flume bed, obtained at $t=10 \mathrm{~s}$ after initial release of odorant. . . . . . . . . . .

3.11 (A) Instantaneous (A) odorant concentration; (B) horizontal velocity; and (C) transverse concentration flux $\left(v^{\prime} c^{\prime}\right)$ at $z=5 \mathrm{~cm}$ above the flume bed, obtained at $t=10 \mathrm{~s}$ after initial release of odorant. . . . . . . . . . . .

3.12 (A) Normalized concentration; (B) horizontal velocity, $u$; and (C) transverse flux $\left(v^{\prime} c^{\prime}\right)$ at points a and b (as indicated in Figure 3.11(A) Instantaneous (A) odorant concentration; (B) horizontal velocity; and (C) transverse concentration flux $\left(v^{\prime} c^{\prime}\right)$ at $z=5 \mathrm{~cm}$ above the flume bed, obtained at $t=10 \mathrm{~s}$ after initial release of odorant.figure.caption.42C) for a $1 \mathrm{~s}$ time period. The solid lines show the cumulative mean and the shaded regions show the \pm 1 standard error around the cumulative mean. . . . . . . . . . . . . . . .

3.13 (A) Normalized concentration and (B) temporal rate of change of concentration $(d c / d t)$ along the centerline through the length of the flume at $z=5 \mathrm{~cm}$. The solid line shows mean values and the shaded region shows the region of \pm 1 standard deviation around the mean. . . . . . . . . . . . . . .

4.1 A. Antennular flagellum of Procambarus clarkii. B. SEM image of the filamentous sensilli C. SEM image of the beaked sensillum in background, filamentous sensillum in middle, and asymmetric sensillum in foreground. D. Feathered sensillum, (image modified from Mellon and Hamid [32]). Scale bar is $20 \mu \mathrm{m}$.

4.2 Idealized CAD models of four primary morphologies of sensilla found on crayfish antennules: (A) Feathered, (B) Beaked, (C) Filamentous and (D) Asymmetric sensilla. The scale bars are $25 \mu \mathrm{m} . \quad$. . . . . . . . . . . . . . .

4.3 CFD model for velocity and pressure response at the surface of antennule B $(d=0.5 \mathrm{~mm})$ caused by a pulse movement of the upstream circle A $(D=9.5 \mathrm{~mm})$. The graph shows the pulse displacement of circle $\mathrm{A}$ and the velocity and pressure profile at the sensilla attached to the antennule (circle B) at angle $\theta$ are used as inlet conditions for the sensilla CFD models. . . . . . . . . . .

4.4 (A) CFD model of the feathered sensilla. Inlet flow into the model is derived from the two-dimensional perturbation model. (B) A revolute joint at the proximal end of the sensilla allows it to rotate around the joint by an angle $\delta$ under the pressure of incoming flow. (C) Flowchart for computing the upstream fluid perturbation using the CFD model, which is then used to determine flow and pressure distribution around the sensilla. This pressure load is then inputted into the FSI model to compute angular deflection along the base of the sensilla. . . . . . . . . . . . . . . . . . . .

4.5 (A) Velocity and pressure response along the sensilla located on the downstream circle as the upstream sphere creates a pulse of $2 \mathrm{~ms}$ duration. Pressure contours (B) before and (C) after the pulse motion of the upstream circular entity. 
4.6 Flow velocity contours around the sensilla at two different heights from the base: A. asymmetric sensilla at $z=20$ and $60 \mu \mathrm{m}, \mathrm{B}$. beaked sensilla at $z=60$ and $140 \mu \mathrm{m}, \mathrm{C}$. feathered sensilla at $z=60$ and $120 \mu \mathrm{m}$ and D. filamentous sensilla at $z=70$ and $190 \mu \mathrm{m}$. The contours are colored by velocity and the sensilla surface is colored by magnitude of deformation of the sensilla from its original position. . . . . . . . . . . . . . . .

4.7 Angular deformation of the sensilla $\delta$, force exerted by the incoming flow and the corresponding bending moment for different sensilla morphologies: asymmetric, beaked, feathered and filamentous. . . . . . . . . .

4.8 Peak velocity of the incoming flow around the sensilla for various positions, $\theta$ around the circumference of the antennule. . . . . . . . . . .

4.9 Polar plot of normalized sensillar spiking thresholds in P. clarkii as a function of the relative position of a $5 \mathrm{~ms}$ near-field stimulus. Data are means \pm 1 s.e.m. of 25 sensilla. The 0 degree position was directly ahead of (distal to) the sensillum under observation. The stimulus probe was moved sequentially in 20 degree increments to 17 different positions clockwise and counterclockwise around the sensillum. (Adapted from Mellon and Hamid [32]) . . . . . . . .

4.10 Angular deformation, $\delta$ of the sensilla at positions around the antennule for different morphologies: A. asymmetric, B. beaked, C. feathered and D.

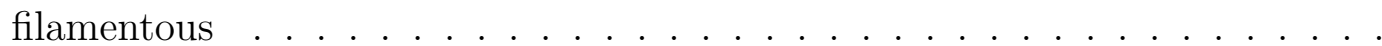

4.11 Sensitivity of sensilla deformation to variation in torsional stiffness, $k_{t}$ over several orders of magnitude. The AFM measurements and value used in the model are for $k_{t}=1 \times 10^{-12} \mathrm{~N} . \mathrm{m} /$ degree. The plot shows little variation in deformation $(\delta)$ for less stiff sensilla $\left(k_{t}<1 \times 10^{-12} \mathrm{~N} . \mathrm{m} /\right.$ degree$)$ but a sharp reduction in angular deformation, and thus sensitivity of the sensilla to hydrodynamic disturbance, for sensilla that have greater torsional stiffness.

A.1 (A) Diagram of the isolated head preparation and partitioned olfactometer used in the experimental procedures reported in this paper. The antennular flagella protrude through an aperture in the wall of the square recording chamber and into the olfactometer. Cannulas for brain perfusion and the recoding electrode placement are labelled "C". (B) detail of the partitioned olfactometer. Antennular flagella (not shown) protrude into the central horizontal chamber from the left and extend to the right beyond the most distal odorant port. Water enters the olfactometer chamber through the vertical port, while odorant enters through the three horizontal ports labeled "Odorant" and is removed via the ports labeled "Suction" after passing across the flagella. . . . . . . . .

A.2 Planar laser induced fluorescence images of dye within the central cylindrical chamber (denoted by dashed horizontal lines) where the antennule is located, sequentially applied through each of the three transversely aligned side channels (denoted by the dashed vertical lines). Concentration is normalized by input concentration and shows little mixing within the central chamber. . . . . . 
A.3 A, phase contrast micrograph of portion of a living lateral antennular flagellum from P. clarkii, modified from Mellon et al [9]. Aesthetasc sensilla are indicated by white arrows; each aesthetasc is supplied by the distal dendritic segments from approximately 175 ORNs, the cell bodies of which are clustered in individual sensory ganglia that are partially visible (asterisks) through the flagellar cuticle. B, light micrograph of a portion of a freshly excised lateral antennular flagellum from P. clarkii. The two antennular annuli included in the image (annulus borders indicated by white dashed lines) are from a region just distal to the origin of the aesthetasc array; each annulus in this region generally bears just a single aesthetasc (black arrows). Additional, numerous setae seen in micrographs B-D (black asterisks) are beaked sensilla [7] believed to serve a bi-modal mechano-chemosensory function). C, micrograph from the same lateral flagellum as in B, but from a region about $40 \%$ of the total length of the array distal to the origin $(z \sim 0.4)$. Here, each annulus is smaller than those near the array origin, and each bears three to four aesthetasc sensilla. $\mathrm{D}$, a portion of the same flagellum near the tip; aesthetascs often occur in clusters of three (arrows) at the proximal edge of an annulus and two at the distal edge, for a total of four to five per annulus, each of which is only about one-third the size of those shown in $\mathrm{C}$ and which, therefore, present greater numbers of aesthetascs exposed to the odorant stimuli at the port. All scale

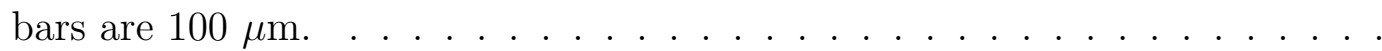

A.4 Distribution of aesthetasc number per annulus along the array in four freshly excised lateral antennular flagella (A and B are from opposite sides of the same crayfish, C and D are from two different crayfish) from animals in the range of 55-60 mm carapace length. The sensilla arrays, occupying between 32 and 42 flagellar annuli, are represented as dimensionless variables, $z$, where 0 is at the origin of the array, roughly half way distal from the base of the flagellum, and 1 is the termination of the array at the tip. The vertical rectangles in A approximate the position of ports one, two and three with respect to the aesthetasc array. While there is considerable variability between individual flagella, it is clear that the most proximal annuli harbor the fewest aesthetascs and that the density of aesthetascs per annulus increases distally. . . . . . .

A.5 Electrical records from a Type I neuron exemplifying responses to zonal exposure of the lateral flagellum to odorant. A-G, responses to $0.5 \mathrm{sec}$ odorant pulses (solid bars beneath electrical records) via the indicated ports or port combinations, occurring $2 \mathrm{sec}$ following the onset of water through the central olfactometer chamber). The gray rectangle delineates initial spikes generated by hydrodynamic inputs from the flagellum at the onset of water flow [68]. The number of aesthetascs exposed to odorant at port 1 was 1 , the number exposed at port 2 was 16, and the number exposed at port 3 was 17 . The $z$ position of the aesthetasc array at port 1 was 0 , that is, it was at the initial annulus of the array. Comparing the records in D-G with those in A-C, there is a clear indication of the additive relationship between central response and the extent of peripheral stimulation. . . . . . . . . . . . . . 
A.6 Spiking responses of three Type I interneurons to different numbers of stimulated aesthetascs, including those exposed by stimulus pulses in ports 1, 2, 3 and $1+2,1+3,2+3$ and $1+2+3$. The responses of individual Type I cells are indicated by the different colored symbols. The regression curve represents best linear fit of the combined data; it was calculated by least squares regression analysis and is represented by the equation $y=0.41 x+18.57$. Pearsons $r$ is 0.87 and $\mathrm{P}>0.0001 . \ldots \ldots \ldots \ldots$

A.7 (A) Mean response coefficients obtained from Type I local olfactory interneurons in fourteen preparations to separate stimulation via the three stimulus ports. Zone one, nearest the proximal origin of the aesthetasc array, consistently elicited the most vigorous responses to standard odorant exposures. (B) Graph of response coefficients in local olfactory interneurons from the same fourteen different preparations against Z, a dimensionless variable which varies between 0 and 1 as a function of distance along the aesthetasc array from its origin to its termination at the flagellum tip. Responses from each preparation are represented here by a different colored symbol; data points in most cases are the mean of 5-10 stimulus trials \pm 1 S.E. The data are satisfactorily fit by a first-order exponential decay curve defined by the equation $Y=6.67 e^{-X / .21}+0.27$. Nonlinear regression analysis on this functional model yielded $r^{2}=0.6$ and $\mathrm{P}<0.0001 \ldots \ldots \ldots \ldots$. . . . . . . . . .

B.1 Color-map of dye concentration throughout the recording chamber. The plot on the right shows odorant concentration along the centerline of the central channel. ................. . . .

C.1 Schematics of the 2D CFD model to solve for flow velocity and odorant concentrations throughout the flume. . . . . . . . . . . . . 116

C.2 Mesh for the CFD model . . . . . . . . . . . . . . . . . . . . . 116

C.3 Pressure, velocity and vorticity distributions around the roughness elements in the flume. Pressure units are Pascal and velocity units are m/s. . . . . . .

C.4 Odorant concentration color-map at a flow speed of $10 \mathrm{~cm} \mathrm{~s}^{-1}$. The units of odorant concentration are $\mathrm{kg} \mathrm{m}^{-3}$. . . . . . . . . . . . . . . . .

C.5 Time snapshot of odorant concentration along the length of the flume at a height of $5 \mathrm{~cm}$ from the bed. . . . . . . . . . . . . . . . . .

C.6 Correlation between concentrations at heights of $2 \mathrm{~mm}$ (very close to bed) and $5 \mathrm{~cm}$ (mid-way through the flume column). The odorant concentrations at these two heights are increasingly correlated as the animal moves away from the source owing to greater vertical mixing of the odorants. . . . . . . . . . . 120

C.7 Effect of flow speed of odorant concentration distribution throughout the flume.121 


\section{Nomenclature}

$c$ instantaneous chemical concentration.

$C_{\infty}$ ambient chemical concentration.

$c^{\prime}$ fluctuating chemical concentration.

$D$ molecular diffusivity.

$D_{R}$ eddy diffusivity.

$h$ height of roughness elements in the flume.

$k_{t}$ sensilla torsional stiffness.

$p$ pressure.

Re Reynolds number.

$R e_{*}$ roughness Reynolds number.

Sc Schmidt number $=\nu / D$.

$S_{i j}$ strain rate tensor.

$T_{1}$ time instant when the antennule exits an odorant filament during the downstroke.

$t_{1}$ time duration of downstroke of a flick. 
$T_{2}$ time instant when the antennule reenters an odorant filament during the returnstroke.

$t_{2}$ time duration of the entire forward and return flick motion.

$u$ instantaneous horizontal component of velocity.

$u_{i}$ instantaneous component of velocity in direction $\mathrm{i}$, where $\mathrm{i}=\mathrm{x}, \mathrm{y}, \mathrm{z}$.

$U_{p f}$ peak velocity for forward flick of antennule.

$U_{p r}$ peak velocity for return flick of antennule.

$u^{\prime}$ fluctuating horizontal component of velocity in downstream direction.

$u_{*}$ friction velocity.

$v^{\prime}$ fluctuating horizontal component of velocity in cross-stream direction.

$w^{\prime}$ fluctuating vertical component of velocity.

\section{Greek symbols}

$\epsilon$ turbulence dissipation rate.

$\eta$ Kolmogorov scale.

$\lambda_{B}$ Batchelor scale.

$\nu$ kinematic viscosity.

$\nu_{R}$ eddy viscosity.

$\rho$ density. 


\section{CHAPTER 1}

\section{Introduction}

Crustaceans such as crabs, lobsters and crayfish use chemo- and mechano-reception to track

sources of odorant plumes to locate mates, food, and living habitat $[1,2,3,4,5,6]$. Odorants in the benthic flow are carried to the olfactory organs of the animal through turbulent water currents and diffuse toward the surface of the organs where chemoreceptors are located. These olfactory organs also contain mechano-receptors that provide information about the turbulent flow, and together with odorant concentration help the animal locate the source of the chemical plume [7]. Animals use a variety of sensing strategies to orient themselves in the direction of the plume source depending on the flow regimes they operate in [8]. Hence, to understand the mechanism of chemical plume tracking in aquatic animals, we must understand not only the small scale diffusive flow of odorants near the olfactory organs of the animals, but also the large scale turbulent nature of the chemical plume. Crustaceans have olfactory appendages called antennules, which bear tiny hair-like structures called aesthetascs. The aesthetascs are often covered by a permeable cuticle membrane underneath which reside chemoreceptors. The chemoreceptors contained on the aesthetascs are composed of dendrites (branched projections) of olfactory receptor neurons (ORNs), which send information, through electrical impulses, to the olfactory lobes of the brain [9]. The diffusion of odorant molecules toward and through the cuticle membrane is responsible for delivering the odorants to these chemoreceptors. To 
facilitate the transport of odorant laden flow to the antennules, many aquatic animals use flicking or fanning of their appendages $[5,10]$. This behavior is often described as sniffing. A flicking motion often involves a fast down-stroke and a slower return-stroke, leading to entrapment of odorant molecules between the aesthetascs, which lowers the diffusion time of odorants to the aesthetasc surface and enables these animals to discretely sample their ambient environment $[11,12]$. Flicking in crustaceans has been shown to increase the fluid transport and the flux of odorants to aesthetasc surfaces $[11,13]$. Although other studies have quantified the importance of antennule flicking on the leakiness of air or water surrounding aesthetascs, few have quantified how this behavior impacts the advective-diffusive transport of odorants to aesthetasc surfaces. In addition, although trapping of fluid between adjacent aesthetascs has been studied in detail, discrete sampling of odorants has not. The odorant plumes encountered in the environment of these organisms are often turbulent and highly filamentous in nature $[6,14]$. Due to stirring by the turbulent motion of the fluid, the spatial and temporal distribution of odors is complex and filaments of high odor concentration are often adjacent to little or no odorants $[14,15]$. These distributions in odorants also change in response to variations in the ambient flow speed and bed roughness, where the variance in odorant fluctuations is reduced for rougher beds [16] and greater mean velocities [14]. Certain cues, such as correlations between the flow kinematics and odorant concentration that the animal sense through the chemoreceptors and mechanoreceptors, can provide valuable information regarding the plume source. However, due to the high intermittency and temporal and spatial variability of the plume, this often reduces the ability of organisms, such as the blue crab, Callinectes sapidus, to successfully navigate to the source of an attractive odor $[17]$.

Previous studies have shown that the time course of molecular diffusion of odorants to the chemoreceptors has a significant bearing on neural responses [18, 19, 20, 21], while others have argued that molecular diffusion does not play a limiting role in access of odorant molecules to chemoreceptors $[22,23,24]$. These hypotheses have been tested using calculations of 
odorant molecule flux to the aesthetasc surface based on assumptions regarding the near aesthetasc flow field $[25,26,27,28]$ or measurements of flow velocities surrounding aesthetascs utilizing particle-image velocimetry on dynamically scaled models [13, 29]. However, due to the exceedingly small scales at which transport occurs, $\mathrm{O}(\mu \mathrm{m})$, direct observations or measurements of odorant transport to aesthetasc surfaces have proven difficult.

Hydrodynamic stimulation of the lateral antennular flagellum has been found to evoke the flicking reflex in P. clarkii [30]. Reflex flicking is triggered by the standing feathered sensilla which are near-field receptors linearly arrayed along the antennular flagella [31]. Mellon and Hamid [32] confirmed that weak hydrodynamic stimuli are able to excite the feathered sensilla. They also present electrophysiological and behavioral evidence that standing feathered sensilla on the lateral antennular flagella, sensors that are very sensitive to directly imposed mechanical disturbances [31], respond to water particle motions. A rich diversity of receptors is found on the antennules of different crustaceans. Hydrodynamic stimulation of feathered sensilla shows that the sensilla are the most sensitive to impulsive stimuli originating close to the axial plane of the flagellum [32]. An examination of the SEM images of the sockets of a feathered sensilla reveals that the preferred directional sensitivities are at right angles to the hinge-like elongation of the sensillar base. Electrophysiological and behavioral studies show that both lateral and medial flagella respond to hydrodynamic cues [33]. Although the sensitivity of the sensilla to near field stimulus is established, the response of the various kinds sensilla structure to the stimulus is largely unknown.

A broad aim of this study is to provide insights into chemoreception mechanisms in aquatic animals by shedding lights on the physical mechanics of interaction of their olfactory organs with their fluid environment. This research also lays grounds for integration of fluid mechanics and chemical transport with neurophysiological response of the animal. The novel contribution of this research lies in quantifying chemical and fluid transport to a variety of olfactory organs of crustaceans under different flow conditions and their response to such stimulus. Providing greater understanding of turbulent odorant plumes and possible strategies 
for animals to navigate such environments is another novel contribution of this research.

\subsection{Research Questions}

The freshwater crayfish, Procambarus clarkii is used as the subject organism to study the sensilla morphology and flicking kinematics typically found in aquatic animals. Understanding the advection of odorants to the sensillar structure present on the animal at a small scale combined with characteristics of large-scale turbulent flow and odorant transport can help in understanding the driving mechanism behind the chemical and hydrodynamic sensing phenomenon in animals. The examination of various aspects of chemical sensing leads us to the following questions:

1. How does the morphology of aesthetasc sensilla and flicking kinematics effect odorant transport to the surface of the aesthetascs?

2. What is the impact of ambient flow environments on turbulent mixing and odorant transport within a chemical plume and can the animal conceivably integrate velocity and concentration information to aid in finding the location of the plume source?

3. How does hydrodynamic perturbation effect flow and pressure distribution near an organism and what is the effect of sensilla morphology on their relative sensitivity to upstream perturbations?

\subsection{Dissertation Roadmap}

The dissertation is divided into five chapters. Following the introduction in chapter 1, chapter 2 describes the numerical model of aesthetasc sensilla and micro-scale odorant transport to its surface. Chapter 3 contains the computational fluid dynamics (CFD) model study of a large flume with rough bed and turbulent odorant plume characteristics. Chapter 4 described the 
upstream perturbation study and the response of four major sensilla morphologies. Chapter 5 summarizes the conclusions of this research and makes recommendations for further study. Appendix A describes regional differences in olfactory receptor neuron efficacy along the crayfish antennule. Appendix B contains a CFD model of the test chamber used to house the antennule for stimulation experiments conducted in Appendix A. Appendix C contains a two-dimensional CFD model that describes odorant flow over a rough flume bed. 


\title{
CHAPTER 2
}

\section{Micro-scale fluid and odorant}

\section{transport to antennules of the}

\section{crayfish, Procambarus clarkii}

A part of this chapter has been published, with citation: Pravin, S., Mellon Jr, D., and Reidenbach, M. A. (2012). Micro-scale fluid and odorant transport to antennules of the crayfish, Procambarus clarkii. Journal of Comparative Physiology A, 198(9), 669-681.

\begin{abstract}
A numerical model was developed to determine advective-diffusive transport of odorant molecules to olfactory appendages of the crayfish, Procambarus clarkii. We tested the extent of molecule transport to the surfaces of aesthetasc sensilla during an antennule flick and the degree of odorant exchange during subsequent flicks. During the rapid downstroke of a flick, odorant molecules are advected between adjacent aesthetascs, while during the slower returnstroke, these odorants are trapped between the sensilla and molecular diffusion occurs over a sufficient time period to transport odorants to aesthetasc surfaces. During subsequent flicks, up to $97.6 \%$ of these odorants are replaced with new odorant molecules.
\end{abstract}


The concentration of molecules captured along aesthetasc surfaces was found to increase with increased gap spacing between aesthetascs, flick speed, and distance from the proximal end of the aesthetasc, but these changes in morphology and flicking kinematics reduce the animal's ability to take discrete samples of the odorant-laden fluid environment with each flick. Results suggest that antennule flicking allows discrete sampling of the time- and space-varying odorant signal, and high concentration odorant filaments can be distinguished from more diffuse, low concentration filaments through changes in both the timing and encounter rate of odorant molecules to aesthetasc surfaces.

\section{$2.1 \quad$ Introduction}

Chemosensing in aquatic crustaceans is a commonly observed phenomenon that enables these animals to locate food, habitat, and prey $[34,35,36]$ and engage in intraspecific communication [37]. Aquatic crustaceans have microscopic sensory organs, called aesthetascs, arrayed on their antennules that serve as organs of chemosensing. The antennules of decapod crustaceans such as lobsters, crabs and crayfish have two branches, called flagella, and the lateral flagellum of an antennule bears the arrays of olfactory aesthetasc sensilla. While a variety of chemosensory sensilla found on antennules, as well as on other appendages, are involved in various aspects of food-odor tracking [38], the aesthetascs alone appear to be involved in processing odorants from conspecifics [39, 37]. Although a great diversity of antennule morphologies are found across various aquatic species $[40][41,11,8]$, the mechanism behind odorant sensing in most of these organisms is a periodic flicking of their lateral flagellum against the flow. This results in advection and subsequent entrapment of odorant molecules adjacent to arrays of aesthetasc hairs $[13,11,29,28]$. Small-scale advection and molecular diffusion transports these molecules to the surface of the aesthetascs where odorants can be detected by the chemoreceptor neurons $[4,11]$.

Within aquatic environments, the distribution of odorants in both space and time is 
complex, and odorant plumes are often composed of filaments of chemicals at high concentrations adjacent to fluid with little or no odorant $[42,43,4,14]$. This filamentous odor structure varies with distance from the odorant source and depends on factors such as the scales of turbulence in the water flow, and the topography over which the fluid is moving $[44,8]$. Flicking in crustaceans has been shown to increase the fluid transport and the flux of odorants to aesthetasc surfaces $[11,13]$. This flicking is analogous to taking a 'sniff' of odor [45]. Although other studies have quantified the importance of antennule flicking on the leakiness of air or water surrounding aesthetascs, few have quantified how this behavior impacts the advective-diffusive transport of odorants to aesthetasc surfaces. Quantifying advective-diffusive transport of dissolved odorants in fluids is a much more complicated analysis and ultimately is more important in determining actual odorant detection. In addition, although trapping of fluid between adjacent aesthetascs has been studied in detail, discrete sampling of odorants has not.

The first step in the process of smelling is the interception of odorants in the surrounding water by olfactory organs. The relative importance of viscous to inertial forces in determining flow around a biological structure, such as an antennule or aesthetasc, is defined by the Reynolds number, $R e=\rho U L / \mu$, where $\rho$ is the density, $\mu$ is the dynamic viscosity, $U$ is the velocity and $L$ is the characteristic length scale of the biological structure (e.g. antennule or aesthetasc diameter). While the inertial forces for the flow at the length scale of an antennule during a flick cannot be ignored (such as turbulent mixing of odorant molecules in the antennule wake), the viscous forces at the length scale of an aesthetasc are much more dominant than inertial forces and the flow behavior is largely laminar. Reynolds number for sensillar hairs of various arthropods ranges from $10^{-4}$ to 10 [46]. Such a flow facilitates the development of a layer of slow moving fluid around the surface of the aesthetascs, called the boundary layer. The boundary layer is relatively thick for low Re, meaning that molecular diffusion is the primary mode of transport of odorant molecules adjacent to the surfaces of aesthetascs. Previous studies have shown that the time course of molecular diffusion of 
odorants to the chemoreceptors has a significant bearing on neural responses [18, 19, 20, 21], while others have argued that molecular diffusion does not play a limiting role in access of odorant molecules to chemoreceptors $[22,23,24]$. These hypotheses have been tested using calculations of odorant molecule flux to the aesthetasc surface based on assumptions regarding the near aesthetasc flow field $[25,26,27,28]$ or measurements of flow velocities surrounding aesthetascs utilizing particle-image velocimetry on dynamically scaled models $[13,29]$. However, due to the exceedingly small scales at which transport occurs, $\mathrm{O}(\mu \mathrm{m})$, direct observations or measurements of odorant transport to aesthetasc surfaces have proven difficult.

Despite our knowledge that flicking in crustaceans enhances response to odorants [47], the flow structure created by a flicking antennule and its impact on chemical transport to aesthetasc surfaces remains poorly understood. Therefore, numerical models are useful tools in understanding the impact of organism morphology and flow kinematics on chemosensing at these relatively small scales. The objective of this study is to determine the impact of antennule morphology and flicking kinematics on odorant molecule capture along aesthetasc surfaces. This study presents a numerical model of flow and odorant transport to the surfaces of aesthetascs along the lateral antennule of the freshwater crayfish, Procambarus clarkii. The aesthetascs on the antennules of P. clarkii usually occur in pairs on each lateral flagellar annulus, and this relatively simple geometry, combined with an understanding of the largescale flow around the antennular flagellum [48] enables us to calculate the time-course of odorant delivery to aesthetasc surfaces during a flick. The specific questions we address using this numerical model are:

1. What effect does the gap width between adjacent aesthetascs have on the concentration of molecules transported to the aesthetasc surface?

2. What effect does the velocity of the antennular flick have on the concentration of molecules transported to the aesthetasc surface? 
3. Do odorant filaments of different thickness affect the concentration of molecules transported to the aesthetasc surface?

4. Does the location along the aesthetasc surface impact the concentration of molecules transported to its surface?

\subsection{Methods}

The antennular flagella of P. clarkii contain arrays of aesthetascs that usually occur in pairs (but sometimes in an array of up to 4 aesthetascs), and are located distally on the ventral surface of the lateral antennular flagellum (figure 2.1(a) Antennular flagellum of Procambarus clarkii. Grid in background is $1 \mathrm{~cm}$ by $1 \mathrm{~cm}$. (b) Annuli of an antennule of P. clarkii. Aesthetasc sensilla (shown by black arrows) are arrayed ventrally on each annulus of the distal half of the flagellumfigure.caption.13 on page 11(a) Antennular flagellum of Procambarus clarkii. Grid in background is $1 \mathrm{~cm}$ by $1 \mathrm{~cm}$. (b) Annuli of an antennule of P. clarkii. Aesthetasc sensilla (shown by black arrows) are arrayed ventrally on each annulus of the distal half of the flagellumfigure.caption.13). These aesthetascs are small hair-like structures that contain about 170 olfactory receptor neurons (ORN) and have a thin cuticle that is permeable to odorant molecules [9]. The olfactory receptor neurons connect to the ipsilateral olfactory lobe of the brain and encode information provided by chemoreceptor proteins targeted to specific olfactory determinants [49]. Each aesthetasc is approximately $100 \mu \mathrm{m}$ in length, 15-20 $\mu \mathrm{m}$ in base diameter, and less than $10 \mu \mathrm{m}$ in tip diameter [9]. There is a $1.9 \mu \mathrm{m}$ thick cuticle that shows a notable change to transparency approximately three-fifths of the way from base to tip, where the majority of chemosensory cells are located [9]. The paired aesthetascs are spaced approximately one sensillum diameter apart. The flicking motion of the antennule is mediated by a single muscle, leading to a fast downstroke and a relatively slower return stroke [30]. Video recordings of adult P. clarkii indicate that antennule flicks occur over a $2 \mathrm{~mm}$ distance at average speeds of $1.8 \mathrm{~cm} \mathrm{~s}^{-1}$ and $1.1 \mathrm{~cm} \mathrm{~s}^{-1}$ for the downstroke 

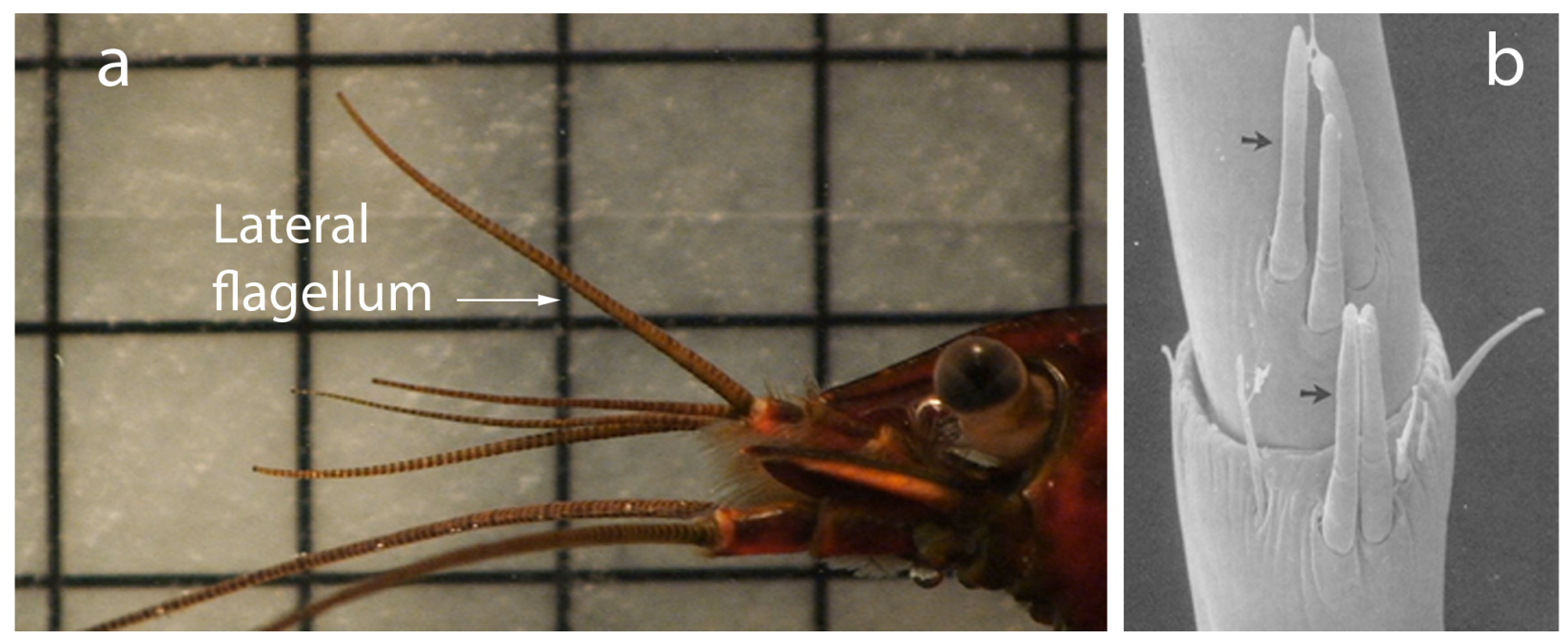

Figure 2.1: (a) Antennular flagellum of Procambarus clarkii. Grid in background is $1 \mathrm{~cm}$ by $1 \mathrm{~cm}$. (b) Annuli of an antennule of P. clarkii. Aesthetasc sensilla (shown by black arrows) are arrayed ventrally on each annulus of the distal half of the flagellum

and returnstroke, respectively. The average flicking rate of crayfish was measured to be 3.3 flicks $\mathrm{s}^{-1}$ but varies considerably depending upon environmental conditions [30], and flicks occur irregularly and infrequently when stimuli are lacking.

To determine how flicking and antennule morphology impact odorant transport to chemosensory sensilla, I constructed a two dimensional rectangular flow domain perpendicular to the antennule in which the fluid along with odorant enters and diffuses to the surface of the aesthetascs (figure 2.2Schematics of flow past the antennule and aesthetascsfigure.caption.14 on page 12Schematics of flow past the antennule and aesthetascsfigure.caption.14). The forward and return motions of the flicking antennule were numerically modeled by making the boundary condition a function of time which corresponds to the speed of a typical flick. The aesthetasc sensilla are oriented at 45 degrees to the antennules and we have used a circular shape to represent the cross section of the aesthetascs lying within the 2-D flow domain. The governing equations for this flow model are given by equations 2.1Methodsequation.2.2.12.3Methodsequation.2.2.3. 


$$
\begin{gathered}
\frac{\partial u}{\partial x}+\frac{\partial v}{\partial y}=0 \\
\frac{\partial u}{\partial t}+u \frac{\partial u}{\partial x}+v \frac{\partial u}{\partial y}=-\frac{1}{\rho} \frac{\partial p}{\partial x}+\nu\left(\frac{\partial^{2} u}{\partial x^{2}}+\frac{\partial^{2} u}{\partial y^{2}}\right) \\
\frac{\partial v}{\partial t}+u \frac{\partial v}{\partial x}+v \frac{\partial v}{\partial y}=-\frac{1}{\rho} \frac{\partial p}{\partial y}+\nu\left(\frac{\partial^{2} v}{\partial x^{2}}+\frac{\partial^{2} v}{\partial y^{2}}\right)
\end{gathered}
$$

where $\mathrm{u}$ and $\mathrm{v}$ are velocity components along $\mathrm{x}$ and $\mathrm{y}$ axes, $\rho$ is the density, $\nu$ is the kinematic viscosity and $p$ is the pressure. Equation 2.1Methodsequation.2.2.1 is the continuity equation for a two-dimensional incompressible flow and equations 2.2Methodsequation.2.2.2 and 2.3Methodsequation.2.2.3 represent momentum transport along $\mathrm{x}$ and $\mathrm{y}$ directions respectively. The velocity components obtained from these equations are used to calculate the odorant concentration from the convective-diffusive equation for scalar transport which is given by equation 2.4Methodsequation.2.2.4.

$$
\frac{\partial C}{\partial t}+u \frac{\partial C}{\partial x}+v \frac{\partial C}{\partial y}=D\left(\frac{\partial^{2} C}{\partial x^{2}}+\frac{\partial^{2} C}{\partial y^{2}}\right)
$$

where $D$ is the coefficient of diffusivity of the odorant in water. Typically odorants that attract crustaceans are composed of amino acids with a molecular diffusivity of $D=10^{-9} \mathrm{~m}^{2}$ $\mathrm{s}^{-1}[50]$.

The boundary conditions for the flow domain (figure 2.2Schematics of flow past the antennule and aesthetascsfigure.caption.14) are:

- Inlet boundary AB: The fluid is assumed to enter the inlet boundary with the inlet velocity being a parabolic function of time, to represent water motion relative to a flicking antennule. The antennule within the frame of reference is stationary and the boundary conditions therefore create the moving reference frame relative to the 


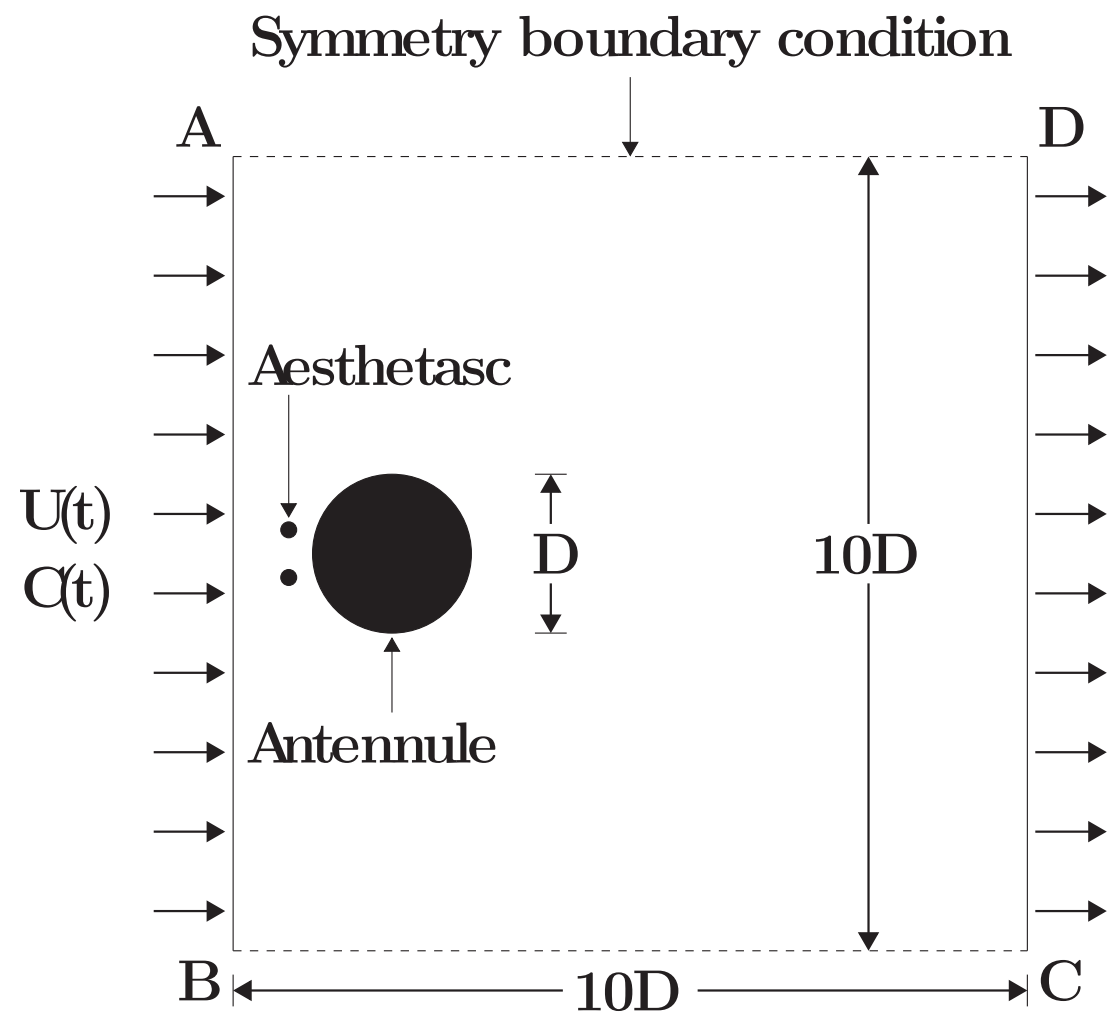

Figure 2.2: Schematics of flow past the antennule and aesthetascs

antennule. The water and odorant flow at the inlet are described by:

$$
\begin{gathered}
U(t)= \begin{cases}-4 U_{p f} \frac{t\left(t-t_{1}\right)}{t_{1}^{2}} & : 0<t<t_{1} \\
-4 U_{p r} \frac{\left(t-t_{1}\right)\left(t-t_{2}\right)}{t_{2}-t_{1}^{2}} & : t_{1}<t<t_{2}\end{cases} \\
C(t)= \begin{cases}-4 C_{\infty} \frac{t\left(t-t_{1}\right)}{t_{1}^{2}} & : 0<t<t_{1} \\
-4 C_{\infty} \frac{\left(t-t_{1}\right)\left(t-t_{2}\right)}{t_{2}-t_{1}^{2}} & : t_{1}<t<t_{2}\end{cases}
\end{gathered}
$$

where $U_{p f}\left(1.81 \mathrm{~cm} \mathrm{~s}^{-1}\right)$ and $U_{p r}\left(1.09 \mathrm{~cm} \mathrm{~s}^{-1}\right)$ are respectively the peak velocities for forward and return strokes of a flick, $C_{\infty}$ is the ambient concentration of odorant molecules, $t_{1}$ is the time duration of the downstroke and $t_{2}$ is the time duration of the entire forward and return motion. These boundary conditions are depicted in figure 2.3(a) Velocity and (b) odorant concentration at inlet boundary. Both the quantities are assumed to have a parabolic time dependent influx to represent the flicking motion 


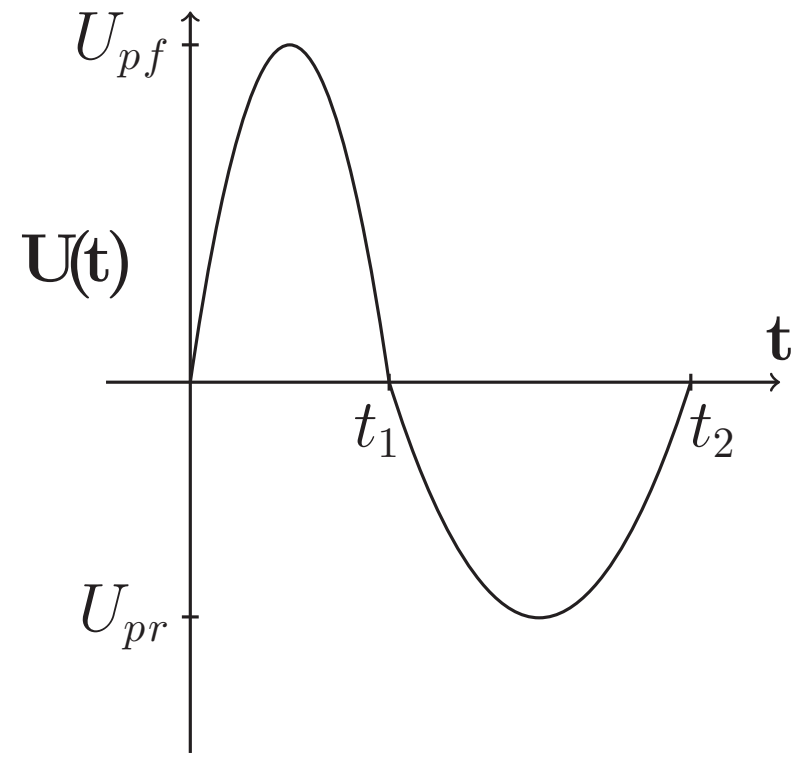

a

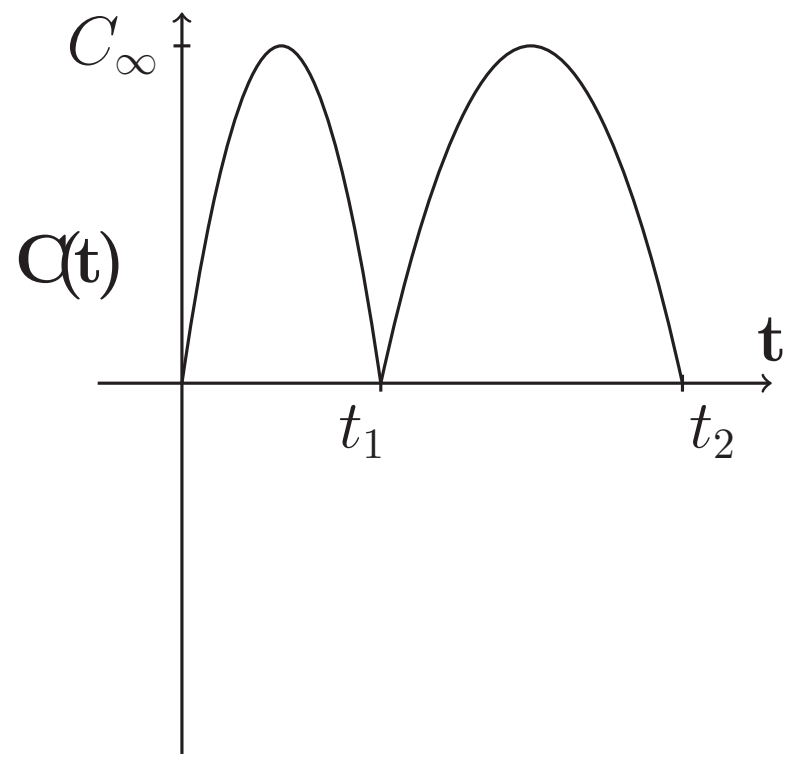

b

Figure 2.3: (a) Velocity and (b) odorant concentration at inlet boundary. Both the quantities are assumed to have a parabolic time dependent influx to represent the flicking motion of the antennule. $t_{1}=0.118 \mathrm{~s}$ represents the end of downstroke and $t_{2}=0.297 \mathrm{~s}$ represents the end of the entire forward-return sequence, as measured from video recordings of antennule flicking by adult $P$. clarkii

of the antennule. $t_{1}=0.118 \mathrm{~s}$ represents the end of downstroke and $t_{2}=0.297 \mathrm{~s}$ represents the end of the entire forward-return sequence, as measured from video recordings of antennule flicking by adult P. clarkiifigure.caption.15, where we have assumed a parabolic profile for both the forward and return strokes, which were approximated from motion analysis of crayfish flicking. The initial conditions assume that the crayfish begins the flick when there is an odorant-containing filament a distance $0.25 \mathrm{~mm}$ in front of the antennule, with a filament width thicker than a flick span, and a concentration of $C_{\infty}=10^{-8} \mathrm{~kg} \mathrm{~m}^{-3}$.

- Outlet boundary $C D$ : An 'open' boundary condition is used for velocity at the outlet boundary. Such a boundary condition allows the fluid to cross the boundary surface in either direction and allows for the model to conserve continuity (equation 2.1Methodsequation.2.2.1). The pressure is specified as $p_{\text {opening }}=p_{\text {specified }}$ where 
$p_{\text {specified }}=0$. This pressure is interpreted as relative total pressure for inflow and relative static pressure for outflow. For flow into the domain, the value of the concentration variable at the opening boundary is specified as $C_{\text {opening }}=0$. For flow out of the domain, no specified value is mandated.

- The top and bottom boundaries $A D$ and $B C$ : Symmetry boundary conditions are used for these boundaries, which impose constraints that mirror the flow on either side of it. The velocity component normal to the symmetry plane boundary is set to zero: $u_{y}=0$ and the concentration gradient normal to the boundary is also set to zero: $\partial C / \partial y=0$.

- Antennule and aesthetasc walls: The boundary condition at the walls of the antennule and aesthetascs is the no-slip condition for velocity $\left(u_{x}=u_{y}=0\right)$ and zero flux condition for concentration $\left(-D \nabla_{n} C=0\right)$, where $\nabla_{n} C$ is the gradient of concentration normal to the antennule/aesthetasc surface.

The flow domain was meshed into triangular and quadrilateral cells using the commercially available $\mathrm{CFX}^{\mathrm{TM}}$ Mesh tool. A denser mesh with inflated boundaries was created around the antennule and both aesthetascs to achieve a finer resolution in those regions. Figure 2.4Mesh grid cells around the aesthetascs and antennule. Cell size adjacent to the aesthetasc surface is $0.15 \mu \mathrm{m}$ and that around the antennule surface is $5 \mu$ mfigure.caption.16 shows a zoomed-in view of a high concentration of mesh grid cells close to the aesthetasc surface. Grid cells with characteristic size of $0.15 \mu \mathrm{m}$ are created around the aesthetasc surface while cells of size 5 $\mu \mathrm{m}$ are created around the antennule surface. A high resolution transient $\mathrm{CFX}^{\mathrm{TM}}$ solver is used to obtain fluid velocity and concentration values throughout the domain for the duration of a flick and return sequence. We have assumed that odorant molecules can be detected by neurons lining the aesthetasc surface if they are located at a distance of $0.15 \mu \mathrm{m}$ or less from the surface, i.e., within the grid adjacent to the aesthetasc surface. To be detected by neurons lining the aesthetasc surface, odorant molecules must first diffuse across the porous cuticle, whose thickness has been measured for P. clarkii as $1.9 \mu \mathrm{m}$ [9]. Assuming the diffusivity of 


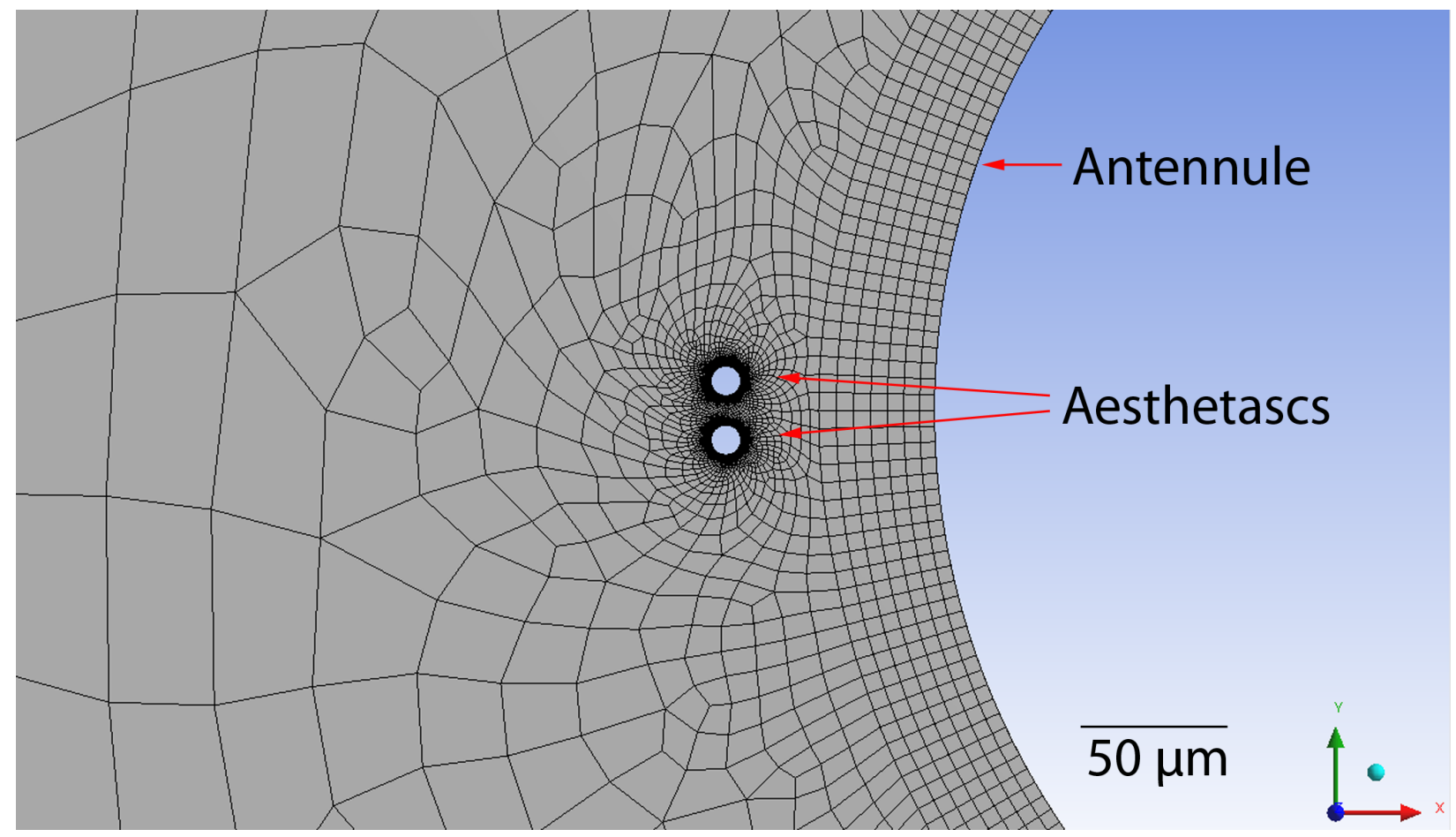

Figure 2.4: Mesh grid cells around the aesthetascs and antennule. Cell size adjacent to the aesthetasc surface is $0.15 \mu \mathrm{m}$ and that around the antennule surface is $5 \mu \mathrm{m}$

odorant molecules through the cuticle is similar to that of odorant molecules in water, the timescale for diffusion can be computed as $t=x_{r m s}^{2} / 2 D=2.1 \mathrm{~ms}$ [51], where $x_{r m s}$ is the root mean squared distance of odorant diffusion. Hence the assumption is that the odorant molecules present in the grid cell adjacent to the aesthetasc surface will diffuse quickly across the cuticle to chemosensory neurons and can serve as a proxy for odorant concentration that is detected. The average concentration of odorants on the aesthetasc surface over the duration of a flick, $C_{\text {mean }}$, is calculated from the average of grid cells located adjacent to the aesthetasc surface. In addition, four points, evenly distributed around each of the aesthetascs, are examined to provide insight into the distribution of odorant concentrations at different locations along the aesthetasc surface. 


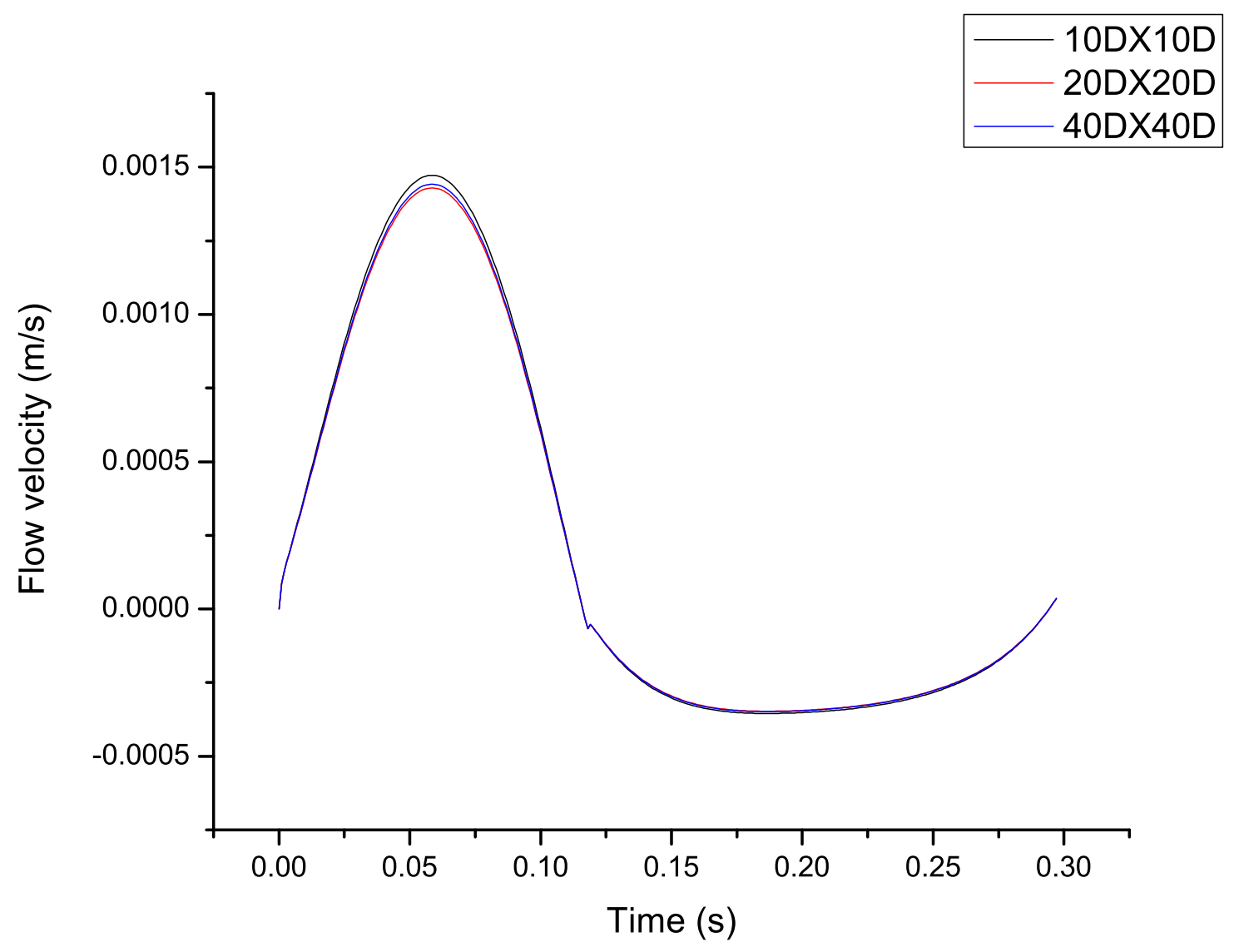

Figure 2.5: Flow velocity between the aesthetascs over the course of a flick for different sizes of the flow domain.

\subsubsection{Model independence}

To ascertain the independence of the model from CFD domain size, the flow velocity at midpoint between the two aesthetascs is compared at three different flow domain sizes of 10Dx10D, 20Dx20D and 40Dx40D ( $\mathrm{D}=$ antennule diameter). No significant change in the flow temporal profile is observed at these sizes (Figure 2.5Flow velocity between the aesthetascs over the course of a flick for different sizes of the flow domain.figure.caption.17), leading to the conclusion that flow dynamics close to the aesthetascs is largely unaffected by far-field conditions even for a domain size as small as 10D. This suggests that the presence of a much larger antennule behind the aesthetascs effectively shields the flow regime between 
them. Hence a flow domain of 10Dx10D was used for the CFD model, providing an accurate and economical representation of the flow dynamics of interest in the model.

\subsection{Results}

Concentration contours (normalized by $C_{\infty}$ ) at the end of downstroke and return stroke are shown in figure 2.6Concentration contours (normalized by $C_{\infty}$ ) at the end of (a) downstroke and (b) returnstroke, and zoomed-in images of concentration surrounding the aesthetascs at the end of the (c) downstroke and (d) returnstroke. A region of trapped odorant molecules between the aesthetascs can be seen at the end of return stroke.figure.caption.18 for both the entire domain (figure 2.6Concentration contours (normalized by $C_{\infty}$ ) at the end of (a) downstroke and (b) returnstroke, and zoomed-in images of concentration surrounding the aesthetascs at the end of the (c) downstroke and (d) returnstroke. A region of trapped odorant molecules between the aesthetascs can be seen at the end of return stroke.figure.caption.18a and figure 2.6Concentration contours (normalized by $C_{\infty}$ ) at the end of (a) downstroke and (b) returnstroke, and zoomed-in images of concentration surrounding the aesthetascs at the end of the (c) downstroke and (d) returnstroke. A region of trapped odorant molecules between the aesthetascs can be seen at the end of return stroke.figure.caption.18b) and for the zoomed-in domain surrounding the aesthetascs (figure 2.6Concentration contours (normalized by $C_{\infty}$ ) at the end of (a) downstroke and (b) returnstroke, and zoomed-in images of concentration surrounding the aesthetascs at the end of the (c) downstroke and (d) returnstroke. A region of trapped odorant molecules between the aesthetascs can be seen at the end of return stroke.figure.caption.18c and figure 2.6Concentration contours (normalized by $C_{\infty}$ ) at the end of (a) downstroke and (b) returnstroke, and zoomed-in images of concentration surrounding the aesthetascs at the end of the (c) downstroke and (d) returnstroke. A region of trapped odorant molecules between the aesthetascs can be seen at the end of return stroke.figure.caption.18d). The concentration contour at the end of the 
flick (figure 2.6Concentration contours (normalized by $C_{\infty}$ ) at the end of (a) downstroke and (b) returnstroke, and zoomed-in images of concentration surrounding the aesthetascs at the end of the (c) downstroke and (d) returnstroke. A region of trapped odorant molecules between the aesthetascs can be seen at the end of return stroke.figure.caption.18d) shows a significant amount of odorant surrounding the aesthetascs after the flick has finished and hence provides evidence for entrapment of odorant molecules between the aesthetasc pair. Locations on the aesthetasc surface corresponding to $\theta=180^{\circ}$ (location on the aesthetasc that faces the ambient flow) and $\theta=270^{\circ}$ (location on the aesthetasc that faces the paired aesthetasc) receive the highest concentration of odorants, followed by locations at $\theta=90^{\circ}$ and $\theta=0^{\circ}$, respectively (figure 2.7 Normalized odorant concentration at four different locations on the surface of an aesthetasc during a downstroke/returnstroke flick sequence. For this aesthetasc geometry, $\theta=180^{\circ}$ represents the location on the aesthetasc that faces the ambient flow, while $\theta=270^{\circ}$ represents the location on the aesthetasc that faces the other aesthetasc.figure.caption.19). Overall, the rate of change of odorant concentration with time is greatest at the front edge of the aesthetasc and along the surface facing away from the paired aesthetasc (figure 2.8Rate of normalized concentration change at four different locations on the surface of an aesthetasc during a downstroke/returnstroke flick sequence. For this aesthetasc geometry, $\theta=180^{\circ}$ represents the location on the aesthetasc that faces the ambient flow, while $\theta=270^{\circ}$ represents the location on the aesthetasc that faces the other aesthetasc.figure.caption.20).

The flushing of previously captured odorant and its replacement with new odorant molecules during successive flicks is vital to allow for discrete sampling of odorant distributions within a crustacean's surroundings. To test the relative magnitude of the exchange of odorant molecules to aesthetasc surfaces between successive flicks, a set of simulations was conducted where the initial condition for odorant molecules concentrations within the flow domain was taken to be the concentration of trapped molecules between the aesthetascs resulting from the previous flick (as shown in figure 2.6Concentration contours (normalized by $C_{\infty}$ ) at the end of 

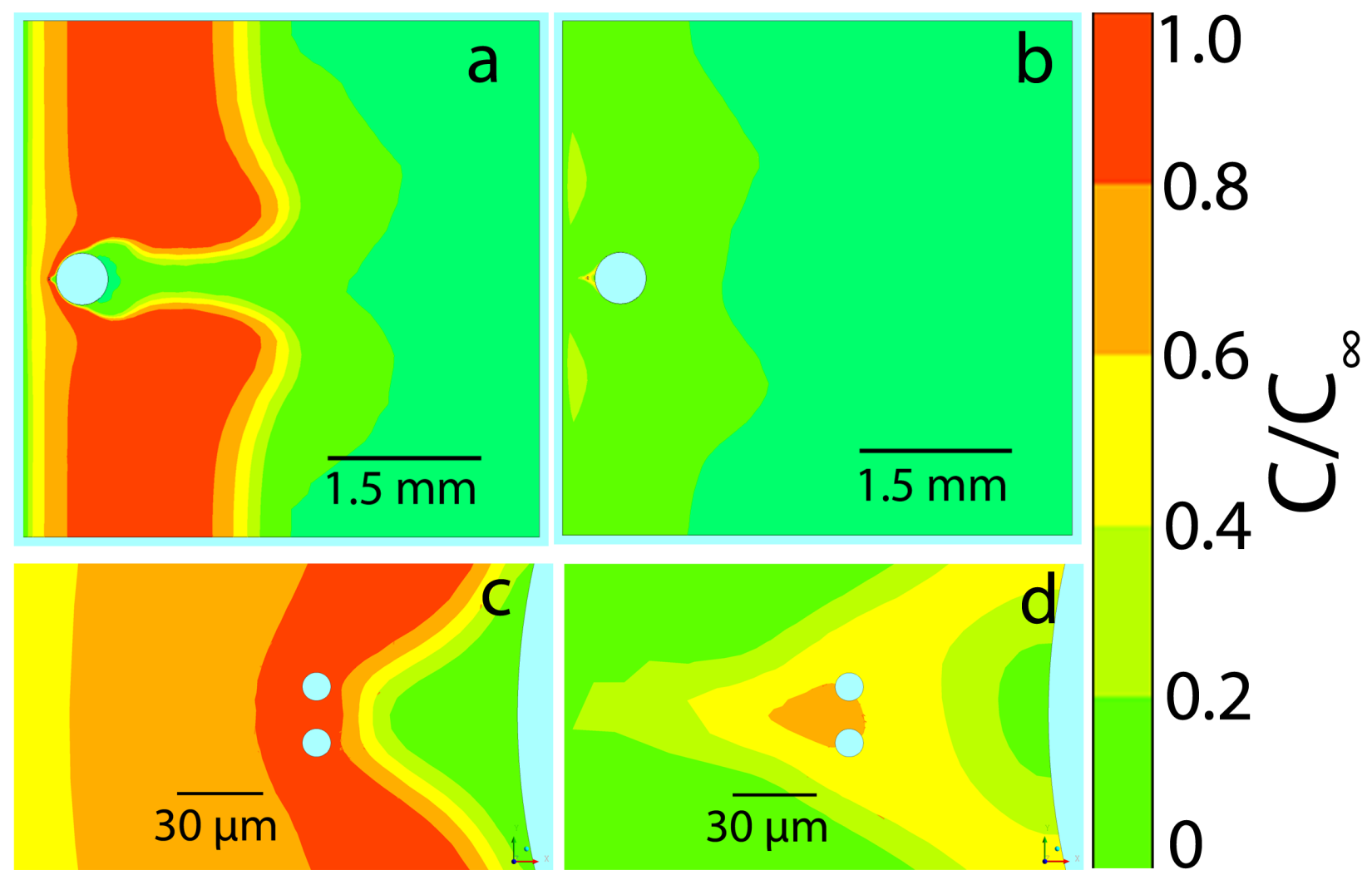

Figure 2.6: Concentration contours (normalized by $C_{\infty}$ ) at the end of (a) downstroke and (b) returnstroke, and zoomed-in images of concentration surrounding the aesthetascs at the end of the (c) downstroke and (d) returnstroke. A region of trapped odorant molecules between the aesthetascs can be seen at the end of return stroke.

(a) downstroke and (b) returnstroke, and zoomed-in images of concentration surrounding the aesthetascs at the end of the (c) downstroke and (d) returnstroke. A region of trapped odorant molecules between the aesthetascs can be seen at the end of return stroke.figure.caption.18d). Two types of inlet boundary conditions were prescribed: (1) the antennule flicking into an odorant filament of concentration $C_{\infty}$ or $(2)$ the antennule flicking into fresh water $(\mathrm{C}=0$ $\mathrm{kg} \mathrm{m}^{-3}$ ). Figure 2.9Concentration at aesthetasc surface for a second flick following an initial flick. Components of total odorant concentration are new odorant molecules acquired in the second flick and the old molecules remaining from the initial flick.figure.caption.21 shows the concentration levels for both cases. The total concentration levels observed in figure 2.9Concentration at aesthetasc surface for a second flick following an initial flick. Components of total odorant concentration are new odorant molecules acquired in the second flick and the 


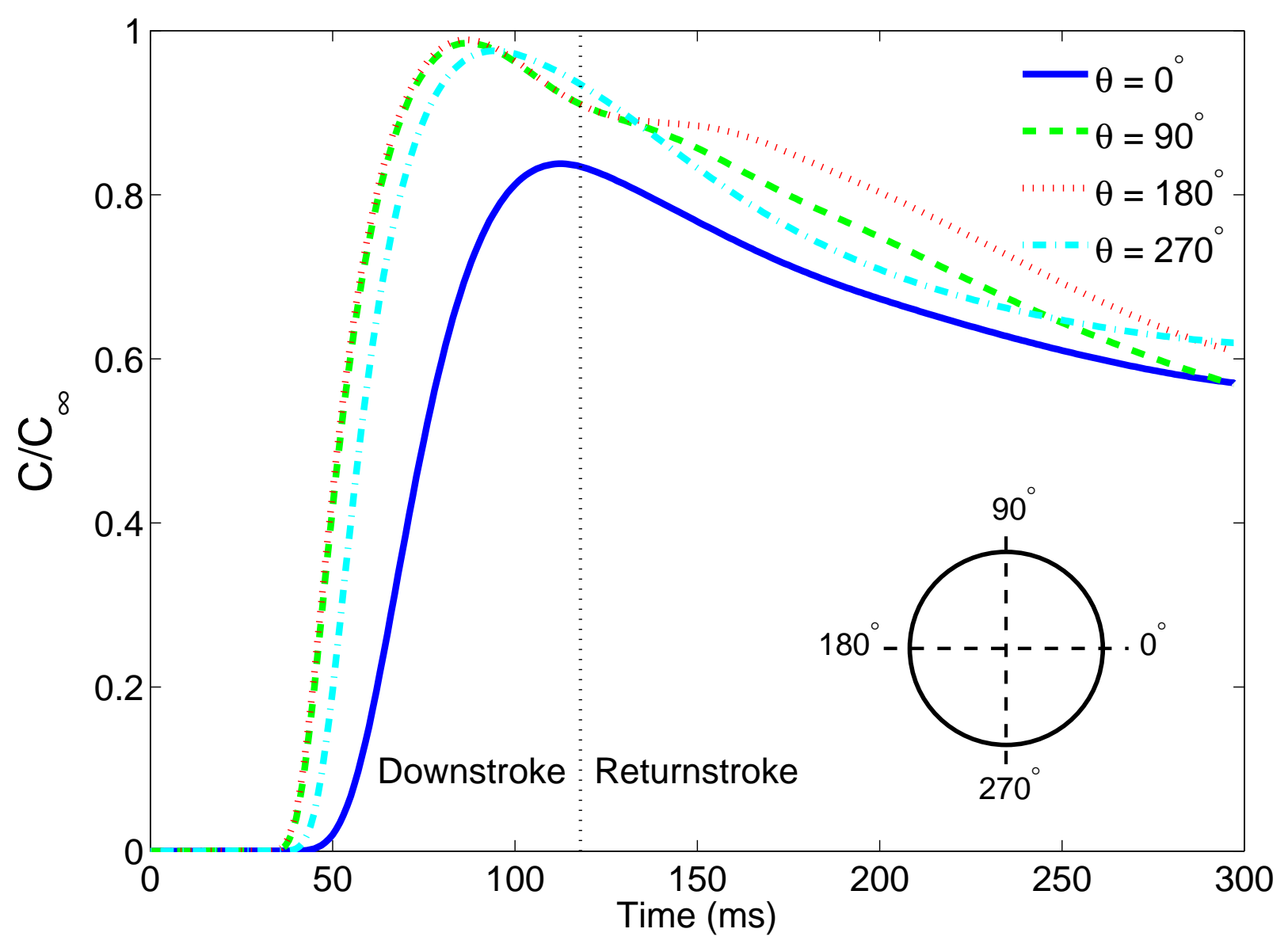

Figure 2.7: Normalized odorant concentration at four different locations on the surface of an aesthetasc during a downstroke/returnstroke flick sequence. For this aesthetasc geometry, $\theta=180^{\circ}$ represents the location on the aesthetasc that faces the ambient flow, while $\theta=270^{\circ}$ represents the location on the aesthetasc that faces the other aesthetasc.

old molecules remaining from the initial flick.figure.caption.21 gives the combined value of both new and old odorant concentrations while the line labeled 'old' gives the concentration of old molecules only. The subtraction of the two lines gives the concentration of new molecules captured during a successive flick. The line labeled 'new' indicates that $97.6 \%$ of odorants along the surface of the aesthetasc have been replaced by new molecules by the end of the downward flick. During the return stroke, flow reversal leads to some mixing of new molecules with residual old molecules from the previous flick in the wake of the antennule. This causes an increase in the proportion of old molecules sampled, but the new molecules still constitute $78.1 \%$ of total molecules sampled on the aesthetasc surface at the end of the return stroke. 


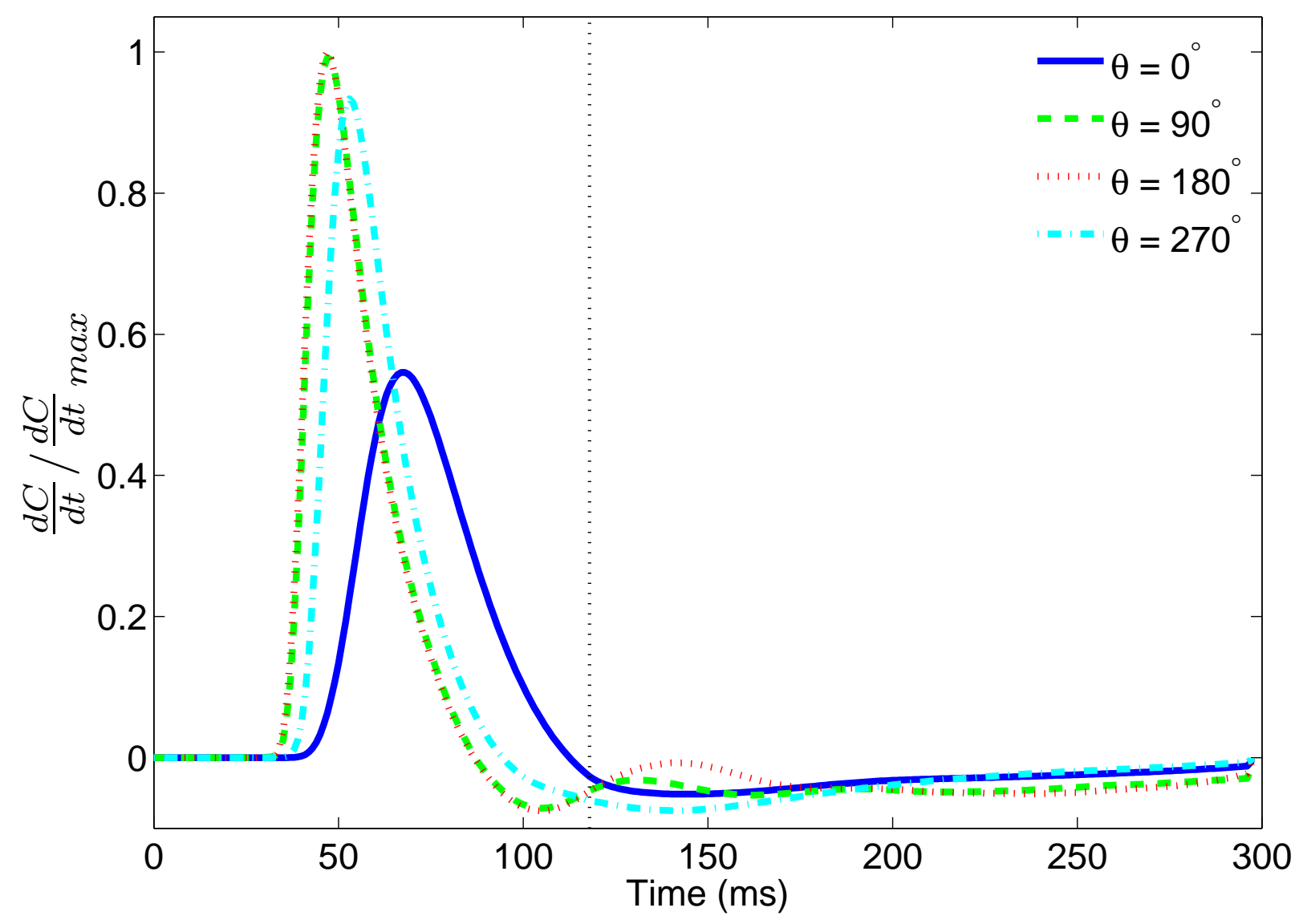

Figure 2.8: Rate of normalized concentration change at four different locations on the surface of an aesthetasc during a downstroke/returnstroke flick sequence. For this aesthetasc geometry, $\theta=180^{\circ}$ represents the location on the aesthetasc that faces the ambient flow, while $\theta=270^{\circ}$ represents the location on the aesthetasc that faces the other aesthetasc.

These results provide evidence that: (1) the old molecules are effectively flushed out and (2) new odorant molecules are captured along the aesthetasc surface during a subsequent flick.

To determine the impact of different odorant filament thicknesses on molecule capture, we examined the case when the organism encounters filaments of varying widths, either at the start or near the end of its downstroke. An antennule flicking into a thin odorant filament spends a part of its flick time outside the odorant-laden region. A schematic representing such a situation is shown in figure 2.10Illustration of an antennule at various stages of a downstroke as it passes through an odorant filament. The shaded region shows the area that constitutes the odorant. The antennule starts (a) in front of the filament, (b) gets completely 


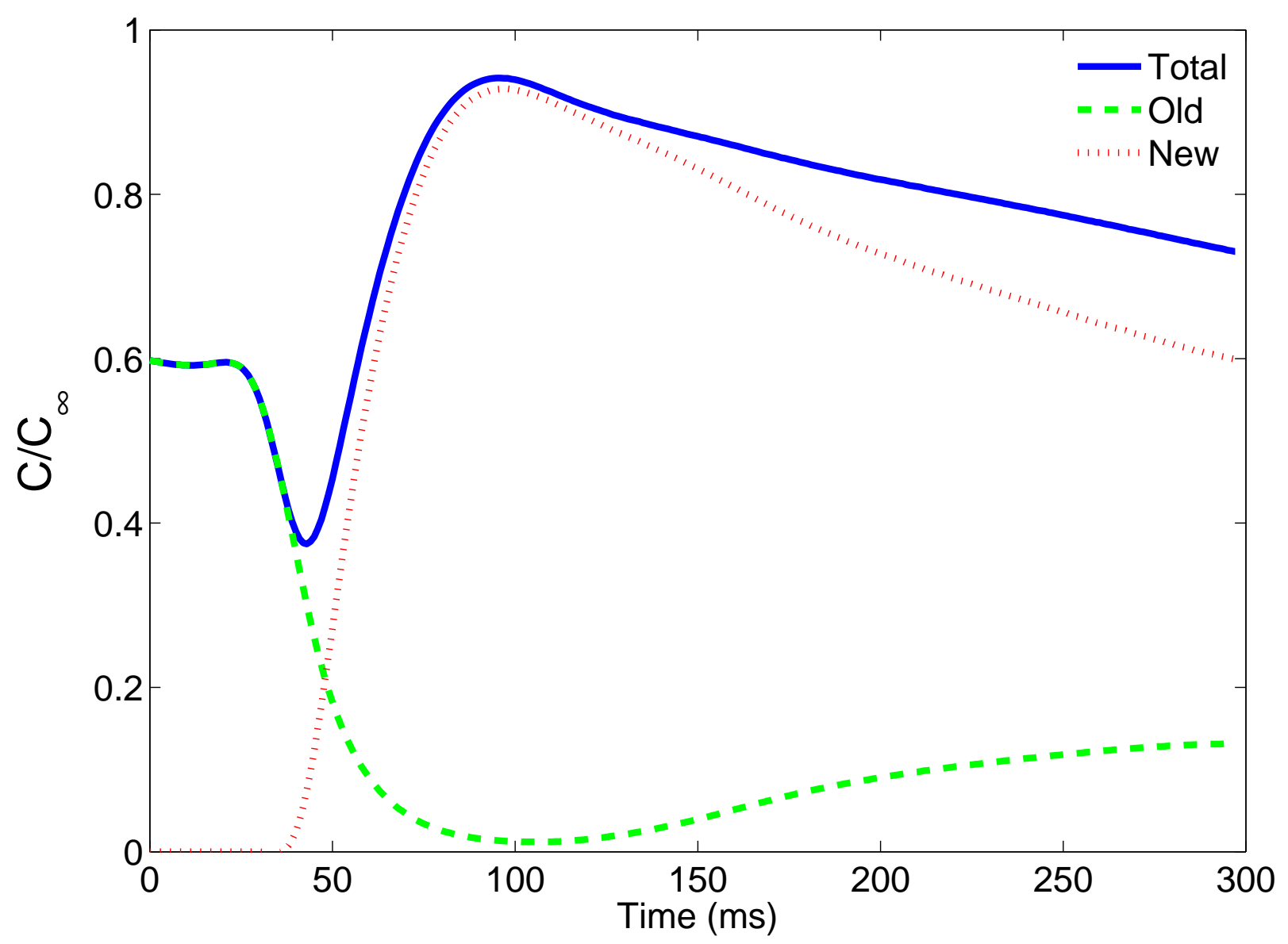

Figure 2.9: Concentration at aesthetasc surface for a second flick following an initial flick. Components of total odorant concentration are new odorant molecules acquired in the second flick and the old molecules remaining from the initial flick.

immersed in the filament and (c) traverses through the odorant filament towards the end of the downstroke. This sequence is repeated in reverse order as the antennule comes back through the filament during the return strokefigure.caption.22. The boundary condition for a filament placed at the beginning of a flick is given by equation 2.7Resultsequation.2.3.7.

$$
C(t)=\left\{\begin{array}{lll}
-4 C_{\infty} \frac{t\left(t-t_{1}\right)}{t_{1}^{2}} & : & 0<t<T_{1} \\
0 & : & T_{1}<t<T_{2} \\
-4 C_{\infty} \frac{\left(t-t_{1}\right)\left(t-t_{2}\right)}{t_{2}-t_{1}^{2}} & : & T_{2}<t<t_{2}
\end{array}\right.
$$


where $T_{1}$ is the time when the antennule exits the odorant filament during the downstroke and $T_{2}$ is the time when it reenters the odorant filament region during the return stroke. Boundary conditions for velocity and concentration are shown in figure 2.11Boundary conditions for (a) velocity and (b) odorant concentration for an odorant filament placed at the start of a downstroke. The solid lines and the shaded region show the portion of the flick that is spent by the antennule inside the filament. Odorant filaments grow thicker as both $T_{1}$ and $T_{2}$ move closer to $t_{1}$ figure.caption.23 for an odorant filament encountered at the beginning of the downstroke. A similar boundary condition is employed for the filaments encountered at the end of a downstroke. The filament sizes tested were varied by the values of $T_{1}$ and $T_{2}$ given in table 2.1 Values of $T_{1}$ and $T_{2}$ (the time instants that signify the temporal bounds of an odorant filament as shown in Fig. 10) for filaments encountered at the beginning of a flick.table.caption.24 and table 2.2Values of $T_{1}$ and $T_{2}$ (the time instants that signify the temporal bounds of an odorant filament as shown in Fig. 10) for filaments encountered at the end of a flick.table.caption.25 for filaments encountered at start and end of a downstroke respectively. For both encounters with filaments at the start (figure 2.12Variation of normalized mean concentration of odorant molecules around the aesthetasc surface with (a) odorant filaments of various width encountered during the beginning of the downstroke motion of a flick, (b) odor filaments of various width encountered during the end of the downstroke, (c) aesthetasc spacing, (d) flick speed, and (e) position of sensing at different distal distances along the length of an aesthetasc. Boxplots indicate the variation of odorant concentration transported to the surface of the aesthetasc. Horizontal line within the box indicates the median, while the lower and upper edges of the box represent the 25th and 75th percentiles, respectively. Vertical lines extending from the box indicate the minimum and maximum measured values.figure.caption.26A) and end (figure 2.12Variation of normalized mean concentration of odorant molecules around the aesthetasc surface with (a) odorant filaments of various width encountered during the beginning of the downstroke motion of a flick, (b) odor filaments of various width encountered during the end of the 


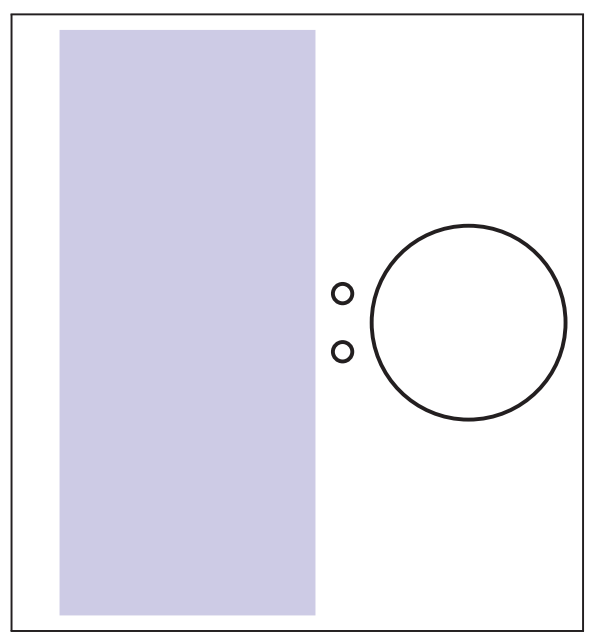

a

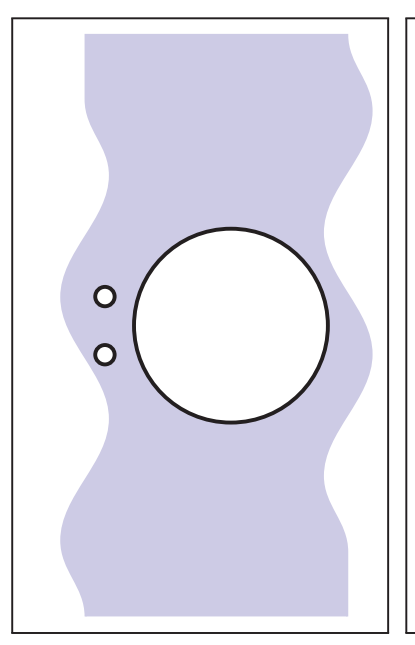

b
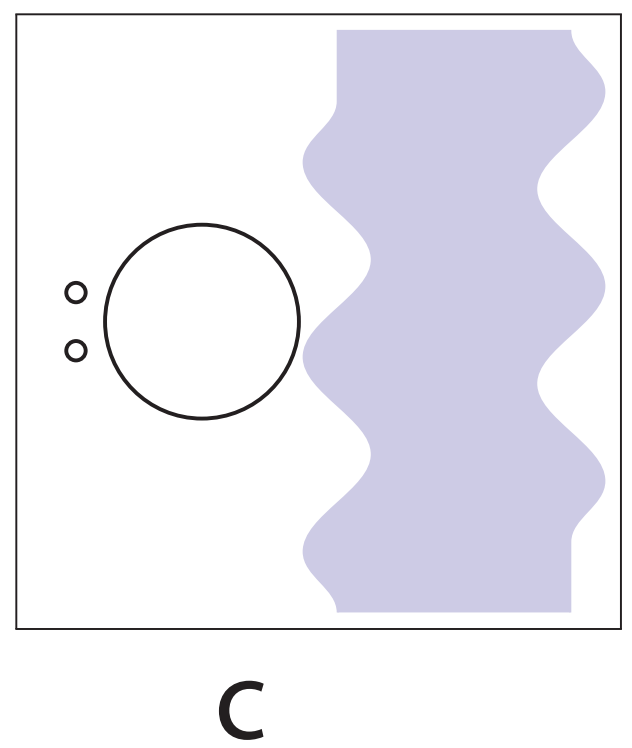

Figure 2.10: Illustration of an antennule at various stages of a downstroke as it passes through an odorant filament. The shaded region shows the area that constitutes the odorant. The antennule starts (a) in front of the filament, (b) gets completely immersed in the filament and (c) traverses through the odorant filament towards the end of the downstroke. This sequence is repeated in reverse order as the antennule comes back through the filament during the return stroke

Table 2.1: Values of $T_{1}$ and $T_{2}$ (the time instants that signify the temporal bounds of an odorant filament as shown in Fig. 10) for filaments encountered at the beginning of a flick.

\begin{tabular}{llllllll}
\hline$T_{1}(\mathrm{~s})$ & $0.125 t_{1}$ & $0.25 t_{1}$ & $0.375 t_{1}$ & $0.5 t_{1}$ & $0.625 t_{1}$ & $0.75 t_{1}$ & $0.875 t_{1}$ \\
$T_{2}(\mathrm{~s})$ & 0.2752 & 0.2536 & 0.2320 & 0.2106 & 0.1896 & 0.1695 & 0.1518 \\
\hline
\end{tabular}

downstroke, (c) aesthetasc spacing, (d) flick speed, and (e) position of sensing at different distal distances along the length of an aesthetasc. Boxplots indicate the variation of odorant concentration transported to the surface of the aesthetasc. Horizontal line within the box indicates the median, while the lower and upper edges of the box represent the 25th and 75th percentiles, respectively. Vertical lines extending from the box indicate the minimum and maximum measured values.figure.caption.26B) of a flick, thinner filaments correspond to lower concentrations sampled.

Variations in mean odorant concentration transported to the surfaces of aesthetascs due to changes in antennule morphology and flicking kinematics are shown in figure 2.12Variation of normalized mean concentration of odorant molecules around the aesthetasc surface with 


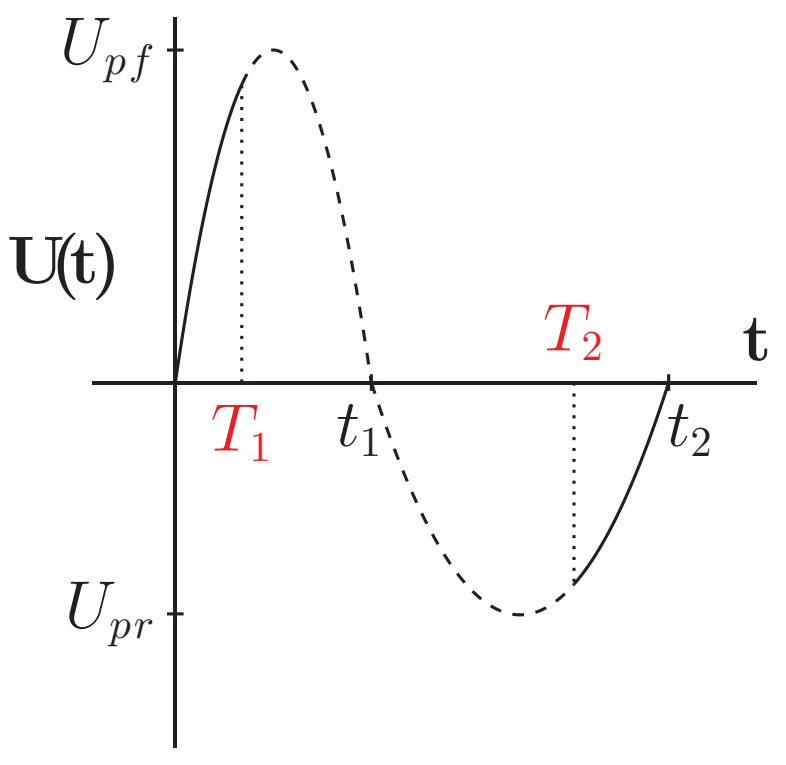

a

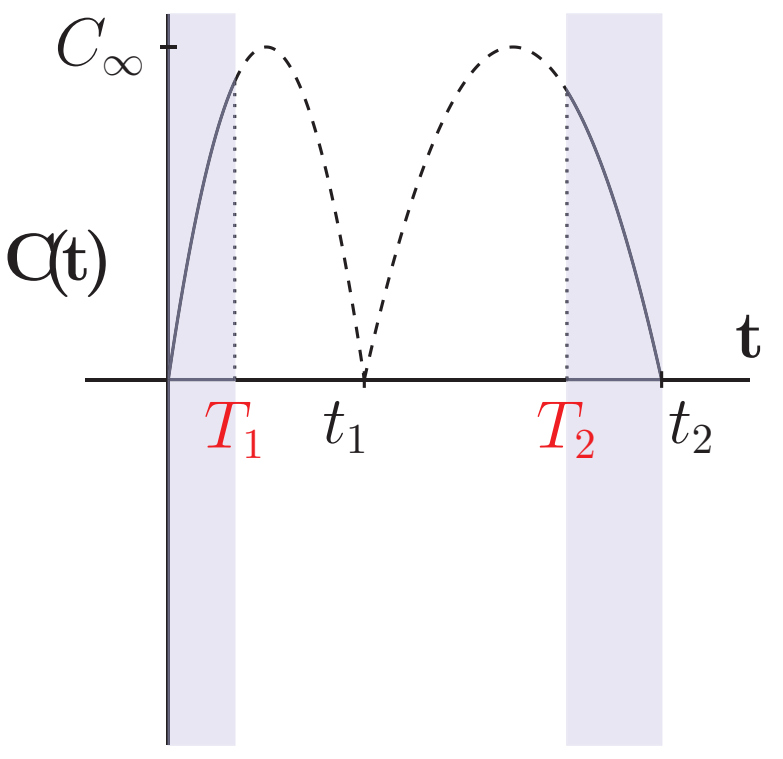

b

Figure 2.11: Boundary conditions for (a) velocity and (b) odorant concentration for an odorant filament placed at the start of a downstroke. The solid lines and the shaded region show the portion of the flick that is spent by the antennule inside the filament. Odorant filaments grow thicker as both $T_{1}$ and $T_{2}$ move closer to $t_{1}$

Table 2.2: Values of $T_{1}$ and $T_{2}$ (the time instants that signify the temporal bounds of an odorant filament as shown in Fig. 10) for filaments encountered at the end of a flick.

\begin{tabular}{llllllll}
\hline$T_{1}(\mathrm{~s})$ & $0.125 t_{1}$ & $0.25 t_{1}$ & $0.375 t_{1}$ & $0.5 t_{1}$ & $0.625 t_{1}$ & $0.75 t_{1}$ & $0.875 t_{1}$ \\
$T_{2}(\mathrm{~s})$ & 0.2632 & 0.2455 & 0.2253 & 0.2044 & 0.1830 & 0.1614 & 0.1396 \\
\hline
\end{tabular}

(a) odorant filaments of various width encountered during the beginning of the downstroke motion of a flick, (b) odor filaments of various width encountered during the end of the downstroke, (c) aesthetasc spacing, (d) flick speed, and (e) position of sensing at different distal distances along the length of an aesthetasc. Boxplots indicate the variation of odorant concentration transported to the surface of the aesthetasc. Horizontal line within the box indicates the median, while the lower and upper edges of the box represent the 25th and 75th percentiles, respectively. Vertical lines extending from the box indicate the minimum and maximum measured values.figure.caption.26. The gap width between adjacent aesthetascs was modified between 0.1 and 5 aesthetasc diameters (figure 2.12Variation of normalized 

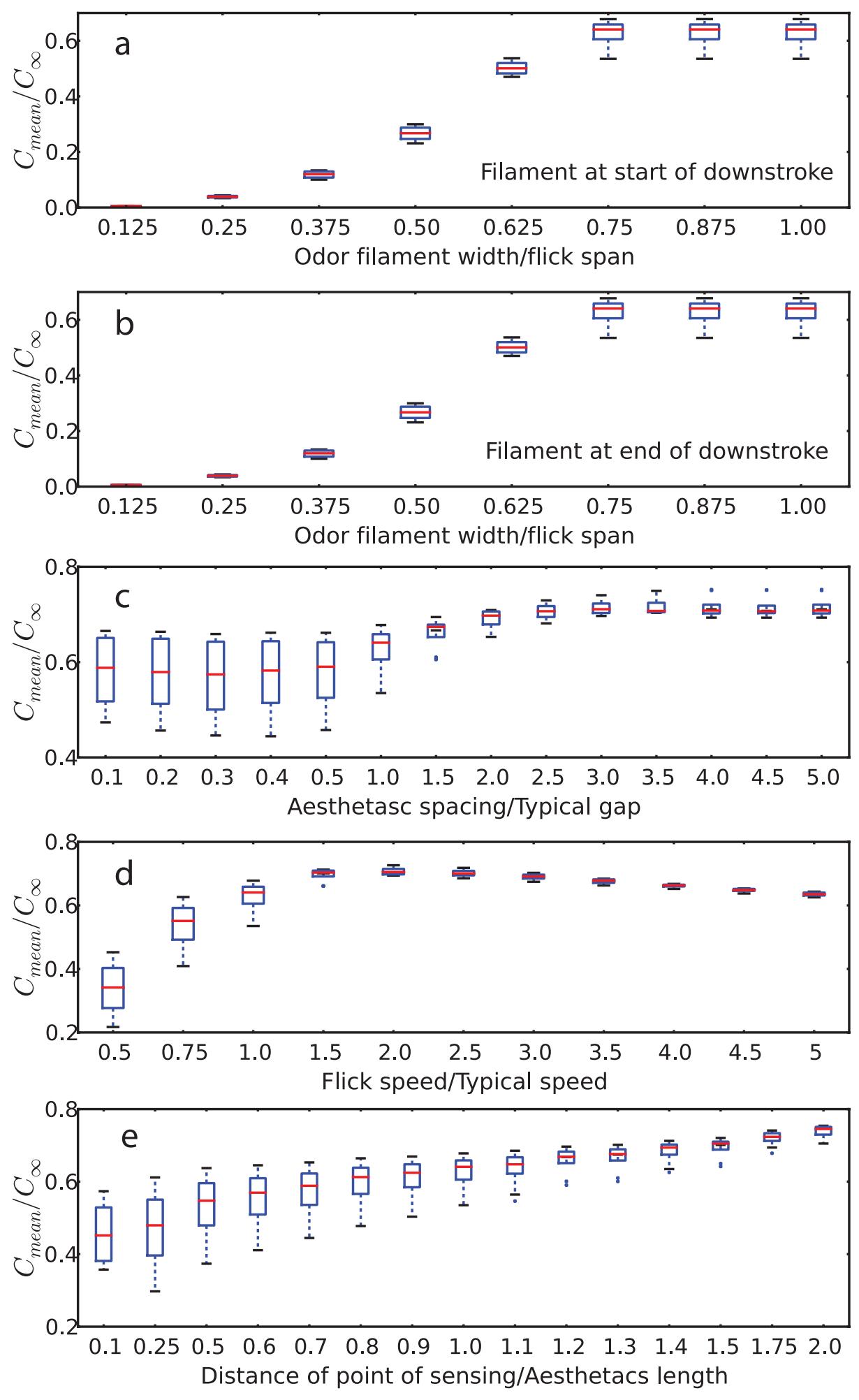

Figure 2.12: Variation of normalized mean concentration of odorant molecules around the aesthetasc surface with (a) odorant filaments of various width encountered during the beginning of the downstroke motion of a flick, (b) odor filaments of various width encountered during the end of the downstroke, (c) aesthetasc spacing, (d) flick speed, and (e) position of sensing at different distal distances along the length of an aesthetasc. Boxplots indicate the variation of odorant concentration transported to the surface of the aesthetasc. Horizontal line within the box indicates the median, while the lower and upper edges of the box represent the 25 th and 75 th percentiles, respectively. Vertical lines extending from the box indicate the minimum and maximum measured values. 
mean concentration of odorant molecules around the aesthetasc surface with (a) odorant filaments of various width encountered during the beginning of the downstroke motion of a flick, (b) odor filaments of various width encountered during the end of the downstroke, (c) aesthetasc spacing, (d) flick speed, and (e) position of sensing at different distal distances along the length of an aesthetasc. Boxplots indicate the variation of odorant concentration transported to the surface of the aesthetasc. Horizontal line within the box indicates the median, while the lower and upper edges of the box represent the 25 th and 75 th percentiles, respectively. Vertical lines extending from the box indicate the minimum and maximum measured values.figure.caption.26c). Molecule capture rises slightly with an increase in gap width and creates a significantly more uniform distribution of molecules around the aesthetasc surface. Increasing the antennule flicking speed enhances odorant molecule capture up to approximately twice the typical flick speed, above which odorant molecule capture declines for faster flick speeds (figure 2.12Variation of normalized mean concentration of odorant molecules around the aesthetasc surface with (a) odorant filaments of various width encountered during the beginning of the downstroke motion of a flick, (b) odor filaments of various width encountered during the end of the downstroke, (c) aesthetasc spacing, (d) flick speed, and (e) position of sensing at different distal distances along the length of an aesthetasc. Boxplots indicate the variation of odorant concentration transported to the surface of the aesthetasc. Horizontal line within the box indicates the median, while the lower and upper edges of the box represent the 25th and 75 th percentiles, respectively. Vertical lines extending from the box indicate the minimum and maximum measured values.figure.caption.26d). Figure 2.12Variation of normalized mean concentration of odorant molecules around the aesthetasc surface with (a) odorant filaments of various width encountered during the beginning of the downstroke motion of a flick, (b) odor filaments of various width encountered during the end of the downstroke, (c) aesthetasc spacing, (d) flick speed, and (e) position of sensing at different distal distances along the length of an aesthetasc. Boxplots indicate the variation of odorant concentration transported to the surface of the aesthetasc. Horizontal line within 
the box indicates the median, while the lower and upper edges of the box represent the 25th and 75th percentiles, respectively. Vertical lines extending from the box indicate the minimum and maximum measured values.figure.caption.26e shows the change in mean concentration sampled along the length of the aesthetasc from the proximal end to distal tip. The data revealed a clear trend of increased mean concentration sampled from the proximal end to the basal tip of the aesthetasc. Since only the distal two-fifths of the aesthetasc sensillum is believed to be permeable to odorant molecules, this region is able to sample greater relative concentrations. In addition, we modeled the odorant transport assuming that aesthetascs were longer than their typical length of $100 \mu \mathrm{m}$. The sampled odorant concentration increases with longer aesthetascs, even up to twice their normal length. The flushing efficiency, quantified as the fraction of old molecules displaced by new molecules during subsequent flicks, was determined as a function of aesthetasc spacing, flick speed, and position of sensing along the length of an aesthetasc (figure 2.13Flushing efficiency, quantified as the fraction of old molecules displaced by new molecules during subsequent flicks, plotted in relation to (a) aesthetasc spacing, (b) flick speed, and (c) position of sensing at different distal distances along the length of an aesthetasc. Boxplots are described in figure 2.12Variation of normalized mean concentration of odorant molecules around the aesthetasc surface with (a) odorant filaments of various width encountered during the beginning of the downstroke motion of a flick, (b) odor filaments of various width encountered during the end of the downstroke, (c) aesthetasc spacing, (d) flick speed, and (e) position of sensing at different distal distances along the length of an aesthetasc. Boxplots indicate the variation of odorant concentration transported to the surface of the aesthetasc. Horizontal line within the box indicates the median, while the lower and upper edges of the box represent the 25th and 75th percentiles, respectively. Vertical lines extending from the box indicate the minimum and maximum measured values.figure.caption.26. Smaller boxplots indicate more uniform concentrations sampled along the circumference of the aesthetasc.figure.caption.27). This flushing efficiency, signifies the effectiveness of a subsequent, second flick to replace 
previously captured odorants along the surface of the aesthetasc. Flushing efficiency increases with both aesthetasc spacing (figure 2.13Flushing efficiency, quantified as the fraction of old molecules displaced by new molecules during subsequent flicks, plotted in relation to (a) aesthetasc spacing, (b) flick speed, and (c) position of sensing at different distal distances along the length of an aesthetasc. Boxplots are described in figure 2.12Variation of normalized mean concentration of odorant molecules around the aesthetasc surface with (a) odorant filaments of various width encountered during the beginning of the downstroke motion of a flick, (b) odor filaments of various width encountered during the end of the downstroke, (c) aesthetasc spacing, (d) flick speed, and (e) position of sensing at different distal distances along the length of an aesthetasc. Boxplots indicate the variation of odorant concentration transported to the surface of the aesthetasc. Horizontal line within the box indicates the median, while the lower and upper edges of the box represent the 25 th and 75 th percentiles, respectively. Vertical lines extending from the box indicate the minimum and maximum measured values.figure.caption.26. Smaller boxplots indicate more uniform concentrations sampled along the circumference of the aesthetasc.figure.caption.27a) and flick speed (figure 2.13Flushing efficiency, quantified as the fraction of old molecules displaced by new molecules during subsequent flicks, plotted in relation to (a) aesthetasc spacing, (b) flick speed, and (c) position of sensing at different distal distances along the length of an aesthetasc. Boxplots are described in figure 2.12Variation of normalized mean concentration of odorant molecules around the aesthetasc surface with (a) odorant filaments of various width encountered during the beginning of the downstroke motion of a flick, (b) odor filaments of various width encountered during the end of the downstroke, (c) aesthetasc spacing, (d) flick speed, and (e) position of sensing at different distal distances along the length of an aesthetasc. Boxplots indicate the variation of odorant concentration transported to the surface of the aesthetasc. Horizontal line within the box indicates the median, while the lower and upper edges of the box represent the 25 th and 75 th percentiles, respectively. Vertical lines extending from the box indicate the minimum and maximum measured values.figure.caption.26. Smaller 
boxplots indicate more uniform concentrations sampled along the circumference of the aesthetasc.figure.caption.27b). In addition, increased flushing efficiency occurs at further distal distances along the length of the aesthetasc (figure 2.13Flushing efficiency, quantified as the fraction of old molecules displaced by new molecules during subsequent flicks, plotted in relation to (a) aesthetasc spacing, (b) flick speed, and (c) position of sensing at different distal distances along the length of an aesthetasc. Boxplots are described in figure 2.12Variation of normalized mean concentration of odorant molecules around the aesthetasc surface with (a) odorant filaments of various width encountered during the beginning of the downstroke motion of a flick, (b) odor filaments of various width encountered during the end of the downstroke, (c) aesthetasc spacing, (d) flick speed, and (e) position of sensing at different distal distances along the length of an aesthetasc. Boxplots indicate the variation of odorant concentration transported to the surface of the aesthetasc. Horizontal line within the box indicates the median, while the lower and upper edges of the box represent the 25 th and 75th percentiles, respectively. Vertical lines extending from the box indicate the minimum and maximum measured values.figure.caption.26. Smaller boxplots indicate more uniform concentrations sampled along the circumference of the aesthetasc.figure.caption.27c). For all cases, increases in aesthetasc spacing, flick speed and distal distance along the aesthetasc decreases the variability of odorant concentrations sampled at different locations along the circumference of the aesthetasc.

Although increases in aesthetasc spacing and length, as well as flick speed increases the ability for crayfish to sample new odors, these animals must also be able to discretely sample their fluid environment. Therefore, a balance of these two competing processes is necessary for P. clarkii: to sample new odors during a downstroke flick but retain these odors over sufficient time periods during the returnstroke. Figure 2.14Variation of normalized odorant concentration with time during a flick-return sequence for different values of $g / d$ at $v=v_{0} \cdot g / d$ ratios for each line are labeled on the figure. ( $d$ is the typical gap between the aesthetascs and $v_{0}$ is the typical flick speed.)figure.caption.28 shows time courses of odorant 

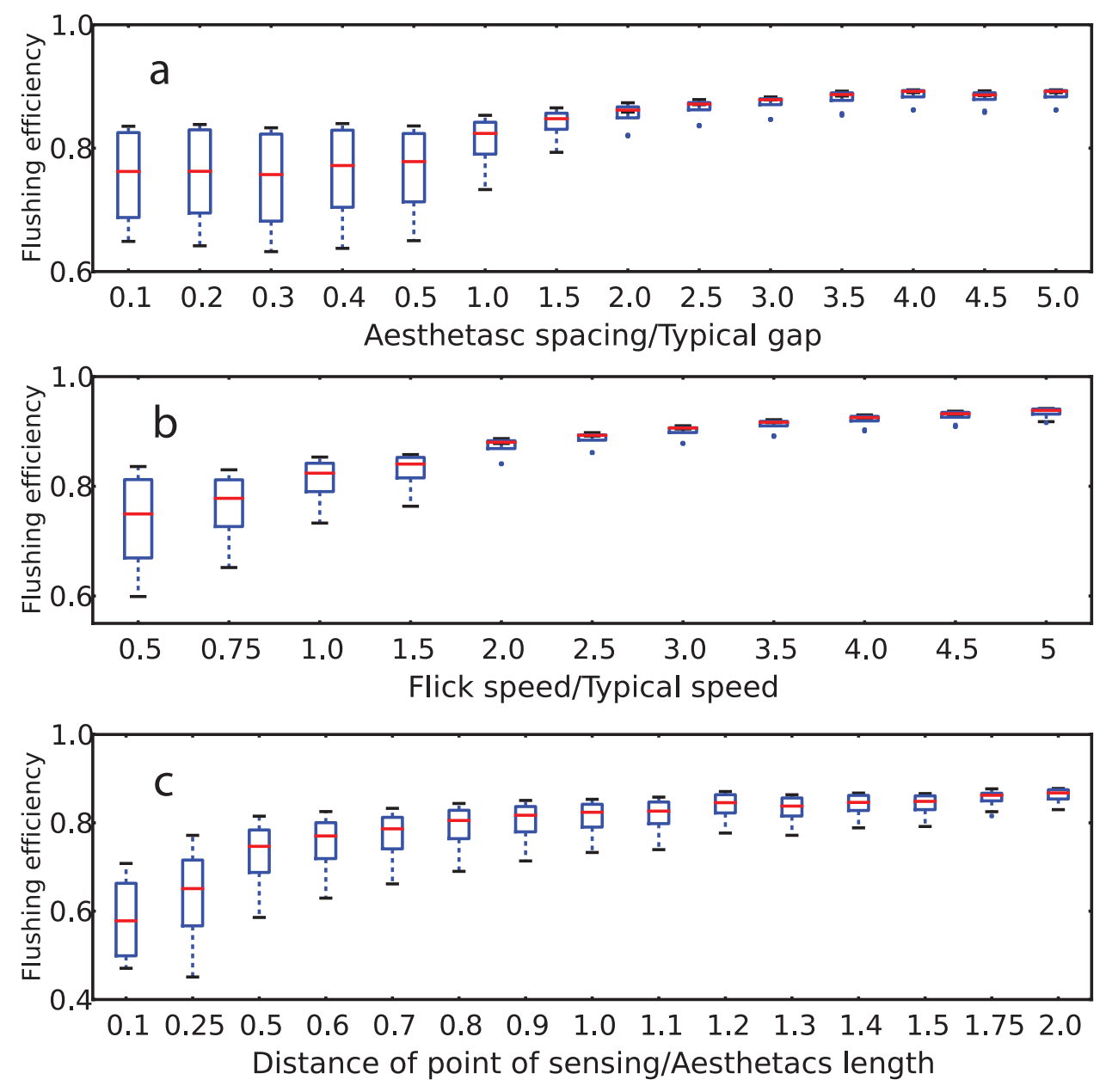

Figure 2.13: Flushing efficiency, quantified as the fraction of old molecules displaced by new molecules during subsequent flicks, plotted in relation to (a) aesthetasc spacing, (b) flick speed, and (c) position of sensing at different distal distances along the length of an aesthetasc. Boxplots are described in figure 2.12Variation of normalized mean concentration of odorant molecules around the aesthetasc surface with (a) odorant filaments of various width encountered during the beginning of the downstroke motion of a flick, (b) odor filaments of various width encountered during the end of the downstroke, (c) aesthetasc spacing, (d) flick speed, and (e) position of sensing at different distal distances along the length of an aesthetasc. Boxplots indicate the variation of odorant concentration transported to the surface of the aesthetasc. Horizontal line within the box indicates the median, while the lower and upper edges of the box represent the 25th and 75 th percentiles, respectively. Vertical lines extending from the box indicate the minimum and maximum measured values.figure.caption.26. Smaller boxplots indicate more uniform concentrations sampled along the circumference of the aesthetasc.

concentration along the aesthetasc surface for different values of aesthetasc spacing. For aesthetasc spacing of $g / d \geq 1$, these plots indicate odorant capture at concentrations near 


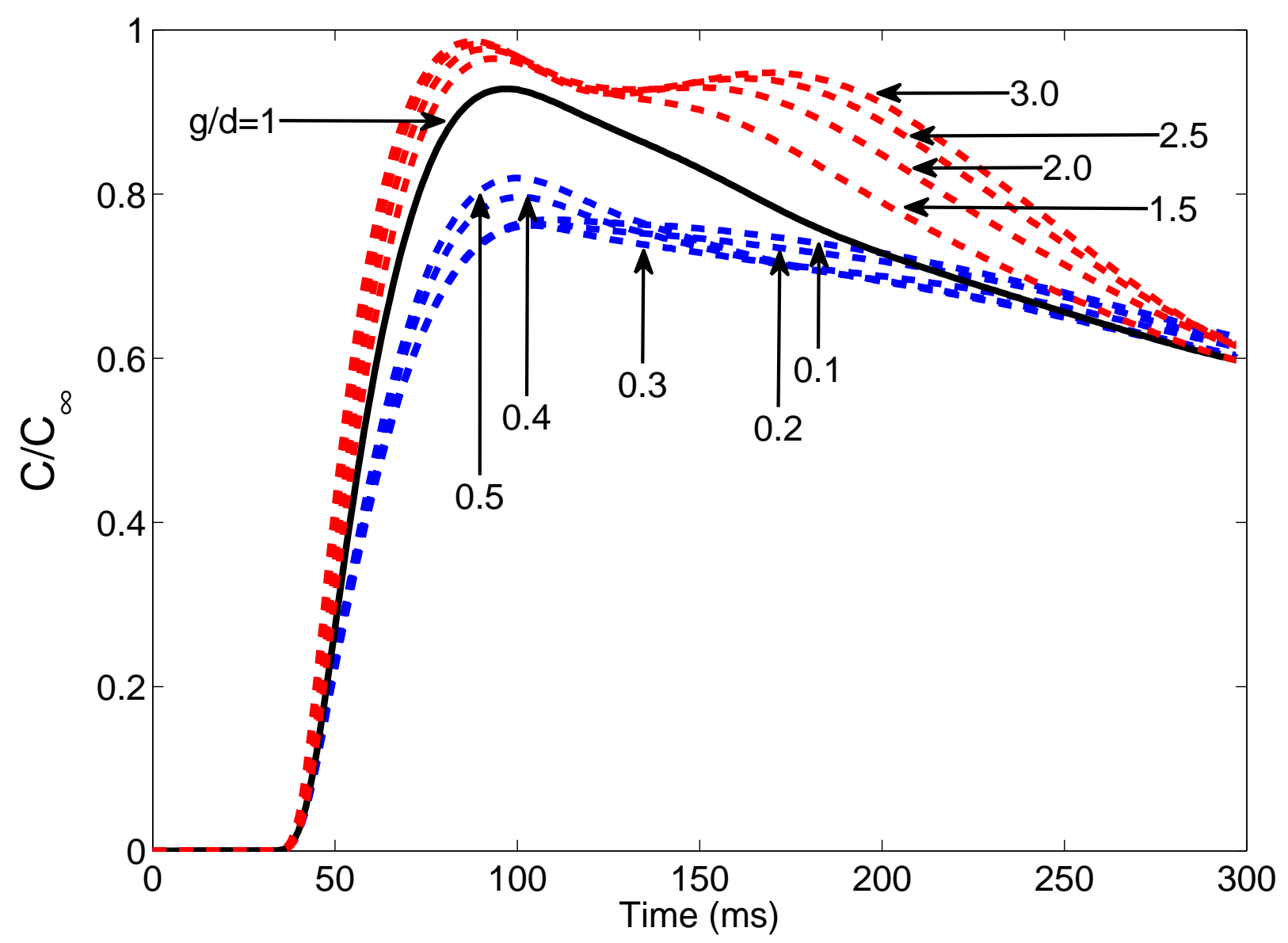

Figure 2.14: Variation of normalized odorant concentration with time during a flick-return sequence for different values of $g / d$ at $v=v_{0} . g / d$ ratios for each line are labeled on the figure. ( $d$ is the typical gap between the aesthetascs and $v_{0}$ is the typical flick speed.)

ambient levels $\left(C / C_{\infty}>0.9\right)$ at the end of the downstroke, with sharp declines in odorant capture for $g / d<1$. However, during the returnstroke (time periods $>100 \mathrm{~ms}$ ) larger gap spacings between aesthetascs show a greater loss of concentration, indicating a more 'leaky' returnstroke that prevents discrete sampling of the same odorant laden fluid sample. A balance between greater sampling of new odorants during the downstroke, with retention of these odorants during the returnstroke appears to occur with an aesthetasc spacing in the range of one hair diameter $(g / d=1)$.

Figure 2.15Variation of normalized odorant concentration with time during a flick-return sequence for different flick speeds at $g / d=1 . v / v_{0}$ ratios for each line are labeled on the 
figure.figure.caption.29 shows time courses of odorant concentration at the aesthetasc surface for different flick speeds. For faster flick speeds, these plots indicate odorant capture at concentrations near ambient levels $\left(C / C_{\infty}>0.9\right)$ at the end of the downstroke, with sharp declines in odorant capture for $v / v_{0}<1$ ( $v_{0}$ is the typical flick speed). However, during the returnstroke, large variations in odorant concentration are evident for fast flick speeds, indicating a more 'leaky' returnstroke that prevents discrete sampling of the same odorant laden fluid sample. Similar to $g / d$ spacing, a balance between greater sampling of new odorants during the downstroke, with retention of these odorants during the returnstroke, appears to occur at speeds similar to that at which P. clarkii have been observed to flick (i.e., $\left.v / v_{0}=1\right)$.

\subsection{Discussion}

A numerical model of the antennule and aesthetasc structure of the freshwater crayfish, P. clarkii, was developed to simulate the advective-diffusive transport of odorant molecules to aesthetasc surfaces. The first step in the process of sniffing is the interception by an olfactory organ of odorants in the surrounding air or water. Our results show that the rapid downstroke of the antennules facilitates transport of odorant molecules to the region surrounding the aesthetascs, while the slower returnstroke enables for additional time for the entrapped odorants to molecularly diffuse to the aesthetasc surfaces. The quantity of odorant molecules present at the aesthetasc surface reaches peak values near the end of downstroke, but due to the comparatively slow nature of the return stroke, significant concentrations of odorant are

retained in the vicinity of the aesthetascs at the end of the flick/return sequence. Subsequent flicks were shown to remove the odorants captured during the previous flick and capture new odorants from the ambient flow, at a replacement rate of up to $97.6 \%$. Thus, flicking provides a mechanism to discretely sample the odorant-laden fluid environment in both space and time while the animal navigates within a plume. Although early models of odorant tracking 


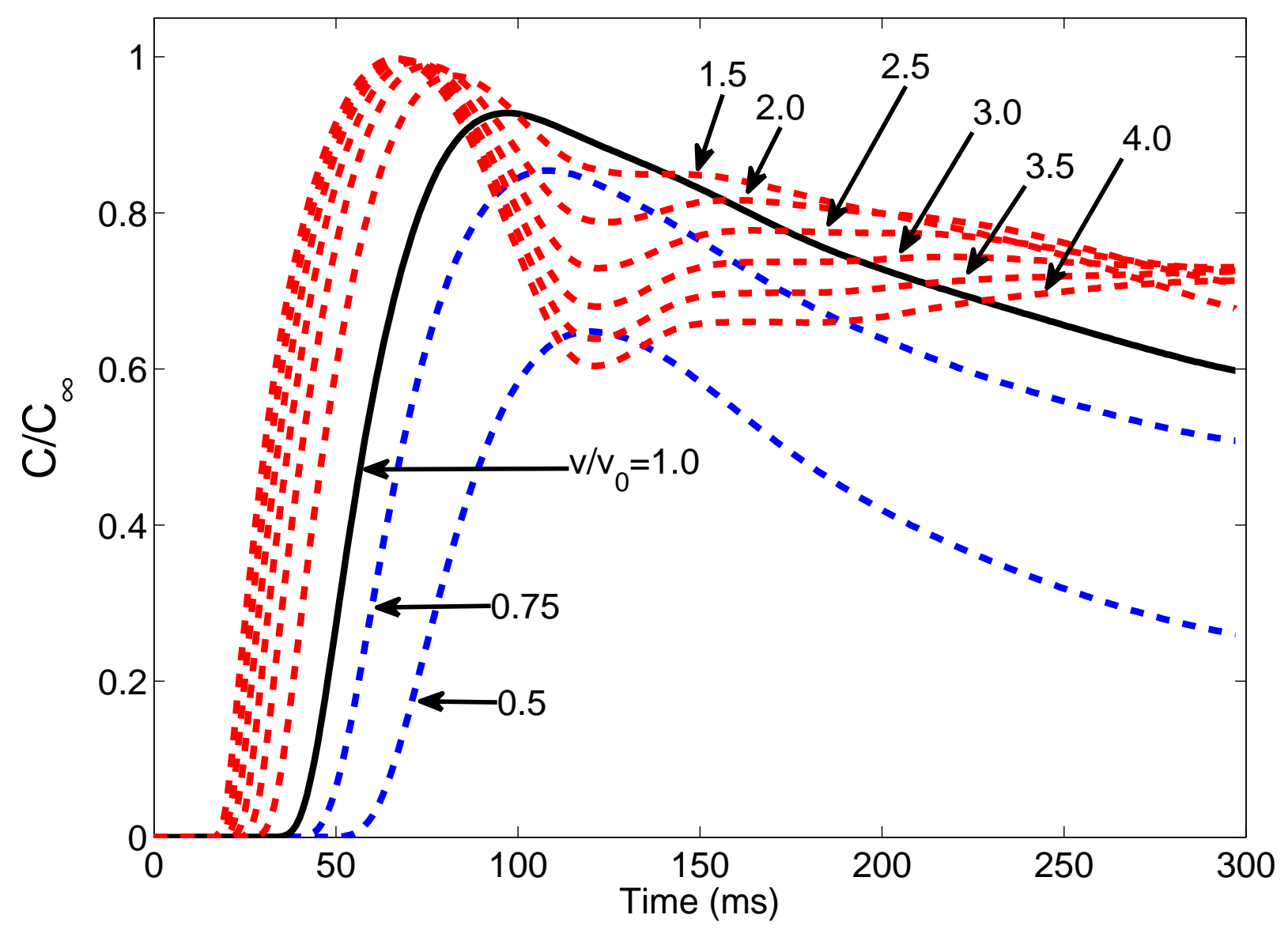

Figure 2.15: Variation of normalized odorant concentration with time during a flick-return sequence for different flick speeds at $g / d=1 . v / v_{0}$ ratios for each line are labeled on the figure.

assumed that animals respond to time-averaged concentration gradients in a plume [52], the rapidity of plume tracking maneuvers by crustaceans and insects suggests that discrete sampling of time-varying odorant concentrations is necessary to locate an odorant source $[53,8]$. The plume tracking success of crustaceans $[54,55]$ and insects [53] has been found to be lower in homogeneous plumes than in intermittent plumes. Likewise, the frequency of odorant encounters by crayfish affects their walking speed while searching for the source $[56,57]$. This suggests that successful plume tracking depends on discrete sampling of the filamentous structure of an odorant plume $[34,58,8]$ and flicking provides a mechanism to achieve this. Overall, for crayfish it is not known how long odorant molecules need to be 
in contact with chemosensory cells, or in what concentration to initiate a response. After reaching the surface of a sensillum, odorant molecules must still diffuse through the cuticle, through the extracellular space, and to receptors on the dendrites of olfactory receptor neurons. The patterns of action potentials that those neurons produce in response odorants, and processing of those signals in the brain, ultimately determines whether or not the odorants are detected by the animal $[41,59,60]$. For the spiny lobster, Panulirus argus, or a clawed lobster, Homarus americanus, when an odorant pulse is delivered to the aesthetascs, receptor neurons in the antennule respond (after a latency period of $70500 \mathrm{~ms}$ ) with a series of action potentials (spikes) that lasts $100200 \mathrm{~ms}$, followed by a period of decline in the frequency and amplitude of spikes $[61,62]$. Both the maximum spike frequency and total number of spikes correlate with the onset slope and amplitude of concentration change above background $[61,63,62,58]$. For the crayfish numerical model, peak concentrations at the aesthetasc surface were found to occur at approximately $50 \mathrm{~ms}$ after the initiation of the flick and odorant molecules remain in contact with the aesthetasc surface until the initiation of the subsequent flick. The duration over which animals are exposed to odorants is also important in neural coding. For example, the antennal receptor neurons in H. americanus require at least $50 \mathrm{~ms}$ of odorant stimulus to fire, but $\geq 200 \mathrm{~ms}$ for the spike frequency to reflect the concentration increase of the odorant pulse [61]. However, if an odorant stimulus lasts too long, chemoreceptor cells undergo adaptation, i.e. their response to another odorant pulse of the same or lower concentration is reduced or absent. Lobster antennule receptor neurons start to adapt after continuous exposure to an odorant stimulus of $300 \mathrm{~ms}$, and are completely adapted after $1000 \mathrm{~ms}$ exposure [64]. Such adaptation resets the sensitivity of the neuron such that it can respond to pulses of odorant concentration higher than background, thereby remaining sensitive to transient increases in odorant concentration in environments where the background concentration varies [65]. For the crayfish model, the aesthetascs were observed to encounter an odorant concentration of more than $90 \%$ ambient concentration at the end of the downstroke flick, and retain greater than $60 \%$ of the ambient concentration by the 
end of the returnstroke, thus enabling the aesthetasc to be exposed to concentration over the duration of the flick (approximately $240 \mathrm{~ms}$ ) and sufficient time for neurons to respond to the presence of odorants. Subsequent flicks showed exchange of these odorants with either new odorants, or no-odor for the case of flicking into odorant-free water, which may prevent P. clarkii neurons from undergoing adaptation to continual exposure to odorants on timescales of $300 \mathrm{~ms}$ and greater. The frequency of repeated odorant pulses can also affect neural responses. If an odorant stimulus starts $\geq 400 \mathrm{~ms}$ after the previous one ends, antennule olfactory receptor cells of $\mathrm{H}$. americanus can resolve them as separate pulses, but if it arrives $\leq 200 \mathrm{~ms}$ after the previous odorant has stopped, then the neuron responds as though the second pulse is a continuation of the previous one [66]. The bursts of firing by antennule olfactory receptor neurons of P. argus adapt selectively to odorant pulses at frequencies of 0.5-0.1 Hz [62]. The flicking rate of the crayfish was measured to be 3.3 flicks s ${ }^{-1}$, which falls within the minimum range that aquatic organisms need to be exposed to odorants of $50 \mathrm{~ms}$, but less than the time at which adaption to odorants begins to occur of $300 \mathrm{~ms}$ [61]. Taken together, intermittency in odorant sampling is important to both initiate receptor neuron responses to odorants, and to limit adaptation to these odorants.

\subsubsection{Aesthetasc spacing and length}

Both the antennule morphology and flicking kinematics of P. clarkii were altered through changes in the numerical model to determine their impacts on odorant transport to aesthetasc surfaces. The variation of the spacing between adjacent aesthetascs has a noticeable effect on variations in concentration levels in the region between the aesthetascs, as well as the flux of odorants to their surfaces. The aesthetascs are typically spaced one aesthetasc diameter apart. An aesthetasc gap width greater than one diameter allows larger quantities of odorant molecules to pass between them during the downstroke, but also increases the 'leakiness' of these trapped odorants during the returnstroke [67]. Leakiness becomes important with regard to how morphology and velocity impact delivery of odorants to sensory cells. When 
leakiness is large, boundary layers are reduced as water is allowed to pass through adjacent hairs. This enhanced odorant transport decreases the distance across which diffusion must occur to reach sensory cells. However, for antennules exposed to high ambient currents, such a system would not allow for discrete sampling of odorants during a flick. Interestingly, no matter how wide the gap, odorant concentrations at the aesthetasc surface never reach $100 \%$ ambient concentration during the flick/return sequence, suggesting that although the thickness of the diffusive boundary layer is reduced during the flick, it is not removed entirely. Various locations along the length of the aesthetasc, ranging from points near the base to well beyond the average aesthetasc length for a hypothetically longer aesthetasc, were tested to determine the effect of location on the aesthetasc on odorant delivery. Results revealed that larger amounts of molecules were entrapped towards the distal two-thirds of the aesthetasc, where there is a transparent cuticle that is thought to allow rapid transport of odorants to chemosensory cells [68], than along the proximal end close to the attachment point along the antennule. In addition, enhanced odorant capture occurs with longer aesthetascs beyond the typical aesthetasc length, suggesting rapid breakdown of boundary layers near the tip, which facilitates greater exposure of the aesthetasc surface to incoming odorants. However, again, this enhances 'leakiness' of the flow between aesthetascs during the returnstroke, limiting the ability of the olfactory organ to take discrete samples of odorant-laden fluid.

\subsubsection{Flick speed}

The number of odorant molecules encountered by the aesthetasc per unit of its surface area increases with $R e$ of the flick, even though the aesthetasc experiences the odorant flux for a longer duration for slower flick speeds. The flick speed, and hence the Reynolds number, both have direct impact on the flow of odorants through the aesthetasc arrays and how rapidly the odorant molecules contact the aesthetasc surface. At high flick speeds, where rates are greater than double typical speeds, odorant concentrations along the aesthetasc surface increase quickly, and near the end of the downstroke, a pronounced flow reversal 
occurs, causing a large proportion of the odorant molecules present between the aesthetascs to get washed away during the returnstroke. However, for slower speeds, this flow reversal does not appreciably occur and odorants are more effectively 'trapped' within the aesthetasc hair array throughout the flick sequence. Although a wide diversity of antennule morphologies and flick speeds occur across many species that allow animals to take discrete samples of their fluid environment $[11,46]$, our findings indicate that P. clarkii flicks its antennules at speeds which maximizes the difference in fluid leakiness between the downstroke and returnstroke. In addition, our results extend this idea to show (in fiures 2.14Variation of normalized odorant concentration with time during a flick-return sequence for different values of $g / d$ at $v=v_{0}$. $g / d$ ratios for each line are labeled on the figure. $(d$ is the typical gap between the aesthetascs and $v_{0}$ is the typical flick speed.)figure.caption.28 and 2.15Variation of normalized odorant concentration with time during a flick-return sequence for different flick speeds at $g / d=1$. $v / v_{0}$ ratios for each line are labeled on the figure.figure.caption.29) that this discrete fluid sampling maximizes odorant delivery to aesthetasc surfaces during the flick/return sequence.

\subsubsection{Odorant filament width}

Changes in odorant filament width and their impact on transport of odorants suggest that very low concentrations, relative to ambient levels, are encountered along aesthetasc surfaces for thin odorant filaments. For odorant filaments of thickness $1 \mathrm{~mm}$ or less (i.e., less than $50 \%$ of the flick distance of $2 \mathrm{~mm}$ ), concentrations of $C \leq 0.5 C_{\infty}$ was encountered along the aesthetasc surface, regardless of whether odorant filaments were positioned at the start or the end of the downstroke. For wider filaments, high concentrations are encountered and asymptote to peak concentration levels for filaments with a $2 \mathrm{~mm}$ thickness; close to the distance over which a flick spans. At these 1-2 mm filament thicknesses, filaments encountered at the beginning of a flick capture more molecules than filaments encountered at the end of the downstroke. 


\subsubsection{Conclusions regarding antennule flicking and odorant detec- tion by $P$. clarkii}

Behavioral studies have indicated that the instantaneous spatial distribution of odorants sampled by olfactory organs at different locations within a plume can provide important cues for tracking $[8,69,70]$. The intermittency in odorant encounters, the duration over which animals are exposed to odorants, and changes in the onset slope of concentrations along the aesthetasc surface all appear to be important parameters that impact the response of organisms to its odorant environment [63]. Our results suggest that flicking (1) enhances the ability for crayfish to discretely sample their odor environment due to rapid replacement of odorants during successive flicks, (2) offsets adaptation of chemosensory neurons that may occur due to continued exposure to odorants and (3) reduces the chance of flicker-fusion, where two different odorant filaments are perceived as one [64]. The spike response of the ORN neuronal targets within the brain to the changes in concentrations and odorant encounter rates have been documented for crayfish [49] and findings from our numerical model suggest that ORN responses are directly impacted by morphological parameters and flow scenarios that control the transport of odorant molecules to aesthetasc surfaces. The receptor neurons located within aesthetascs respond to concentration gradients in a turbulent plume by producing a series of action potential spikes. The spike pattern that these neurons produce in response to odorants, along with processing of these signals in the brain, determines whether odorants are detected by the animal [71, 60]. The spike frequency is higher for steeper concentration gradients, but spike counts revert to zero or low levels during exposure to persistently high odorant concentrations [63], suggesting swift adaptation

of chemoreceptor cells to background concentration levels. As found in this study, increasing the gap spacing between aesthetascs, increasing the flick speed, and increasing aesthetasc length each create (1) quicker attainment of peak concentrations, (2) higher concentrations surrounding the aesthetascs and (3) a more uniform distribution of odorants around the aesthetasc surface. These changes to antennule morphology and kinematics increase exposure 
to ambient odorants, but may also provide poor conditions for discrete sampling of the odor environment or cause adaptation of chemoreceptor cells. Our results also suggest that through changes in both the timing and encounter rate of odorant molecules to aesthetasc surfaces caused by antennule flicking, odorant filaments of varying thickness and concentration can be distinguished, which has been shown to vary in systematic ways with distance from the source $[14,69]$. Therefore, for P. clarkii both the sampling behavior and neural responses to odorants involved in olfaction appear well suited to the intermittent spatial and temporal distribution of odorants within the environment. 


\title{
CHAPTER 3
}

\section{Simultaneous sampling of flow and}

\section{odorants by crustaceans can aid}

\section{search within a turbulent plume}

A part of this chapter has been published, with citation: Pravin, Swapnil, and Matthew A. Reidenbach. "Simultaneous Sampling of Flow and Odorants by Crustaceans can Aid Searches within a Turbulent Plume." Sensors 13.12 (2013): 16591-16610.

\begin{abstract}
Crustaceans such as crabs, lobsters and crayfish use dispersing odorant molecules to determine the location of predators, prey, potential mates and habitat $[1,2,3,4,5,6]$. Odorant molecules diffuse in turbulent flows and are sensed by the olfactory organs of these animals, often using a flicking motion of their antennules. These antennules contain both chemosensory and mechanosensory sensilla, which enable them to detect both flow and odorants during a flick. To determine how simultaneous flow and odorant sampling can aid in search behavior, a 3-dimensional numerical model for the near-bed flow environment was created. A stream of odorant concentration was released into the flow creating a turbulent plume, and both
\end{abstract}


temporally and spatially fluctuating velocity and odorant concentration were quantified. The plume characteristics show close resemblance to experimental measurements within a large laboratory flume. Results show that mean odorant concentration and its intermittency, computed as $d c / d t$, increase towards the plume source, but the temporal and spatial rate of this increase is slow and suggests that long measurement times would be necessary to be useful for chemosensory guidance. Odorant fluxes measured transverse to the mean flow direction, quantified as the product of the instantaneous fluctuation in concentration and velocity, $v^{\prime} c^{\prime}$, do show statistically distinct magnitude and directional information on either side of a plume centerline over integration times of $<0.5 \mathrm{~s}$. Aquatic animals typically have neural responses to odorant and velocity fields at rates between 50 and $500 \mathrm{~ms}$, suggesting this simultaneous sampling of both flow and concentration in a turbulent plume can aid in source tracking on timescales relevant to aquatic animals.

\subsection{Introduction}

\subsubsection{Chemosensing in Aquatic Animals}

Crustaceans such as crabs, lobsters and crayfish use chemo- and mechano-reception to track sources of odorant plumes to locate mates, food, and living habitat $[1,2,3,4,5,6]$. Odorants in the benthic flow are carried to the olfactory organs of the animal through turbulent water currents and diffuse toward the surface of the organs where chemoreceptors are located. These olfactory organs also contain mechano-receptors that provide information about the turbulent flow, and together with odorant concentration help the animal locate the source of the chemical plume [7]. Animals use a variety of sensing strategies to orient themselves in the direction of the plume source depending on the flow regimes they operate in [8]. Hence, to understand the mechanism of chemical plume tracking in aquatic animals, we must understand not only the small scale diffusive flow of odorants near the olfactory organs of the animals, but also the large scale turbulent nature of the chemical plume. Crustaceans have 
olfactory appendages called antennules, which bear tiny hair-like structures called aesthetascs (Figure 3.1(A) The freshwater crayfish, Procambarus clarkii, with lateral antennule labeled. Grid in the background is 1 x $1 \mathrm{~cm}$; (B) Scanning electron micrograph (SEM) of the lateral antennule with (a) chemosensory aesthetascs and (b) mechanosensory sensilla labeled (photo D. Mellon); (C) The spiny lobster, Panulirus argus, with the lateral antennule labeled; (D) SEM of the lateral antennule with (a) chemosensory aesthetascs; (b) mechanosensory sensilla and (c) guard hairs labeled (photo J.A. Goldman).figure.caption.31). The aesthetascs are often covered by a permeable cuticle membrane underneath which reside chemoreceptors. The chemoreceptors contained on the aesthetascs are composed of dendrites (branched projections) of olfactory receptor neurons (ORNs), which send information, through electrical impulses, to the olfactory lobes of the brain [9]. The diffusion of odorant molecules toward and through the cuticle membrane is responsible for delivering the odorants to these chemoreceptors. To facilitate the transport of odorant laden flow to the antennules, many aquatic animals use flicking or fanning of their appendages [5, 10]. This behavior is often described as sniffing. A flicking motion often involves a fast down-stroke and a slower return-stroke, leading to entrapment of odorant molecules between the aesthetascs, which lowers the diffusion time of odorants to the aesthetasc surface and enables these animals to discretely sample their ambient environment $[11,12]$. The odorant plumes encountered in the environment of these organisms are often turbulent and highly filamentous in nature [6, 14]. Due to stirring by the turbulent motion of the fluid, the spatial and temporal distribution of odors is complex and filaments of high odor concentration are often adjacent to little or no odorants $[14,15]$. These distributions in odorants also change in response to variations in the ambient flow speed and bed roughness, where the variance in odorant fluctuations is reduced for rougher beds [16] and greater mean velocities [14]. Certain cues, such as correlations between the flow kinematics and odorant concentration that the animal sense through the chemoreceptors and mechanoreceptors, can provide valuable information regarding the plume source. However, due to the high intermittency and temporal and spatial variability of the plume, this often 


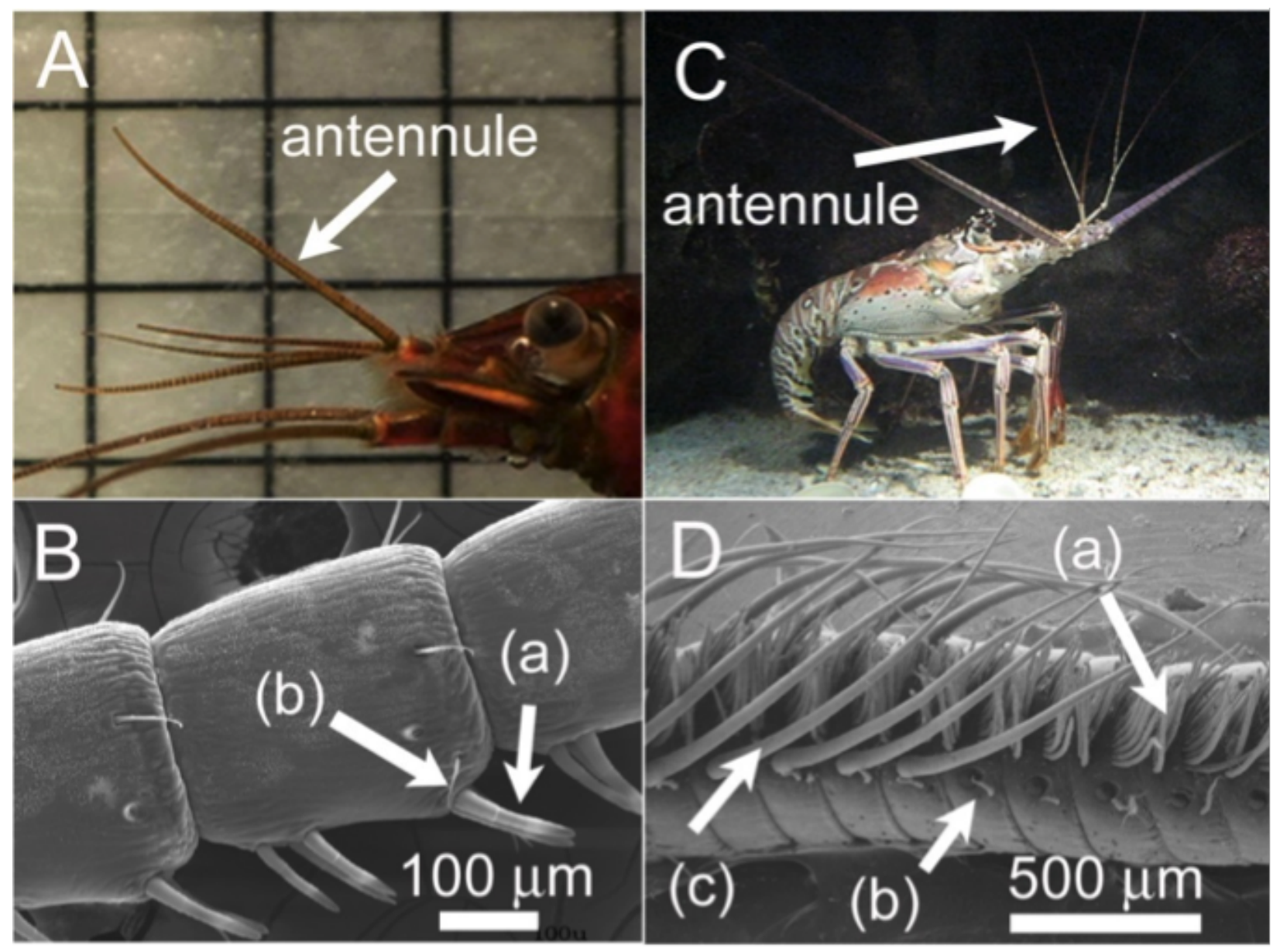

Figure 3.1: (A) The freshwater crayfish, Procambarus clarkii, with lateral antennule labeled. Grid in the background is $1 \times 1 \mathrm{~cm}$; (B) Scanning electron micrograph (SEM) of the lateral antennule with (a) chemosensory aesthetascs and (b) mechanosensory sensilla labeled (photo D. Mellon); (C) The spiny lobster, Panulirus argus, with the lateral antennule labeled; (D) SEM of the lateral antennule with (a) chemosensory aesthetascs; (b) mechanosensory sensilla and (c) guard hairs labeled (photo J.A. Goldman).

reduces the ability of organisms, such as the blue crab, Callinectes sapidus, to successfully navigate to the source of an attractive odor [17].

\subsubsection{Sampling Rates and Tracking Strategies in Crustaceans}

The frequency of flicking and sensitivity to odorants can alter the tracking strategy in the animals. Higher sampling frequency allows an animal to sample a larger number of odorant filaments as it moves through the plume [14]. Blue crabs C. sapidus, spiny lobsters 
Panulirus argus and freshwater crayfish Procambarus clarkii all flick their antennules at a rate of approximately $3 \mathrm{~Hz}$, but can vary between 0.5 and $4 \mathrm{~Hz}[14,30]$. Although it was previously assumed that most odor tracking by animals occurred by responding to time-averaged concentration gradients in a plume [52], the speed at which plume-tracking maneuvers occur suggest that more instantaneous sensory feedbacks are being utilized $[8,53]$, and that time-averaged concentrations converge too slowly to be useful to a foraging animal [42]. In a similar fashion, it was also found that resolving the rise slope of concentration bursts requires sampling rates that are too fast for animals and the spatial variation in the rise slope is too small to be utilized in search without long sampling periods. Overall, the time-averaged concentration, rise slope, and burst shape of concentration filaments, if utilized alone, have limited usefulness for plume tracking [42]. Page et al. [57] found that in blue crabs, odorants elicit responses in a binary way, causing upstream motion provided that the concentration detected along the antennules exceeds a specific threshold. However, this threshold was different for each crab as well as different due to the prior stimulus history, suggesting a context-sensitive response to signal dynamics. In addition, Page et al. [72] found that the spatial distribution of the odorant concentration field was utilized to correct for cross-stream motion, and that crab movement is continually adjusted to maintain an upstream heading in response to both the concentration and its distribution. While many

organisms using this flicking of their antennules to discretely sample the plume at short timescales [73], some slower moving predators such as the knobbed whelk Busycon carica have been found to use temporal averaging to gather information on the spatial extent of the plume [74]. Whether this temporal averaging is used widely by aquatic organisms, or how useful it is within highly turbulent plumes, is still unknown.

\subsubsection{Neural Responses to Odorant and Flow Information}

Previous studies have shown that the time course of advection and molecular diffusion of odorants to chemoreceptors can play a significant role in neural responses [19, 21]. For 
example, the olfactory receptor neurons in the lobster Homarus americanus require at least $50 \mathrm{~ms}$ of odorant stimulus to fire, but $200 \mathrm{~ms}$ or more for the spike frequency to fully respond to the concentration of the odor pulse [61]. However, lobster antennule receptor neurons start to adapt after continuous exposure to an odor stimulus of $300 \mathrm{~ms}$ and are completely adapted after 1,000 ms of exposure. This adaptation resets the sensitivity of neuron response to odorant concentration higher than background levels. Neuron responses in the spiny lobster Panulirus argus show decreased sensitivity when continually exposed to odorants at repeated odor pulses between $100 \mathrm{~ms}$ to $500 \mathrm{~ms}$ [62]. This suggests that both intermittency in the odorant signal within the plume and discrete sampling of odorants by the animal are highly beneficial for detection of odorants and affect the rate of receptor neuron firing $[61,62]$. Hydrodynamic stimulation of the antennules, including flicking [75, 32] and from ambient current evokes electrical activity in the central brain neurons $[68,49,48]$. However, peak responses of olfactory receptor neurons occur not solely when the aesthetasc responds to odorants, but rather in conjunction with a hydrodynamic stimulus [68, 76, 77]. Thus, concentration and flow cues simultaneously excite chemoreceptors and mechanoreceptors of the antennules during flicking. In addition, many invertebrate organisms, including the spiny lobster P. argus [78], and crayfish P. clarkii [7], contain bimodal chemo-mechanoreceptors on their antennules that respond to both odorants and flow. For P. clarkii, local deutocerebral interneurons integrate hydrodynamic and odorant inputs, and the response of these central elements is enhanced when both flow and odorant stimulation occurs together [68]. For the crayfish Orconectes virilis changes in the temporal pattern of odorant stimulation was found to drive changes in the temporal patterns of behavior while undergoing search [79]. It has also been shown in blue crabs Callinectes sapidus that both rheotaxis and chemotaxis is necessary for successful orientation while tracking food odors [80]. These findings suggest that these organisms utilize both hydrodynamic and chemical stimuli to aid in search behavior, but what chemical and flow information these organisms use, and how it is integrated at the level of the antennule or brain remains poorly understood. 


\subsubsection{Research Objectives}

The objective of this study is to determine the impact of ambient flow environments on turbulent mixing and odorant transport within a chemical plume, and how crustaceans might use simultaneous flow and odorant information to orient themselves within a plume. The specific questions that are addressed in this study are: (1) How does bed roughness and water velocity impact turbulence structure and mixing of dissolved odorants? (2) What is the effect of flow kinematics on mean and instantaneous flux of odorants at different downstream and transverse locations within a turbulent plume? (3) Since antennules of aquatic crustaceans contain both chemo- and mechano-sensory sensilla, can they conceivably integrate velocity and concentration information to aid in finding the location of the plume source? We utilize simultaneous measurements of water velocities and odorant concentrations over a sand bed roughness within a large laboratory flume, as well as numerically simulate a similar turbulent plume using a 3-dimensional computational fluid dynamics model to address these questions. Understanding the potential mechanisms by which these organisms locate sources of odor can also provide inspiration for artificial bio-mimetic sensors.

\subsection{Methods}

\subsubsection{Flume Study}

The experimental data for flow and odorant structure within a turbulent plume were analyzed from a study conducted within a rectangular laboratory flume (Figure 3.2Recirculating flume with PLIF/PIV laser system. A $532 \mathrm{~nm}$ laser was used for particle image velocimetry, while a $488 \mathrm{~nm}$ laser was used to excite fluorescein dye for use in PLIF imaging. Images were obtained $13 \mathrm{~m}$ downstream from the leading edge of the flume and $1 \mathrm{~m}$ downstream from the source release of fluorescein dye.figure.caption.32) with dimensions $25 \mathrm{~m}$ long, $0.6 \mathrm{~m}$ wide, and $0.3 \mathrm{~m}$ high [81]. Velocity measurements were made using a Particle Image Velocimetry 
(PIV) system while concentrations were sequentially obtained using a coordinated planar laser-induced fluorescence system (PLIF). The PLIF technique, described in further detail in [14], uses fluorescein dye as the scalar tracer. A laser with an output wavelength of light at $488 \mathrm{~nm}$ was first passed through a beam expander, then a beam focus in order to sharpen the beam. The beam was then converted to a vertical sheet of laser light using a scanning moving-magnet mirror (Cambridge Technology Inc., Bedford, MA, USA). The frequency of laser light was within the absorption spectrum of fluorescein (mean excitation at $490 \mathrm{~nm}$ ), which excited the dye and emitted light at a mean wavelength of $520 \mathrm{~nm}$. Using a digital camera (1M60, Dalsa, Waterloo, Ontario, Canada), 1 megapixel, 12 bit resolution, 50 frames $\mathrm{s}^{-1}$ ), the fluoresced dye was imaged. The camera was fitted with an optical longpass filter which passed all light above 517 nm (Omega Optical Corp., Brattleboro, VT, USA), and therefore only emitted light from the fluoresced dye was imaged, while the ambient laser light was blocked. Imaging occurred over an approximate $8 \mathrm{~cm} \mathrm{x} 8 \mathrm{~cm}$ area $1 \mathrm{~m}$ downstream from the point of odorant release along the centerline of the plume. The laser was scanned to illuminate the imaging field every $0.04 \mathrm{~s}(25 \mathrm{~Hz})$, with a wait period of $0.04 \mathrm{~s}$ between scans. Raw images were processed to remove biases in the data, including varying pixel dark response, slow background changes in $\mathrm{pH}$ and temperature, lens and optics aberrations, and laser attenuation due to background concentrations [82]. A second laser with an output wavelength of light at $532 \mathrm{~nm}$ was used for PIV imaging. PIV is a standard technique [83] in fluid flow applications in which a thin sheet of laser light illuminates a two dimensional plane within a flow. Silver coated hollow glass spheres (diameter $=11 \mu \mathrm{m}$ ) were added to both the dye and bulk flow and the $532 \mathrm{~nm}$ laser was used to illuminate the particles. Since the $532 \mathrm{~nm}$ laser light was outside the absorption spectrum of fluorescein dye, the dye did not fluoresce. The laser was also pulsed at $0.04 \mathrm{~s}$ intervals, alternating in time with the PLIF laser scanning. Particle motions illuminated by the PIV laser were recorded with the same camera as the PLIF images in alternating frames [84]. Images of particle trajectories were analyzed using a numerical method that employs cross-correlation analysis to calculate the 


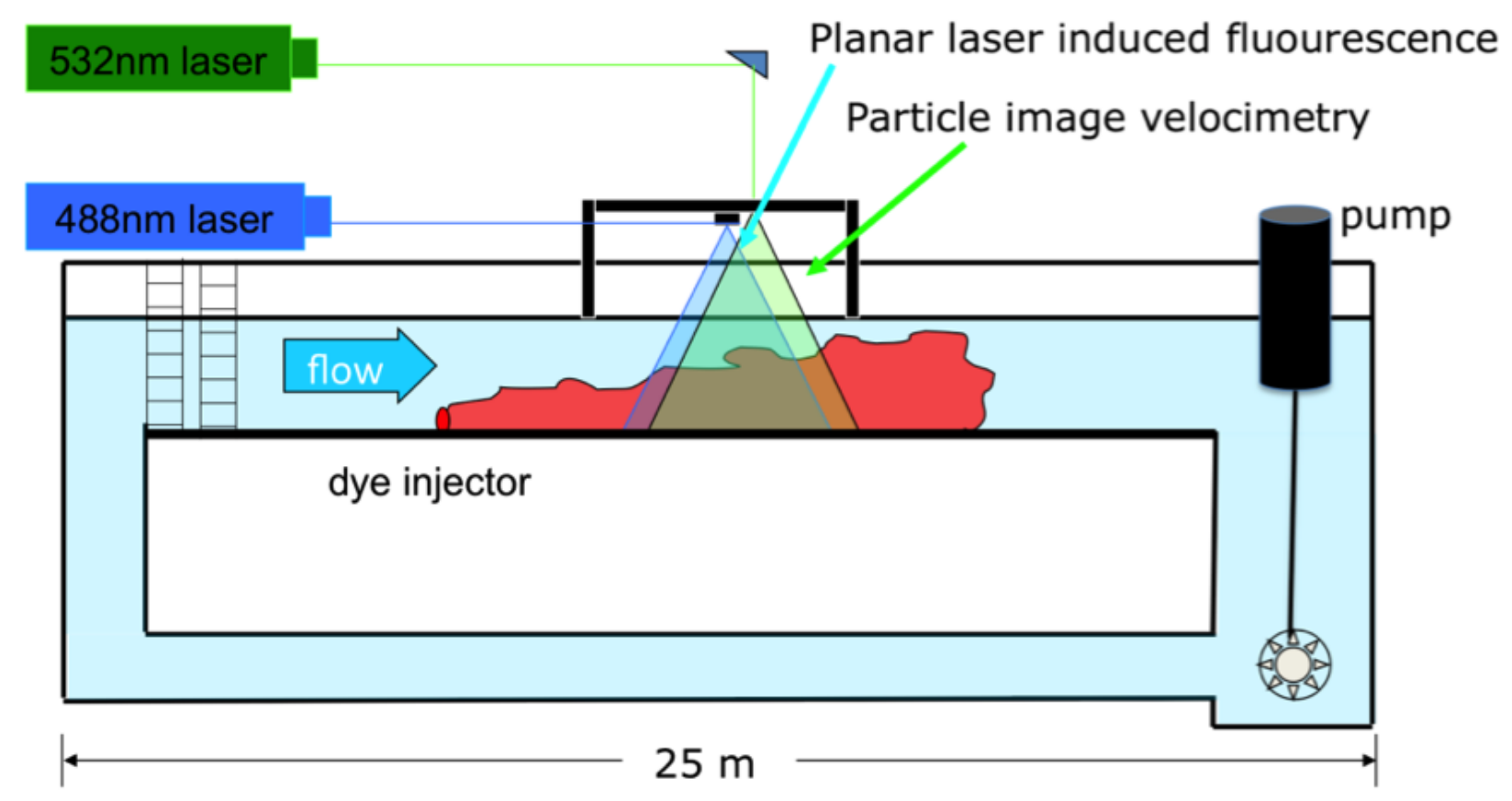

Figure 3.2: Recirculating flume with PLIF/PIV laser system. A $532 \mathrm{~nm}$ laser was used for particle image velocimetry, while a $488 \mathrm{~nm}$ laser was used to excite fluorescein dye for use in PLIF imaging. Images were obtained $13 \mathrm{~m}$ downstream from the leading edge of the flume and $1 \mathrm{~m}$ downstream from the source release of fluorescein dye.

most likely displacement of particles over a given time period between image frames [85]. The MatPiv 1.6.1 software package [86] was used to calculate velocity vectors for every 8 by 8 pixel subwindow, giving a velocity resolution to $0.6 \mathrm{~mm}$, while concentration information was on the pixel scale, with a resolution of $<0.1 \mathrm{~mm}$. Vector fields were superimposed on the sequentially-recorded scalar concentration field.

A dye source concentration Csource $=100 \mu \mathrm{g} \mathrm{cm}-3$ was used as the tracer. Fluorescein has a Schmidt number, $\mathrm{Sc}=1,970$ at $20^{\circ} \mathrm{C}$. Most odors which attract aquatic organisms are composed of amino acids with a molecular diffusivity, $\mathrm{D} \approx 10^{-9} \mathrm{~m}^{2} \mathrm{~s}^{-1}$, making fluorescein, with a molecular diffusivity of $\mathrm{D}=0.5 \times 10^{-9} \mathrm{~m}^{2} \mathrm{~s}^{-1}$ a good choice as a surrogate to model odor transport. Dye was injected uniformly across the width of the flume along the sediment-water interface using a line source diffuser. The diffuser, constructed of $1 \mathrm{~cm}$ diameter Tygon ${ }^{\circledR}$ tubing, released dye from $2 \mathrm{~mm}$ diameter ports spaced evenly in $2 \mathrm{~cm}$ increments across the width of the plume. Fluorescein input was from a constant head tank located above the 


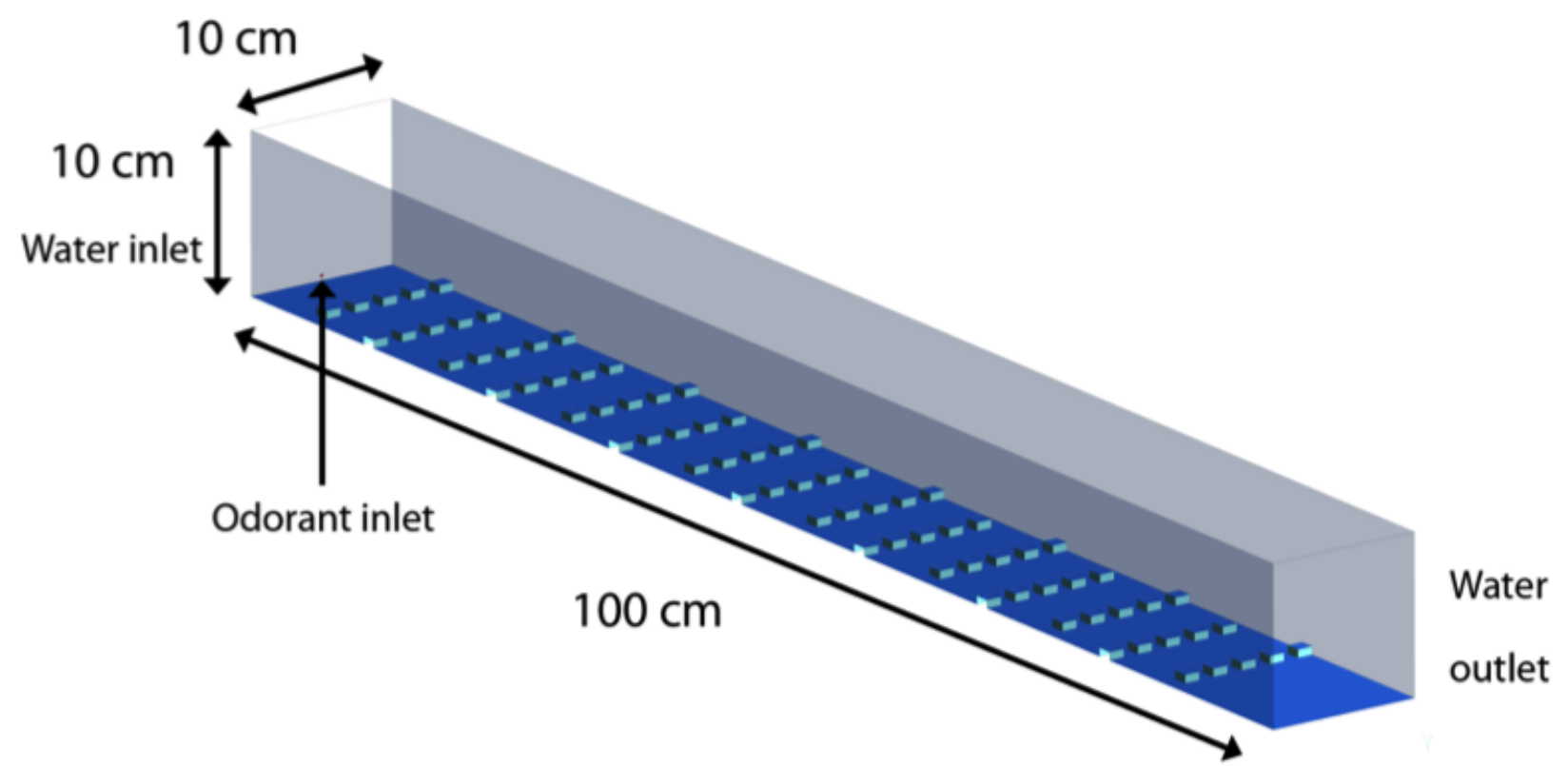

Figure 3.3: Schematics of the CFD domain for flow simulation. The bottom surface of the three-dimensional domain has cubical roughness elements placed in a staggered fashion. Water enters from the left end and exits from the right. The roughness humps at the bottom, of side length $1 \mathrm{~cm}$ and separated by $5 \mathrm{~cm}$ from each other, help trip the boundary layer and facilitate mixing leading to a turbulent flow downstream. A stream of odorants is released into the inflow at the odorant inlet on the left at an elevation of $z=0.5 \mathrm{~cm}$. The cubical humps have a base area of $1 \mathrm{~cm}$ by $1 \mathrm{~cm}$ and a height of $0.5 \mathrm{~cm}$ to hydraulically reproduce a sand bed roughness.

flume at a rate of $0.04 \mathrm{~L} \mathrm{~min}^{-1}$. This type of release created a uniform distribution of dye across the width of the flume while minimizing flow disturbances and mixing effects due to introduction of the dye. Measurements were made at $1 \mathrm{~m}$ downstream from the source. One flow condition, of approximately $U=10 \mathrm{~cm} \mathrm{~s}^{-1}$ was quantified over a bed composed of sand with a mean grain size diameter of $1.5 \mathrm{~mm}$. Due to memory capacity of the camera, 1,000 sequential images were collected in each run, equivalent to $20 \mathrm{~s}$ at a $50 \mathrm{~Hz}$ sampling rate. 10 runs were completed for each flow condition, therefore 10,000 total images were obtained, containing 5,000 PIV images and 5,000 PLIF images. 


\subsubsection{Numerical Model}

To simulate the turbulent plume measured within the laboratory flume, a 3-dimensional numerical model was developed that uses cubical roughness elements placed at the bottom surface of the flume to generate turbulence characteristics within the water column similar to the sand bed topography of the flume experiments. The cubical roughness elements were $0.5 \mathrm{~cm}$ high and the distance between successive rows of roughness elements was $5 \mathrm{~cm}$. Each successive row was placed in a staggered arrangement with respect to the previous row allowing continuous water movement in the region between the roughness elements. This creates a roughness height $(h)$ to spacing $(d)$ ratio of $d / h=10$, forming a k-type roughness, similar to that created by the sand bed [87]. Figure 3.3Schematics of the CFD domain for flow simulation. The bottom surface of the three-dimensional domain has cubical roughness elements placed in a staggered fashion. Water enters from the left end and exits from the right. The roughness humps at the bottom, of side length $1 \mathrm{~cm}$ and separated by $5 \mathrm{~cm}$ from each other, help trip the boundary layer and facilitate mixing leading to a turbulent flow downstream. A stream of odorants is released into the inflow at the odorant inlet on the left at an elevation of $z=0.5 \mathrm{~cm}$. The cubical humps have a base area of $1 \mathrm{~cm}$ by $1 \mathrm{~cm}$ and a height of $0.5 \mathrm{~cm}$ to hydraulically reproduce a sand bed roughness.figure.caption.33 shows the flow domain where water enters the left end of the flume and exits from the right end. A plume of odorants was injected into the flow domain from the left boundary, $3 \mathrm{~mm}$ above the bed, through a $2 \mathrm{~mm}$ wide opening. The grid size for the mesh varied throughout the domain to allow for a fine resolution of boundary layer flow near the roughness elements. The smallest grid size was $0.05 \mathrm{~cm}$ near the surface of the roughness elements. A large-eddy simulation (LES) technique was used for modeling turbulence in the flow, which allowed for accurate resolution of large eddies, while the Smagorinsky eddy-viscosity model was used for modeling small scale eddies. The governing equation for the flow is given by the Navier-Stokes equation: 


$$
\frac{\partial u_{i}}{\partial t}+u_{j} \frac{\partial u_{i}}{\partial x_{j}}=-\frac{1}{\rho} \frac{\partial p}{\partial x_{i}}+\nu \frac{\partial^{2} u_{i}}{\partial x_{j} \partial x_{j}}
$$

where $\rho$ is the density and $\nu$ is the dynamic viscosity of water. The continuity equation for incompressible flow, along with the equation above, defined the velocity field:

$$
\frac{\partial u_{i}}{\partial x_{i}}=0
$$

Using velocity data, the odorant concentration, $c$ was calculated at each time-step using the mass transport equation:

$$
\frac{\partial c}{\partial t}+u_{j} \frac{\partial c}{\partial x_{j}}=D \frac{\partial^{2} c}{\partial x_{j} \partial x_{j}}
$$

where $D=10^{-9} \mathrm{~m}^{2} \mathrm{~s}^{-1}[50]$, equal to the diffusivity coefficient of amino-acid derived odorants in water. The full Navier-Stokes equation was solved for velocity, pressure and odorant concentration using the $\mathrm{CFX}^{\mathrm{TM}}$ solver on a mesh of $3.3 \times 10^{6}$ nodes. For eddies smaller in size than the grid size, a filter was applied using: $u=\bar{u}+u^{\prime}$, where $\bar{u}$ is the filtered velocity and $u^{\prime}$ is the residual velocity. The governing equation for the filtered velocity $u$ and scalar variable $c$ are given by:

$$
\begin{aligned}
\frac{\partial \bar{u}_{i}}{\partial t}+\frac{\partial}{\partial x_{j}}\left(\bar{u}_{i} \bar{u}_{j}\right) & =-\frac{1}{\rho} \frac{\partial \bar{p}}{\partial x_{i}}+2 \frac{\partial}{\partial x_{j}}\left[\left(\nu+\nu_{R}\right) \bar{S}_{i j}\right] \\
\frac{\partial \bar{c}}{\partial t}+\frac{\partial}{\partial x_{j}}\left(\bar{u}_{j} \bar{c}\right) & =\frac{\partial}{\partial x_{j}}\left[\left(D+D_{R}\right) \frac{\partial c}{\partial x_{j}}\right]
\end{aligned}
$$

The eddy viscosity is modeled as $\nu_{R}=\left(C_{s} \Delta_{g}\right)^{2} \sqrt{2 \bar{S}_{i j} \bar{S}_{i j}}$, where $\bar{S}_{i j}=\frac{1}{2}\left(\frac{\partial \bar{u}_{i}}{\partial x_{j}}+\frac{\partial \bar{u}_{j}}{\partial x_{i}}\right)$ is the 
strain-rate tensor and $\Delta_{g}$ is the grid size. The eddy diffusivity is $D_{R}=\nu_{R} / S c$. Two velocities were studied, with a uniform inlet speed of $U_{0}=10$ and $15 \mathrm{~cm} \mathrm{~s}^{-1}$, which corresponds to Kolmogorov scales of $\eta=1.3$ and $1.0 \mathrm{~mm}$ and Batchelor scales of $\lambda_{B}=0.03$ and $0.02 \mathrm{~mm}$ respectively. The boundary conditions for the model were:

- Inlet: water entered at a uniform speed of $U_{0}\left(=10\right.$ and $\left.15 \mathrm{~cm} \mathrm{~s}^{-1}\right)$. The odorant stream entered the flow domain $3 \mathrm{~mm}$ above the bed through a circular opening $(2 \mathrm{~mm}$ diameter) at a speed of $U_{0}$ and a concentration of $c_{\text {source }}=100 \mu \mathrm{g} \mathrm{cm}^{-3}$.

- Outlet: water and odorants exited the flow domain at a fixed outlet pressure of $\mathrm{p}=1$ atm.

- Sides and top: the normal components of the velocity and scalar variable gradients were set to zero: $\partial u / \partial n=0, \partial c / \partial n=0$, where $\mathrm{n}$ is normal direction to the corresponding boundary.

- Bottom: no-slip wall boundary condition was applied at the bottom boundary: $u_{x}=$ $u_{y}=u_{z}=0$

\subsubsection{Mesh convergence}

The outlet flow profile at $y=0$ (central plane) through the height of the column was compared at different mesh sizes. Figure 3.4Outlet flow velocity profile over the height of the flume column for different mesh resolutions. A mesh size of 3.3 million elements is used for the flume model.figure.caption.34 shows that the outlet flow profile does not change by any appreciable amount among the mesh sizes of 2.7, 3.3, 3.7 and 4.6 million elements. The mesh size of 3.3 million elements was used for the model since it allows a grid size of $\sim 0.1 \mathrm{~mm}$ near the roughness structures which is close to the Kolmogorov scale for this flow, hence allowing for the resolution of all eddies larger than this size and the sub-grid model is used for only the eddies smaller in size. 


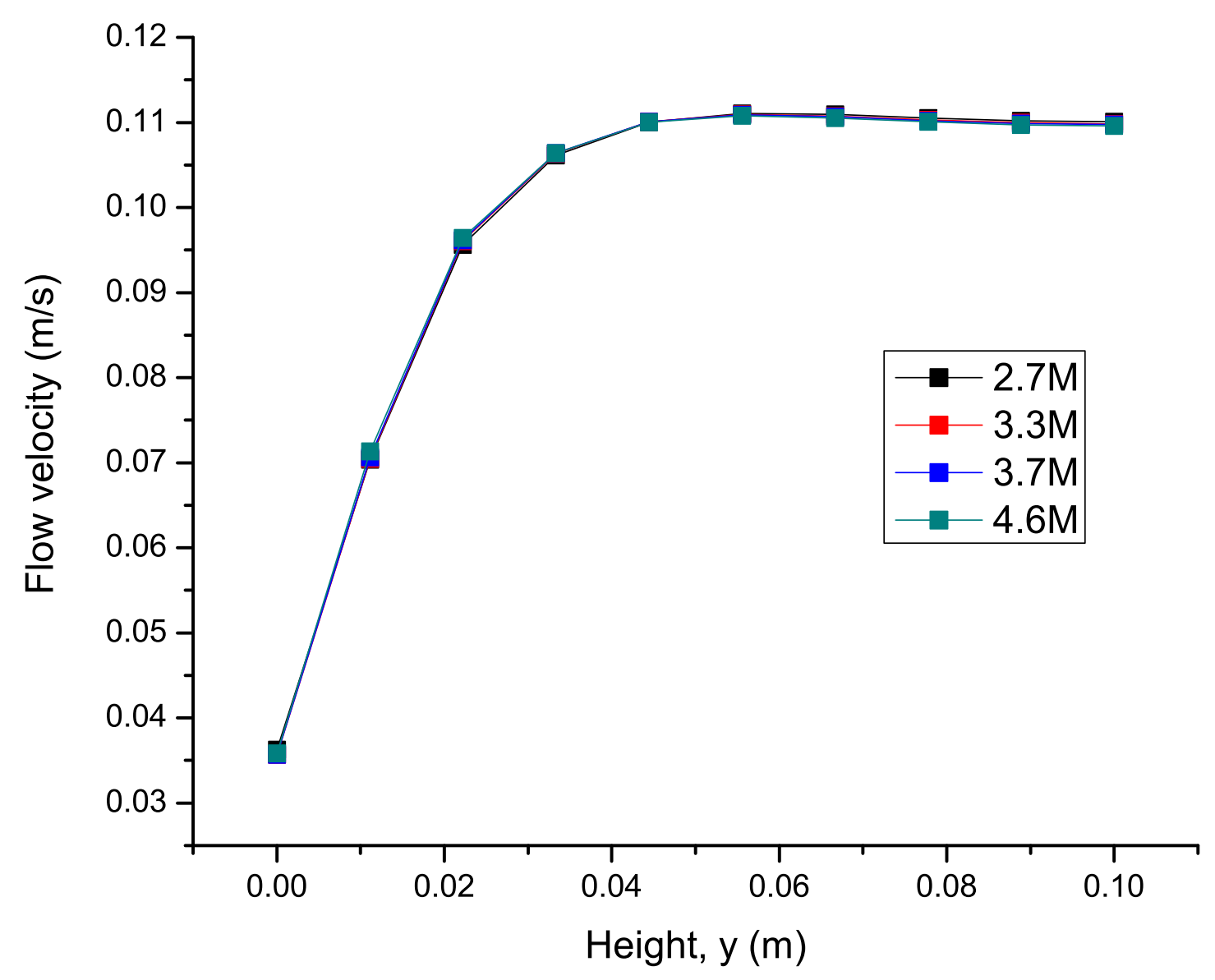

Figure 3.4: Outlet flow velocity profile over the height of the flume column for different mesh resolutions. A mesh size of 3.3 million elements is used for the flume model.

\subsection{Results}

\subsubsection{Laboratory Flume Studies}

Instantaneous particle motions from PIV and dye concentrations from PLIF are shown in Figure 3.5(A) Image of particle-laden fluid used in the PIV analysis; (B) PLIF image of plume structure $1 \mathrm{~m}$ downstream of the source; (C) combined plume structure from PLIF and velocity vectors (shown as blue arrows) calculated from PIV cross-correlation analysis. Odorant was released $1 \mathrm{~m}$ upstream from the imaging area by emitting fluorescein dye from 
a $1 \mathrm{~cm}$ tygon tube embedded across the width of the flume in the sandy bed material. Flow is from left to right.figure.caption.35A,B respectively. Velocity vectors, computed from PIV analysis, were superimposed over the PLIF image to quantify simultaneous water velocities and odorant concentrations $1 \mathrm{~m}$ downstream from the source, along the plume centerline (Figure 3.5(A) Image of particle-laden fluid used in the PIV analysis; (B) PLIF image of plume structure $1 \mathrm{~m}$ downstream of the source; (C) combined plume structure from PLIF and velocity vectors (shown as blue arrows) calculated from PIV cross-correlation analysis. Odorant was released $1 \mathrm{~m}$ upstream from the imaging area by emitting fluorescein dye from a $1 \mathrm{~cm}$ tygon tube embedded across the width of the flume in the sandy bed material. Flow is from left to right.figure.caption.35C). PLIF images show the highly filamentous structure and inherent intermittency in the turbulent odorant signal. From these PIV and PLIF images, instantaneous velocities and concentrations were separated into time-averaged and turbulent fluctuations of horizontal velocity, $u=\bar{u}+u^{\prime}$, vertical velocity, $w=\bar{w}+w^{\prime}$, and concentration of $c=\bar{c}+c^{\prime}$. The instantaneous horizontal turbulent flux of odorants was then computed as $u^{\prime} c^{\prime}$, as well as the time-averaged mean flux as $u^{\top} c^{\prime}$. Fluxes were computed on the spatial scale of the velocity, after concentration was averaged over the 8 pixel by 8 pixel PIV subwindow. Utilizing 5,000 PIV and 5,000 PLIF images (a total sampling time of $200 \mathrm{~s}$ ) for each flow condition, the temporal average of the horizontal velocity, normalized odorant concentration, and horizontal flux of odorants $\left(u^{\prime} c^{\prime}\right)$ was computed (Figure 3.6(A) Mean and fluctuating components of horizontal velocity within the laboratory flume from PIV. Fluctuating component computed as the root mean square (rms) of the horizontal velocity; (B) Mean and rms fluctuating component of normalized concentration from PLIF; (C) Mean horizontal turbulent flux $\left(\mathrm{g} \mathrm{cm}^{-2} \mathrm{~s}^{-1}\right)$, computed as mean of the fluctuating horizontal velocity, $u^{\prime}$, (in $\mathrm{cm} \mathrm{s}^{-1}$ ), multiplied by the fluctuating normalized concentration, $c^{\prime}$ figure.caption.36). The instantaneous measurements of velocity, concentrations, and fluxes encountered by animals navigating in the plume show substantially higher variability than time-averaged values. Since the temporal mean of fluctuations, $\bar{u}^{\prime}$ and $\overline{u^{\prime}}$ is by definition equal to 0 , the 
magnitude of these fluctuations was instead computed as the root mean square (rms) of $u$ and $c$ (Figure 3.6(A) Mean and fluctuating components of horizontal velocity within the laboratory flume from PIV. Fluctuating component computed as the root mean square (rms) of the horizontal velocity; (B) Mean and rms fluctuating component of normalized concentration from PLIF; (C) Mean horizontal turbulent flux $\left(\mathrm{g} \mathrm{cm}^{-2} \mathrm{~s}^{-1}\right)$, computed as mean of the fluctuating horizontal velocity, $u^{\prime}$, (in $\mathrm{cm} \mathrm{s}^{-1}$ ), multiplied by the fluctuating normalized concentration, $c^{\prime}$ figure.caption.36A,B respectively). This indicates that the magnitude of the horizontal velocity fluctuations were relatively constant throughout the water column, but due to a logarithmic distribution with height of the mean velocity profile, $u^{\prime}$ varied from approximately $45 \%$ of $\bar{u}$ near the bed $(z=0 \mathrm{~cm})$ and decreased to $20 \%$ of $\bar{u}$ at the elevation of antennules $(z=5 \mathrm{~cm}) . \bar{c}$ was highest adjacent to the sand bed and decreased with increased elevation, while the $c^{\prime}$ profile had a parabolic shape. Fluctuations in $c$ were approximately $20 \%$ of $\bar{c}$ near the bed, but increased to approximately $50 \%$ of $\bar{c}$ at $z=5 \mathrm{~cm}$. Turbulent fluxes, $u^{\top} c^{\prime}$, were relatively constant between $z=1 \mathrm{~cm}$ and $6 \mathrm{~cm}$, but decreased as the bed was approached $(z<1 \mathrm{~cm})$ due to enhanced mixing of the plume that decreased the magnitude in $c^{\prime}$. For $z>6 \mathrm{~cm}, u^{\prime} c^{\prime}$ decreased because the odorant plume had not mixed vertically to this elevation by the time the plume reached $1 \mathrm{~m}$ downstream from the source. Since the PIV and PLIF imaging occurred utilizing laser light aligned along a 2D plane, no transverse mixing of the plume $\left(u^{\prime} c^{\prime}\right)$ could be measured.

\subsubsection{Numerical Model}

A side and top-down view of odorant concentrations obtained from the 3D CFD model is shown in Figure 3.7(A) Side view of odorant concentration throughout the numerical flume. White squares are roughness elements along the bed; (B) Top-down view of odorant concentrations at an elevation of $z=0.5 \mathrm{~cm}$. The plume of odorants is released into the flume through a circular point-source inlet located at $z=0.3 \mathrm{~cm}$ above the bed.figure.caption.37. The turbulent stress, $u^{\prime} u^{\prime}$, through the water column shows close agreement between experimental 

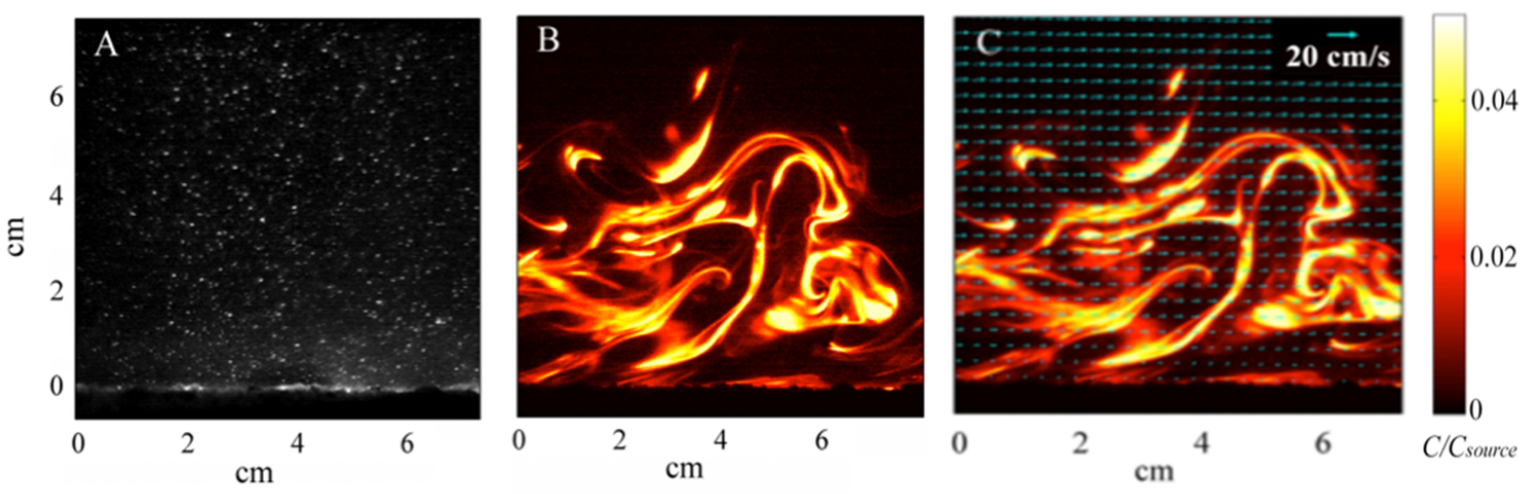

Figure 3.5: (A) Image of particle-laden fluid used in the PIV analysis; (B) PLIF image of plume structure $1 \mathrm{~m}$ downstream of the source; (C) combined plume structure from PLIF and velocity vectors (shown as blue arrows) calculated from PIV cross-correlation analysis. Odorant was released $1 \mathrm{~m}$ upstream from the imaging area by emitting fluorescein dye from a $1 \mathrm{~cm}$ tygon tube embedded across the width of the flume in the sandy bed material. Flow is from left to right.
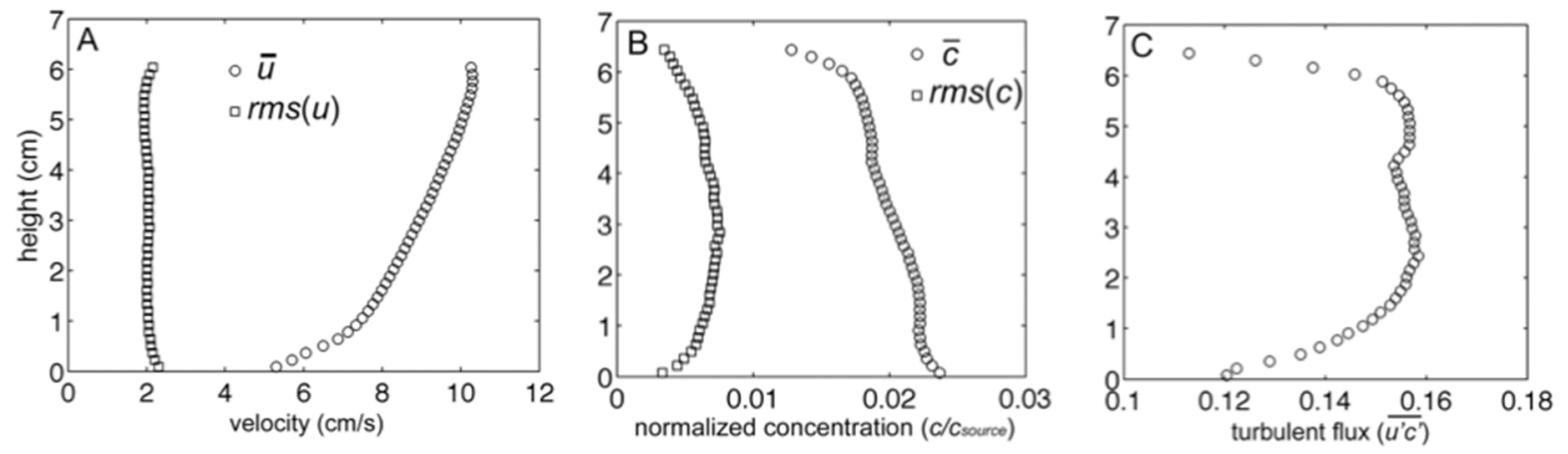

Figure 3.6: (A) Mean and fluctuating components of horizontal velocity within the laboratory flume from PIV. Fluctuating component computed as the root mean square (rms) of the horizontal velocity; (B) Mean and rms fluctuating component of normalized concentration from PLIF; (C) Mean horizontal turbulent flux $\left(\mathrm{g} \mathrm{cm}^{-2} \mathrm{~s}^{-1}\right)$, computed as mean of the fluctuating horizontal velocity, $u^{\prime}$, (in $\mathrm{cm} \mathrm{s}^{-1}$ ), multiplied by the fluctuating normalized concentration, $c^{\prime}$

and simulated model data (Figure 3.8Comparison of horizontal turbulence intensity within the flume experiments and numerical model. Measurements were obtained at $1 \mathrm{~m}$ downstream from the source.figure.caption.38A), except for the region adjacent to the bed, where the numerical roughness elements create a notable shear in the flow and a localized increase in 
$u^{\bar{\prime}} u^{\prime}$. The CFD model aimed to create a turbulent flow structure similar to that measured for the flume studies. Although the sand bed topography cannot be exactly reproduced within a numerical simulation, and thus some deviation of the turbulence structure and thus dispersal characteristics of odors between lab and numerical studies will exist, a benefit of the CFD model is that all three components of velocity, as well as concentration, can be computed at all locations simultaneously within the plume. Smaller odorant concentrations are predicted by the CFD model ( $1 \%$ of $c_{\text {source}}$, Figure 3.7(A) Side view of odorant concentration throughout the numerical flume. White squares are roughness elements along the bed; (B) Top-down view of odorant concentrations at an elevation of $z=0.5 \mathrm{~cm}$. The plume of odorants is released into the flume through a circular point-source inlet located at $z=0.3 \mathrm{~cm}$ above the bed.figure.caption.37) compared to the experimental flume data (up to $3 \%$ of $c_{\text {source }}$, Figure 3.5(A) Image of particle-laden fluid used in the PIV analysis; (B) PLIF image of plume structure $1 \mathrm{~m}$ downstream of the source; (C) combined plume structure from PLIF and velocity vectors (shown as blue arrows) calculated from PIV cross-correlation analysis. Odorant was released $1 \mathrm{~m}$ upstream from the imaging area by emitting fluorescein dye from a $1 \mathrm{~cm}$ tygon tube embedded across the width of the flume in the sandy bed material. Flow is from left to right.figure.caption.35) because a point source of odorants was used in the CFD simulation, compared to a line source used for the flume experiments that released a greater amount of dye into the flow. Mean flow and turbulence characteristics for the two plumes generated in the CFD model (inlet flow conditions of $U_{0}=10$ and $15 \mathrm{~cm} \mathrm{~s}^{-1}$ ) are listed in Table 3.1Mean statistics for the simulated flow at inlet flow speeds of $U_{0}=10$ and $15 \mathrm{~cm}$ $\mathrm{s}^{-1}$ respectively. $U_{0}$ is the inlet velocity along the upstream end of the flow domain. $\bar{u}$ is the mean velocity over the bed roughness $1 \mathrm{~m}$ downstream from the source, $u_{*}$ is the friction velocity, $R e_{*}$ is the roughness Reynolds number computed as $R e_{*}=u_{*} h / \nu$ (h $=$ height of roughness element), $\eta$ is the Kolmogorov lengthscale, $\epsilon$ is the dissipation rate of turbulence and $\lambda_{B}$ is the Batchelor scale.table.caption.39. Instantaneous odorant concentration, velocity, and transverse flux, $v^{\prime} u^{\prime}$, along a horizontal plane $z=1 \mathrm{~cm}$ above the simulated flume bed 
are shown in Figure 3.9(A) Instantaneous (A) odorant concentration; (B) horizontal velocity; and (C) transverse concentration flux $\left(v^{\prime} c^{\prime}\right)$ at $z=1 \mathrm{~cm}$ above the flume bed, obtained at $t$ $=10 \mathrm{~s}$ after initial release of odorant.figure.caption.40. Due to the close proximity of the bed, both the odorant concentrations and velocity structure are significantly impacted by the cube-shaped bedforms, initially causing rapid dispersion of concentration at $x=0.05 \mathrm{~m}$ due to flow interaction with the first row of bedforms. This dispersion may partially be due to numerical error due to the steep change in roughness when flow first encounters the bedforms. However, this effect is localized and further downstream, the concentration structure evolves slowly, with higher concentrations near the source, and lower, more diffuse concentrations with greater downstream distance. The velocity structure shows uniform conditions along the upstream end of the model flume until the first bedforms occur. Further downstream, distinct turbulence structure is evident as water velocities are directly impacted by the bedforms. The transverse flux shows large variability, both in magnitude along the upstream-downstream, $x$, direction, and in sign (positive or negative) along the transverse, $y$, direction. At $z=1 \mathrm{~cm}$, the flux magnitude is greatest near the source, between $x=0.1$ and $0.4 \mathrm{~m}$ downstream, and is on average positive to the left of the plume centerline $(y>0 \mathrm{~cm})$, and negative to the right of the plume centerline $(y<0 \mathrm{~cm})$, if faced towards the downstream direction.

Table 3.1: Mean statistics for the simulated flow at inlet flow speeds of $U_{0}=10$ and $15 \mathrm{~cm}$ $\mathrm{s}^{-1}$ respectively. $U_{0}$ is the inlet velocity along the upstream end of the flow domain. $\bar{u}$ is the mean velocity over the bed roughness $1 \mathrm{~m}$ downstream from the source, $u_{*}$ is the friction velocity, $R e_{*}$ is the roughness Reynolds number computed as $R e_{*}=u_{*} h / \nu$ (h = height of roughness element), $\eta$ is the Kolmogorov lengthscale, $\epsilon$ is the dissipation rate of turbulence and $\lambda_{B}$ is the Batchelor scale.

\begin{tabular}{lllllll}
\hline & $u\left(\mathrm{~cm} \mathrm{~s}^{-1}\right)$ & $u_{*}\left(\mathrm{~cm} \mathrm{~s}^{-1}\right)$ & $R e_{*}$ & $\eta(\mathrm{cm})$ & $\epsilon\left(\mathrm{cm}^{2} \mathrm{~s}^{-3}\right)$ & $\lambda_{B}(\mathrm{~cm})$ \\
\hline Sand, $U_{0}=10 \mathrm{~cm} \mathrm{~s}^{-1}$ & 10.6 & 0.15 & 8.4 & 0.13 & $2.5 \times 10^{-3}$ & $2.9 \times 10^{-3}$ \\
Sand, $U_{0}=15 \mathrm{~cm} \mathrm{~s}^{-1}$ & 15.2 & 0.18 & 10.1 & 0.10 & $7.1 \times 10^{-3}$ & $2.2 \times 10^{-3}$ \\
\hline
\end{tabular}

At an elevation of $z=3 \mathrm{~cm}$ (Figure 3.10Instantaneous (A) odorant concentration; (B) horizontal velocity; and (C) transverse concentration flux $\left(v^{\prime} c^{\prime}\right)$ at $z=3 \mathrm{~cm}$ above the flume bed, obtained at $t=10 \mathrm{~s}$ after initial release of odorant.figure.caption.41), the influence 


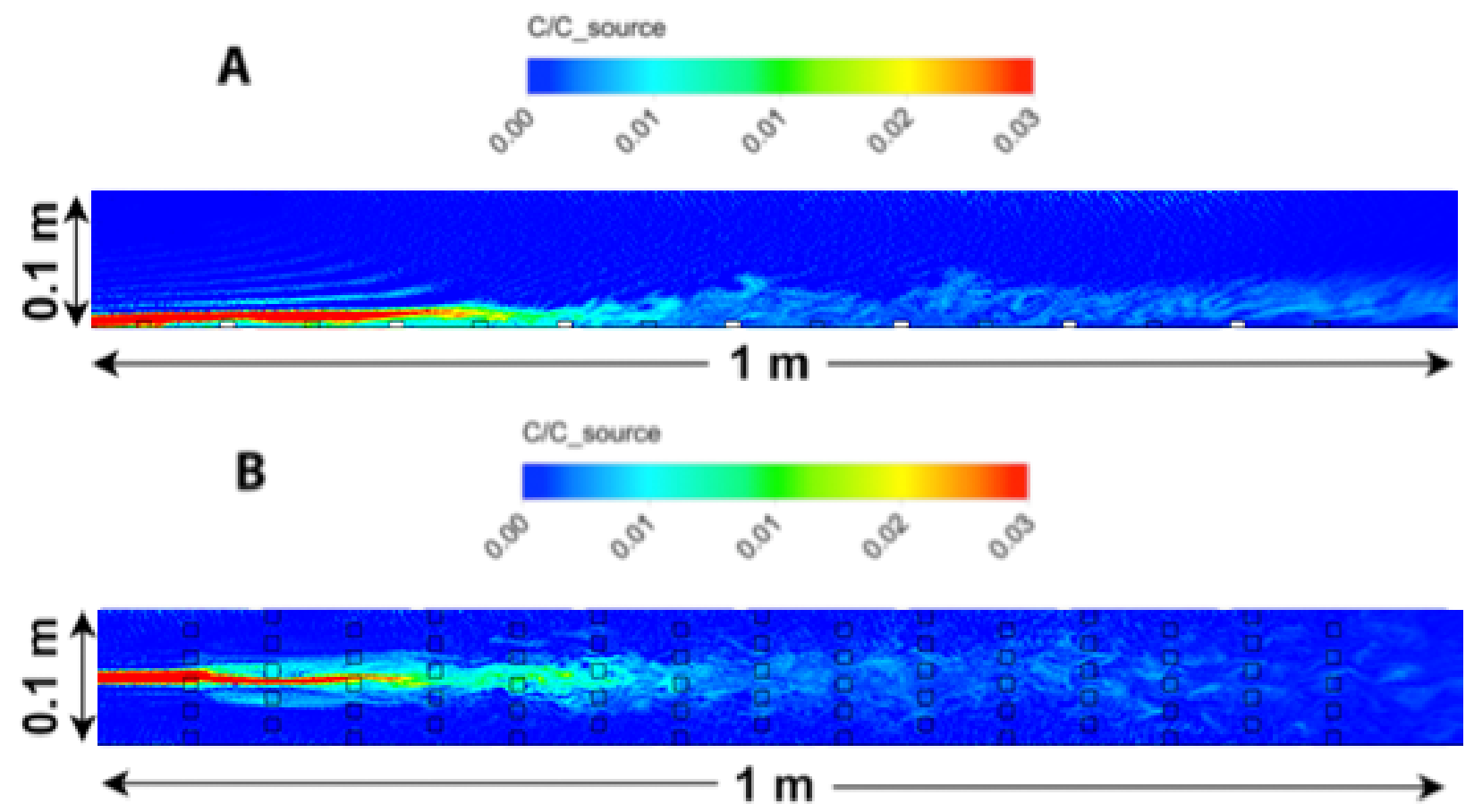

Figure 3.7: (A) Side view of odorant concentration throughout the numerical flume. White squares are roughness elements along the bed; (B) Top-down view of odorant concentrations at an elevation of $z=0.5 \mathrm{~cm}$. The plume of odorants is released into the flume through a circular point-source inlet located at $z=0.3 \mathrm{~cm}$ above the bed.

of turbulence generated at the bed is less evident, however, turbulent mixing still injects large fluctuations in both concentration and velocity. The intensity in the fluctuations changes along the upstream-downstream direction, and may provide information to organisms navigating in the plume. However laterally, neither the magnitude in concentration or velocity nor their intermittency give any indication of plume direction laterally across the centerline. Simultaneous measures of odorant and velocity fluctuations do show distinct signals of lateral direction of the plume, with negative fluxes to one side, and positive fluxes to the other side of the plume centerline. At $z=5 \mathrm{~cm}$ (Figure 3.11(A) Instantaneous (A) odorant concentration; (B) horizontal velocity; and (C) transverse concentration flux $\left(v^{\prime} c^{\prime}\right)$ at $z=5 \mathrm{~cm}$ above the flume bed, obtained at $t=10 \mathrm{~s}$ after initial release of odorant.figure.caption.42), the transverse flux increases in magnitude at greater downstream distances and spreads laterally compared to $z=1$ or $3 \mathrm{~cm}$. As is evident from Figures 3.9(A) Instantaneous (A) odorant 


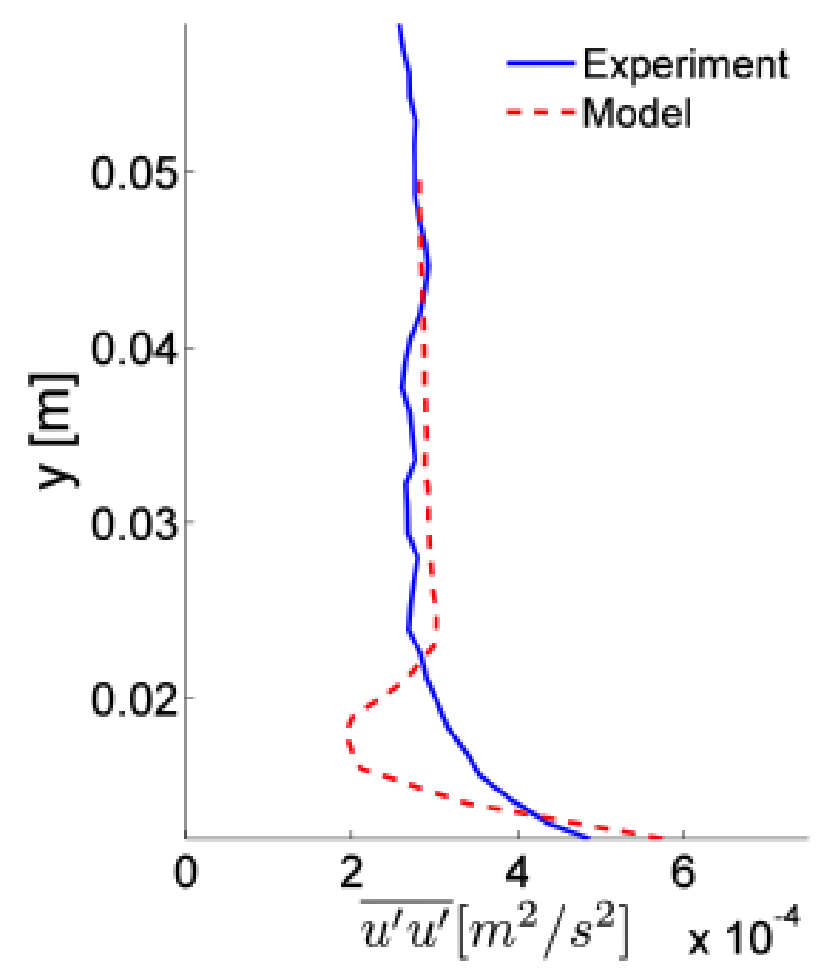

Figure 3.8: Comparison of horizontal turbulence intensity within the flume experiments and numerical model. Measurements were obtained at $1 \mathrm{~m}$ downstream from the source.

concentration; (B) horizontal velocity; and (C) transverse concentration flux $\left(v^{\prime} c^{\prime}\right)$ at $z=1$ $\mathrm{cm}$ above the flume bed, obtained at $t=10 \mathrm{~s}$ after initial release of odorant.figure.caption.403.11(A) Instantaneous (A) odorant concentration; (B) horizontal velocity; and (C) transverse concentration flux $\left(v^{\prime} c^{\prime}\right)$ at $z=5 \mathrm{~cm}$ above the flume bed, obtained at $t=10 \mathrm{~s}$ after initial release of odorant.figure.caption.42, as the source is approached the location of the largest magnitudes of transverse fluxes occur at a lower elevation and closer to the plume centerline, but remain on average negative fluxes for $y<0$ and positive fluxes for $y>0$. This suggests that changes in the magnitude and spread of the transverse flux can offer information about both the transverse and downstream distance from the source that neither the concentration nor ambient velocity can provide independently. 


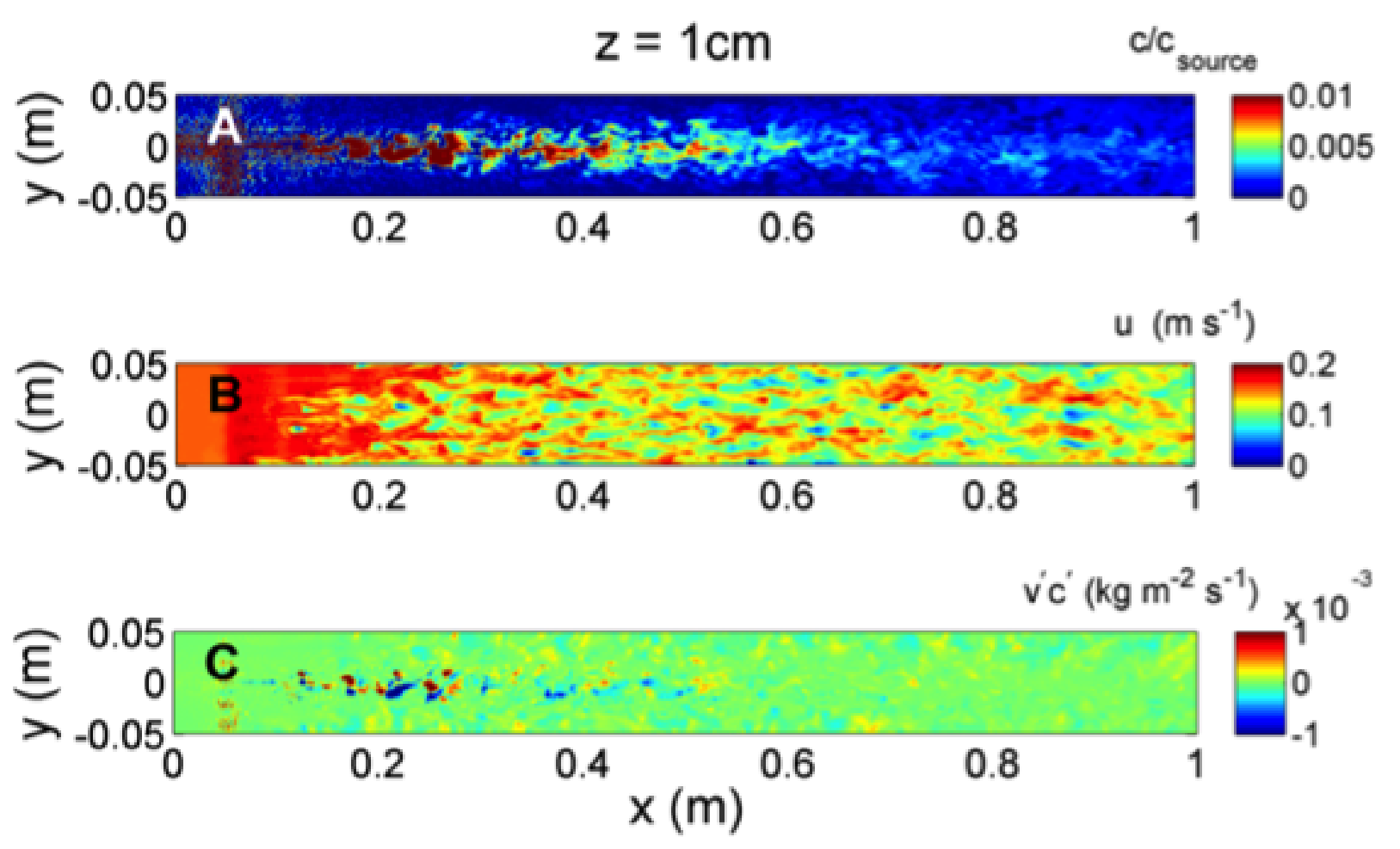

Figure 3.9: (A) Instantaneous (A) odorant concentration; (B) horizontal velocity; and (C) transverse concentration flux $\left(v^{\prime} c^{\prime}\right)$ at $z=1 \mathrm{~cm}$ above the flume bed, obtained at $t=10 \mathrm{~s}$ after initial release of odorant.

\subsubsection{Temporal and Spatial Averaging of the Plume}

The cumulative mean concentrations, velocities, and transverse fluxes sampled at two points and separated by $5 \mathrm{~cm}$ that span the centerline of the plume (at $y=2.5 \mathrm{~cm}$ and $-2.5 \mathrm{~cm}$ and a downstream location of $x=0.8 \mathrm{~m}$ ) are shown in Figure 3.12(A) Normalized concentration; (B) horizontal velocity, $u$; and (C) transverse flux $\left(v^{\prime} c^{\prime}\right)$ at points a and b (as indicated in Figure 3.11(A) Instantaneous (A) odorant concentration; (B) horizontal velocity; and (C) transverse concentration flux $\left(v^{\prime} c^{\prime}\right)$ at $z=5 \mathrm{~cm}$ above the flume bed, obtained at $t=10$ $\mathrm{s}$ after initial release of odorant.figure.caption.42C) for a $1 \mathrm{~s}$ time period. The solid lines show the cumulative mean and the shaded regions show the \pm 1 standard error around the cumulative mean.figure.caption.43. This is information that an organism, sampling at an elevation of $z=5 \mathrm{~cm}$ and utilizing two antennules spaced a distance of $5 \mathrm{~cm}$ (which is spacing observed for blue crab antennules [3]), could conceivably quantify if it remained stationary at 


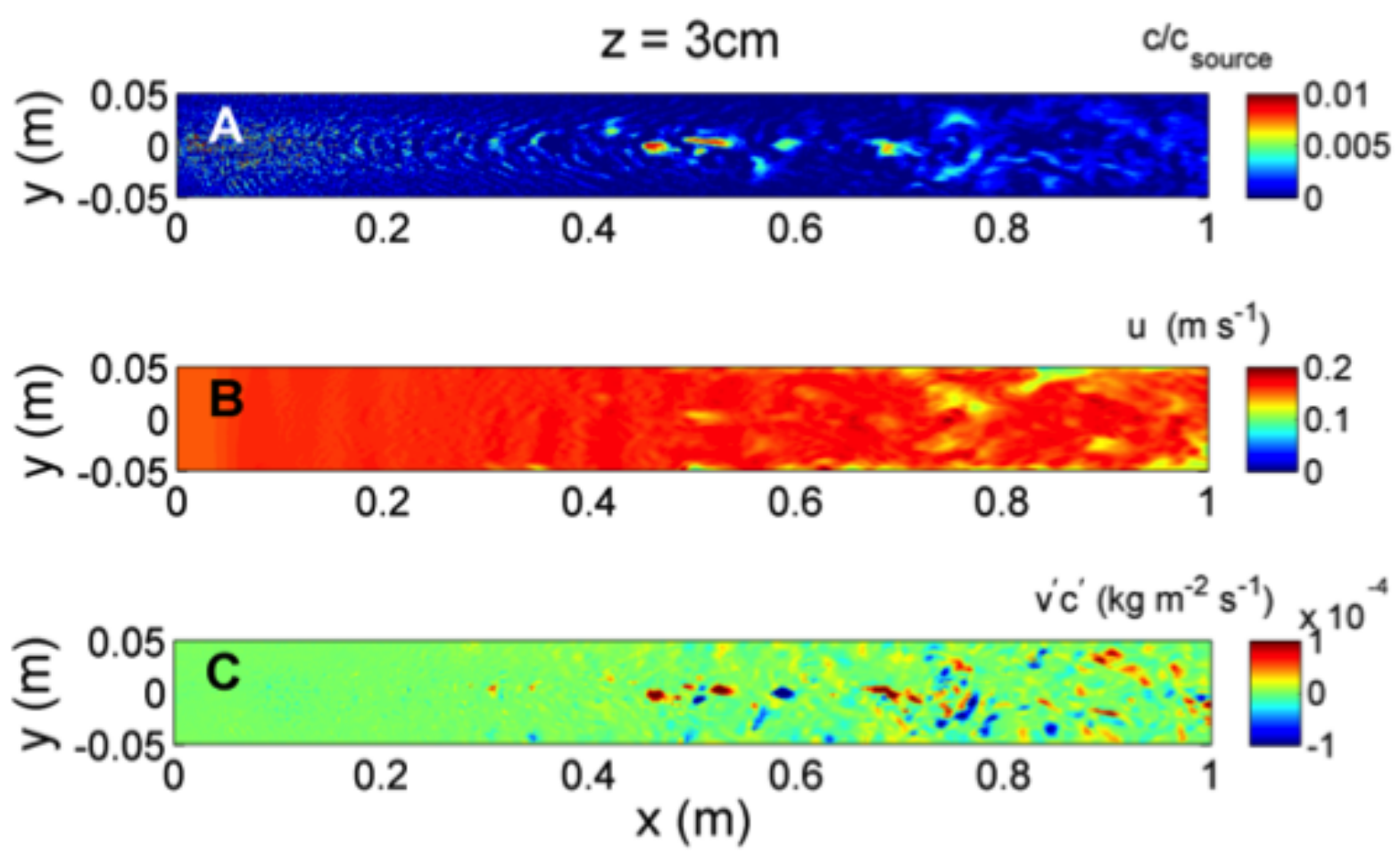

Figure 3.10: Instantaneous (A) odorant concentration; (B) horizontal velocity; and (C) transverse concentration flux $\left(v^{\prime} c^{\prime}\right)$ at $z=3 \mathrm{~cm}$ above the flume bed, obtained at $t=10 \mathrm{~s}$ after initial release of odorant.

this location. The cumulative mean converges, and the standard error becomes reduced the longer the organism samples at the same location. This suggests that little information can be statistically gained about the transverse location of the source from differencing values across the plume centerline if just concentration (Figure 3.12(A) Normalized concentration; (B) horizontal velocity, $u$; and (C) transverse flux $\left(v^{\prime} c^{\prime}\right)$ at points a and b (as indicated in Figure 3.11(A) Instantaneous (A) odorant concentration; (B) horizontal velocity; and (C) transverse concentration flux $\left(v^{\prime} c^{\prime}\right)$ at $z=5 \mathrm{~cm}$ above the flume bed, obtained at $t=10 \mathrm{~s}$ after initial release of odorant.figure.caption.42C) for a $1 \mathrm{~s}$ time period. The solid lines show the cumulative mean and the shaded regions show the \pm 1 standard error around the cumulative mean.figure.caption.43A) is quantified. Although variations in horizontal velocity (Figure 3.12(A) Normalized concentration; (B) horizontal velocity, $u$; and (C) transverse flux $\left(v^{\prime} c^{\prime}\right)$ at points a and b (as indicated in Figure 3.11(A) Instantaneous (A) odorant concentration; 


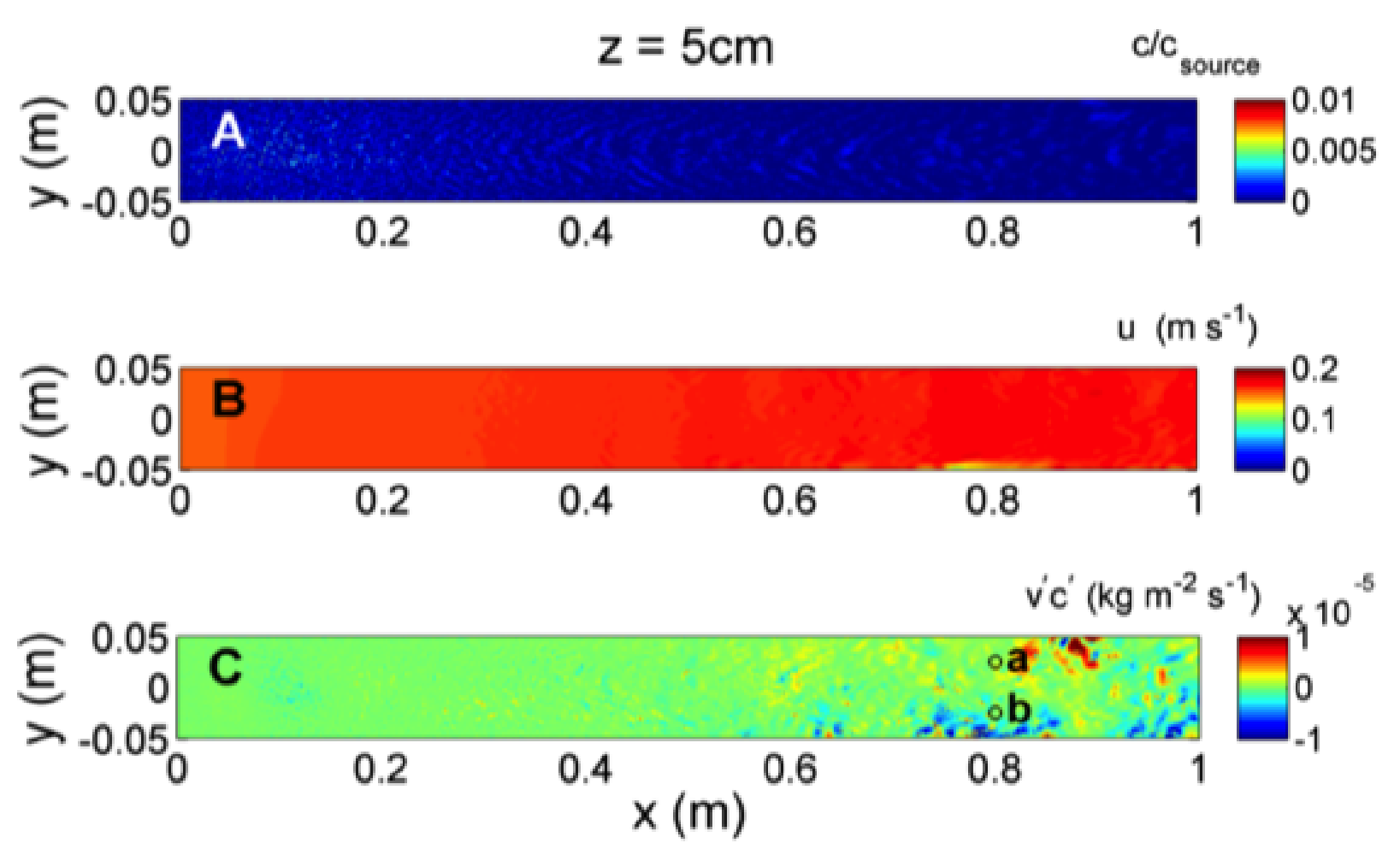

Figure 3.11: (A) Instantaneous (A) odorant concentration; (B) horizontal velocity; and (C) transverse concentration flux $\left(v^{\prime} c^{\prime}\right)$ at $z=5 \mathrm{~cm}$ above the flume bed, obtained at $t=10 \mathrm{~s}$ after initial release of odorant.

(B) horizontal velocity; and (C) transverse concentration flux $\left(v^{\prime} c^{\prime}\right)$ at $z=5 \mathrm{~cm}$ above the flume bed, obtained at $t=10 \mathrm{~s}$ after initial release of odorant.figure.caption.42C) for a $1 \mathrm{~s}$ time period. The solid lines show the cumulative mean and the shaded regions show the \pm 1 standard error around the cumulative mean.figure.caption.43B) do occur at the two locations due to turbulent eddies, no statistical difference in velocity occurs after averaging for periods longer than $0.7 \mathrm{~s}$. However, statistically significant differences in transverse flux can be quantified even after sampling for as little as $200 \mathrm{~ms}$ at this location, and these differences remain statistically significant for time periods $>1 \mathrm{~s}$. Although the length of time needed to determine statistically different transverse fluxes is dependent upon the location within the plume and initial concentration measured, typically the fluxes show statistical distinct values in $<0.5 \mathrm{~s}$, while velocity and concentration tend to statistically converge over a similar length of time. The time-averaged concentration field as a function of downstream distance along 
the plume centerline is shown in Figure 3.13(A) Normalized concentration and (B) temporal rate of change of concentration $(d c / d t)$ along the centerline through the length of the flume at $z=5 \mathrm{~cm}$. The solid line shows mean values and the shaded region shows the region of \pm 1 standard deviation around the mean.figure.caption.44A for both the $U_{0}=10 \mathrm{~cm} \mathrm{~s}^{-1}$ and 15 $\mathrm{cm} \mathrm{s}^{-1}$ mean flow scenarios. Mean values of $c / c_{\text {source }}$ (solid red and blue lines) increase slowly as the source is approached, but do not change appreciably due to changes in the mean flow. However, larger fluctuations in concentration occur as the source is approached, indicating that the plume becomes more intermittent. Figure 3.13(A) Normalized concentration and (B) temporal rate of change of concentration $(d c / d t)$ along the centerline through the length of the flume at $z=5 \mathrm{~cm}$. The solid line shows mean values and the shaded region shows the region of \pm 1 standard deviation around the mean.figure.caption.44B shows the concentration slope, computed as the temporal rate of change of concentration $(d c / d t)$. For both flow conditions, larger fluctuations in $d c / d t$ also occur as the source is approached. However, mean and fluctuating estimates in both $c / c_{\text {source }}$ and $d c / d t$ evolve slowly with downstream distance, and either long sampling times, or large movements in upstream-downstream distance are necessary to statistically see changes in plume dynamics that might aid in locating the source.

\subsection{Discussion}

Results indicate that simultaneous detection of flow and odorants within a turbulent plume can aid aquatic organisms in tracking a source. To develop this link between signal detection, potential tracking behavior and odorant signal properties, we utilized flume experiments that simultaneously quantified spatial variability in both velocity and concentration over a bed composed of sand. We utilized results from the laboratory experiments to develop a 3-dimensional computational fluid dynamics model of a similar turbulent plume, and developed the model for two flow conditions, with inlet velocities $U_{0}=10 \mathrm{~cm} \mathrm{~s}^{-1}$ and $15 \mathrm{~cm}$ $\mathrm{s}^{-1}$. Our results, as well as results from other studies [8, 42, 88], suggest that time-averaged 

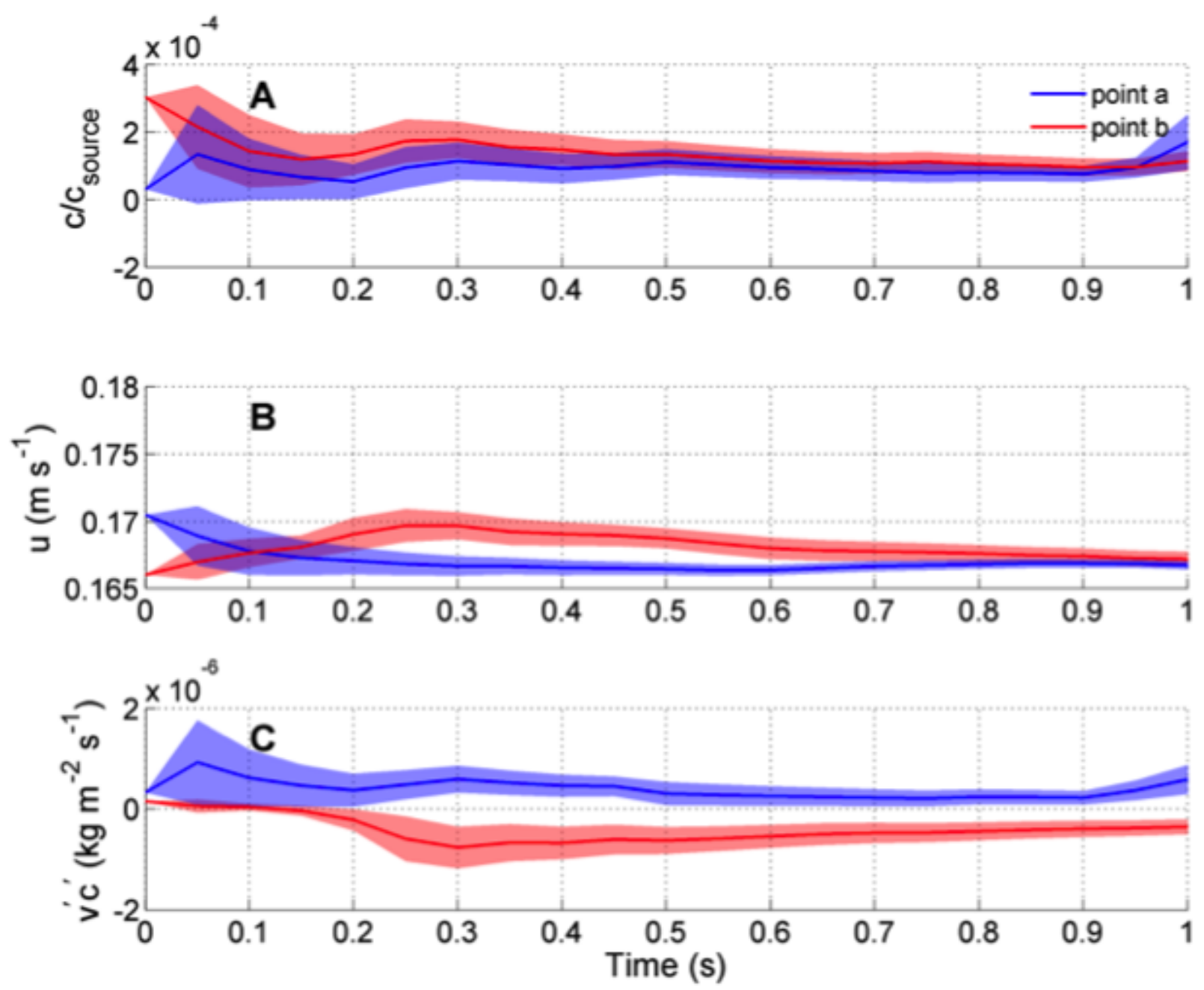

Figure 3.12: (A) Normalized concentration; (B) horizontal velocity, $u$; and (C) transverse flux $\left(v^{\prime} c^{\prime}\right)$ at points a and b (as indicated in Figure 3.11(A) Instantaneous (A) odorant concentration; (B) horizontal velocity; and (C) transverse concentration flux $\left(v^{\prime} c^{\prime}\right)$ at $z=5$ $\mathrm{cm}$ above the flume bed, obtained at $t=10 \mathrm{~s}$ after initial release of odorant.figure.caption.42C) for a $1 \mathrm{~s}$ time period. The solid lines show the cumulative mean and the shaded regions show the \pm 1 standard error around the cumulative mean.

concentration as well as the slope, computed as the concentration gradient with respect to time $(d c / d t)$, converge slowly, and the length of sampling period by an animal too great, to be effectively used in determining position in a plume relative to the source. However, instantaneous fluctuations in concentration, in combination with transverse variations in velocity, vary on sufficiently short timescales to be effectively used to determine directional location of the plume source, potentially by a variety of aquatic organisms [80, 35, 89]. 

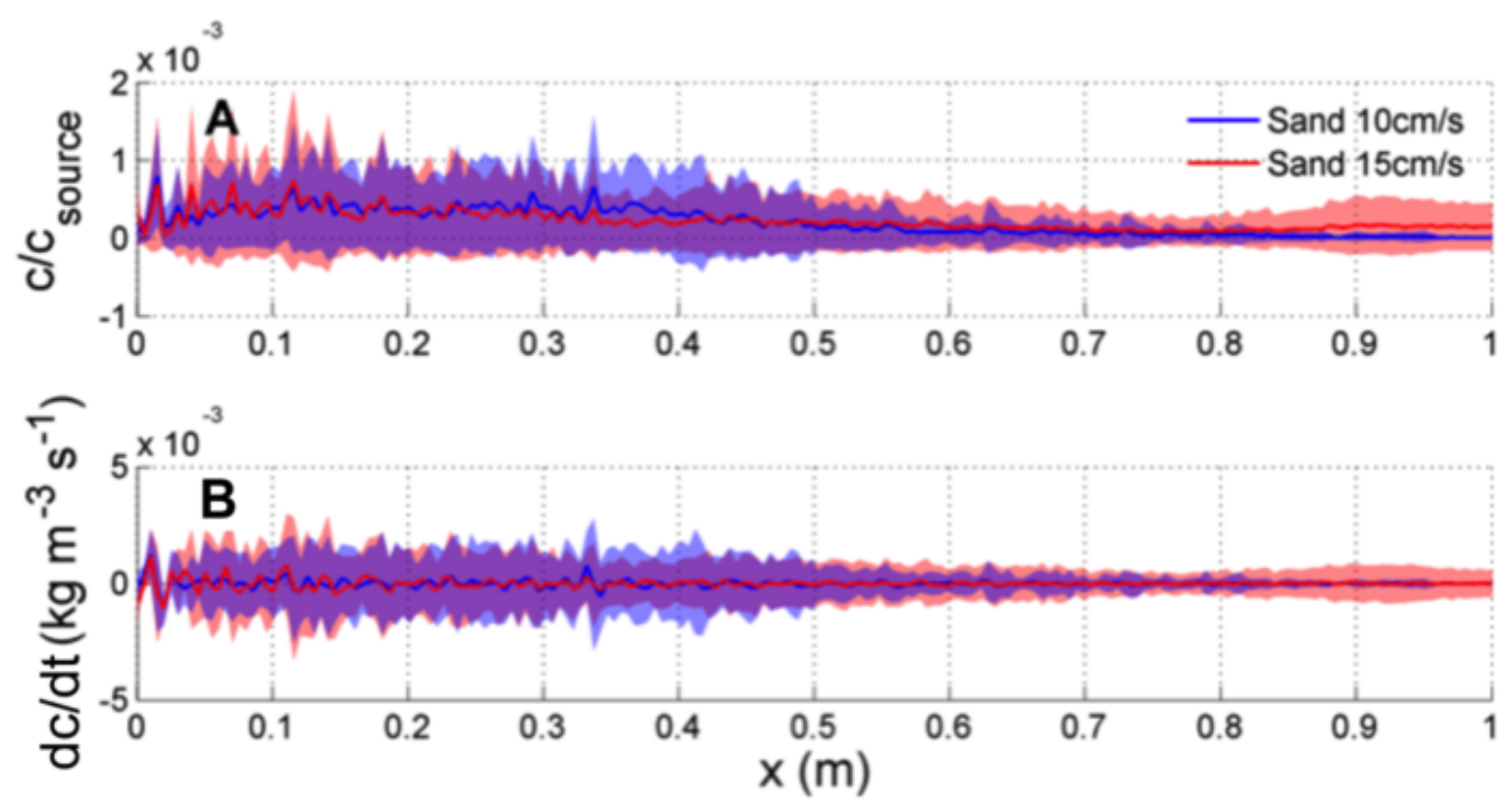

Figure 3.13: (A) Normalized concentration and (B) temporal rate of change of concentration $(d c / d t)$ along the centerline through the length of the flume at $z=5 \mathrm{~cm}$. The solid line shows mean values and the shaded region shows the region of \pm 1 standard deviation around the mean.

Specifically, the transverse flux of odorants, $v^{\prime} c^{\prime}$, vary both in magnitude and sign, depending on whether the flux is sampled to the left or right of the centerline of the plume. In addition, the transverse difference in magnitude and sign of the flux converge towards the centerline of the plume, and at lower elevations above the bed, as the source is approached. These dynamics suggest that animals, if tracking the edge of the plume where transverse fluxes are largest, can utilize variations in flux magnitude to aid in determining both the lateral location, and downstream distance from the source. Since the sign of the flux also changes depending upon the left or right hand side of the plume relative to the centerline, bilateral comparisons utilizing two antennules are not needed to determine correct orientation within a plume. This agrees with previous findings that the use of only one antennule is sufficient for chemo-orientation within a plume [90], although the use of bilateral comparisons have been shown to enhance the effectiveness and speed of the search [38]. There are many reported search strategies that organisms employ to undergo search, including odor gated rheotaxis where an animal moves upstream as long as sufficient contact with odor is maintained, or 
eddy chemo-rheotaxis where an animal steers towards a source using eddies containing higher concentrations of odorant (reviewed in $[8,35]$ ). However, many organisms, including crabs and some lobster species, appear to track lateral edges of the plume (i.e., plume edge tracking [35]) and move towards the centerline as the source is approached [80, 89] thus moving along a direction indicating that transverse flux information might be utilized. Although integration of flow and concentration cues on timescales between 0.1 and $1 \mathrm{~s}$ have been shown in sharks, where both sensing along their lateral line [76] and bilateral odor concentration differences between their nares [91] altered orientation behavior to dispersing odorants, no studies to date have directly linked the ability of crustaceans to integrate flow and odorant information to aid in search on these short timescales. Dickman et al. [92] utilized measurements of an instantaneous 3D concentration field surrounding actively tracking blue crabs and found a connection between upstream walking speed and bursts of odorant concentration arriving at the antennule chemosensors. The antennule elevation of the crabs generally changed as crabs lowered or raised their thorax as they moved upstream, while transverse crab movements were in apparent response to the transverse distribution of the odorant concentration field. This study also suggested that asymmetry in the odorant concentration distribution at the elevation of the leg chemosensors (at approximately $z=1 \mathrm{~cm}$ ) was responsible for position adjustments in the transverse direction. Although cross-stream motion is aided by chemical signal inputs to receptors on their walking legs, crabs do make rotational movements in response to chemical signals along their antennules, which determine the crabs body angle with respect to the flow [72].

\subsubsection{Neural Responses to Odorants and Flows}

Reidenbach and Koehl [14] found that the intermittency in encounter rates of odors is greater when an odor plume is sampled by flicking than that for continuous sampling, giving receptor

neurons more time to dis-adapt before encountering the next odor pulse. Adaptation of receptor neurons occurs due to prolonged exposure to odorants, and subsequent responses 
to odor pulses is reduced or absent [93, 47]. Flicking therefore enables sensory neurons to remain sensitive to intermittent changes in odor concentration $[61,65]$. For the clawed lobster Homarus americanus, receptor neurons in the antennule respond, after a latency period of at least $50 \mathrm{~ms}$, with a series of action potential spikes. However, after exposure to odorants for $300 \mathrm{~ms}$, spike frequency is reduced, and neurons cease firing after 1,500 ms [62, 66]. This adaptation resets the sensitivity of the neurons so that they can respond to transient changes in odor concentration relative to background concentrations. This suggests that lobsters need to be exposed to odorants for at least $50 \mathrm{~ms}$ for neurons to respond, but for exposure times greater than 1,500 ms, neurons cease responding to ambient background concentrations. Although intermittency in the odorant concentrations is essential for accurate detection of ambient concentrations, adaptation may be beneficial to allow organisms to respond to concentration changes relative to background. While adaptation to odor exposure is relatively rapid, disadaptation in individual H. americanus lobster ORNs averaged $14 \mathrm{~s}$ [61]. Longer exposure to background concentrations generated more ORN spikes, but did not increase the initial spike frequency. These findings suggest that certain ORNs, at least in $\mathrm{H}$. americanus, are sensitive to changes in the ratio of stimulus concentration to background concentration, and allow the receptors to function with equal sensitivity across at least three orders of magnitude in background concentration [65]. This essentially allows these organisms to respond to changes in concentrations relative to background levels [94]. These relative changes in concentration and velocity relative to mean background conditions are also utilized to determine mathematically the magnitude of instantaneous flux events. This may also suggest why many aquatic organisms are found to track the edges of the plume, where both the transverse flux is largest, and intermittency is increased [14]. In addition, statistical differences in the magnitude of the transverse flux can be obtained on timescales of $\leq 1 \mathrm{~s}$, which is within the range of neural response times of aquatic animals. 


\subsection{Conclusions}

This study shows that simultaneous sampling of both flow and odorant concentration can provide valuable cues for tracking of odorant plumes compared to sampling of odorants alone. Mean odorant concentration increases as the animal moves towards the plume source, helping it determine the upstream direction. Variability in the temporal rate of change of concentration also increases as the animal moves towards the source. However, both these statistics converge slowly, and therefore may not be useful for aquatic organisms that often make rapid movements while navigating in a plume [8]. Comparison of flux of odorants in the transverse direction between two points on either side of the plume centerline separates statistically on timescales $\leq 0.5 \mathrm{~s}$, while velocity and concentration tend to converge over similar lengths of time. This difference in flux becomes larger, and converges towards the plume centerline as the animal moves closer to the plume source. Although it is still unknown if crustaceans can integrate variations in odorant concentrations and velocity relative to background levels, our results suggest that flux can be a significant factor enabling animals to orient themselves favorably in the search for a plume source, and occurs on timescales relevant to responses by olfactory neurons. In addition, our results show that transverse flux is a useful parameter to be quantified and incorporated into the design and utilization of artificial bio-mimetic sensors. 


\section{CHAPTER 4}

\section{Effects of sensilla morphology on}

\section{mechanosensory sensitivity in}

\section{crustaceans}

\section{Abstract}

Within aquatic environments, crustaceans use hydrodynamic and contact sensilla to detect the presence of predators and prey, and the fluid motion they create [11,6]. Combined with chemoreceptors, these mechano-receptors also aid animals in the tracking of odorant sources. The morphologies of these sensilla vary widely, and presumably are adapted to perform specific sensory functions across a range of sensitivities. Along the antennule of the freshwater crayfish, Procambarus clarkii, four predominant mechanosensory sensilla morphologies are found. To study their response to upstream flow perturbations, atomic force microscopy was utilized to determine sensilla bending in response to an applied force. A Numerical model was then developed and the four sensilla morphologies were simulated to quantify their deformation due to an applied force caused by flow perturbations within their surrounding fluid. These flow perturbations were intended to mimic predator and ambient 
fluid movements. The four sensilla include the feathered and filamentous sensilla, which are hydrodynamic receptors, and the beaked and asymmetric sensilla, which are bimodal hydrodynamic and chemical receptors. Results show that the upstream fluid motion causes alterations in velocity near the sensilla accompanied by corresponding variations in pressure. The standing feathered sensilla experience the greatest fluid force and bending moments, while the filamentous sensilla experience maximum angular deformation. The sensitivity of the sensilla correlates closely to their sensory function. The feathered and filamentous hydrodynamic sensilla, which likely respond to predator movement, were found to be highly sensitive to flow perturbations. The beaked and asymmetric sensilla, which are bimodal chemo-mechanoreceptors that likely function under faster antennule flicking movements, were found to be much less sensitive. To study the directional dependence of sensilla sensitivity, numerical simulation were performed to quantify the deformation of sensilla as a function of the direction of hydrodynamic perturbation relative to the sensilla orientation. Results show similar sensitivity at all angles except at $180^{\circ}$ where a sharp drop in sensitivity in observed. This morphological sensitivity to directional fluid perturbations agrees well with neural responses measured from within the crayfish brain.

\subsection{Introduction}

Arthropod crustaceans such as crabs, lobsters and crayfish use contact, hydrodynamic, and chemical sensing for a number of activities such as detecting and locating predators, prey, habitat and potential mates $[11,6,7]$. These organisms inhabit benthic environments, and are often exposed to moving fluids that contain both hydrodynamic and chemical cues. The benthic zone is often highly turbulent, and therefore these organisms are likely to be surrounded by intense gradients in both water velocities and chemical concentrations $[14,95]$, which can provide information, but also uncertainty, when detecting and tracking

movements and distant odor sources [8]. These organisms contain unimodal chemosensitive 
and hydrodynamically sensitive setae, along with biomadal chemo-mechanosensitive setae that respond to both flows and odors $[46,7]$. These setae can be found along their first antennae, called antennules, in addition to sensilla that are often present over large portions of their body $[75,32]$.

Hydrodynamic stimulation of the antennules, including flicking and from ambient current evokes electrical activity in the central brain neurons [7]. In addition to chemosensory aesthetascs, there is a rich diversity of sensilla located on antennules of crustaceans, with up to a half-dozen subtypes on a single species [78]. The lateral antennular flagellum of the freshwater crayfish, Procambarus clarkii, contains a number of subtypes of setae which are all thought to function as hydrodynamic or bimodal hydrodynamic-chemosensory sensilla, but the exact functional properties of these structures are not fully known [7]. The crayfish, P. clarkii, is an ideal model organism to examine antennular sensilla functioning. Its 1st antennae (antennules) are highly sensitive to water-borne odors and mechanical disturbances and its brain is largely devoted to processing olfactory information. Likewise, these local interneurons in the deutocerebrum (midbrain) are large and can be readily accessed, from which electrical responses can be routinely recorded [68, 49].

\subsubsection{Antennule flicking and mechanoreception in crustaceans}

Almost all decapod and stomatopod crustaceans flick their antennules, which facilitates the transport of odorant molecules to the surface of the olfactory sensilla present on the antennules $[47,96,29]$. Such a motion constitutes a rapid forward-stroke which enhances the movement of odorants through the sensillar hairs, followed by a slower return-stroke which

results in odorant laden fluid being trapped between the sensilla [13, 11, 29]. The entrapped molecules are then transported to the surface of the aesthetascs by small-scale advection and molecular diffusion [11]. Price and Ache [97] and Schmitt and Ache [47] found that antennular flicking in the spiny lobster Panulirus argus enhances the response of olfactory sensory neurons to water-borne odorants. This flicking not only enhances delivery of odorants 
to chemosensory sensilla, but sensing of hydrodynamic and mechanical disturbances from mechano-sensory sensilla is also important for detection of predators, mates, and varying environmental substrates [7].

Previous work has shown that hydrodynamic stimulation of the antennule can evoke a flicking reflex in the crayfish P. clarkii [30]. Flicking in P. clarkii is a phasic behavior and is generated by action potential bursts in a single motor neuron that controls the lateral flagellar depressor muscle. The return stroke is slower and produced through elastic energy stored from the downstroke, and released from an elastic ligament at the base of the antennule. Reflex flicking was found to be triggered by standing feathered sensilla which are near-field receptors linearly arrayed along the antennular flagella [31].

A rich diversity of receptors is found on the antennules of different crustaceans. Even within a species, up to half a dozen or more subtypes of setae can be found [78]. In P. clarkii, the kinds of setae found on the lateral antennular flagellum include standing feathered setae, simple asymmetric setae, small and large beaked setae and filamentous setae (Figure 4.1A. Antennular flagellum of Procambarus clarkii. B. SEM image of the filamentous sensilli C. SEM image of the beaked sensillum in background, filamentous sensillum in middle, and asymmetric sensillum in foreground. D. Feathered sensillum, (image modified from Mellon and Hamid [32]). Scale bar is $20 \mu \mathrm{m}$.figure.caption.46). Their population density ranges from dense to rare and function varies from highly sensitive near-field reception to bimodal contact chemo-mechanoreception [31, 98, 99]. Hydrodynamic stimulation of feathered sensilla shows that these sensilla are the most sensitive to impulsive stimuli originating close to the axial plane of the flagellum [32]. An examination of the SEM images of the sockets of a feathered sensilla reveals that there are preferred directional sensitivities, which are most sensitive at right angles to the hinge-like elongation of the sensillar base. Electrophysiological and behavioral studies show that both lateral and medial flagella respond to hydrodynamic cues [33]. Although Mellon and Hamid [32] confirmed that weak hydrodynamic stimuli are able to excite the feathered sensilla, the sensitivity of the various sensilla morphologies to 
hydrodynamic disturbance and their functional properties are largely unknown.

\subsubsection{Simultaneous odorant and flow sensing}

From behavioral studies with aquatic animals, there is increasing evidence that both chemosensory and hydrodynamic sensory inputs are required to determine odor source location $[34,44,76]$. Chemical odorants are dispersed throughout aquatic environments through convection and diffusion with the flow and the distribution of odorants across the environment is inherently complex. Being scalar quantities, tracking odorants to their source requires input from other sensory systems as well, such as hydrodynamic sensors in addition to olfactory sensory organs. For Procambarus clarkii, local deutocerebral interneurons integrate hydrodynamic and odorant inputs, and the response is enhanced when both flow and odorant stimulation occurs together [68]. Both flow and odor signals have been suggested to aid in search behavior [34]. Numerous behavioral studies have now shown that hydrodynamic features are important, if not essential, for odor source detection by aquatic vertebrates and invertebrates alike $[100,3,34,101,6,42,102,76,103,95]$. Hydrodynamic stimulation of the antennules, including flicking [75, 7] and from ambient current evokes electrical activity in the central brain neurons $[68,48,49]$. Peak responses of olfactory receptor neurons occur when the aesthetasc responds to a combination of odorant and hydrodynamic stimulus $[68,76,77]$. Concentration and flow cues simultaneously excite chemoreceptors and mechanoreceptors of the antennules during flicking. In addition, many invertebrate organisms, including the spiny lobster P. argus [78], and crayfish P. clarkii [7], contain bimodal chemo-mechanoreceptors on their antennules that respond to both odorants and flow. For P. clarkii, local deutocerebral interneurons integrate hydrodynamic and odorant inputs, and the response of these central elements is enhanced when both flow and odorant stimulation occurs together [68]. 


\subsubsection{Research objectives}

This study aims to build on the findings of Mellon and Hamid [32] on hydrodynamic sensitivity of near-field sensilla in P. clarkii. The goal of this study is to determine the effects of hydrodynamic perturbation on flow and pressure distribution near an organism and the structural response of various hydrodynamic sensilla to the perturbation. We also attempt to examine the variation of the response to directionality of the hydrodynamic perturbation and its correlation to nerve spiking thresholds as reported by Mellon and Hamid [32]. The specific questions that are addressed in this study are:

1. What is the effect of upstream perturbation on pressure and velocity around the sensilla of downstream animal?

2. What effect does morphology have on force, moment and deformation of sensilla in aquatic organisms?

3. How does sensilla deformation vary with direction of incoming perturbation?

We utilize atomic force microscopy $(\mathrm{AFM})$ to characterize the torsional stiffness of sensilla and then utilize this information to calculate the response of flow and pressure profile on the sensilla surface to a pulse of motion upstream using a computational fluid dynamics (CFD) model. The resultant flow profile is applied to the sensilla hairs of four dominant morphologies to examine their structural bending response to the force exerted by the incoming flow. The results of the response are shown to have close correlation to nerve spiking response in the olfactory lobe of the animal brain. 


\subsection{Methods}

\subsubsection{Mechanosensory sensilla morphologies}

Scanning electron micrographs (SEM) were obtained from excised lateral antennular flagella of small $(5 \mathrm{~cm})$ postmolt or intermolt animals (as described in Mellon and Hamid, 2012 [32]). Flagella were removed surgically from the crayfish, and fixed overnight at $4{ }^{\circ} \mathrm{C}$ in $2 \%$ paraformaldehyde- $2 \%$ glutaraldehyde in $0.1 \mathrm{~mol} \mathrm{l}^{-1}$ sodium phosphate buffer ( $\mathrm{pH} 7.4$ ). Fixed flagella were washed 3 times in $0.1 \mathrm{~mol} \mathrm{l}^{-1}$ phosphate buffer, dehydrated in an ethanol series and critical-point dried prior to being gold coated and imaged on a JEOL 6400 scanning electron microscope (JEOL Ltd, Tokyo, Japan). Animals used for imaging were large adult southern swamp crayfish, Procambarus clarkii (Girard 1852), obtained from a commercial source in Louisiana (Atchafalaya Biological Supply, Raceland, LA, USA).

The four most dominant morphologies of mechanosensory sensilla (Figure 4.1A. Antennular flagellum of Procambarus clarkii. B. SEM image of the filamentous sensilli C. SEM image of the beaked sensillum in background, filamentous sensillum in middle, and asymmetric sensillum in foreground. D. Feathered sensillum, (image modified from Mellon and Hamid [32]). Scale bar is $20 \mu$ m.figure.caption.46) found on P. clarkii are the feathered, beaked, filamentous, and asymmetric types [7]. These four morphologies were imaged using Scanning Electron Micrographs (SEM) and their characteristic dimensions, including length, diameter, and hair structure are listed in Table 4.1Dimensions and properties of various morphologies of sensilla found on a crayfish antennuletable.caption.47. These morphologic characteristics were used within a computational fluid dynamics (CFD) and fluid-structure interaction (FSI) numerical model to determine the forces, moments, and bending along each of these morphologically distinct sensilla. The simulated shapes of the sensilla used in these models, created by computer-aided design (CAD) from dimensions obtained from the SEM images, are shown in Figure 4.2Idealized CAD models of four primary morphologies of sensilla found on crayfish antennules: (A) Feathered, (B) Beaked, (C) Filamentous and (D) Asymmetric 


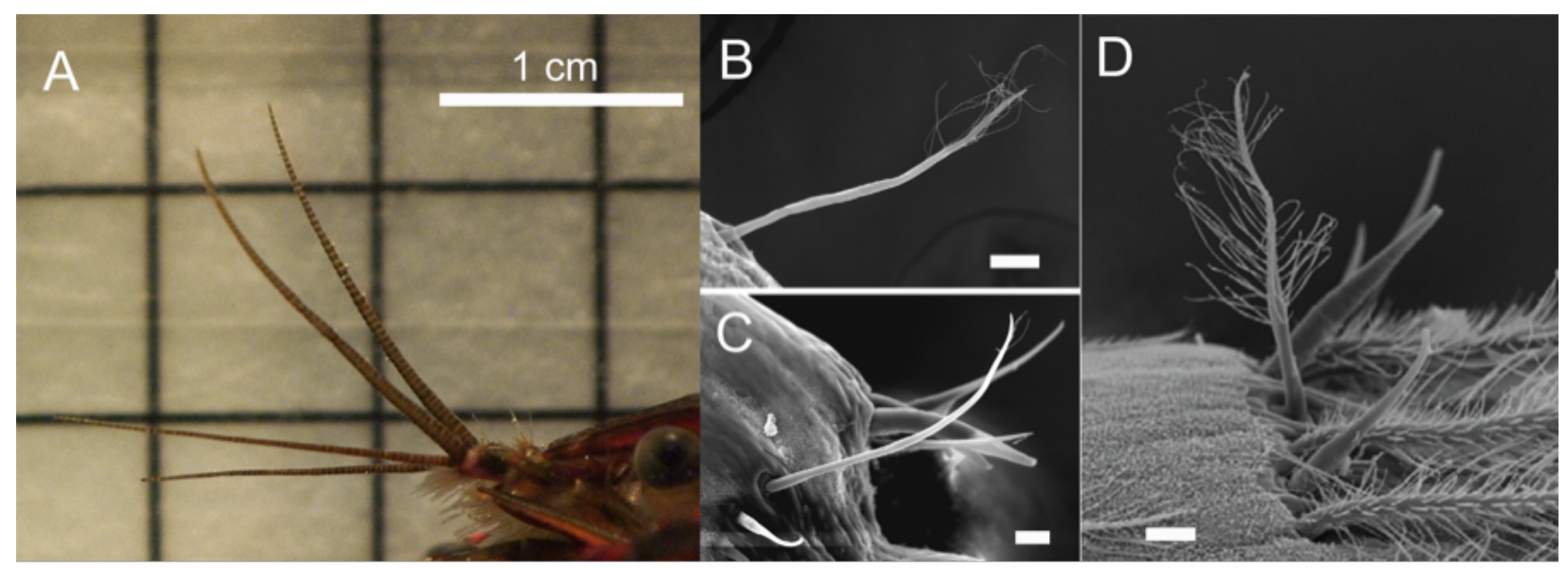

Figure 4.1: A. Antennular flagellum of Procambarus clarkii. B. SEM image of the filamentous sensilli C. SEM image of the beaked sensillum in background, filamentous sensillum in middle, and asymmetric sensillum in foreground. D. Feathered sensillum, (image modified from Mellon and Hamid [32]). Scale bar is $20 \mu \mathrm{m}$.

sensilla. The scale bars are $25 \mu$ m.figure.caption.48.

Table 4.1: Dimensions and properties of various morphologies of sensilla found on a crayfish antennule

\begin{tabular}{lllll}
\hline Morphology & Length $(\mu \mathrm{m})$ & Diameter $(\mu \mathrm{m})$ & $k_{t, a v e}(\mathrm{~N}-\mathrm{m} / \mathrm{rad})$ & Sensing mode \\
\hline Feathered & 150 & 5 tapering to 1 & & Hydrodynamic \\
Beaked & 150 & 15 tapering to 5 & $3 \times 10^{-12}$ & Hydrodynamic and chemical \\
Asymmetric & 80 & 10 tapering to 1 & Hydrodynamic and chemical \\
Filamentous & 200 & 10 tapering to 1 & Hydrodynamic \\
\hline
\end{tabular}

\subsubsection{Upstream flow perturbation response}

To determine the perturbation in flow and pressure caused by an upstream disturbance, a two-dimensional CFD model of a pulsating flow is employed and the response is recorded adjacent to the antennule surface. The ANSYS ${ }^{\mathrm{TM}}$ multi-physics software package is used to first solve for the flow and pressure distribution around the antennule (Figure 4.3CFD model for velocity and pressure response at the surface of antennule B $(d=0.5 \mathrm{~mm})$ caused by a pulse movement of the upstream circle $\mathrm{A}(D=9.5 \mathrm{~mm})$. The graph shows the pulse displacement of circle A and the velocity and pressure profile at the sensilla attached to the antennule (circle B) at angle $\theta$ are used as inlet conditions for the sensilla CFD models.figure.caption.49), which 

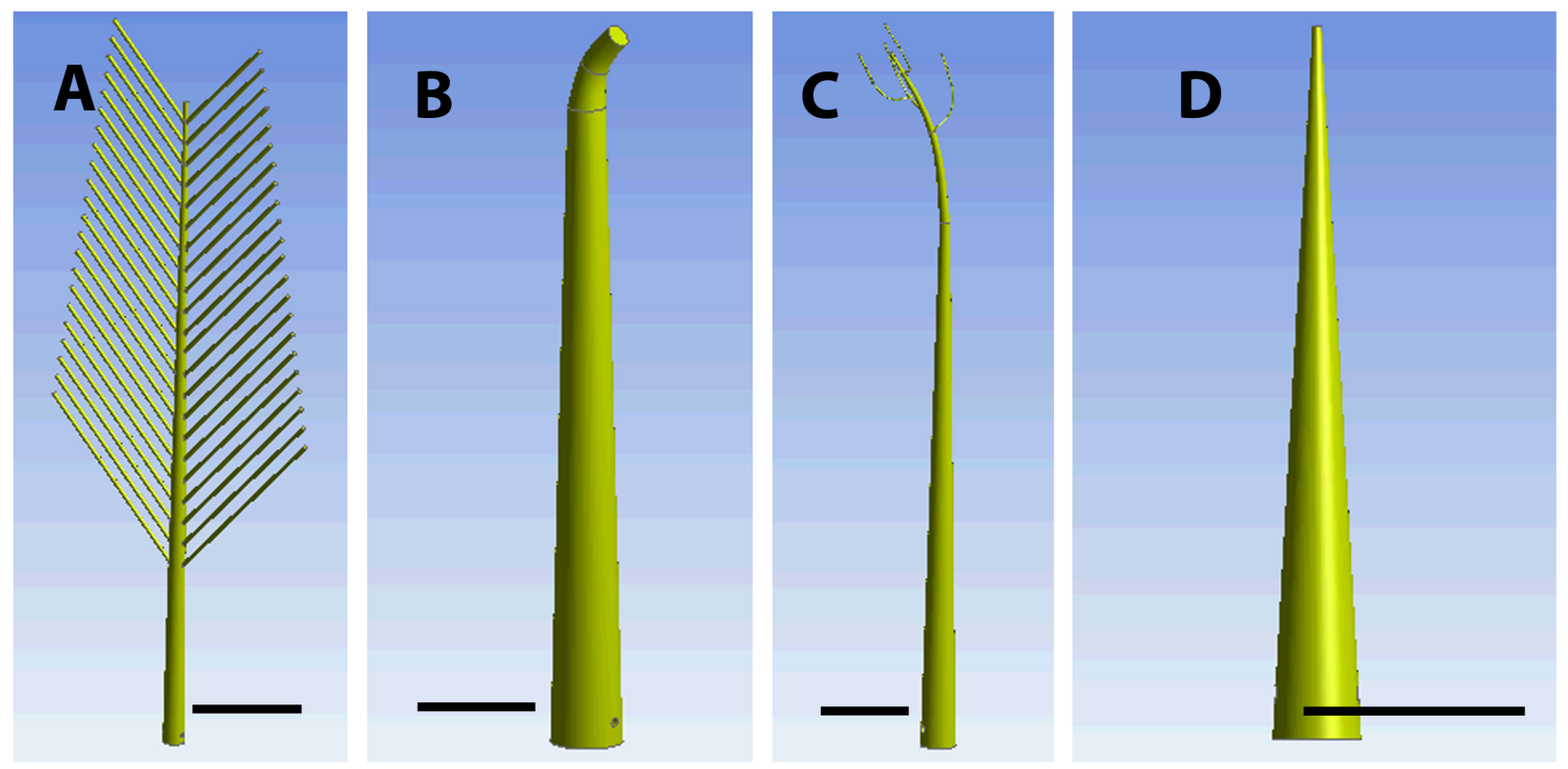

Figure 4.2: Idealized CAD models of four primary morphologies of sensilla found on crayfish antennules: (A) Feathered, (B) Beaked, (C) Filamentous and (D) Asymmetric sensilla. The scale bars are $25 \mu \mathrm{m}$.

is then inputted into the fluid-structure interaction model (Figure 4.4(A) CFD model of the feathered sensilla. Inlet flow into the model is derived from the two-dimensional perturbation model. (B) A revolute joint at the proximal end of the sensilla allows it to rotate around the joint by an angle $\delta$ under the pressure of incoming flow. (C) Flowchart for computing the upstream fluid perturbation using the CFD model, which is then used to determine flow and pressure distribution around the sensilla. This pressure load is then inputted into the FSI model to compute angular deflection along the base of the sensilla.figure.caption.50A and 4.4(A) CFD model of the feathered sensilla. Inlet flow into the model is derived from the two-dimensional perturbation model. (B) A revolute joint at the proximal end of the sensilla allows it to rotate around the joint by an angle $\delta$ under the pressure of incoming flow. (C) Flowchart for computing the upstream fluid perturbation using the CFD model, which is then used to determine flow and pressure distribution around the sensilla. This pressure load is then inputted into the FSI model to compute angular deflection along the base of the sensilla.figure.caption.50B). The upstream perturbation was created by a circular object of $9.5 \mathrm{~mm}$ diameter and was modeled to mimic the perturbation formed to quantify 


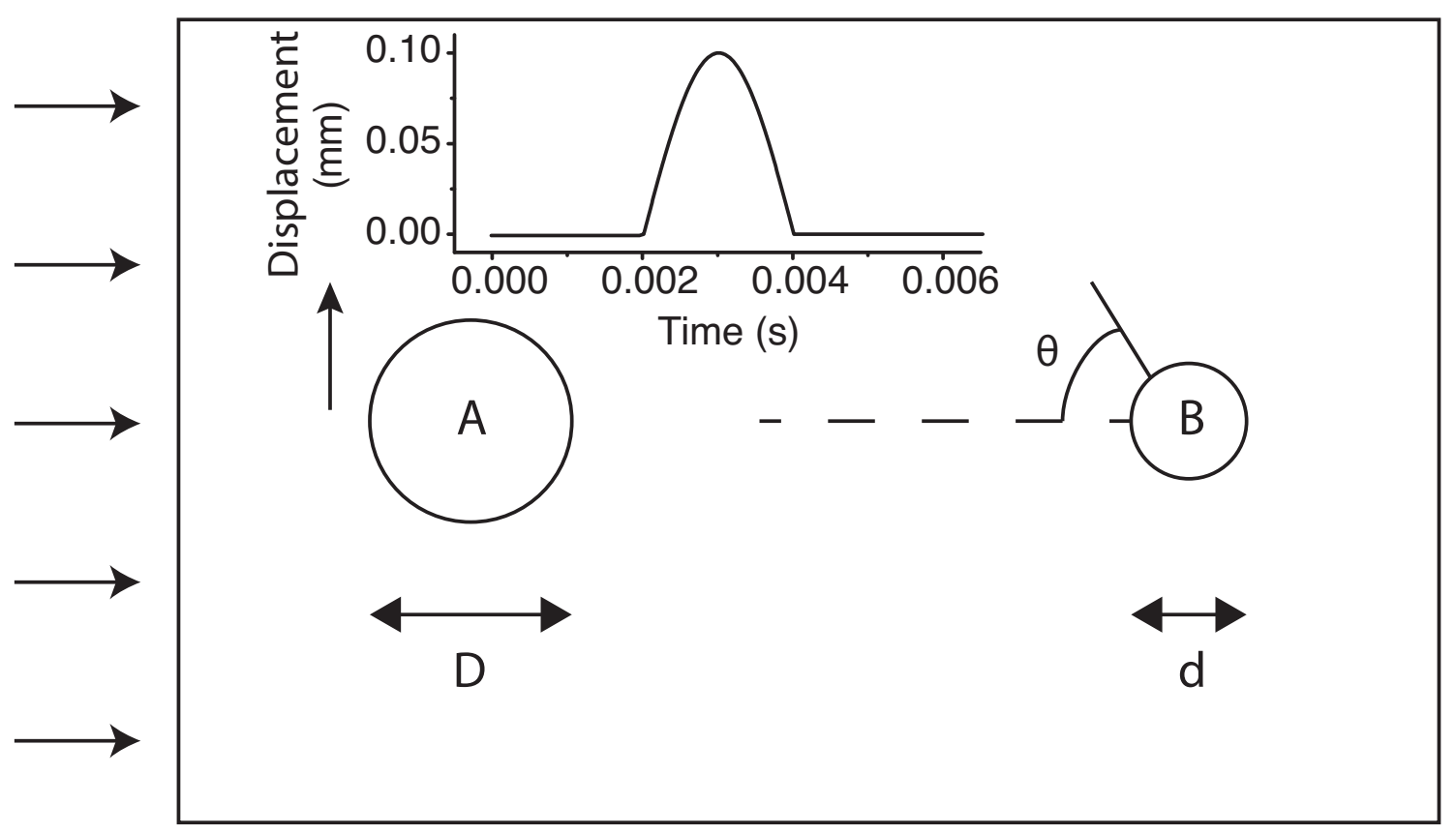

Figure 4.3: CFD model for velocity and pressure response at the surface of antennule B $(d=0.5 \mathrm{~mm})$ caused by a pulse movement of the upstream circle A $(D=9.5 \mathrm{~mm})$. The graph shows the pulse displacement of circle A and the velocity and pressure profile at the sensilla attached to the antennule (circle B) at angle $\theta$ are used as inlet conditions for the sensilla CFD models.

neural spiking thresholds of standing feathered sensilla by Mellon and Hamid [32]. In the neural spiking threshold experiments, a $9.5 \mathrm{~mm}$ diameter sphere was used to create a flow disturbance, which generated a flow-disturbance pulse which lasted $2 \mathrm{~ms}$. In the model, the circular object was displaced the same distances and over the same time periods as the sphere within the neural spiking threshold experiments (Figure 4.3CFD model for velocity and pressure response at the surface of antennule B $(d=0.5 \mathrm{~mm})$ caused by a pulse movement of the upstream circle A $(D=9.5 \mathrm{~mm})$. The graph shows the pulse displacement of circle A and the velocity and pressure profile at the sensilla attached to the antennule (circle B) at angle $\theta$ are used as inlet conditions for the sensilla CFD models.figure.caption.49). 


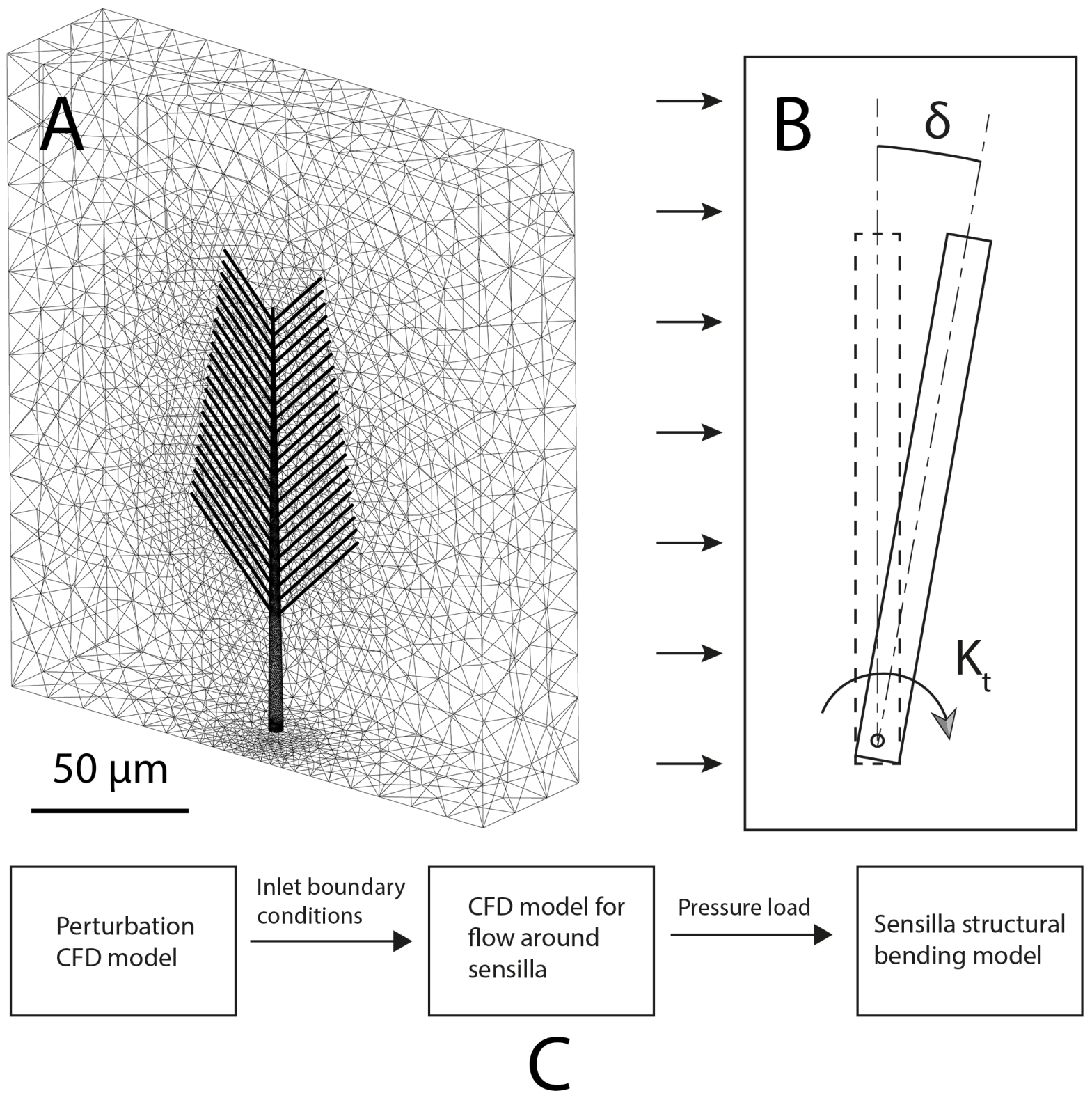

Figure 4.4: (A) CFD model of the feathered sensilla. Inlet flow into the model is derived from the two-dimensional perturbation model. (B) A revolute joint at the proximal end of the sensilla allows it to rotate around the joint by an angle $\delta$ under the pressure of incoming flow. (C) Flowchart for computing the upstream fluid perturbation using the CFD model, which is then used to determine flow and pressure distribution around the sensilla. This pressure load is then inputted into the FSI model to compute angular deflection along the base of the sensilla.

The model solves for a 2D transient incompressible flow around the circular entities for which the governing equations are: 


$$
\begin{aligned}
& \frac{\partial u}{\partial t}+u \frac{\partial u}{\partial x}+v \frac{\partial u}{\partial y}=-\frac{1}{\rho} \frac{\partial p}{\partial x}+\nu\left(\frac{\partial^{2} u}{\partial x^{2}}+\frac{\partial^{2} u}{\partial y^{2}}\right) \\
& \frac{\partial v}{\partial t}+u \frac{\partial v}{\partial x}+v \frac{\partial v}{\partial y}=-\frac{1}{\rho} \frac{\partial p}{\partial y}+\nu\left(\frac{\partial^{2} v}{\partial x^{2}}+\frac{\partial^{2} v}{\partial y^{2}}\right)
\end{aligned}
$$

where $u, v$ and $p$ are $x$-velocity, $y$-velocity and pressure respectively. The following boundary conditions are applied for the model:

- Inlet: $u=U_{0}(1.8 \mathrm{~cm} / \mathrm{s}$, which is the typical flicking speed for crayfish), $v=0$

- Top and bottom: zero shear slip walls are applied, i.e. $v=0, \partial p / \partial y=0, \partial u / \partial y=0$.

- Outlet: A constant pressure outlet is used: $p=p_{a t m}$

- Circular walls: A no-slip wall boundary condition is applied: $u=v=0$.

A rectangular flow domain of size $200 d$ ( $d=$ diameter of the downstream circle) was used. A mesh size of 3500 cells was required to converge the results and capture the flow dynamic for the model. The commercial solver $\mathrm{CFX}^{\mathrm{TM}}$ was used to solve for velocity and pressure throughout the flow domain. The results from this study are then used as inlet boundary conditions for deformation analysis of the sensilla located on the downstream antennule within the fluid-structure interaction model (Figure 4.4(A) CFD model of the feathered sensilla. Inlet flow into the model is derived from the two-dimensional perturbation model. (B) A revolute joint at the proximal end of the sensilla allows it to rotate around the joint by an angle $\delta$ under the pressure of incoming flow. (C) Flowchart for computing the upstream fluid perturbation using the CFD model, which is then used to determine flow and pressure distribution around the sensilla. This pressure load is then inputted into the FSI model to compute angular deflection along the base of the sensilla.figure.caption.50A and 4.4(A) CFD model of the feathered sensilla. Inlet flow into the model is derived from the two-dimensional perturbation model. (B) A revolute joint at the proximal end of the sensilla allows it to rotate around 
the joint by an angle $\delta$ under the pressure of incoming flow. (C) Flowchart for computing the upstream fluid perturbation using the CFD model, which is then used to determine flow and pressure distribution around the sensilla. This pressure load is then inputted into the FSI model to compute angular deflection along the base of the sensilla.figure.caption.50B). Figure 4.4(A) CFD model of the feathered sensilla. Inlet flow into the model is derived from the two-dimensional perturbation model. (B) A revolute joint at the proximal end of the sensilla allows it to rotate around the joint by an angle $\delta$ under the pressure of incoming flow. (C) Flowchart for computing the upstream fluid perturbation using the CFD model, which is then used to determine flow and pressure distribution around the sensilla. This pressure load is then inputted into the FSI model to compute angular deflection along the base of the sensilla.figure.caption.50C displays the connectivity of models where the results from the perturbation model (Figure 4.3CFD model for velocity and pressure response at the surface of antennule B $(d=0.5 \mathrm{~mm})$ caused by a pulse movement of the upstream circle $\mathrm{A}(D=9.5 \mathrm{~mm})$. The graph shows the pulse displacement of circle A and the velocity and pressure profile at the sensilla attached to the antennule (circle B) at angle $\theta$ are used as inlet conditions for the sensilla CFD models.figure.caption.49) are used as boundary conditions for the CFD portion of FSI (Figure 4.4(A) CFD model of the feathered sensilla. Inlet flow into the model is derived from the two-dimensional perturbation model. (B) A revolute joint at the proximal end of the sensilla allows it to rotate around the joint by an angle $\delta$ under the pressure of incoming flow. (C) Flowchart for computing the upstream fluid perturbation using the CFD model, which is then used to determine flow and pressure distribution around the sensilla. This pressure load is then inputted into the FSI model to compute angular deflection along the base of the sensilla.figure.caption.50A). The flow field generated by the flow around the sensilla is then applied as a pressure load on the sensilla to compute its deformation around its base, $\delta$ (Figure 4.4(A) CFD model of the feathered sensilla. Inlet flow into the model is derived from the two-dimensional perturbation model. (B) A revolute joint at the proximal end of the sensilla allows it to rotate around the joint by an angle $\delta$ under the pressure of incoming 
flow. (C) Flowchart for computing the upstream fluid perturbation using the CFD model, which is then used to determine flow and pressure distribution around the sensilla. This pressure load is then inputted into the FSI model to compute angular deflection along the base of the sensilla.figure.caption.50B). This method constitutes a one-way FSI formulation where the flow field around the sensilla affects its structural position but not vice-versa. This formulation is justified since the deflection of the sensilla, $\delta \ll 1$ degree and hence does not effect the flow field significantly. The CFD domain in Figure 4.4(A) CFD model of the feathered sensilla. Inlet flow into the model is derived from the two-dimensional perturbation model. (B) A revolute joint at the proximal end of the sensilla allows it to rotate around the joint by an angle $\delta$ under the pressure of incoming flow. (C) Flowchart for computing the upstream fluid perturbation using the CFD model, which is then used to determine flow and pressure distribution around the sensilla. This pressure load is then inputted into the FSI model to compute angular deflection along the base of the sensilla.figure.caption.50A was generated for each of the four sensilla morphologies. To achieve mesh density convergence, the feathered sensilla flow domain was meshed into $\sim 2$ million nodes with tetrahedral dominant elements. The asymmetric, beaked and filamentous models required $\sim 38000,24000$ and 370000 nodes respectively.

\subsubsection{Sensilla bending analysis}

Within the fluid-structure interaction model, sensilla are assumed to be attached to the antennule at its proximal end by a revolute joint with a torsional stiffness, $k_{t}$. The sensilla itself is modeled as a structurally rigid body hinged at its base, since the AFM measurements show little bending along the length of the sensilla as described in the section "computation of torsional stiffness". A no-slip wall is applied at the bottom of the flow domain, which is the surface of the antennule. A constant pressure is applied at the outlet and zero shear slip walls are assumed to be present at the remaining three sides. The force and bending moment on the sensilla resulting from the incoming flow are calculated by integrating the resultant 
pressure over the surface of the sensilla. The effect of relative angular position of the upstream flow perturbation on the sensilla response is studied by adjusting the angular position of the sensilla on the antennule, $\theta$ (Figure 4.3CFD model for velocity and pressure response at the surface of antennule B $(d=0.5 \mathrm{~mm})$ caused by a pulse movement of the upstream circle A $(D=9.5 \mathrm{~mm})$. The graph shows the pulse displacement of circle A and the velocity and pressure profile at the sensilla attached to the antennule (circle B) at angle $\theta$ are used as inlet conditions for the sensilla CFD models.figure.caption.49) in steps of $20^{\circ}$, and then solving the model for pressure and angle of deflection for each sensilla type.

\subsubsection{Computation of torsional stiffness}

The torsional stiffness $k_{t}$ for the sensilla was calculated using data from atomic force microscopy experiments. Samples were prepared in a manner similar to that described above and prepared for in-fluid AFM experiments. The AFM was first used in contact mode with a fluid cell to probe the sensilla surface and generate a topography. System thermal equilibrium was achieved before beginning and a $5 \times 5 \mu \mathrm{m}$ region was scanned with scan parameters (rate, set-point force, etc.) optimized for scan quality. NP-20 probes were used, with nominal cantilever stiffness of $0.06 \mathrm{~N} \mathrm{~m}^{-1}$ and a tip radius of 20-50 nm. Several sets of experiments were performed at different locations on the sensilla, one near the base and one further towards the distal end, and one more roughly in the middle of the sensilla. The force, $F$, needed to deflect the sensilla a distance, $d_{s}$, was quantified for all locations and the angle of rotation was quantified as $\theta_{s}=L d_{s}^{-1}$, where $L$ is the moment arm or distance from the proximal end of the sensilla and small angular deflection is assumed. The torsional stiffness was computed using the basic mechanics relation for moment balance at the hinge, and assuming the sensilla responded as a rigid body throughout the AFM experiments:

$$
k_{t} \theta_{s}=F L
$$


The force-deflection data collected at points along the sensilla were essentially linear, suggesting that the assumption of a rigid body rotation hinged at the base is valid. For a small angle of deformation, the torsional stiffness at the base of the sensilla can be quantified as:

$$
k_{t}=F L^{2} d_{s}^{-1}
$$

A mean estimate of $k_{t}=1 \times 10^{-12} \mathrm{~N}-\mathrm{m}$ degree $^{-1}$ was determined from a total of 87 measurements on 11 different sensilla.

\subsection{Results}

\subsubsection{Upstream perturbation response}

The effects of an upstream hydrodynamic perturbation on water velocity and pressure along a P. clarkii antennule are studied using a two-dimensional CFD model. The circle upstream undergoes a pulse movement for a duration of 2ms, as shown in Figure 4.3CFD model for velocity and pressure response at the surface of antennule B $(d=0.5 \mathrm{~mm})$ caused by a pulse movement of the upstream circle $\mathrm{A}(D=9.5 \mathrm{~mm})$. The graph shows the pulse displacement of circle A and the velocity and pressure profile at the sensilla attached to the antennule (circle B) at angle $\theta$ are used as inlet conditions for the sensilla CFD models.figure.caption.49. This movement matches the same experimental setup used to stimulate near-field mechanoreceptors on P. clarkii and determine thresholds of sensory neuron responses in Mellon and Hamid [32]. Water velocity and pressure are quantified adjacent to the surface of the antennule where a sensilla would normally be present. The Reynolds number for the antennule is $\sim 9$ and $R e$ for the sensilla is $\sim 3$. The hydrodynamic pulse results in a sharp peak in velocity of $0.85 \mathrm{~cm} \mathrm{~s}^{-1}$ followed by a sharp drop to $0.78 \mathrm{~cm} \mathrm{~s}^{-1}$ before returning to a the mean flow speed 
of $0.81 \mathrm{~cm} \mathrm{~s}^{-1}$ (Figure $4.5(\mathrm{~A})$ Velocity and pressure response along the sensilla located on the downstream circle as the upstream sphere creates a pulse of $2 \mathrm{~ms}$ duration. Pressure contours (B) before and (C) after the pulse motion of the upstream circular entity. figure.caption.51). The peak and the trough in velocity closely correspond to peaks in pressure of 3 and $4 \mathrm{~Pa}$ respectively above the background pressure. The results from this model show that a sudden pulse of movement upstream results in a momentary increase in velocity and pressure along the antennule and at locations of mechanosensory sensilla.

\subsubsection{Sensilla response to perturbed flow}

The flow fields around the various sensilla morphologies are shown in Figure 4.6Flow velocity contours around the sensilla at two different heights from the base: A. asymmetric sensilla at $z=20$ and $60 \mu \mathrm{m}, \mathrm{B}$. beaked sensilla at $z=60$ and $140 \mu \mathrm{m}, \mathrm{C}$. feathered sensilla at $z=60$ and $120 \mu \mathrm{m}$ and D. filamentous sensilla at $z=70$ and $190 \mu \mathrm{m}$. The contours are colored by velocity and the sensilla surface is colored by magnitude of deformation of the sensilla from its original position.figure.caption.52. The sensilla walls are colored by total displacement of the sensilla surface and the streamlines indicate water velocities at fixed distances from the base for all 4 morphologies. As the sensilla are attached to the antennule surface through joints, simulated as revolute joints in the FSI model, the sensilla responds to the incoming flow by rotating around the joint. A greater surface area exposed to the flow or a longer length of the sensilla results in larger bending moment applied to it around the joint and resultantly larger

angular deformation, $\delta$ (Figure 4.7Angular deformation of the sensilla $\delta$, force exerted by the incoming flow and the corresponding bending moment for different sensilla morphologies: asymmetric, beaked, feathered and filamentous.figure.caption.53). The filamentous sensilla morphology, which is the longest $(200 \mu \mathrm{m})$ experiences the maximum deformation of $0.91^{\circ}$. This is approximately 66 times greater than the deformation of the asymmetric sensilla morphology, which has the least deformation. The feathered sensilla, which has the greatest surface area, experiences the maximum total force and bending moment, which is 10 times 
the force and 23 times the bending moment applied to the asymmetric sensilla. However, since the feathered sensilla is shorter than the filamentous sensilla, its deformation is less. In terms of water velocities surrounding the sensilla, the branching structure of the feathered, and to a lesser extent the filamentous, contributes to the overall drag force that the sensilla experiences. Due to the low Reynolds number regime of the flow, a greater amount of fluid flows around the feathered sensilla than through its branches. Such 'paddle-type' behavior [11] provides much greater resistance to the flow, leading to a larger circulation region in the wake of the sensilla.

\subsubsection{Direction of perturbation}

The effects of the direction of incoming flow perturbation on the sensilla response was determined by varying the angle $\theta$ (Figure 4.4(A) CFD model of the feathered sensilla. Inlet flow into the model is derived from the two-dimensional perturbation model. (B) A revolute joint at the proximal end of the sensilla allows it to rotate around the joint by an angle $\delta$ under the pressure of incoming flow. (C) Flowchart for computing the upstream fluid perturbation using the CFD model, which is then used to determine flow and pressure distribution around the sensilla. This pressure load is then inputted into the FSI model to compute angular deflection along the base of the sensilla.figure.caption.50) from $0^{\circ}$ to $360^{\circ}$ in increments of $20^{\circ}$. The incoming flow approaches the downstream circle at $\theta=0^{\circ}$ and $\theta=180^{\circ}$ corresponds to the center of its wake. The water velocity around the sensilla increases to a maximum of 1.15 $\mathrm{cm} \mathrm{s}^{-1}$ as the angle $\theta$ increases from 0 to $90^{\circ}$ and is the smallest $\left(0.43 \mathrm{~cm} \mathrm{~s}^{-1}\right)$ at $180^{\circ}$, when the flow perturbation approaches the sensilla from the opposite direction (Figure 4.8Peak velocity of the incoming flow around the sensilla for various positions, $\theta$ around the circumference of the antennule.figure.caption.54). The velocities and corresponding pressure distributions along the sensilla have direct correlation to the variation of nerve spike threshold with the direction of perturbation (Figure 4.9Polar plot of normalized sensillar spiking thresholds in P. clarkii as a function of the relative position of a $5 \mathrm{~ms}$ near-field stimulus. Data are means \pm 1 
s.e.m. of 25 sensilla. The 0 degree position was directly ahead of (distal to) the sensillum under observation. The stimulus probe was moved sequentially in 20 degree increments to 17 different positions clockwise and counterclockwise around the sensillum. (Adapted from Mellon and Hamid [32]).figure.caption.55, adapted from Mellon and Hamid [32]), suggesting that greater perturbations in fluid movement surrounding the antennule, and consequently sensilla deformation $\delta$, lead to increased neural response in the animals brain. Angular deformation for various sensilla morphologies is compared in Figure 4.10Angular deformation, $\delta$ of the sensilla at positions around the antennule for different morphologies: A. asymmetric, B. beaked, C. feathered and D. filamentousfigure.caption.56. Results show that deformation variation with direction of perturbation closely follows the flow speed the sensilla are exposed to, with greatest deformation observed in feathered sensilla oriented at right angles to the direction of perturbation.

\subsubsection{Model sensitivity to torsional stiffness}

An average value of $k_{t}=1 \times 10^{-12} \mathrm{~N} . \mathrm{m} /$ degree was used for the models as calculated from AFM measurements for the beaked sensilla. The sensitivity of the model to torsional stiffness for each of the morphologies is determined by varying $k_{t}$ over several orders of magnitude and calculating the deformation response for each case. Results (Figure 4.11Sensitivity of sensilla deformation to variation in torsional stiffness, $k_{t}$ over several orders of magnitude. The AFM measurements and value used in the model are for $k_{t}=1 \times 10^{-12} \mathrm{~N} . \mathrm{m} /$ degree. The plot shows little variation in deformation $(\delta)$ for less stiff sensilla $\left(k_{t}<1 \times 10^{-12} \mathrm{~N} . \mathrm{m} /\right.$ degree$)$ but a sharp reduction in angular deformation, and thus sensitivity of the sensilla to hydrodynamic disturbance, for sensilla that have greater torsional stiffness. figure.caption.57) show that the angular deformation of each of the morphologies is fairly insensitive to changes in torsional stiffness over about five orders of magnitude for smaller values of $k_{t}$. For stiffness values greater than $k_{t}=1 \times 10^{-12} \mathrm{~N} . \mathrm{m} /$ degree, there is a sharp decrease in angular deformation, suggesting that any stiffness greater than that measured from AFM, a reduction in the 

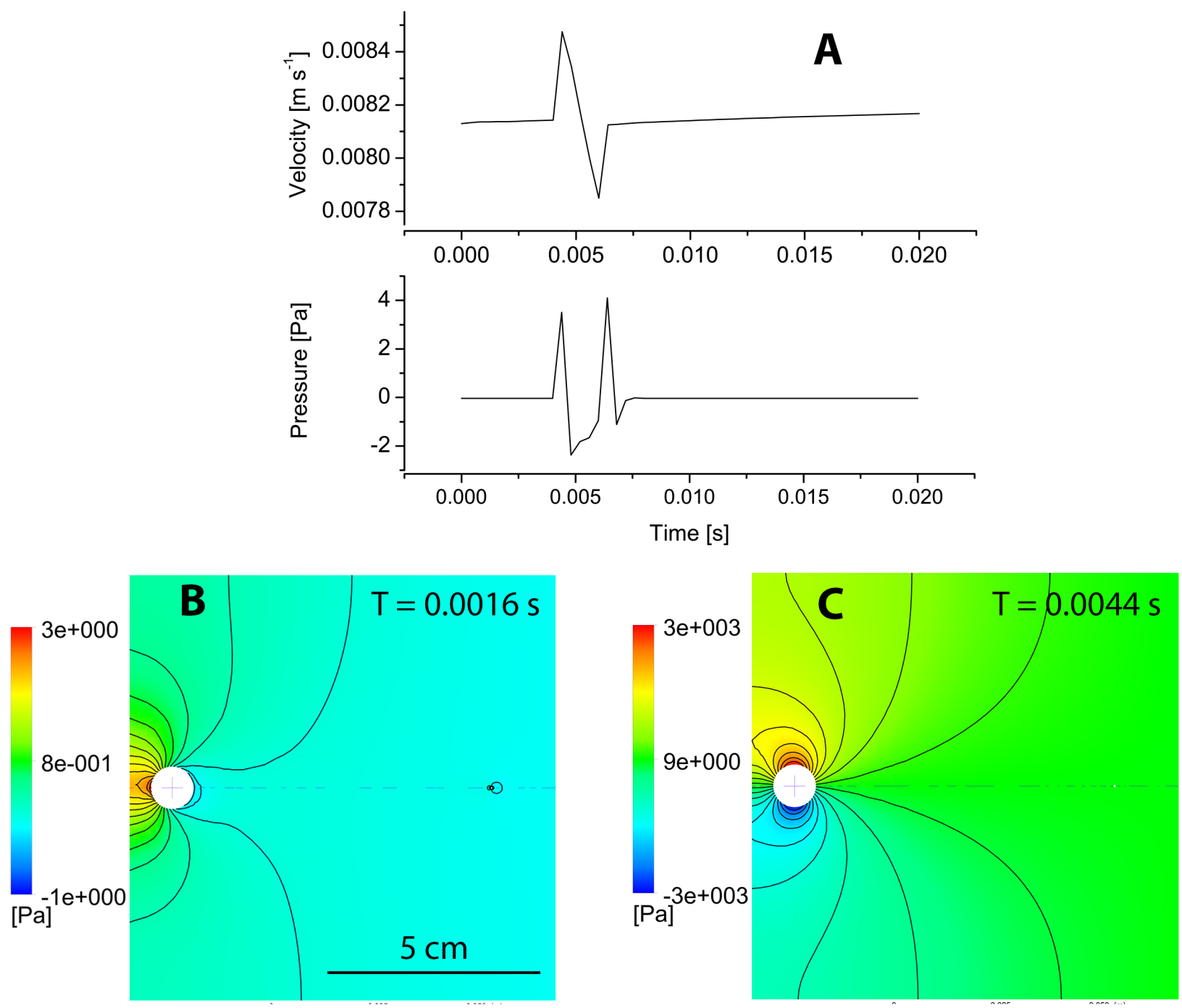

Figure 4.5: (A) Velocity and pressure response along the sensilla located on the downstream circle as the upstream sphere creates a pulse of $2 \mathrm{~ms}$ duration. Pressure contours (B) before and $(\mathrm{C})$ after the pulse motion of the upstream circular entity.

sensitivity of the sensilla occurs. In addition, there is a transition from the filamentous hairs being the most sensitive at $k_{t}=1 \times 10^{-12} \mathrm{~N}$.m/degree to the feathered sensilla being the most sensitive to angular deformation at $k_{t}=5 \times 10^{-11} \mathrm{~N} . \mathrm{m} /$ degree.

\subsection{Discussion}

The results presented in this study show that an upstream perturbation in flow can cause alterations in fluid velocities and the resultant pressure distribution surrounding sensilla 


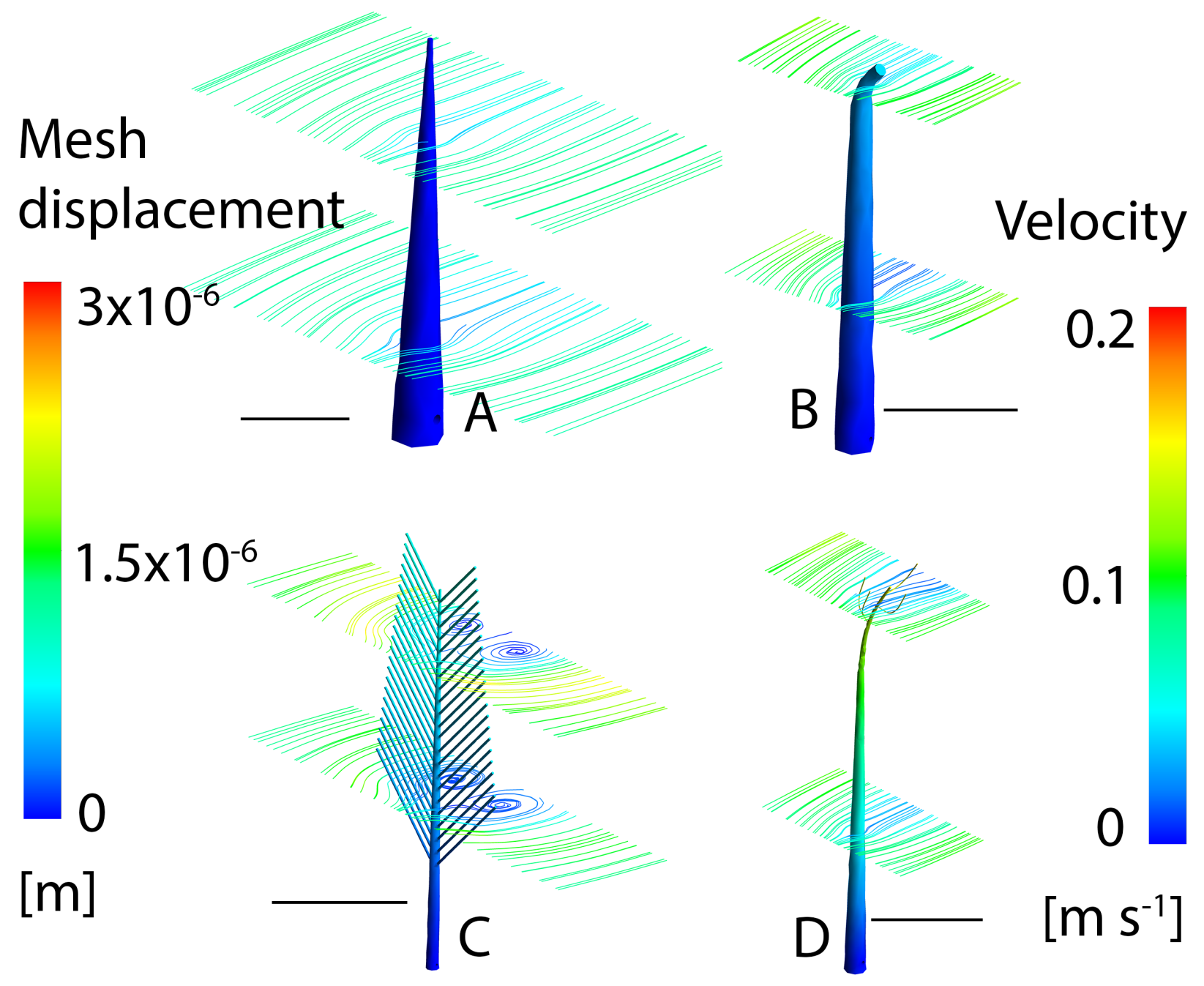

Figure 4.6: Flow velocity contours around the sensilla at two different heights from the base: A. asymmetric sensilla at $z=20$ and $60 \mu \mathrm{m}, \mathrm{B}$. beaked sensilla at $z=60$ and $140 \mu \mathrm{m}$, C. feathered sensilla at $z=60$ and $120 \mu \mathrm{m}$ and D. filamentous sensilla at $z=70$ and $190 \mu \mathrm{m}$. The contours are colored by velocity and the sensilla surface is colored by magnitude of deformation of the sensilla from its original position.

along the crayfish antennule. Of the morphologies tested, the standing feathered sensillum encounters the largest force and bending moment against the incoming flow while the feathered and filamentous morphology experiences similar angular deformations. Results also indicate that the sensilla undergo maximum angular deflection when aligned close to right angles to the direction of the perturbation. These hydrodynamic and pressure distribution results are in close conformity to sensitivity to direction of perturbed flow as measured by neuron spiking thresholds in the animal brain as reported in Mellon and Hamid [32]. 

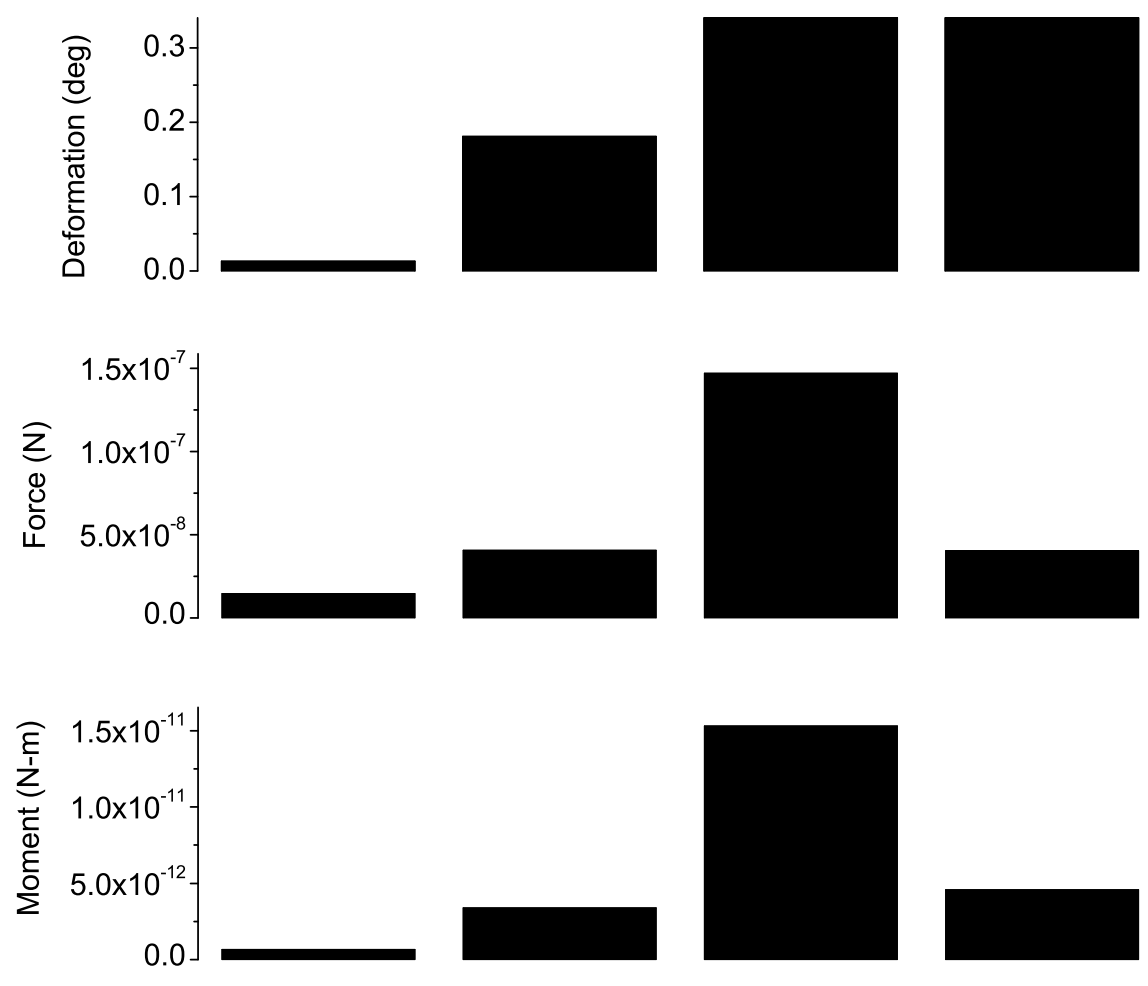

Beaked

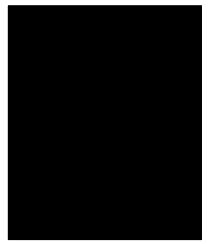

Feathered

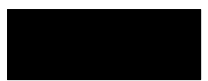

Filamentous

Figure 4.7: Angular deformation of the sensilla $\delta$, force exerted by the incoming flow and the corresponding bending moment for different sensilla morphologies: asymmetric, beaked, feathered and filamentous.

\subsubsection{Sensilla morphology and sensitivity}

As this upstream perturbation in the flow creates a hydrodynamic disturbance around the antennule, either due to antennule flicking, ambient currents, or turbulence induced by a nearby predator or mate, it generates a local pressure profile along the surface of the sensilla, and causes an angular deformation about its base. The incoming flow exerts a distributed velocity and pressure profile on the structure of the sensilla. The flow field around the sensilla is a result of different structural morphologies and determines the total pressure applied on the sensilla by the flow. Hydrodynamic sensilla have a variety of morphologies that allow varied degrees of sensitivity under different flow conditions. The standing feathered sensilla, due to its large surface area,dense array of branches, forces the majority of the flow to go 


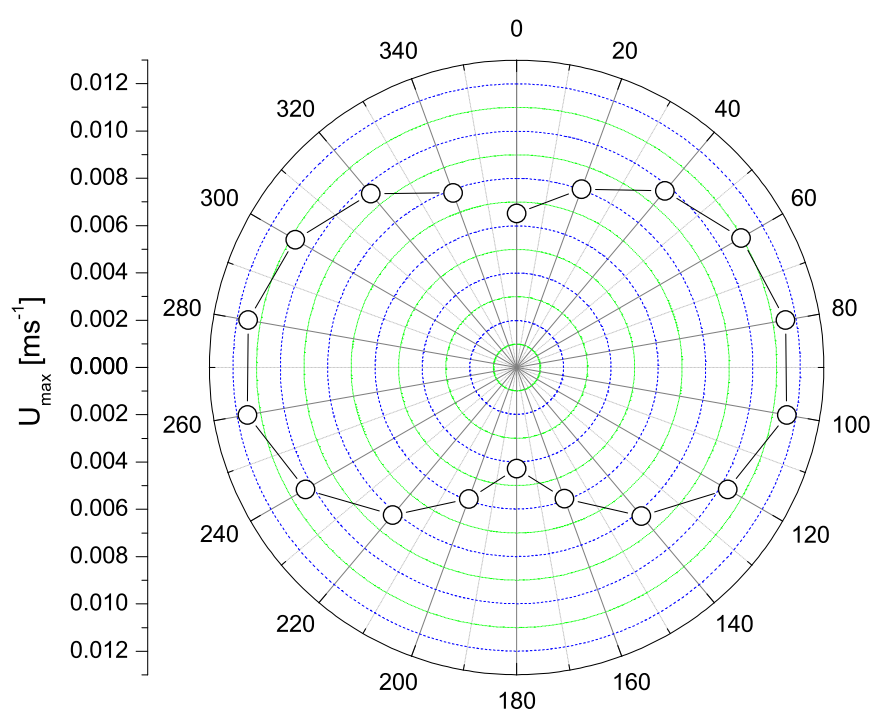

Figure 4.8: Peak velocity of the incoming flow around the sensilla for various positions, $\theta$ around the circumference of the antennule.

around its structure instead of through its branches (Figure 4.6Flow velocity contours around the sensilla at two different heights from the base: A. asymmetric sensilla at $z=20$ and 60 $\mu \mathrm{m}, \mathrm{B}$. beaked sensilla at $z=60$ and $140 \mu \mathrm{m}$, C. feathered sensilla at $z=60$ and $120 \mu \mathrm{m}$ and D. filamentous sensilla at $z=70$ and $190 \mu \mathrm{m}$. The contours are colored by velocity and the sensilla surface is colored by magnitude of deformation of the sensilla from its original position.figure.caption.52). Due to its morphology, in combination with the low Reynolds number laminar flow it experiences under small fluid perturbations, the feathered sensilla essentially acts as a paddle [11] and experiences the maximum force applied to its base joint among all predominant sensilla types. However, the bending moment about its base is similar in magnitude to the filamentous sensilla, which experiences less overall force, but due to its greater length, creates an equivalent angular deflection at its base. Both sensilla morphologies result in large deformations around the joint and allows detection of small perturbations in the flow field. The neural response of the feathered sensilla was studied by Mellon and Hamid [32] and the greatest extent of deformation around the joint was found to correlate with the largest direction of nerve spiking in the animal brain. This suggests that variations 


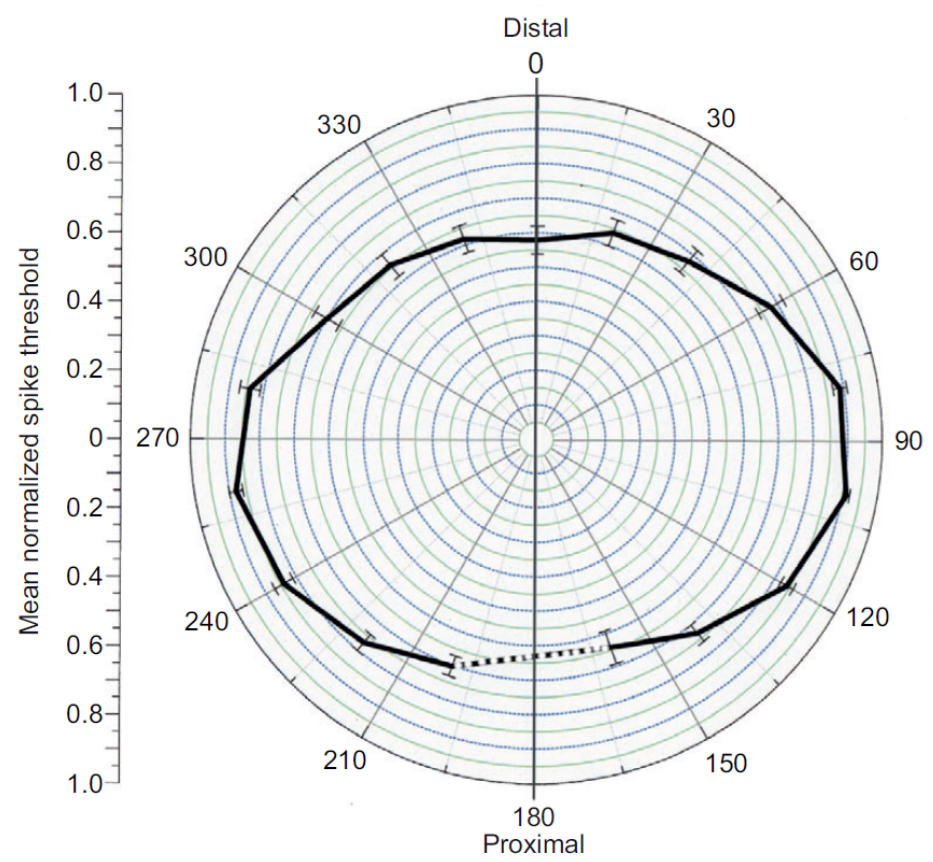

Figure 4.9: Polar plot of normalized sensillar spiking thresholds in P. clarkii as a function of the relative position of a 5 ms near-field stimulus. Data are means \pm 1 s.e.m. of 25 sensilla. The 0 degree position was directly ahead of (distal to) the sensillum under observation. The stimulus probe was moved sequentially in 20 degree increments to 17 different positions clockwise and counterclockwise around the sensillum. (Adapted from Mellon and Hamid [32]).

in the magnitude and/or direction of the perturbation can be detected by P. clarkii.

\subsubsection{Relative sensilla sensitivity and function}

Although the feathered sensilla was found to experience the largest force and bending moment, there are many scenarios and environmental conditions in which it would be advantageous to be less sensitive to fluid perturbations, including mechanosensing in high flow environments, mechanosensing during relatively fast antennule flicking, or mechanosensing by direct contact with solid surfaces $[7,8]$. These varied functions are performed by the range of sensilla types found on the antennules of crustaceans. The morphology and the fine structure of the aesthetasc sensilla in spiny lobsters and crayfish has been the subject of many studies [104, 105, 9]. During chemical stimulation, these organisms typically undergo a flicking 

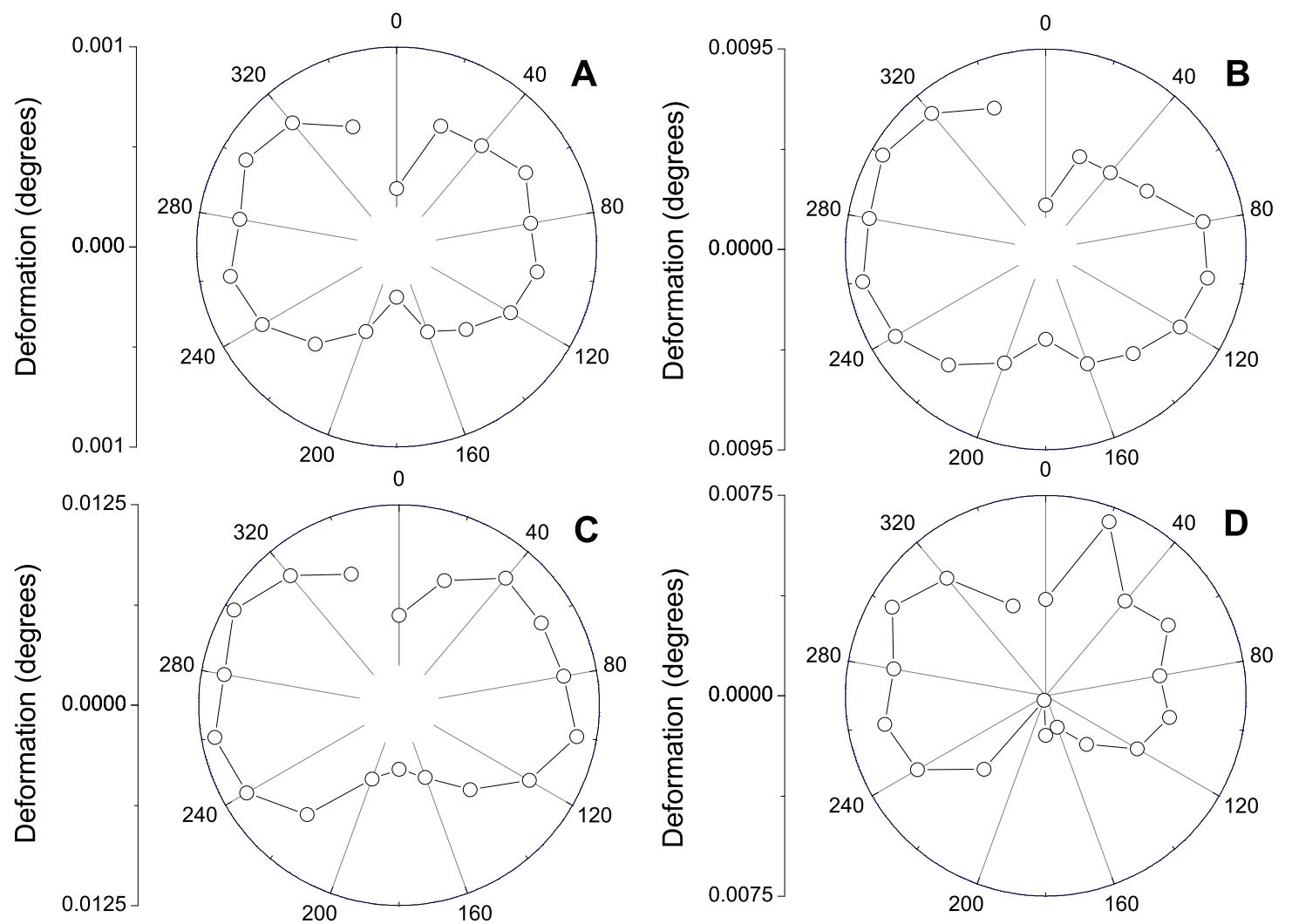

Figure 4.10: Angular deformation, $\delta$ of the sensilla at positions around the antennule for different morphologies: A. asymmetric, B. beaked, C. feathered and D. filamentous

response of their antennules, which initiates rapid antennule movement in the range between 1 $\mathrm{cm} \mathrm{s}^{-1}$ to $10 \mathrm{~cm} \mathrm{~s}^{-1}[11,10,29]$. Therefore, mechanosensors exposed to these rapid accelerations and fluid velocities, in addition to any surrounding ambient currents, must be able to detect and react to this fluid movement occurring at a much higher Reynolds number regime than the fluid perturbations studied. In contrast, there is also a need to detect and quickly respond to extremely small perturbations in the flow, often caused by predator attacks, and having multiple mechanosensor morphologies with varying sensitivities may play a fundamental role in enabling P. clarkii to detect hydrodynamic disturbances.

Of all the known morphologies on the crayfish antennule, the standing feathered sensilla is the most highly sensitive near-field hydrodynamic receptor with displacement firing threshold 


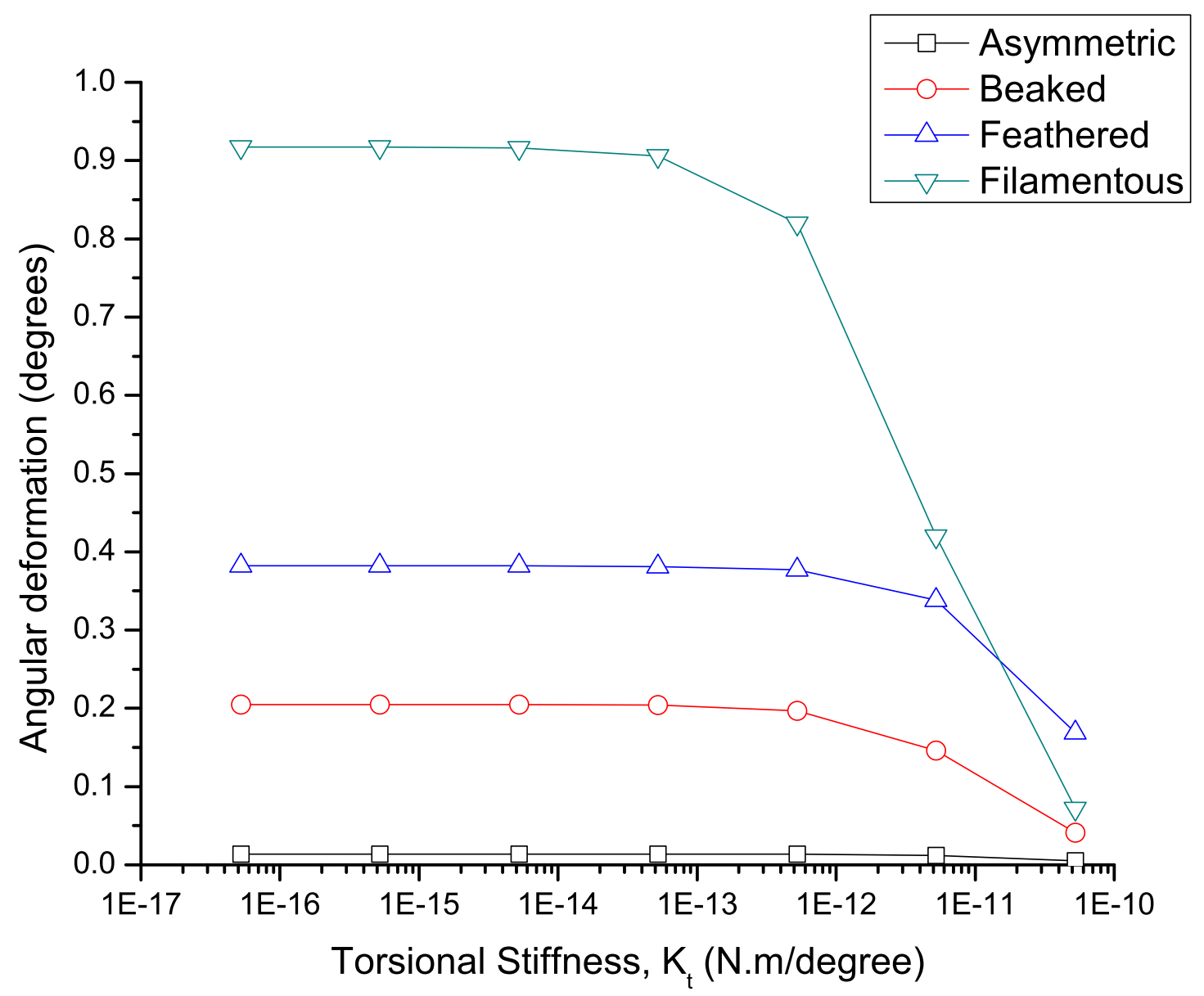

Figure 4.11: Sensitivity of sensilla deformation to variation in torsional stiffness, $k_{t}$ over several orders of magnitude. The AFM measurements and value used in the model are for $k_{t}=1 \times 10^{-12} \mathrm{~N} . \mathrm{m} /$ degree. The plot shows little variation in deformation $(\delta)$ for less stiff sensilla $\left(k_{t}<1 \times 10^{-12} \mathrm{~N} . \mathrm{m} /\right.$ degree$)$ but a sharp reduction in angular deformation, and thus sensitivity of the sensilla to hydrodynamic disturbance, for sensilla that have greater torsional stiffness.

as low as $0.02 \mu \mathrm{m}$ [7]. They are highly sensitive to hydrodynamic shear providing the first sign of warning for the animal to the possibility of the presence of a predator. The morphology of the sensilla, consisting of a dense array of branches, provides enhanced resistance to the incoming flow, increasing deformation of the sensilla structure at its joint. The beaked sensilla are the second most numerous morphology on the antennules of P. clarkii. Anatomical studies of similar sensilla in spiny lobsters suggest that these sensilla are bimodal contact 
chemo-mechanoreceptors $[98,99]$. Our results show that they have smaller deformational sensitivity (angular deflection in response to flow) than feathered sensilla. Their curved tip cuticle has been shown to have permeability to dye Crystal Violet [7], likely indicating they serve a function in chemical reception. These bimodal sensilla can initiate antennule flicking and owing to their lower hydrodynamic sensitivity, their mechanosensing function may be active during the flick to aid in chemical detection. The function of asymmetric and filamentous setae in P. clarkii in not completely established. The asymmetric sensilla have been inferred to be bimodal chemo-mechanoreceptor in lobsters [99]. They show the least sensitivity to flow-field perturbation in out results. Although their function is speculative, their low sensitivity allows them to respond either to fast water movements, such as during a flick, or act as contact sensilla. Filamentous setae are present in sparse population on the antennules on P. clarkii. These sensilla are the longest in length among all the sensilla morphologies tested in this study and have a group of filamentous hairs near its tip, which provides a long moment arm and hence a large angular deflection. Likely, their primary function is hydrodynamic sensing, as suggested earlier by Mellon [7], but it is unknown if they aid in chemosensing. Although the ultimate functioning of these various morphologies is unknown, it is likely that they all serve specific functions in the detection of their surrounding fluid environment. Further research in electrophysiology and brain response of the animals to stimulation of these sensilla, combined with numerical models that can elucidate the magnitude of perturbation required for hydrodynamic detection, can help achieve a greater understanding of their role in detecting hydrodynamic and chemical stimuli.

\subsubsection{Role of hydrodynamic sensing in plume tracking}

In aquatic benthic environments, flow fields are often turbulent and the distribution of diffusing odorants is intermittent and complex. The interwoven spatial and temporal structure of the flow and chemical distribution indicates that instantaneous sensing of the flow field can be of crucial importance in chemical sensing [95]. Antennule flicking enhances the 
detection of odorants by allowing the animal to take discrete samples of odorant molecules in addition to shedding away the boundary layer of odorants previously present around the sensilla. This enhances the odorant diffusion to the sensilla surface [12] and allows it to enter the permeable cuticle on the aesthetasc surface before it comes in contact with the ORN dendrites. It has been shown that flow and chemical signal integration in the form of odorant flux can enhance the animals ability to locate the source of a chemical plume [91]. Results from Pravin and Reidenbach [95] have shown that mean odorant concentration and intermittency in the odorant signal increase towards the plume source, but the temporal and spatial rate of this increase is slow. Long measurement times would be necessary to be useful for chemosensory guidance if odorant concentration were used alone. However, odorant fluxes measured transverse to the mean flow direction, measured as the simultaneous instantaneous fluctuation in concentration and velocity, have been shown to have statistically distinct magnitude and directional information. This information is spatially distinct on either side of a plume centerline, and can be determined with sampling times of $0.5 \mathrm{~s}$ or less. Aquatic animals typically have neural responses to odorant and velocity fields at rates between 50 and $500 \mathrm{~ms}$, suggesting this simultaneous sampling of both flow and concentration can be highly beneficial for rapid detection and movement towards the source of the odorant plume.

\subsubsection{Sensitivity to direction of perturbation}

In aquatic benthic environments, flow fields are often turbulent and the distribution of diffusing odorants is intermittent and complex. The interwoven spatial and temporal structure of the flow and chemical distribution indicates that instantaneous sensing of the flow field can be of crucial importance in chemical sensing [95]. Antennule flicking enhances the odorant diffusion to the sensilla surface [12] and allows it to enter the permeable cuticle on the aesthetasc surface before it comes in contact with the ORN dendrites. Although it has been shown that flow and chemical signal integration in the form of odorant flux can 
enhance the animals ability to locate the source of a chemical plume [91], how and to what extent an animal integrates hydrodynamic and chemical information is currently unknown. Results from Pravin and Reidenbach [95] have shown that mean odorant concentration and intermittency in the odorant signal increase towards the plume source, but the temporal and spatial rate of this increase is slow. Long measurement times would be necessary to be useful for chemosensory guidance if odorant concentration were used alone. However, odorant fluxes measured transverse to the mean flow direction, measured as the simultaneous instantaneous fluctuation in concentration and velocity, have been shown to have statistically distinct magnitude and directional information. This information is spatially distinct on either side of a plume centerline, and can be determined with sampling times of $0.5 \mathrm{~s}$ or less. Aquatic animals typically have neural responses to odorant and velocity fields at rates between 50 and $500 \mathrm{~ms}[62,66,58,106]$, suggesting this simultaneous sampling of both flow and concentration can be highly beneficial for rapid detection and movement towards the source of the odorant plume. Our findings suggest that due to the varying sensitivity of the mechano-sensilla to angle of hydrodynamic perturbation, that these sensilla can be used to determine what direction a flow disturbance originated from. As chemical signals become entrained by turbulence and mixed within the plume as they propagate downstream, sensing both odor concentration and direction from which these flavored eddies [34] originated from, can greatly enhance information input about source location. Mellon and Hamid [32] reported that the differences in directional sensitivities were not significant, except for a definite drop close to 180 degrees opposite the preferred direction. However, through an ANOVA analysis of the data, they showed highly significant differences in response threshold at different angles with reference to the sensilla $(\mathrm{P}<0.001)$. The results shown in this numerical study indicate slightly increased angular deflection of the sensilla for perturbations at right angles to the sensilla orientation and also a drop in deformation at 180 degrees to the preferred direction. This indicates that the sensilla although they are sensitive to direction of perturbation, retain their sensitivity from almost all directions except at $\theta=180^{\circ}$, when the flow movement occurs 
directly behind the sensilla orientation. In conclusion, a rich diversity of morphology is found among sensilla on the antennule of a crustacean. These morphologic variations allow it to detect both small and large perturbations under different flow conditions and, combined with chemoreceptor aesthetascs, provide the ability for integration of flow and chemical stimulus on relevant time and spatial scales to effectively navigate their benthic environment. 


\section{CHAPTER 5}

\section{Conclusions}

In this dissertation, I sought to answer three main questions about the chemical sensing mechanism in aquatic animals and the turbulent plume structure in benthic environment. These three questions are once again posed, and summaries of their answers are provided:

1. How does the morphology of aesthetasc sensilla and flicking kinematics effect odorant transport to the surface of the aesthetascs?

Results from numerical model of aesthetasc sensilla show that the rapid downstroke of the antennules facilitates transport of odorant molecules to the region surrounding the aesthetascs, while the slower return stroke enables for additional time for the entrapped odorants to molecularly diffuse to the aesthetasc surfaces. The quantity of odorant molecules present at the aesthetasc surface reaches peak values near the end of downstroke, but due to the comparatively slow nature of the return stroke, significant concentrations of odorant are retained in the vicinity of the aesthetascs at the end of the flick/return sequence. Subsequent

flicks were shown to remove the odorants captured during the previous flick and capture new odorants from the ambient flow, at a replacement rate of up to $97.6 \%$. The concentration of molecules captured along aesthetasc surfaces was found to increase with increased gap spacing between aesthetascs, flick speed, and distance from the proximal end of the aesthetasc, but these changes in morphology and flicking kinematics reduce the animals ability to take 
discrete samples of the odorant-laden fluid environment with each flick. Results suggest that antennule flicking allows discrete sampling of the time- and space-varying odorant signal, and high concentration odorant filaments can be distinguished from more diffuse, low concentration filaments through changes in both the timing and the encounter rate of odorant molecules to aesthetasc surfaces.

2. What is the impact of ambient flow environments on turbulent mixing and odorant transport within a chemical plume and can the animal conceivably integrate velocity and concentration information to aid in finding the location of the plume source?

Results shows that simultaneous sampling of both flow and odorant concentration can provide valuable cues for tracking of odorant plumes compared to sampling of odorants alone. Mean odorant concentration increases as the animal moves towards the plume source, helping it determine the upstream direction. Variability in the temporal rate of change of concentration also increases as the animal moves towards the source. However, both these statistics converge slowly, and therefore may not be useful for aquatic organisms that often make rapid movements while navigating in a plume [8]. Comparison of flux of odorants in the transverse direction between two points on either side of the plume centerline separates statistically on timescales $\leq 0.5 \mathrm{~s}$, while velocity and concentration tend to converge over similar lengths of time. This difference in flux becomes larger, and converges towards the plume centerline as the animal moves closer to the plume source. Although it is still unknown if crustaceans can integrate variations in odorant concentrations and velocity relative to background levels, our results suggest that flux can be a significant factor enabling animals to orient themselves favorably in the search for a plume source, and occurs on timescales relevant to responses by olfactory neurons. In addition, our results show that transverse flux is a useful parameter to be quantified and incorporated into the design and utilization of artificial bio-mimetic sensors. 
3. How does hydrodynamic perturbation effect flow and pressure distribution near an organism and what is the effect of sensilla morphology on their relative sensitivity to upstream perturbations?

Results show that the upstream motion causes alterations in flow velocity near the sensilla accompanied by corresponding variations in pressure. The standing feathered and filamentous sensilla experience more force, bending moment and deformation around their joint than beaked and asymmetric sensilla morphologies. The sensitivity of the sensilla correlates closely to their sensory function with the feathered and filamentous sensilla (high deformational sensitivity) acting as hydrodynamic receptors while beaked and asymmetric sensilla (low deformational sensitivity) acting as bimodal chemo-mechanoreceptors. A study of directional dependence of sensilla sensitivity, which quantifies the deformation of sensilla as a function of the direction of hydrodynamic perturbation relative to the sensilla orientation, shows comparable sensitivity at all angles except at 180 degrees where a sharp drop in sensitivity in observed. 
Appendices 


\title{
Appendix A
}

\section{A nose too far: regional differences in olfactory receptor neuron efficacy along the crayfish antennule}

This chapter has been published, with citation: Mellon DeF., Pravin S. , and Reidenbach M.A., (2014), "A nose too far: regional differences in olfactory receptor neuron efficacy along the crayfish antennule", Biological Bulletin 227:40-50.

\begin{abstract}
The olfactory sense organs of crayfish are aesthetasc sensilla, arrayed along the distal half of the lateral antennular flagella on each side of the animal. The sensillar array is sparse at its proximal origin, where each annulus houses only a single aesthetasc, and it is most dense distally, with occasionally up to six aesthetascs residing on each antennular annulus. Previous studies have tacitly assumed that the aesthetascs are co-equal in their functional properties. We restricted exposure of small zones of aesthetascs to odorant along the array, from near its proximal origin, its midpoint, and its termination near the tip of the lateral flagellum, while recording neural responses within the ipsilateral olfactory lobe of the brain.
\end{abstract}


Simultaneous combinations of zonal exposure to odorant gave proportionally larger central responses, indicative of spatial summation of peripheral inputs. Surprisingly, however, zonal effectiveness was not equal; stimulating even small numbers of aesthetascs near the proximal origin of the array was far more excitatory to local deutocerebral interneurons than stimulating greater numbers of aesthetascs at the tip of the flagellum. The results are discussed in terms of continuing growth and attrition of the antennular segmentation and associated olfactory receptor neurons.

\section{A.1 Introduction}

Capture of odorant stimuli by olfactory receptors in both vertebrates and invertebrates has received increasing attention since the initial cloning of mammalian genes expressing odorant receptor proteins in the early 1990s. The presence and distribution of receptor proteins on olfactory receptor neurons (ORNs) is well documented [107, 108, 109, 110, 111], and the patterns of central targeting by ORN axons have been successfully examined in both mammals and insects, where molecular genetic techniques have been especially compelling in demonstrating the specificity of anatomical relationships between identified olfactory glomeruli and the various ORN classes $[112,113,114,115,116]$. Other studies have focused upon the molecular structure of olfactory stimuli and, especially, the nature of signal transduction pathways leading to the generation of ORN spiking activity $[117,118,119]$

Much less is understood, however, about the numerical and functional relationships between ORNs and their individual target brain neurons. What are the biological constraints that dictate the most effective ratio of ORNs to their central neuronal targets? Does conjoint stimulation of individual ORNs by an odorant enhance the response of target brain neurons through simple summation, or is there non-linearity as the stimulated fraction of the homologous receptor array by the odorant increases? Finally, are all competent ORNs within the sensilla array equivalent in their individual responses to the same odorant? These and 
other questions related to the physical disposition and physiological relationships of ORNs on all metazoan sensory structures remain poorly understood.

In crustaceans, olfactory sensilla, referred to as aesthetascs, occur on the lateral flagellum of each antennule [120, 104, 105, 9], and each aesthetasc houses the distal dendrites of a variable number, usually 100-300, of olfactory receptor neurons (ORNs). Aesthetasc assemblages in many, if not most, crustaceans occur in comparatively short, dense tufts. This is the case with marine species such as palinurids, homarids, anomurids and the brachyura. In a few species, however, such as freshwater crayfishes and caridean prawns, the aesthetascs are rather sparsely arrayed linearly along the distal one half of the flagellum [104, 9, 121, 122]. The spatially distributed arrangement of aesthetasc sensilla in these animals provides an unusual opportunity to determine the extent to which fractional regions of the receptor array contribute to the central representation of odorants. Furthermore, crayfish antennular flagella grow continuously throughout an animals lifespan by adding annuli and sensilla at their base; this means that aesthetascs near the flagellum tip are the oldest, and in adult individuals a proportion of them, along with the distal annuli, are shed at each molt [123]. The linear distribution of sensilla, representing a spatial gradation in age, begs examination of possible age-related variations in odorant sensitivities of aesthetascs in different regions along the flagellar shaft. Moreover, because at least some local interneurons within the deutocerebrum receive short-latency inputs from hydrodynamic receptors on the antennular flagella in addition to long-latency inputs from the aesthetascs [68], interactions between the different central response phases may depend critically upon the transmission time to the brain from the receptor neurons in the different regions of the lateral flagellum. To address questions of spatial summation within the aesthetasc array and competency of ORNs along the array, we designed an olfactometer to effect regional stimulation of the aesthetasc array while recording electrical activity from OL interneurons in the brain of the crayfish Procambarus clarkii Girard. This apparatus permitted separate or conjoint stimulation by odorant at three different zones along the aesthetasc array: near the antennular flagellum tip 
(zone 3), in the middle of the array (zone 2), and just distal to the proximal origin of the array (zone 1), roughly half way between the flagellum base and the tip. We examined the responses of Type I local deutocerebral interneurons, broad-spectrum multiglomerular cells first characterized by Mellon and Alones [124] and which are driven by excitatory synaptic input from ipsilateral olfactory receptor neurons associated with the aesthetascs on the antennules, from twenty-two preparations to stimulation of different numbers of aesthetascs, including 14 cells from which we were able to determine a detailed differential response to zonal stimulation. The results of our studies permit us to conclude that odorant stimulation of a larger fraction of the sensilla array generally produces larger responses in OL neurons than smaller fractional stimulation. Furthermore, proximally-positioned aesthetascs generate dramatically more vigorous responses within the Type I neurons than those located at the middle or distal regions of the array. The stimulation techniques were verified both by using an active computer model of stimulus flow to examine the accuracy of the stimulus operation as well as by directly observing the stimulant flows restrictions exclusively to the three respective zones on the flagellum by means of planar laser-induced fluorescence (PLIF).

\section{A.2 Materials and Methods}

Animals used were large adult (50-60 mm carapace length) individuals of the southern red swamp crayfish Procambarus clarkii having antennules at least $25 \mathrm{~mm}$ in length. Crayfish were de-clawed and maintained in pairs in individual plastic tubs having approximately $3 \mathrm{~cm}$ of dechlorinated tap water, at room temperatures. Water was changed every other day. The crayfish were fed on frog chow and lettuce twice a week. For electrophysiological experiments, crayfish were chilled in crushed ice for approximately $30 \mathrm{~min}$ to anesthetize them. They were then decapitated by transverse cutting through the cephalothorax at a level just caudal to the base of the rostrum. The head capsule was rinsed in chilled crayfish saline (composition in mmol l-1: $\mathrm{NaCl}, 205 ; \mathrm{KCl}, 5.4 ; \mathrm{CaCl}_{2} .2 \mathrm{H}_{2} 0,13.6 ; \mathrm{MCl}_{2} .7 \mathrm{H}_{2} 0,2.7$; 
$\mathrm{NaHCO}_{3}, 2.4$ and the $\mathrm{pH}$ adjusted to 7.4 with $\mathrm{HCl}$ ), and the rostrum was removed. The isolated heads were mounted in a recording chamber, as described previously [68], except that all four antennular flagella were drawn by suction into the olfactometer diagrammed in Figure A.1(A) Diagram of the isolated head preparation and partitioned olfactometer used in the experimental procedures reported in this paper. The antennular flagella protrude through an aperture in the wall of the square recording chamber and into the olfactometer. Cannulas for brain perfusion and the recoding electrode placement are labelled "C". (B) detail of the partitioned olfactometer. Antennular flagella (not shown) protrude into the central horizontal chamber from the left and extend to the right beyond the most distal odorant port. Water enters the olfactometer chamber through the vertical port, while odorant enters through the three horizontal ports labeled "Odorant" and is removed via the ports labeled "Suction" after passing across the flagella.figure.caption.60 through a small opening in a Plexiglas partition. This provided a modicum of mechanical and, hence, positional stability to the flexible antennular flagella within the central channel of the olfactometer. Animals having discolored or broken aesthetascs were not used in this study. The olfactometer consisted of a central cylindrical channel, $25 \mathrm{~mm}$ in length and $1.2 \mathrm{~mm}$ in diameter, that was aligned with the opening in the recording chamber and which was open at the opposite end. The bases of the antennules were sealed within the olfactometer with petroleum jelly to isolate them from the recording chamber.

Three pairs of transversely aligned side channels, $1.1 \mathrm{~mm}$ in diameter, intersected the main channel at, respectively, $2.5 \mathrm{~mm}, 7.5 \mathrm{~mm}$ and $12 \mathrm{~mm}$ from the distal opening of the main olfactometer channel, as shown in Figure A.1(A) Diagram of the isolated head preparation and partitioned olfactometer used in the experimental procedures reported in this paper. The antennular flagella protrude through an aperture in the wall of the square recording chamber and into the olfactometer. Cannulas for brain perfusion and the recoding electrode placement are labelled "C". (B) detail of the partitioned olfactometer. Antennular flagella (not shown) protrude into the central horizontal chamber from the left and extend to the right 

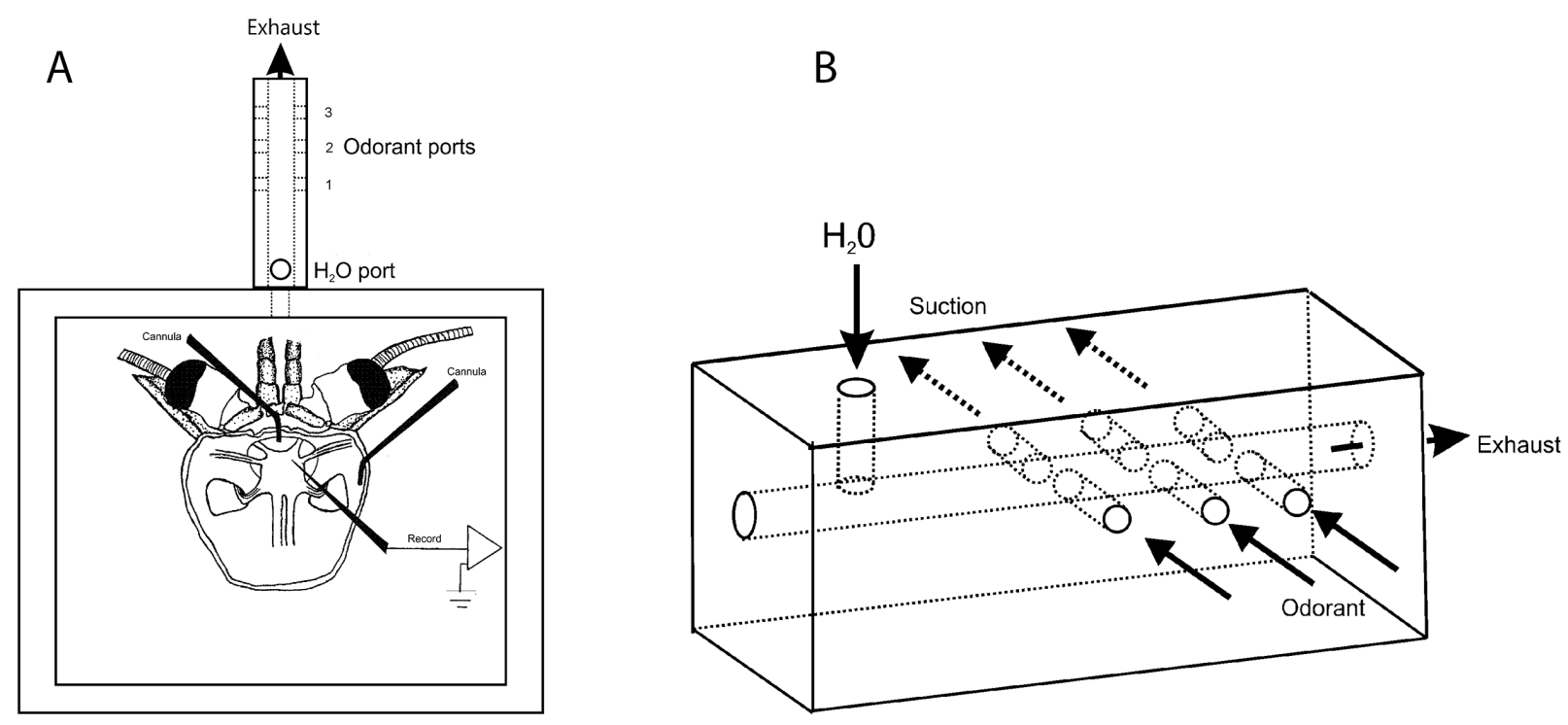

Figure A.1: (A) Diagram of the isolated head preparation and partitioned olfactometer used in the experimental procedures reported in this paper. The antennular flagella protrude through an aperture in the wall of the square recording chamber and into the olfactometer. Cannulas for brain perfusion and the recoding electrode placement are labelled "C". (B) detail of the partitioned olfactometer. Antennular flagella (not shown) protrude into the central horizontal chamber from the left and extend to the right beyond the most distal odorant port. Water enters the olfactometer chamber through the vertical port, while odorant enters through the three horizontal ports labeled "Odorant" and is removed via the ports labeled "Suction" after passing across the flagella.

beyond the most distal odorant port. Water enters the olfactometer chamber through the vertical port, while odorant enters through the three horizontal ports labeled "Odorant" and is removed via the ports labeled "Suction" after passing across the flagella.figure.caption.60B. On the top of the olfactometer near the recording chamber, a vertical cylindrical channel $1.2 \mathrm{~mm}$ in diameter intersected the main channel from above. The vertical channel provided access to a reservoir of dechlorinated tap water, which exited by gravity flow through the distal opening into a sump after flowing past the antennular flagella. The maximal flow rate of water through the central chamber when it was occupied by the four antennular flagella was $15.6 \mathrm{ml}$ per min. Each of the three side channels on one side could be individually connected by an electronically controlled solenoid switch to an odorant reservoir containing 
$.05 \%$ tetramin solution, made up by dissolving 1 gram of Tetramin fish food pellets in $100 \mathrm{ml}$ of dechlorinated tap water and diluting appropriately. The same electrical pulses used to control odorant access also opened the paired, opposite side channels to a vacuum source, so that, during stimulation from a port, odorant and reservoir water were drawn across the main channel, past the antennular flagella, and into the vacuum exhaust channel. Protocol for water and stimulus delivery at each zone were as follows: 1) Water onset; 2) odorant onset 2 seconds later for $0.5 \mathrm{sec} ; 3)$ water off 8 seconds later. As soon as the isolated head had been mounted in the recording chamber, the median (cerebral) artery and the lateral cephalic artery on one side of the head were cannulated and perfused with chilled $\left(16{ }^{\circ} \mathrm{C}\right)$ oxygenated crayfish saline at a flow rate of $2 \mathrm{ml} / \mathrm{min}$. The perineurium was removed from the dorsal surface of the brain to expose the OLs. Sharp micropipette electrodes filled with 2 M potassium acetate were used to impale the large dendritic trunks of Type I local interneurons within the OL. Type I interneurons are the largest neurons that have been characterized in the crayfish deutocerebrum [124] with dendritic processes up to $20 \mu \mathrm{m}$ in diameter within the OL; they can be more readily penetrated with sharp micropipette electrodes than any other resident interneurons. Signals from impaled neurons were led to an Axoclamp 2B amplifier (Molecular Devices, Sunnyvale, CA, USA), digitized, and stored in a computer file for later analysis. Solenoid switches (Lee Company, Westbrook, CT, USA) used to deliver water and the odorant pulses and to control access to suction to the three stimulus ports, were activated by Grass S48 stimulators (AstroMed, Warwick, RI, USA) that could be energized separately, sequentially, or simultaneously. Odorant delivery was gravity fed at $11 \mathrm{ml} / \mathrm{min}$. Responses to odorant pulses were recorded as the number of spikes during a 5 sec time window commencing 200 msec following odorant pulse onset (to avoid secondary hydrodynamic responses to fluid onset). Within each test series, sequential stimulation of specific ports and/or port combinations were varied randomly to obviate any possible effects of physiological deterioration of the preparation. Mean values for spike numbers in response to 2-13 repetitions - at 2-min intervals - of odorant pulses were computed and were divided by 
the number of aesthetasc sensilla within the stimulus column (= diameter of the stimulus port) at each zone to generate a response coefficient for that zone. In two preparations, only two or three observations in each of the seven stimulating situations were collected due to the loss of microelectrode penetration. After response sequences were obtained from a preparation, the isolated head was left in place in the recording chamber, and the margins of the three stimulating zones within the aesthetasc array along the antennular flagellum were carefully measured, in terms of the identity of included flagellar annuli, using a dissecting microscope. The measurements were then translated to their fractional positions (as percent of the linear distance between the proximal origin of the aesthetasc array and its termination at the flagellar tip) along a dimensionless variable axis (z) representing the array of aesthetascs. Finally, the number of aesthetascs within the boundaries of the identified annuli in each of the three stimulus zones were carefully counted and recorded using a compound microscope. The absence of odorant spread between the three stimulus ports lengthwise along the water channel was verified by visualizing the flow of odorant across the central channel from each of the supply ports to the corresponding suction-exhaust port. Planar laser induced fluorescence (PLIF) was thereby used to verify that odorant flow through each of the pairs of side channels was confined to that pair of channels alone during actual tests with antennules from moribund crayfish heads identical in size to those used in the experiments with living material. This technique used fluorescein dye (molecular diffusion in water, $D=0.5 \times 10^{-9} \mathrm{~m}^{2} \mathrm{~s}^{-1}$ ) as the scalar tracer, while a $488 \mathrm{~nm}$ laser was used to excite the fluorescein (mean excitation at 490 $\mathrm{nm}$ and mean emission at $520 \mathrm{~nm}$ ). The laser beam was first passed through a beam focus and then aligned to illuminate only the antennule chamber by directing the laser beam into the exhaust port of the chamber. Fluorescein dye was added independently into each of the three stimulus ports and injected into the main chamber. A digital camera (1Mpixel, 12 bit resolution) (Dalsa 1M60, Waterloo, Ontario, Canada), imaged the fluoresced light through the top of the chamber which was optically transparent. The camera was fitted with a longpass filter, which passed all light above $517 \mathrm{~nm}$ from the excited dye, while ambient laser light 
was blocked. Light intensity emitted from the fluorescein dye is directly proportional to dye concentration, and a linear correlation between light emission and dye concentration was formed. The aperture of the camera was adjusted such that the source concentration of the dye injected into the chamber corresponded to near saturation levels of light into the camera. Since the camera has 12 bit resolution, concentrations could be quantified down to 1/4096 of the source concentration. Figure A.2Planar laser induced fluorescence images of dye within the central cylindrical chamber (denoted by dashed horizontal lines) where the antennule is located, sequentially applied through each of the three transversely aligned side channels (denoted by the dashed vertical lines). Concentration is normalized by input concentration and shows little mixing within the central chamber.figure.caption.61 shows the colormap of dye concentration within the chamber during separate, sequential dye releases within each port, which closely matches the numerical simulation. This figure also shows that dye does not escape out of the exhaust port of the chamber.

\section{A.3 Results}

\section{A.3.1 Antennular anatomy and aesthetasc distribution}

Figure A.4Distribution of aesthetasc number per annulus along the array in four freshly excised lateral antennular flagella (A and B are from opposite sides of the same crayfish, C and $\mathrm{D}$ are from two different crayfish) from animals in the range of 55-60 mm carapace length. The sensilla arrays, occupying between 32 and 42 flagellar annuli, are represented as dimensionless variables, $z$, where 0 is at the origin of the array, roughly half way distal from the base of the flagellum, and 1 is the termination of the array at the tip. The vertical rectangles in A approximate the position of ports one, two and three with respect to the aesthetasc array. While there is considerable variability between individual flagella, it is clear that the most proximal annuli harbor the fewest aesthetascs and that the density of aesthetascs per annulus increases distally.figure.caption.63A is a phase contrast light micrograph of a portion of a 


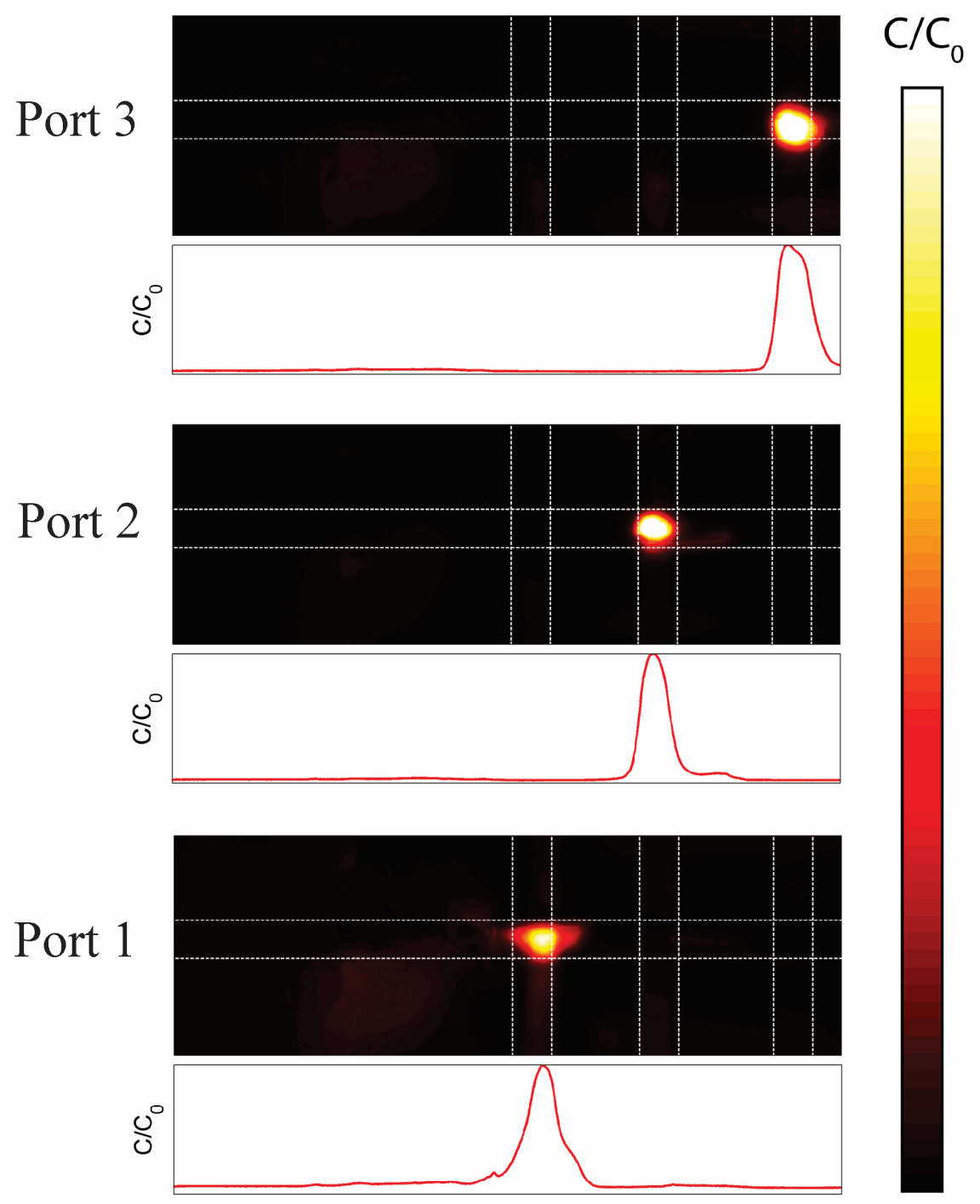

Figure A.2: Planar laser induced fluorescence images of dye within the central cylindrical chamber (denoted by dashed horizontal lines) where the antennule is located, sequentially applied through each of the three transversely aligned side channels (denoted by the dashed vertical lines). Concentration is normalized by input concentration and shows little mixing within the central chamber.

living lateral flagellum from P. clarkii. Aesthetasc sensilla (white arrows) are blunt setae roughly $100 \mu \mathrm{m}$ long, 10-15 $\mu \mathrm{m}$ in diameter at their base and tapering to a smaller diameter at their tip. The distal $40 \%$ of each aesthetasc has a thin $(1 \mu \mathrm{m}$ thick $)$ optically transparent cuticle that is apparently the region through which odorant molecules can pass to the interior 

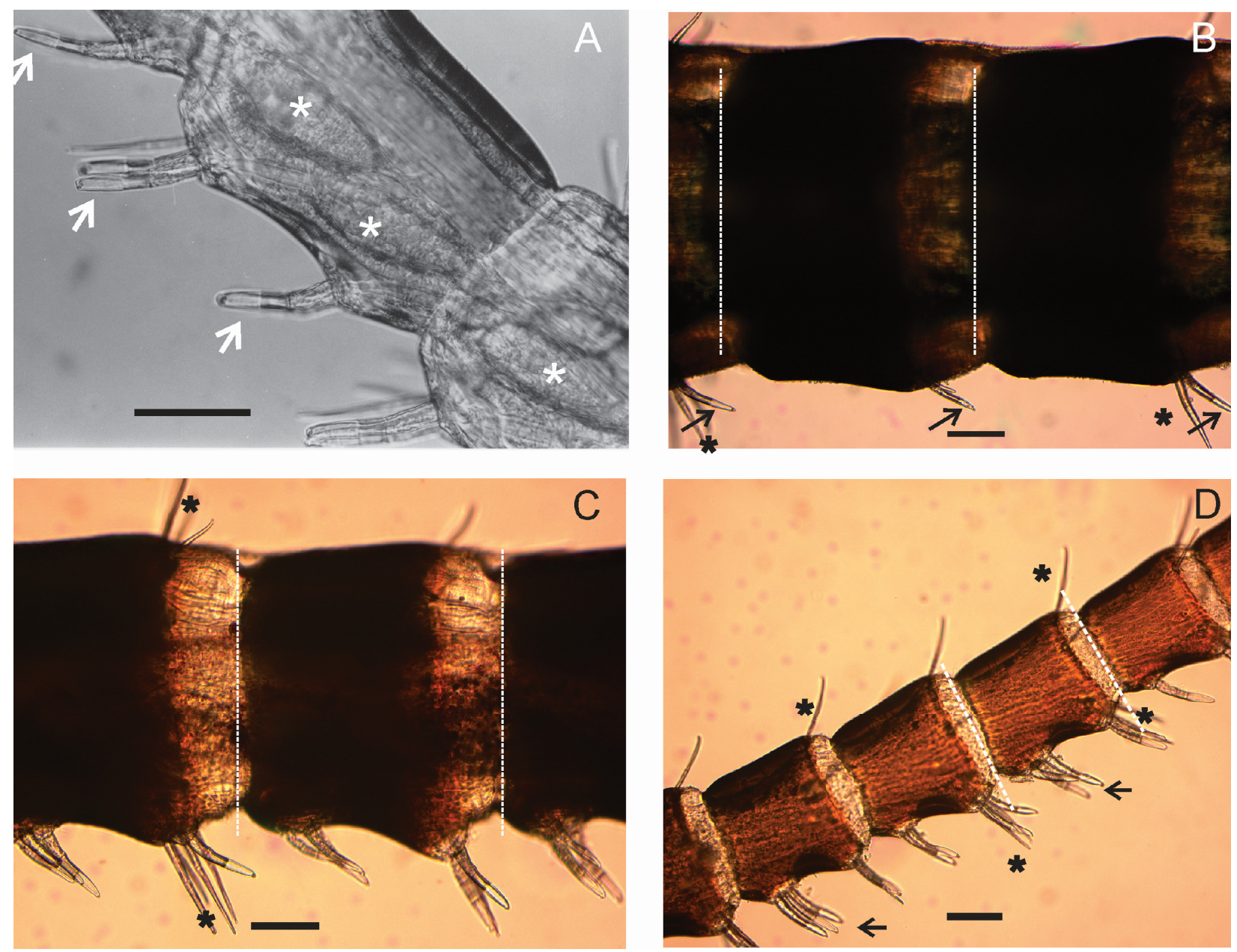

Figure A.3: A, phase contrast micrograph of portion of a living lateral antennular flagellum from P. clarkii, modified from Mellon et al [9]. Aesthetasc sensilla are indicated by white arrows; each aesthetasc is supplied by the distal dendritic segments from approximately 175 ORNs, the cell bodies of which are clustered in individual sensory ganglia that are partially visible (asterisks) through the flagellar cuticle. B, light micrograph of a portion of a freshly excised lateral antennular flagellum from P. clarkii. The two antennular annuli included in the image (annulus borders indicated by white dashed lines) are from a region just distal to the origin of the aesthetasc array; each annulus in this region generally bears just a single aesthetasc (black arrows). Additional, numerous setae seen in micrographs B-D (black asterisks) are beaked sensilla [7] believed to serve a bi-modal mechano-chemosensory function). C, micrograph from the same lateral flagellum as in B, but from a region about $40 \%$ of the total length of the array distal to the origin $(z \sim 0.4)$. Here, each annulus is smaller than those near the array origin, and each bears three to four aesthetasc sensilla. $\mathrm{D}$, a portion of the same flagellum near the tip; aesthetascs often occur in clusters of three (arrows) at the proximal edge of an annulus and two at the distal edge, for a total of four to five per annulus, each of which is only about one-third the size of those shown in $\mathrm{C}$ and which, therefore, present greater numbers of aesthetascs exposed to the odorant stimuli at the port. All scale bars are $100 \mu \mathrm{m}$. 
[104]. Each aesthetasc houses the distal dendrites of approximately 175 ORNs [9]; within the distal reaches of each aesthetasc the distal dendrites individually branch repeatedly into single microtubules, in which are imbedded olfactory receptor proteins. It is not known whether the number of ORNs associated with aesthetascs at different locations within the linear array is variable, nor if the number of functionally viable ORNs varies with the age of the aesthetascs.

In the crayfish, aesthetasc sensilla are arrayed along the ventral aspect of the distal one-half of the lateral antennular flagellum. Their axial density along the array varies dramatically from the origin of the array to its termination near the tip of the flagellum, with low densities at the origin compared to those at the tip. The differences in aesthetasc density can be appreciated from the light micrographs of regions of a lateral flagellum, freshly excised from a large adult P. clarkii, in Figure A.4Distribution of aesthetasc number per annulus along the array in four freshly excised lateral antennular flagella (A and B are from opposite sides of the same crayfish, C and D are from two different crayfish) from animals in the range of 55-60 mm carapace length. The sensilla arrays, occupying between 32 and 42 flagellar annuli, are represented as dimensionless variables, $z$, where 0 is at the origin of the array, roughly half way distal from the base of the flagellum, and 1 is the termination of the array at the tip. The vertical rectangles in A approximate the position of ports one, two and three with respect to the aesthetasc array. While there is considerable variability between individual flagella, it is clear that the most proximal annuli harbor the fewest aesthetascs and that the density of aesthetascs per annulus increases distally.figure.caption.63, B-D, showing sample annuli from (B) the region near the origin of the aesthetasc array, (C) a region close to the center of the array, and (D) a region just proximal to the tip of the flagellum, the distal termination of the array. At the arrays origin, aesthetasc density per annulus varies from 1 per annulus (or 1 every other annulus) to two per annulus. In the center of the array the density is usually 3-4 per annulus, whereas near the tip the density is 4-5 per annulus. These changes are graphically illustrated in Figure A.5Electrical records from a Type I neuron 

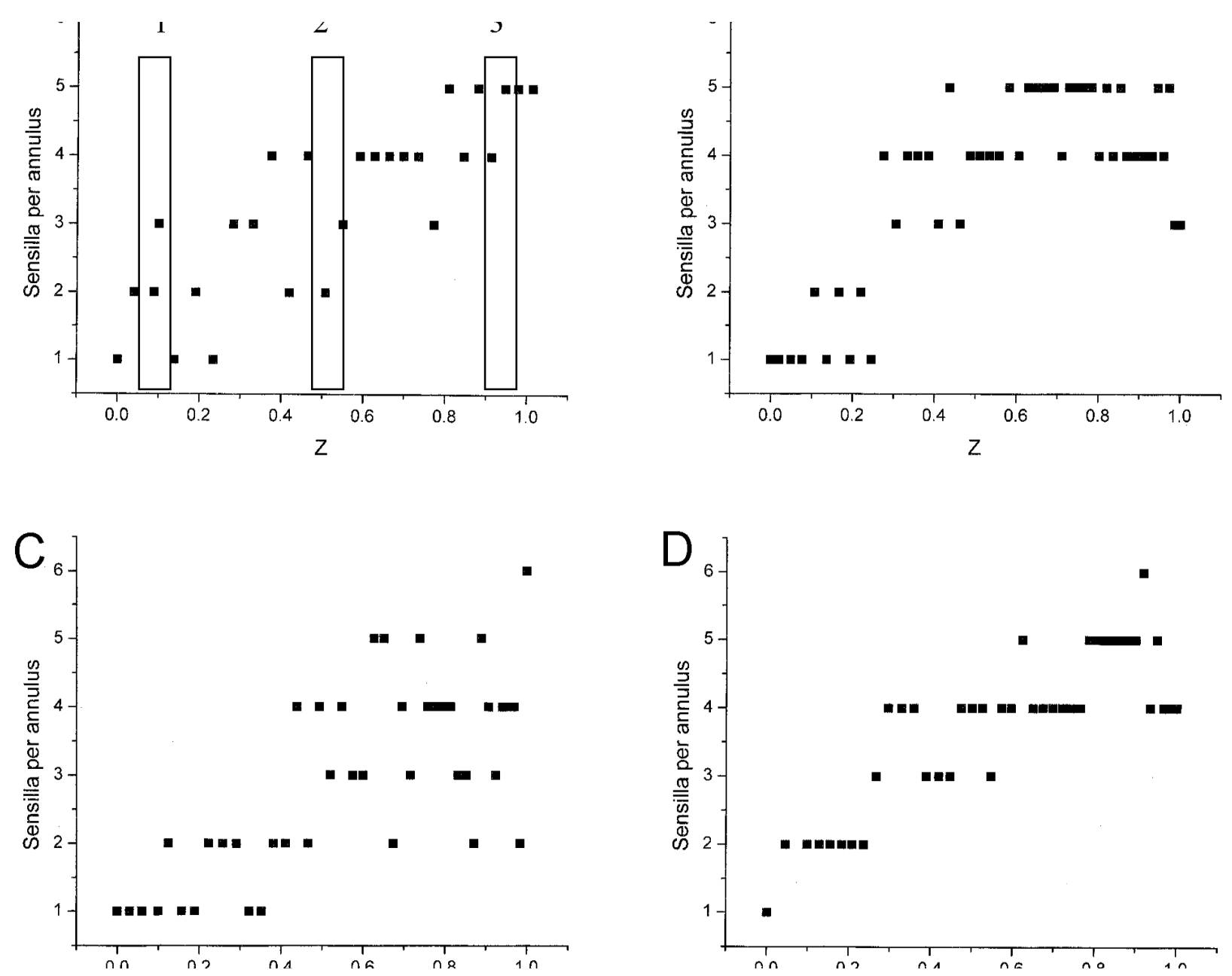

Figure A.4: Distribution of aesthetasc number per annulus along the array in four freshly excised lateral antennular flagella (A and B are from opposite sides of the same crayfish, $\mathrm{C}$ and $\mathrm{D}$ are from two different crayfish) from animals in the range of 55-60 mm carapace length. The sensilla arrays, occupying between 32 and 42 flagellar annuli, are represented as dimensionless variables, $z$, where 0 is at the origin of the array, roughly half way distal from the base of the flagellum, and 1 is the termination of the array at the tip. The vertical rectangles in $\mathrm{A}$ approximate the position of ports one, two and three with respect to the aesthetasc array. While there is considerable variability between individual flagella, it is clear that the most proximal annuli harbor the fewest aesthetascs and that the density of aesthetascs per annulus increases distally. 
exemplifying responses to zonal exposure of the lateral flagellum to odorant. A-G, responses to $0.5 \mathrm{sec}$ odorant pulses (solid bars beneath electrical records) via the indicated ports or port combinations, occurring $2 \mathrm{sec}$ following the onset of water through the central olfactometer chamber). The gray rectangle delineates initial spikes generated by hydrodynamic inputs from the flagellum at the onset of water flow [68]. The number of aesthetascs exposed to odorant at port 1 was 1 , the number exposed at port 2 was 16 , and the number exposed at port 3 was 17 . The $z$ position of the aesthetasc array at port 1 was 0 , that is, it was at the initial annulus of the array. Comparing the records in D-G with those in A-C, there is a clear indication of the additive relationship between central response and the extent of peripheral stimulation.figure.caption.64, showing the aesthetasc distribution along the array, proximal-to-distal, in four lateral flagella taken from three different animals of about $55 \mathrm{~mm}$ in carapace length. The array is usually about $13-15 \mathrm{~mm}$ in length in crayfish of this size, but here in each case the array has been converted to a dimensionless variable, $z$, that varies between 0 (at the origin) and 1 (at the tip). The vertical rectangles in Figure A.4Distribution of aesthetasc number per annulus along the array in four freshly excised lateral antennular flagella (A and B are from opposite sides of the same crayfish, C and D are from two different crayfish) from animals in the range of $55-60 \mathrm{~mm}$ carapace length. The sensilla arrays, occupying between 32 and 42 flagellar annuli, are represented as dimensionless variables, $z$, where 0 is at the origin of the array, roughly half way distal from the base of the flagellum, and 1 is the termination of the array at the tip. The vertical rectangles in A approximate the position of ports one, two and three with respect to the aesthetasc array. While there is considerable variability between individual flagella, it is clear that the most proximal annuli harbor the fewest aesthetascs and that the density of aesthetascs per annulus increases distally.figure.caption.63A, labeled 1-3, indicate the approximate extent and position of the stimulus ports within a typical aesthetasc array. 


\section{A.3.2 Neurophysiological observations}

Initial studies with several perfused isolated head preparations were designed to examine the role of individual groups of aesthetascs regardless of their position along the lateral flagellum array. Experiments were performed using the olfactometer illustrated in Figure A.1(A) Diagram of the isolated head preparation and partitioned olfactometer used in the experimental procedures reported in this paper. The antennular flagella protrude through an aperture in the wall of the square recording chamber and into the olfactometer. Cannulas for brain perfusion and the recoding electrode placement are labelled "C". (B) detail of the partitioned olfactometer. Antennular flagella (not shown) protrude into the central horizontal chamber from the left and extend to the right beyond the most distal odorant port. Water enters the olfactometer chamber through the vertical port, while odorant enters through the three horizontal ports labeled "Odorant" and is removed via the ports labeled "Suction" after passing across the flagella.figure.caption.60, having three approximately equally-spaced odorant ports operated separately or in different simultaneous combinations to stimulate the aesthetasc zones, i.e., zones $1,2,3,1+2,1+3,2+3$, or $1+2+3$. As described above and graphically in Figure A.4Distribution of aesthetasc number per annulus along the array in four freshly excised lateral antennular flagella (A and B are from opposite sides of the same crayfish, $\mathrm{C}$ and $\mathrm{D}$ are from two different crayfish) from animals in the range of 55-60 mm carapace length. The sensilla arrays, occupying between 32 and 42 flagellar annuli, are represented as dimensionless variables, $z$, where 0 is at the origin of the array, roughly half way distal from the base of the flagellum, and 1 is the termination of the array at the tip. The vertical rectangles in A approximate the position of ports one, two and three with respect to the aesthetasc array. While there is considerable variability between individual flagella, it is clear that the most proximal annuli harbor the fewest aesthetascs and that the density of aesthetascs per annulus increases distally.figure.caption.63, in an adult P. clarkii, the density of aesthetascs per antennular segment (annulus) varies from 0.5-2.0 near the proximal origin of the array to 4-6 near the distal termination of the array. The electrophysiological results 
from one isolated head preparation are shown in the records of Figure A.5Electrical records from a Type I neuron exemplifying responses to zonal exposure of the lateral flagellum to odorant. A-G, responses to $0.5 \mathrm{sec}$ odorant pulses (solid bars beneath electrical records) via the indicated ports or port combinations, occurring $2 \mathrm{sec}$ following the onset of water through the central olfactometer chamber). The gray rectangle delineates initial spikes generated by hydrodynamic inputs from the flagellum at the onset of water flow [68]. The number of aesthetascs exposed to odorant at port 1 was 1 , the number exposed at port 2 was 16 , and the number exposed at port 3 was 17 . The $z$ position of the aesthetasc array at port 1 was 0, that is, it was at the initial annulus of the array. Comparing the records in D-G with those in $\mathrm{A}-\mathrm{C}$, there is a clear indication of the additive relationship between central response and the extent of peripheral stimulation.figure.caption.64. They show spiking responses from a Type I neuron to $0.5 \mathrm{sec}$ applications of tetramin to zone 3 in $\mathrm{A}$, zone 2 in $\mathrm{B}$, zone 1 in $\mathrm{C}$, and various combinations of zonal stimulus delivery in D-G. The concentration of odorant stimulus, its timing following water onset, and its duration were identical in A-C, although the spike number and mean frequency varied depending upon the stimulation zone. Surprisingly, the smallest response occurred to stimulation of port 3, an area of the flagellum where the density of aesthetasc sensilla is at its greatest, and the highest response was obtained following stimulation via port 1 , where the aesthetasc density is very low. The results do show, however, that increasing the number of ports through which odorant was simultaneously applied increased the spiking response significantly: the response in D was obtained to simultaneous exposure of zones 2 and 3, that in E to exposure of zones 1 and 3, in $\mathrm{F}$ to simultaneous exposure of zones 1 and 2, and in $\mathrm{G}$ to exposure of all three ports together.

Data from three different preparations are shown in Figure A.6Spiking responses of three Type I interneurons to different numbers of stimulated aesthetascs, including those exposed by stimulus pulses in ports $1,2,3$ and $1+2,1+3,2+3$ and $1+2+3$. The responses of individual Type I cells are indicated by the different colored symbols. The regression 


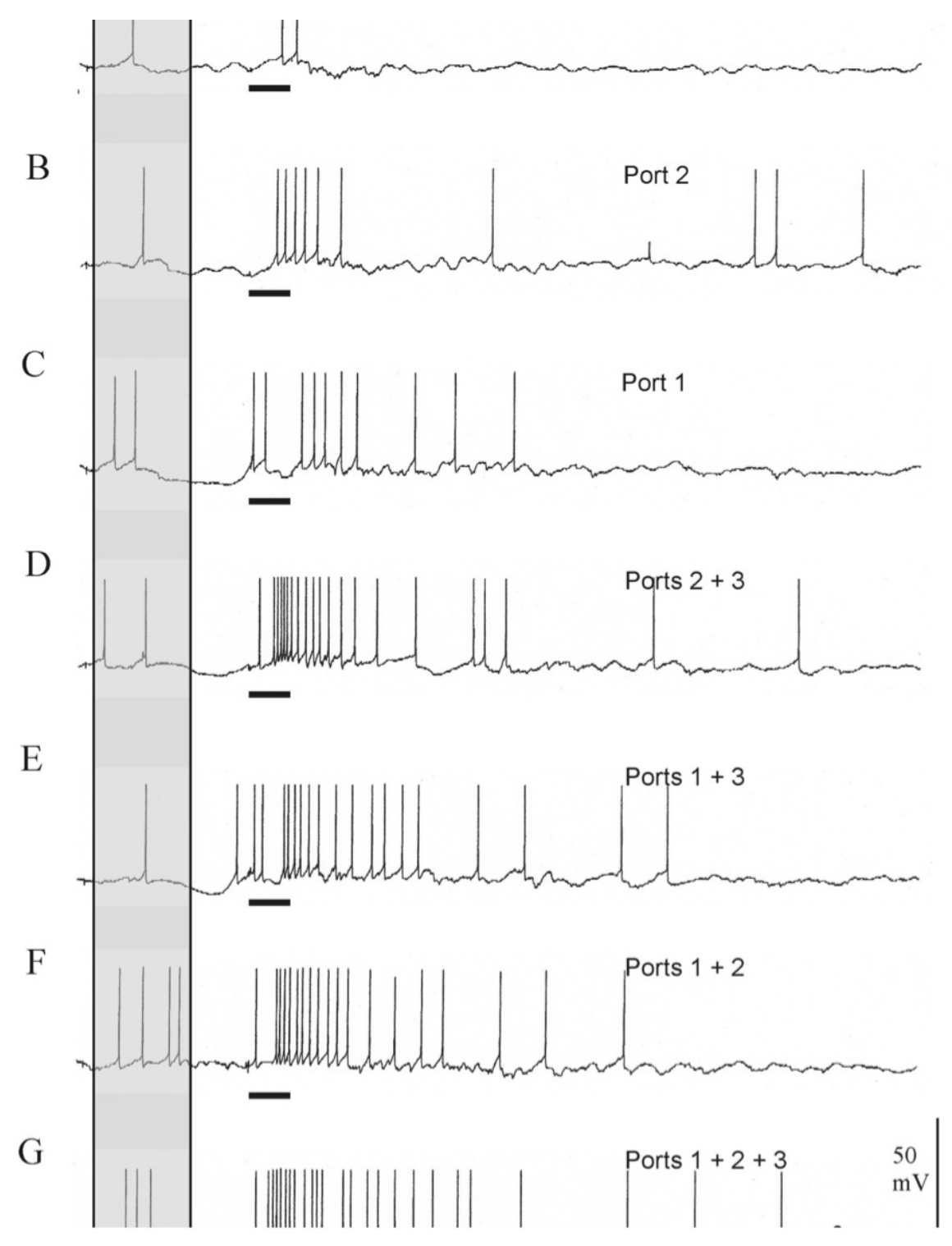

Figure A.5: Electrical records from a Type I neuron exemplifying responses to zonal exposure of the lateral flagellum to odorant. A-G, responses to $0.5 \mathrm{sec}$ odorant pulses (solid bars beneath electrical records) via the indicated ports or port combinations, occurring $2 \mathrm{sec}$ following the onset of water through the central olfactometer chamber). The gray rectangle delineates initial spikes generated by hydrodynamic inputs from the flagellum at the onset of water flow [68]. The number of aesthetascs exposed to odorant at port 1 was 1 , the number exposed at port 2 was 16, and the number exposed at port 3 was 17 . The $z$ position of the aesthetasc array at port 1 was 0 , that is, it was at the initial annulus of the array. Comparing the records in D-G with those in A-C, there is a clear indication of the additive relationship between central response and the extent of peripheral stimulation. 
curve represents best linear fit of the combined data; it was calculated by least squares regression analysis and is represented by the equation $y=0.41 x+18.57$. Pearsons $r$ is 0.87 and $\mathrm{P}>0.0001$.figure.caption.66; they are graphed together and illustrate the typical relationship between the response magnitude and the number of aesthetascs simultaneously stimulated. The relationship is highly statistically significant. Results from an additional five preparations are shown in Table A.1Spiking responses to zone - and zonal combination stimulation by odorant. Mean spike numbers (APs) \pm 1 S.E. (SE) in response to stimulation of different numbers of aesthetascs (Aes) occurring within single zones or zonal combinations in five preparations, identified by bold-numbered dates above the columns, in addition to those graphed in Figure A.7(A) Mean response coefficients obtained from Type I local olfactory interneurons in fourteen preparations to separate stimulation via the three stimulus ports. Zone one, nearest the proximal origin of the aesthetasc array, consistently elicited the most vigorous responses to standard odorant exposures. (B) Graph of response coefficients in local olfactory interneurons from the same fourteen different preparations against Z, a dimensionless variable which varies between 0 and 1 as a function of distance along the aesthetasc array from its origin to its termination at the flagellum tip. Responses from each preparation are represented here by a different colored symbol; data points in most cases are the mean of 5 -10 stimulus trials \pm 1 S.E. The data are satisfactorily fit by a first-order exponential decay curve defined by the equation $Y=6.67 e^{-X / .21}+0.27$. Nonlinear regression analysis on this functional model yielded $r^{2}=0.6$ and $\mathrm{P}<0.0001$.figure.caption.67. Pearsons $\mathrm{r}$ values were obtained by linear regression analysis. Values of $\mathrm{P}$ were calculated using software at www.danielsoper.com/statcalc3/calc.aspx?id=44.table.caption.65. The mean spiking responses from each preparation are based upon from 2 to 13 measurements in each of the seven stimulus conditions described above, in which the number of aesthetascs exposed in each condition were counted. Regression analysis was used to analyze the relationships in Table A.1Spiking responses to zone - and zonal combination stimulation by odorant. Mean spike numbers $(\mathrm{APs}) \pm 1$ S.E. (SE) in response to stimulation of different numbers of 
aesthetascs (Aes) occurring within single zones or zonal combinations in five preparations, identified by bold-numbered dates above the columns, in addition to those graphed in Figure A.7(A) Mean response coefficients obtained from Type I local olfactory interneurons in fourteen preparations to separate stimulation via the three stimulus ports. Zone one, nearest the proximal origin of the aesthetasc array, consistently elicited the most vigorous responses to standard odorant exposures. (B) Graph of response coefficients in local olfactory interneurons from the same fourteen different preparations against Z, a dimensionless variable which varies between 0 and 1 as a function of distance along the aesthetasc array from its origin to its termination at the flagellum tip. Responses from each preparation are represented here by a different colored symbol; data points in most cases are the mean of 5-10 stimulus trials \pm 1 S.E. The data are satisfactorily fit by a first-order exponential decay curve defined by the equation $Y=6.67 e^{-X / .21}+0.27$. Nonlinear regression analysis on this functional model yielded $r^{2}=0.6$ and $\mathrm{P}<0.0001$.figure.caption.67. Pearsons $\mathrm{r}$ values were obtained by linear regression analysis. Values of $\mathrm{P}$ were calculated using software at www.danielsoper.com/statcalc3/calc.aspx?id=44.table.caption.65; only the data from 5/24/12 were not statistically significant at the 0.05 probability level or less. Both Figure A.6Spiking responses of three Type I interneurons to different numbers of stimulated aesthetascs, including those exposed by stimulus pulses in ports $1,2,3$ and $1+2,1+3,2+3$ and $1+2+3$. The responses of individual Type I cells are indicated by the different colored symbols. The regression curve represents best linear fit of the combined data; it was calculated by least squares regression analysis and is represented by the equation $y=0.41 x+18.57$. Pearsons $r$ is 0.87 and $\mathrm{P}>0.0001$.figure.caption.66 and Table A.1Spiking responses to zone - and zonal combination stimulation by odorant. Mean spike numbers (APs) \pm 1 S.E. (SE) in response to stimulation of different numbers of aesthetascs (Aes) occurring within single zones or zonal combinations in five preparations, identified by bold-numbered dates above the columns, in addition to those graphed in Figure A.7(A) Mean response coefficients obtained from Type I local olfactory interneurons in fourteen preparations to separate stimulation via the three 
stimulus ports. Zone one, nearest the proximal origin of the aesthetasc array, consistently elicited the most vigorous responses to standard odorant exposures. (B) Graph of response coefficients in local olfactory interneurons from the same fourteen different preparations against Z, a dimensionless variable which varies between 0 and 1 as a function of distance along the aesthetasc array from its origin to its termination at the flagellum tip. Responses from each preparation are represented here by a different colored symbol; data points in most cases are the mean of $5-10$ stimulus trials \pm 1 S.E. The data are satisfactorily fit by a first-order exponential decay curve defined by the equation $Y=6.67 e^{-X / .21}+0.27$. Nonlinear regression analysis on this functional model yielded $r^{2}=0.6$ and $\mathrm{P}<0.0001$.figure.caption.67. Pearsons $\mathrm{r}$ values were obtained by linear regression analysis. Values of $\mathrm{P}$ were calculated using software at www.danielsoper.com/statcalc3/calc.aspx?id=44.table.caption.65 indicate that the responses from each Type I cell in the brain are additive and depend upon the net increment in the number of aesthetascs simultaneously exposed to a standard odorant pulse. A detailed examination of these data also suggested, however, that there was considerable variance in the overall effectiveness of different zonal stimulus combinations, with larger aesthetasc numbers at the distal part of the flagellum being much less effective than similar or even lesser numbers of aesthetascs from the more proximal locations. The spiking data were therefore converted to response coefficients by dividing the mean spike numbers obtained from stimulation at each port by the number of aesthetasc sensilla exposed to odorant via that port.

The bar graphs in Figure A.7(A) Mean response coefficients obtained from Type I local olfactory interneurons in fourteen preparations to separate stimulation via the three stimulus ports. Zone one, nearest the proximal origin of the aesthetasc array, consistently elicited the most vigorous responses to standard odorant exposures. (B) Graph of response coefficients in local olfactory interneurons from the same fourteen different preparations against Z, a dimensionless variable which varies between 0 and 1 as a function of distance along the aesthetasc array from its origin to its termination at the flagellum tip. Responses from each 


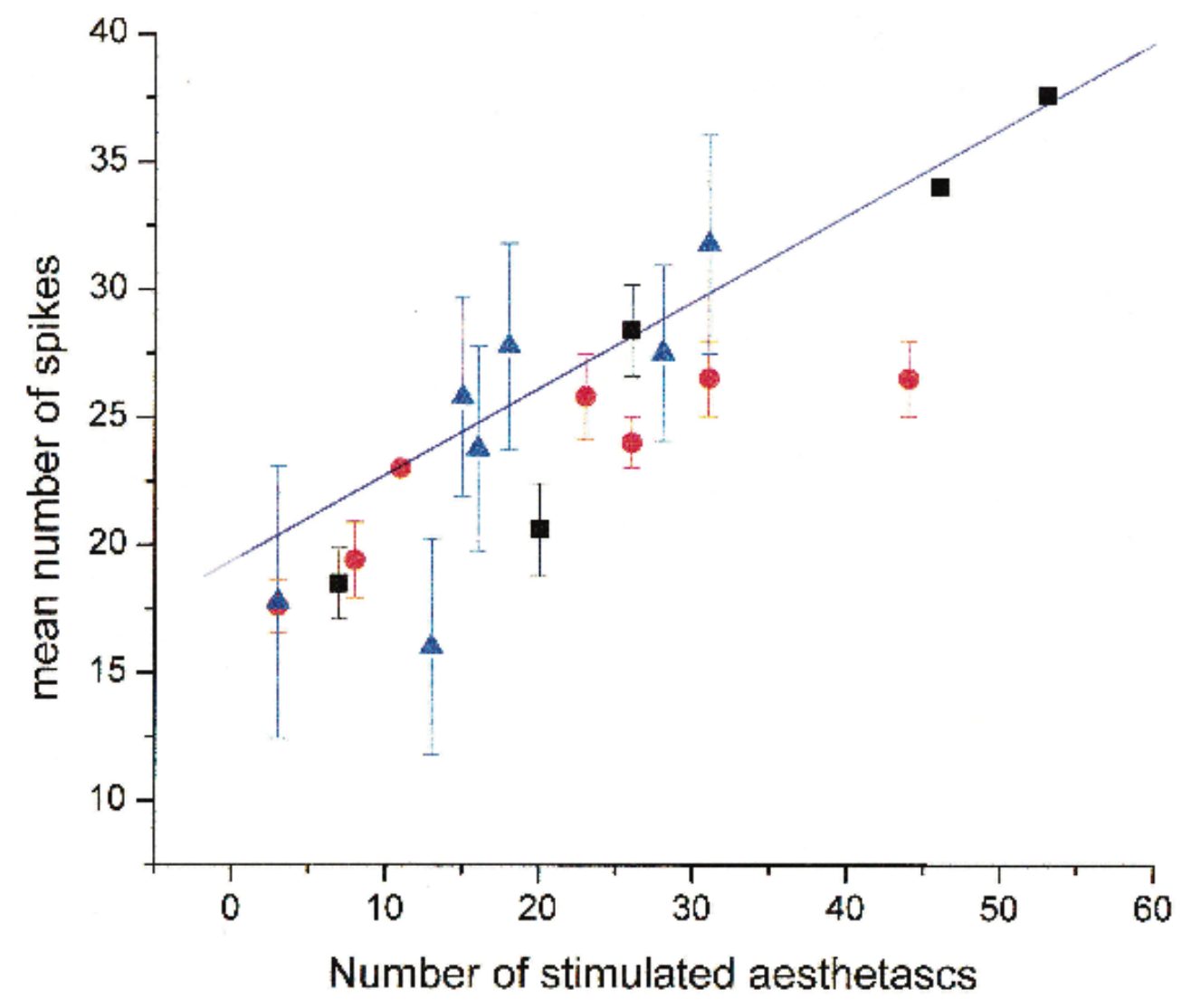

Figure A.6: Spiking responses of three Type I interneurons to different numbers of stimulated aesthetascs, including those exposed by stimulus pulses in ports $1,2,3$ and $1+2,1+3,2+3$ and $1+2+3$. The responses of individual Type I cells are indicated by the different colored symbols. The regression curve represents best linear fit of the combined data; it was calculated by least squares regression analysis and is represented by the equation $y=0.41 x+18.57$. Pearsons $r$ is 0.87 and $\mathrm{P}>0.0001$. 
Table A.1: Spiking responses to zone - and zonal combination stimulation by odorant. Mean spike numbers (APs) \pm 1 S.E. (SE) in response to stimulation of different numbers of aesthetascs (Aes) occurring within single zones or zonal combinations in five preparations, identified by bold-numbered dates above the columns, in addition to those graphed in Figure A.7(A) Mean response coefficients obtained from Type I local olfactory interneurons in fourteen preparations to separate stimulation via the three stimulus ports. Zone one, nearest the proximal origin of the aesthetasc array, consistently elicited the most vigorous responses to standard odorant exposures. (B) Graph of response coefficients in local olfactory interneurons from the same fourteen different preparations against $Z$, a dimensionless variable which varies between 0 and 1 as a function of distance along the aesthetasc array from its origin to its termination at the flagellum tip. Responses from each preparation are represented here by a different colored symbol; data points in most cases are the mean of 5 -10 stimulus trials $\pm 1 \mathrm{~S}$.E. The data are satisfactorily fit by a first-order exponential decay curve defined by the equation $Y=6.67 e^{-X / .21}+0.27$. Nonlinear regression analysis on this functional model yielded $r^{2}=0.6$ and $\mathrm{P}<0.0001$.figure.caption.67. Pearsons $\mathrm{r}$ values were obtained by linear regression analysis. Values of $\mathrm{P}$ were calculated using software at www.danielsoper.com/statcalc3/calc.aspx?id $=44$.

\begin{tabular}{|c|c|c|c|c|c|c|c|c|c|c|c|c|c|c|c|}
\hline & \multicolumn{3}{|c|}{$04 / 22 / 12(\mathrm{~A})$} & \multicolumn{3}{|c|}{$04 / 22 / 12(\mathrm{~B})$} & \multicolumn{3}{c|}{$05 / 24 / 12$} & \multicolumn{3}{c|}{$05 / 30 / 12$} & \multicolumn{3}{c|}{$04 / 12 / 12$} \\
\hline Ports & Aes & APs & SE & Aes & APs & SE & Aes & APs & SE & Aes & APs & SE & Aes & APs & SE \\
\hline 1 & 14 & 9.7 & 0.7 & 14 & 6.2 & 0.86 & 1 & 10.5 & 1.95 & 8 & 17.6 & 0.71 & 7 & 18.5 & 1.97 \\
\hline 2 & 17 & 8 & 0.01 & 17 & 4.6 & 0.51 & 16 & 11.3 & 1.5 & 11 & 10.1 & 1.53 & 20 & 20.6 & 2.58 \\
\hline 3 & 18 & 5.3 & 1.2 & 18 & 5 & 0.32 & 17 & 5.4 & 1.42 & 1 & 1.7 & 0.45 & 26 & 28.4 & 2.43 \\
\hline $1+2$ & 31 & 13.3 & 0.33 & 31 & 8 & 0.55 & 17 & 19.8 & 2.61 & 19 & 21.4 & 1.02 & 27 & 37 & 2 \\
\hline $1+3$ & 32 & 12.7 & 0.33 & 32 & 7.8 & 0.49 & 18 & 19.4 & 2.2 & 9 & 23.3 & 0.47 & 33 & 29 & 2 \\
\hline $2+3$ & 35 & 7.3 & 0.33 & 35 & 4.75 & 0.48 & 33 & 16.7 & 2.55 & 12 & 14.8 & 1.32 & 46 & 34 & 2 \\
\hline $1+2+3$ & 49 & 13.3 & 0.33 & 49 & 8.6 & 0.56 & 34 & 19.4 & 2.66 & 20 & 21.3 & 0.75 & 53 & 37.6 & 1.7 \\
\hline $\mathrm{r}$ & & 0.79 & & & 0.71 & & & 0.49 & & & 0.77 & & & 0.8 & \\
\hline $\mathrm{p}$ & & 0.02 & & & 0.04 & & & 0.13 & & & 0.02 & & & 0.02 & \\
\hline
\end{tabular}

preparation are represented here by a different colored symbol; data points in most cases are the mean of 5-10 stimulus trials \pm 1 S.E. The data are satisfactorily fit by a first-order exponential decay curve defined by the equation $Y=6.67 e^{-X / .21}+0.27$. Nonlinear regression analysis on this functional model yielded $r^{2}=0.6$ and $\mathrm{P}<0.0001$.figure.caption.67A illustrate the disparities in response coefficients of the three zonal regions in the aesthetasc array. The data represent mean values obtained across fourteen preparations. Even though they do not provide details of the disparities within individual flagella, they support the conclusion that aesthetascs near the proximal origin of the sensilla array are considerably more effective in exciting Type I cells within the OL than those located within the middle or tip regions of 
the array. This conclusion is strengthened by the graphic results displayed in Figure A.7(A) Mean response coefficients obtained from Type I local olfactory interneurons in fourteen preparations to separate stimulation via the three stimulus ports. Zone one, nearest the proximal origin of the aesthetasc array, consistently elicited the most vigorous responses to standard odorant exposures. (B) Graph of response coefficients in local olfactory interneurons from the same fourteen different preparations against Z, a dimensionless variable which varies between 0 and 1 as a function of distance along the aesthetasc array from its origin to its termination at the flagellum tip. Responses from each preparation are represented here by a different colored symbol; data points in most cases are the mean of 5-10 stimulus trials \pm 1 S.E. The data are satisfactorily fit by a first-order exponential decay curve defined by the equation $Y=6.67 e^{-X / .21}+0.27$. Nonlinear regression analysis on this functional model yielded $r^{2}=0.6$ and $\mathrm{P}<0.0001$.figure.caption.67B, in which the response disparities in each of the same fourteen preparations are shown with specific reference to relative spatial position of the stimulus zone within the aesthetasc array. Here, data are plotted as mean response coefficients against position along the aesthetasc array whose respective positions are represented as dimensionless variables between 0 and 1, with 0 as the proximal margin of the array and 1 being its termination at the tip of the flagellum. The position of each data point along the horizontal axis was taken as the midpoint of that group of aesthetascs centered within either ports 1, 2, or 3 and then converted to its fractional position, z, within the array. It is clear from these data that stimulation of the proximal region of the array, near its origin, is considerably more effective in driving Type I neurons than at more distal regions.

\section{A.4 Discussion}

Our discussion of the experimental results presented above will begin with a brief review of the organization of the primary olfactory pathways in the crayfish, as currently understood. 

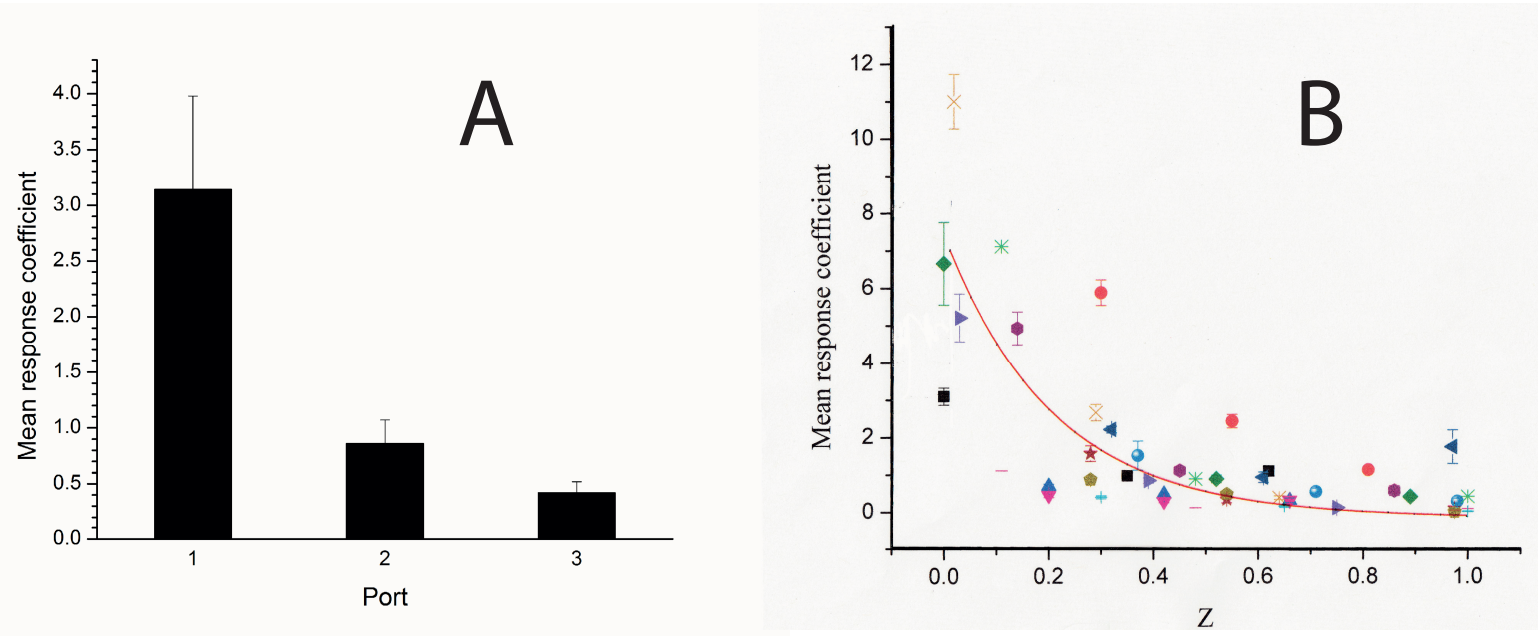

Figure A.7: (A) Mean response coefficients obtained from Type I local olfactory interneurons in fourteen preparations to separate stimulation via the three stimulus ports. Zone one, nearest the proximal origin of the aesthetasc array, consistently elicited the most vigorous responses to standard odorant exposures. (B) Graph of response coefficients in local olfactory interneurons from the same fourteen different preparations against Z, a dimensionless variable which varies between 0 and 1 as a function of distance along the aesthetasc array from its origin to its termination at the flagellum tip. Responses from each preparation are represented here by a different colored symbol; data points in most cases are the mean of 5-10 stimulus trials \pm 1 S.E. The data are satisfactorily fit by a first-order exponential decay curve defined by the equation $Y=6.67 e^{-X / .21}+0.27$. Nonlinear regression analysis on this functional model yielded $r^{2}=0.6$ and $\mathrm{P}<0.0001$.

Within each aesthetasc, dendrites of the ganglionic ORNs divide into individual microtubules, and imbedded within the microtubular membrane are olfactory receptor proteins. By inference from the distribution of olfactory receptor associations in other animals (e.g., rats, [112]; fruit flies, $[114,125])$, there is a strong suggestion that the olfactory receptor proteins associated with each specific ORN type in a sensory ganglion are unique and bind unique classes of olfactory determinants on odorant molecules. Each aesthetasc, moreover, is thought to be a heterogeneous functional replica of its neighbors. Within the primary olfactory neuropil of the crayfish brain - the paired olfactory lobes - it is thought that axon terminals of individual, class-specific ORNs from all of the antennular sensory ganglia are segregated each within their own, uniquely identifiable glomerulus, where synaptic connections are made with local interneurons, such as the Type I studied here, and with projection neurons to higher 
brain centers. In support of this putative axonal distribution pattern, uptake of radioactive tracer by ORN axons from a single pair of aesthetascs appears to be universally distributed to all ipsilateral OL glomeruli [121]. This distribution pattern of ORNs and their target central neurons resembles similar patterns in vertebrates, where ORNs target mitral cells that project to higher brain levels from the olfactory bulb and whose activity within the bulb is modulated by local interneurons, such as periglomerular neurons [119]. Spatial summation refers to the increment in postsynaptic response in consequence of the simultaneous input from several spatially separated afferent pathways (e.g., [126, 127]). Because there is presumptive evidence from recordings of local interneurons within the OL of both crayfishes [124] and spiny lobsters [128], the multiglomerular distribution of these cells and their response to complex odorants indicates that they receive inputs from different classes of ORNs, thereby satisfying the criterion of excitation via spatially different afferent pathways. The present studies with Procambarus extend this concept to include different groups of similar ORNs that are spatially distributed linearly within different aesthetascs along the antennular flagellar axis. The data presented in this paper suggest, however, that considerable functional disparity exists along the linear array of aesthetascs sensilla, and their respective ORN complements, in the stimulation of olfactory deutocerebral interneurons. Our results indicate that only about the most proximal $40 \%$ of ORNs in the crayfish, ca. $10^{4}$ in a large animal, are providing optimal excitation to the neurons within the deutocerebrum, and those ORNs within aesthetascs very close to the start of the array are dramatically the most effective. Port 1 was upstream of the majority of the sensillar array, however, and this raises concerns that the robust responses generated by stimulus pulses at this location are due to stimulus escape downstream to regions of the flagellum more densely occupied by aesthetascs. We ran controlled studies to test whether the presumed extent of zonal odorant exposure were, in fact, confined to their appropriate spatial locations. Direct observations using PLIF, indicate that the odorant exposures within the respective zones along the aesthetasc array were indeed confined to the localized flagellar sites. Furthermore, switching the three respective 
odorant supply and removal plumbing to different ports did not have any obvious effect upon the relative effectiveness of the zonal sites in activating the deutocerebral neurons. Nonetheless, questions remain about the accuracy of odorant delivery to the circumscribed zonal regions at the three stimulus ports. Escape of some odorant from port 1 during the 0.5 sec stimulus port may have spread downstream but would have been continuously diluted, encountering at its most concentrated condition a relatively low density (1-2 per annulus) of aesthetascs. The situation is very different at ports 2 and 3 , however, where the annuli individually harbor more aesthetascs (4-5 per annulus), and because the annuli are smaller, where more individual aesthetascs might be exposed to stimulus escape from the local port. This means that at ports 2 and 3, in addition to the stimulus ports exposing more aesthetascs to odorant, stimulus escape, if it occurs, would have far greater consequences in supplementing response magnitudes. Instead, the opposite appears to be true, since the response to standard stimulus pulses at these two ports was consistently, and often dramatically, less than it was at port 1. Therefore, neither PLIF experimental nor numerical considerations support the possibility that stimulus escape significantly contributed to or was responsible for the differences in stimulus efficacy at the three ports along the antennule. We conclude, therefore, that the discrepancies in response efficacy are real and must be based upon differences in the number or properties of the ORNs associated with each stimulus site or their respective central connections. A number of functional parameters could account for this. For example, disparities in the diameters or functional properties of ORN axons associated with the more distal aesthetascs would reduce the temporal density of arriving stimulus-induced action potentials at their central targets, compromising the rate of rise and/or amplitude of the resulting postsynaptic potentials. By comparison, nascent ORNs near the proximal origin of the aesthetasc array may have more uniform diameters, allowing their respective APs to present a more coherent temporal front at the central targets. Alternatively, ORNs associated with more distal aesthetascs may be functionally compromised by their age. In the crayfish Cherax destructor, it has been shown that the distal aesthetascs are the oldest on the adult 
flagellum, and even as new sensilla are being born at the proximal end of the array, those at the distal end are discarded with each molt as the 6-10 most distal annuli are shed [123], as also happens in spiny lobsters [123]. Just as in humans and other mammals, there is a periodic turnover of ORNs. It is currently unknown, however, whether existing ORNs that are not shed are replaced at each molt cycle. Another possible underlying cause of the disparity in sensillar efficacy is that synaptic connectivity by older, more distal ORN axon terminals within the OL may have different strengths or may be compromised through competition with terminals from nascent ORNs, especially if there are finite numbers of sites for synaptic contacts within the OLs. Although the size of the OLs and the numbers of local deutocerebral interneurons and projections neurons do increase in older crayfishes [129, 130], the overall number of aesthetascs and their associated ORNs also increases modestly with age despite periodic shedding of the distal annuli; this raises the possibility that synaptic competition is an ongoing feature of the olfactory pathway. Finally, whatever the cellular basis for the apparent loss in individual functional competence that occurs in the ORNs associated with the more distal aesthetascs, the effect may be somewhat offset by the continued addition of more aesthetascs in the older annuli of the antennule. Even if fewer ORNs within the aging, existing aesthetascs contribute to the detection of odorants, the continual emergence of additional aesthetascs and their compliment of nascent ORNs in more distal segments may act as a limited compensatory mechanism as they gradually migrate into the distal half of the array.

\section{A.5 Acknowledgments}

The research conducted in this study was supported by a grant from the National Science Foundation (CBET-0933034). 


\section{Appendix B}

\section{CFD model of antennule housing}

\section{chamber}

To validate the spread of odorants as it was released into the inlet ports described in Appendix A, a computer model simulated the flow of water along the central channel and odorant flow individually through each of the pairs of side channels of the antennule chamber. The flow domain inside the recording chamber was meshed into $\sim 100,000$ nodes and a transient analysis was carried out by solving the unsteady Navier-Stokes equations throughout the domain. The $\mathrm{CFX}^{\mathrm{TM}}$ solver was used to solve for water velocity and odorant concentration inside all ports of the recording chamber. The model shows that the majority of odorants that enter a channel are flushed out of the opposite channel showing no significant spread of odorants away from the port. Figure B.1Color-map of dye concentration throughout the recording chamber. The plot on the right shows odorant concentration along the centerline of the central channel.figure.caption.68 shows the colormap of odorant concentration throughout the recording chamber as well as the concentration values along the length of the central channel where the antennule would be inserted. 

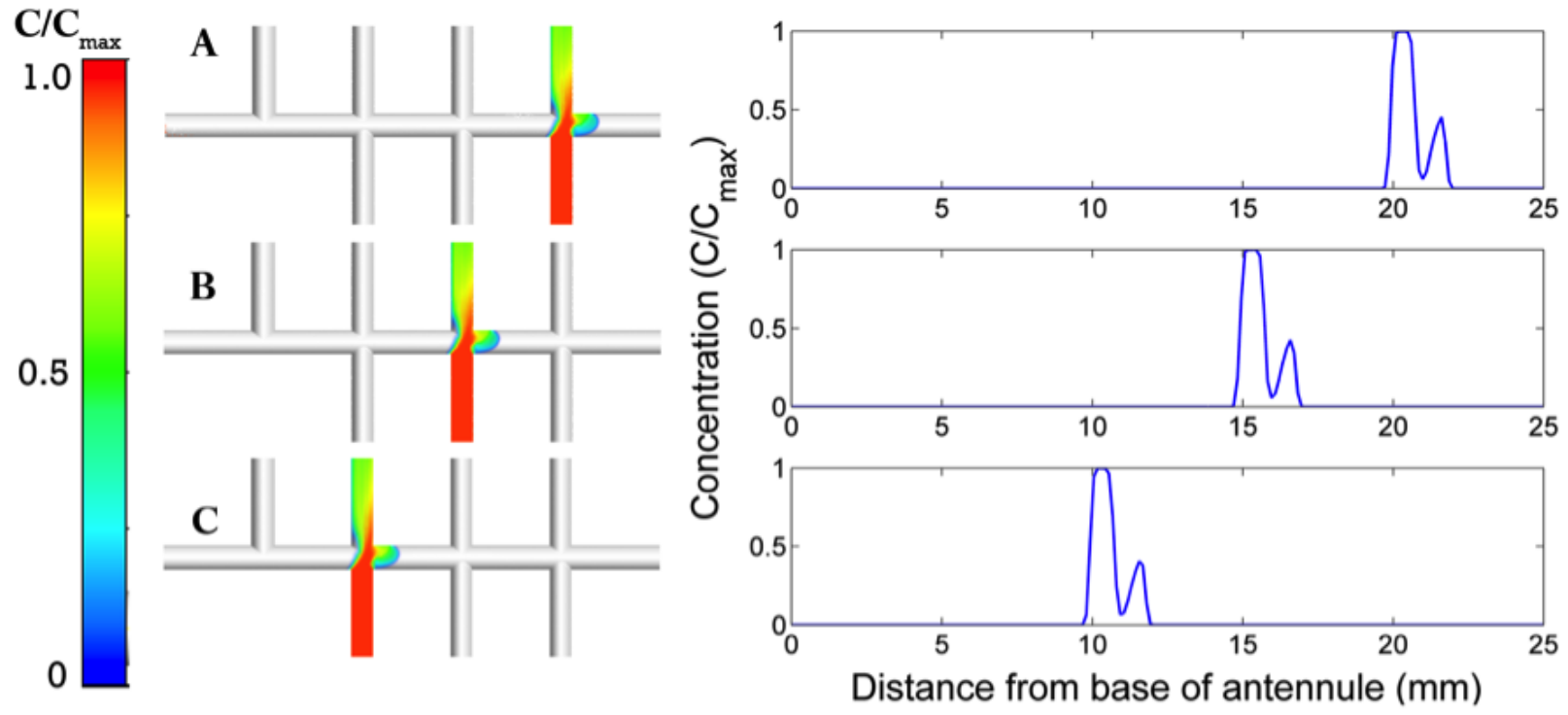

Figure B.1: Color-map of dye concentration throughout the recording chamber. The plot on the right shows odorant concentration along the centerline of the central channel. 


\section{Appendix C}

\section{Odorant diffusion over rough}

\section{topography - A two-dimensional study}

\section{C.1 Introduction}

As the odorant diffuses away from its source over rough bed topography, its increasingly intermittent and filamentous nature makes tracking its source a complex problem for organisms in that environment. A two dimensional model to study the nature of diffusing odorants over the surface of rough bed was studied to answer the following questions:

1. How do the odorant concentration, flow velocity and pressure vary as the animal moves towards the odorant source?

2. How does correlation between concentration and flow velocity change as the organism moves upstream at different elevations above the bed?

3. How are the odorant distributions and correlations effected by change in flow speed? 


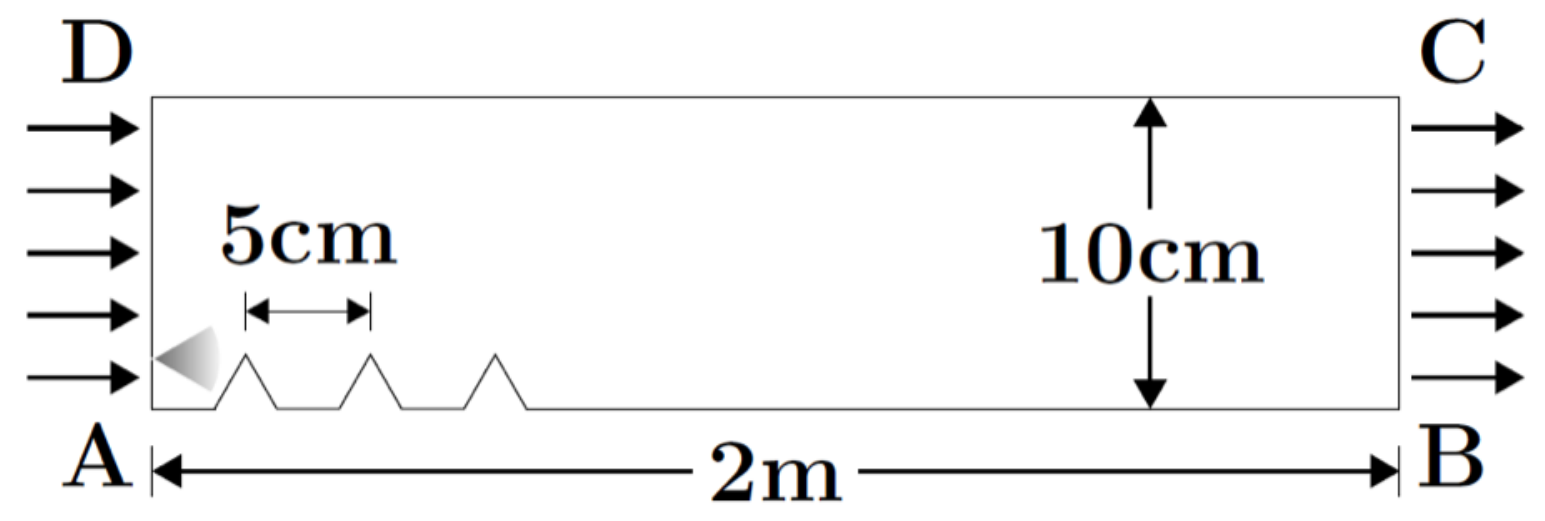

Figure C.1: Schematics of the 2D CFD model to solve for flow velocity and odorant concentrations throughout the flume.

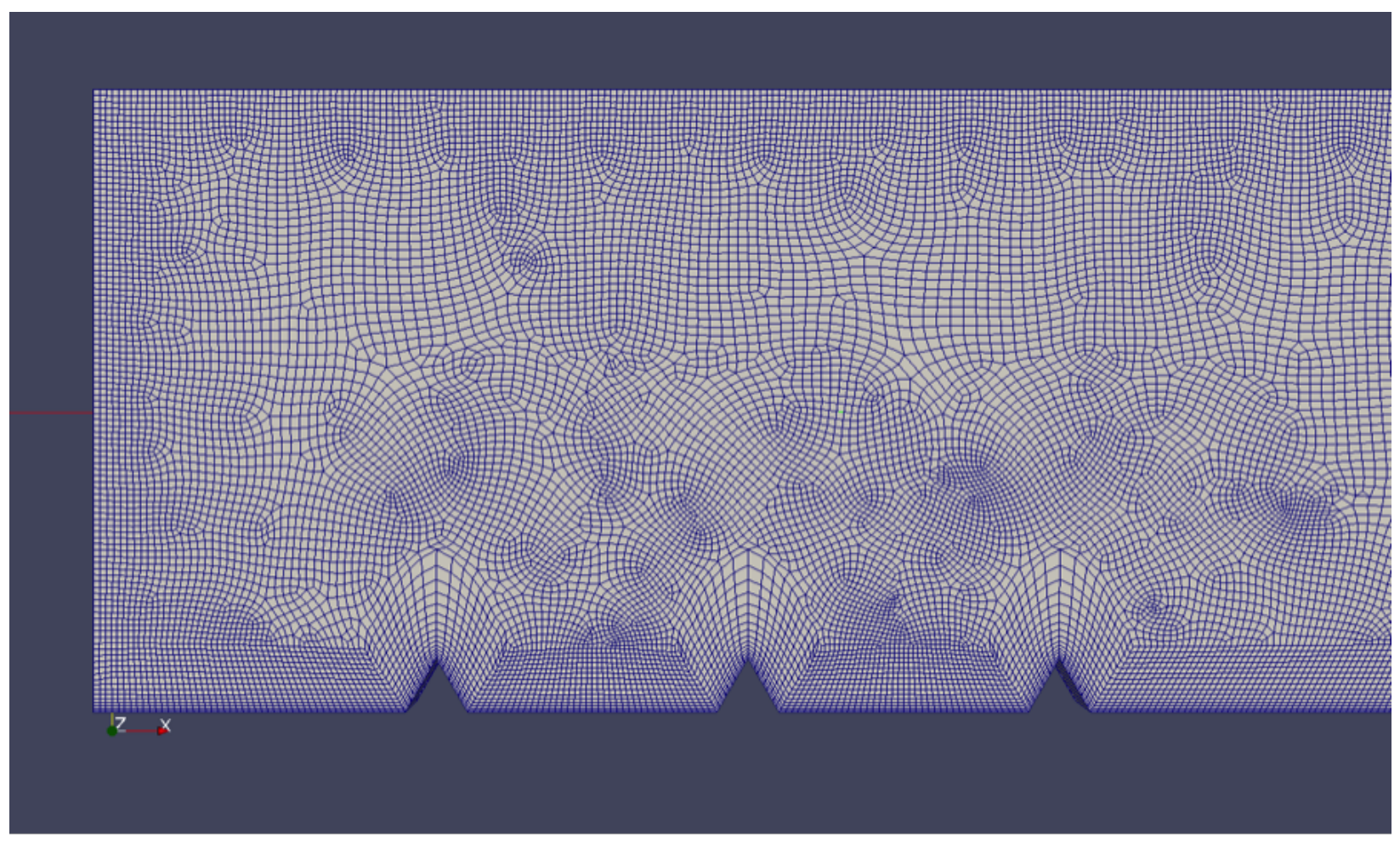

Figure C.2: Mesh for the CFD model

\section{C.2 Methods}

The simulation of the turbulent plume is carried out using a numerical model that uses triangular humps spaced at $5 \mathrm{~cm}$ from each other to simulate rough bed topography, which breaks 
the boundary layer to facilitate odorant mixing. Figure C.1Schematics of the 2D CFD model to solve for flow velocity and odorant concentrations throughout the flume.figure.caption.69 shows a rectangular flow domain where water enters the left side $\mathrm{AD}$ and exits the right side $\mathrm{BC}$ of the domain. The chemical plume enters the flow domain on the inlet side $\mathrm{AD}$ at $5 \mathrm{~mm}$ above the bed through a $2 \mathrm{~mm}$ wide opening. The grid size for the mesh is $\sim 1 \mathrm{~mm}$ which is of the order of the Kolmogorov scale $(0.76 \mathrm{~m})$ determined for the experimental flume data which enables the accurate resolution of first and second order statistics for the flow [131]. The boundary conditions for the model are the following.

- AD: water enters at uniform speed of $U_{0}(20 \mathrm{~cm} / \mathrm{s})$. The odorants enter the flow domain $5 \mathrm{~mm}$ above the bed at a normal speed of $\mathrm{U} 0$ and a concentration of $C_{\infty}\left(0.1 \mathrm{~kg} \mathrm{~m}^{-3}\right)$.

- BC: water and odorants exit the flow domain at a fixed outlet pressure of $1 \mathrm{~atm}$.

- CD: a symmetry boundary condition (free-slip walls) is applied at the top boundary of the flow domain; the normal components of the velocity and scalar variable gradients are set to zero: $u_{y}=0, \partial \Phi / \partial y=0$

- AB: no-slip wall boundary condition is applied at the bottom boundary: $u_{x}=0$ The full Navier-Stokes equation is solved for velocity, pressure and odorant concentration using the open-source software OpenFOAM ${ }^{\mathrm{TM}}$.

\section{C.3 Results}

Figure C.3Pressure, velocity and vorticity distributions around the roughness elements in the flume. Pressure units are Pascal and velocity units are $\mathrm{m} / \mathrm{s}$.figure.caption.71 shows a turbulent flow profile as water flows over the triangular humps. The humps trip up the boundary layer and facilitate mixing leading to generation of turbulent eddies as evident from the shedding vortices off the triangular humps (figure C.3Pressure, velocity and vorticity distributions around the roughness elements in the flume. Pressure units are Pascal and 
velocity units are m/s.figure.caption.71C). Figure C.4Odorant concentration color-map at a flow speed of $10 \mathrm{~cm} \mathrm{~s}^{-1}$. The units of odorant concentration are $\mathrm{kg} \mathrm{m}^{-3}$.figure.caption.72 shows the odorant concentration color map at a flow speed of $10 \mathrm{~cm} \mathrm{~s}^{-1}$. A snapshot of odorant concentrations along the centerline of the flume is shown in Figure C.5Time snapshot of odorant concentration along the length of the flume at a height of $5 \mathrm{~cm}$ from the bed.figure.caption.73. Correlation, $\rho$ between concentrations at heights of $2 \mathrm{~mm}$ and $5 \mathrm{~cm}$ are shown in Figure C.6Correlation between concentrations at heights of $2 \mathrm{~mm}$ (very close to bed) and $5 \mathrm{~cm}$ (mid-way through the flume column). The odorant concentrations at these two heights are increasingly correlated as the animal moves away from the source owing to greater vertical mixing of the odorants.figure.caption.74, as described by equation C.1Resultsequation.1.C.3.1.

$$
\rho_{c_{z 1}, c_{z 2}}=\frac{\operatorname{Cov}\left(c_{z 1}, c_{z 2}\right)}{\sigma_{c_{z 1}} \sigma_{c_{z 2}}}
$$

where $\operatorname{cov}$ is the covariance, $c_{z}$ is the concentration at height $z$ and $\sigma_{c_{z}}$ is the standard deviation of concentration at height $z$.

Increasing correlation between the concentrations at two different heights indicates greater vertical mixing of odorants as one moves away from the plume source. Figure C.7Effect of flow speed of odorant concentration distribution throughout the flume.figure.caption. 75 shows the effect of flow speed on chemical mixing and distribution throughout the flow domain. A greater degree of mixing is observed for higher flow speeds. 

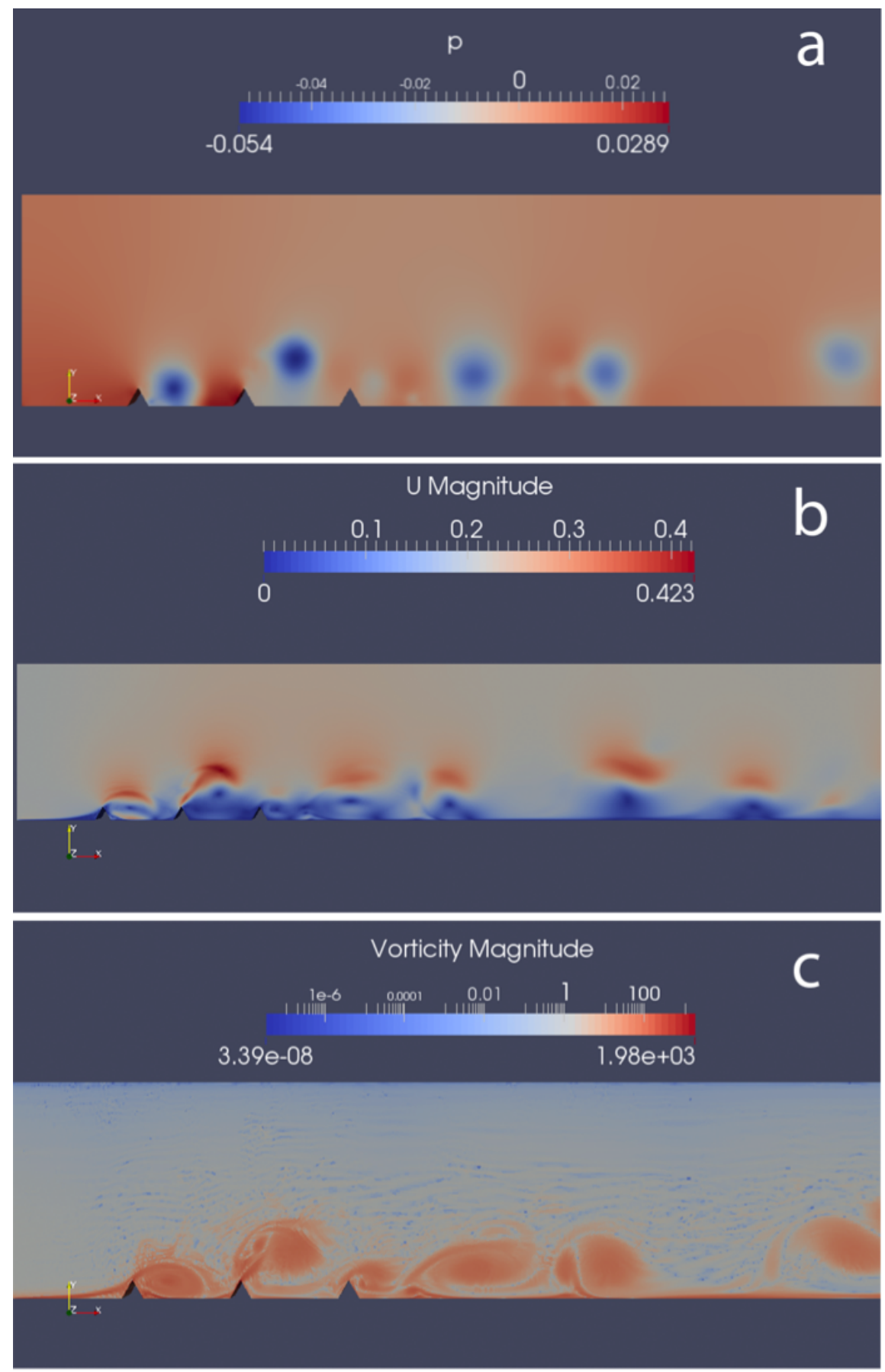

Figure C.3: Pressure, velocity and vorticity distributions around the roughness elements in the flume. Pressure units are Pascal and velocity units are $\mathrm{m} / \mathrm{s}$. 


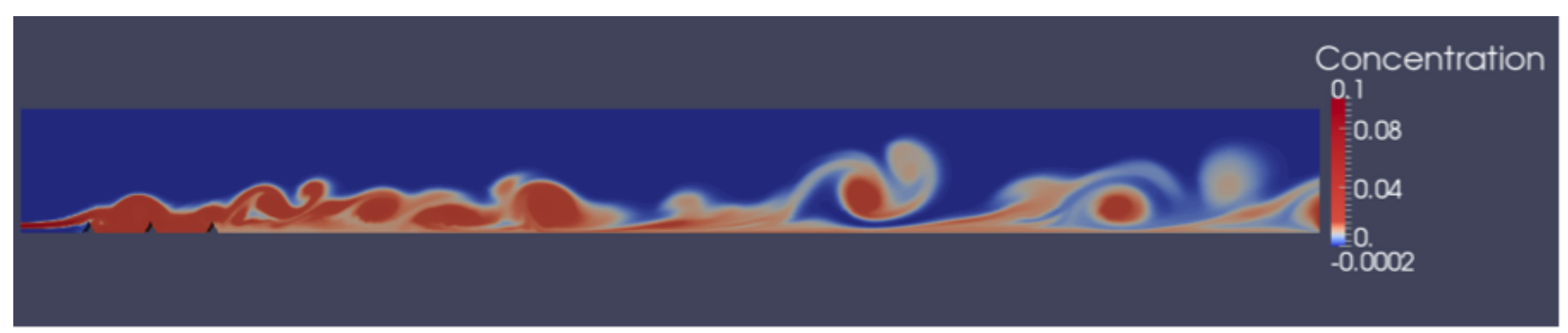

Figure C.4: Odorant concentration color-map at a flow speed of $10 \mathrm{~cm} \mathrm{~s}^{-1}$. The units of odorant concentration are $\mathrm{kg} \mathrm{m}^{-3}$.

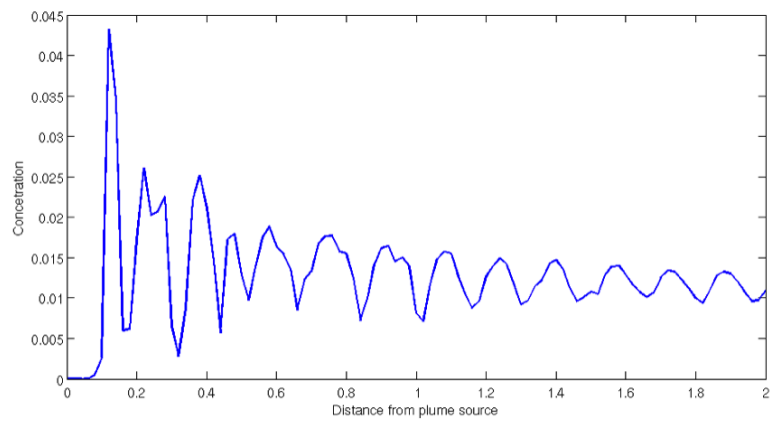

Figure C.5: Time snapshot of odorant concentration along the length of the flume at a height of $5 \mathrm{~cm}$ from the bed.

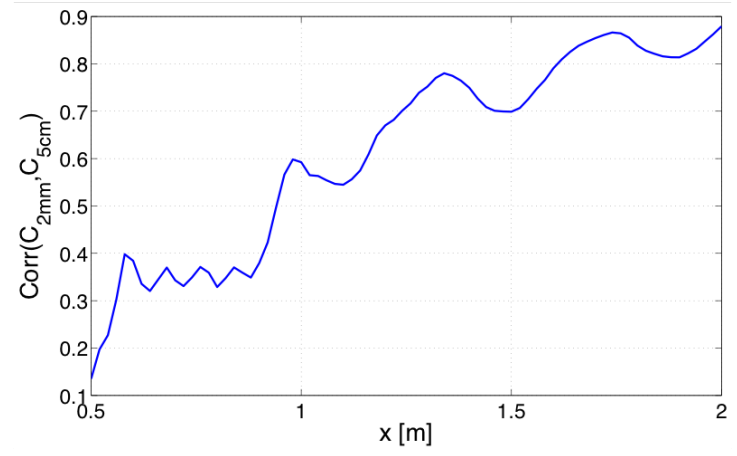

Figure C.6: Correlation between concentrations at heights of $2 \mathrm{~mm}$ (very close to bed) and 5 $\mathrm{cm}$ (mid-way through the flume column). The odorant concentrations at these two heights are increasingly correlated as the animal moves away from the source owing to greater vertical mixing of the odorants. 


\section{a. $7 \mathrm{~cm} / \mathrm{s}$}

\section{b. $10 \mathrm{~cm} / \mathrm{s}$}

Thand

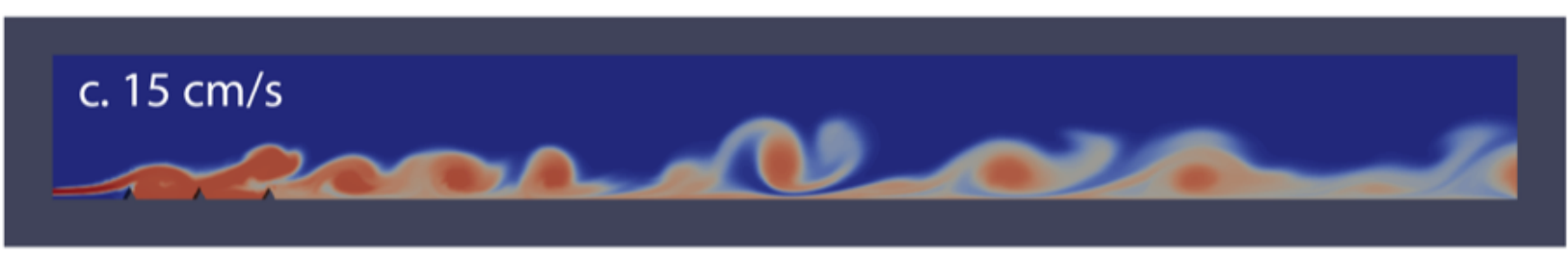
d. $20 \mathrm{~cm} / \mathrm{s}$

Figure C.7: Effect of flow speed of odorant concentration distribution throughout the flume. 


\section{Bibliography}

[1] Zimmer-Faust and Richard K. Chemical signal-to-noise detection by spiny lobsters. The Biological Bulletin, 181(3):419-426, 1991.

[2] K Berg, R Voigt, and J Atema. Flicking in the lobster homarus americanus: recordings from electrodes implanted in antennular segments. The Biological Bulletin, 183(2):377-378, 1992.

[3] Marc J Weissburg and Richard K Zimmer-Faust. Odor plumes and how blue crabs use them in finding prey. Journal of Experimental Biology, 197(1):349-375, 1994.

[4] Jelle Atema. Chemical signals in the marine environment: dispersal, detection, and temporal signal analysis. Proceedings of the National Academy of Sciences, 92(1):6266, 1995.

[5] MAR Koehl. Small-scale fluid dynamics of olfactory antennae. Marine \& Freshwater Behaviour \& Phy, 27(2-3):127-141, 1996.

[6] Mark J Weissburg. The fluid dynamical context of chemosensory behavior. The Biological Bulletin, 198(2):188-202, 2000.

[7] DeForest Mellon. Smelling, feeling, tasting and touching: Behavioral and neural integration of antennular chemosensory and mechanosensory inputs in the crayfish. The Journal of experimental biology, 215(13):2163-2172, 2012.

[8] DR Webster and MJ Weissburg. The hydrodynamics of chemical cues among aquatic organisms. Annual Review of Fluid Mechanics, 41:73-90, 2009.

[9] DeForest Mellon, H Robert Tuten, and Jan Redick. Distribution of radioactive leucine following uptake by olfactory sensory neurons in normal and heteromorphic crayfish antennules. Journal of Comparative Neurology, 280(4):645-662, 1989.

[10] Joey M Nelson, DeForest Mellon, and Matthew A Reidenbach. Effects of antennule morphology and flicking kinematics on flow and odor sampling by the freshwater crayfish, procambarus clarkii. Chemical senses, 38(8):729-741, 2013.

[11] MAR Koehl. The fluid mechanics of arthropod sniffing in turbulent odor plumes. Chemical senses, 31(2):93-105, 2006. 


\section{BIBLIOGRAPHY}

[12] Swapnil Pravin, DeForest Mellon Jr, and Matthew A Reidenbach. Micro-scale fluid and odorant transport to antennules of the crayfish, procambarus clarkii. Journal of Comparative Physiology A, 198(9):669-681, 2012.

[13] Mark T Stacey, Kristina S Mead, and Mimi AR Koehl. Molecule capture by olfactory antennules: mantis shrimp. Journal of mathematical biology, 44(1):1-30, 2002.

[14] Matthew A Reidenbach and MAR Koehl. The spatial and temporal patterns of odors sampled by lobsters and crabs in a turbulent plume. The Journal of experimental biology, 214(18):3138-3153, 2011.

[15] John P Crimaldi, Megan B Wiley, and Jeffrey R Koseff. The relationship between mean and instantaneous structure in turbulent passive scalar plumes. J. Turbul, 3(014):1-24, 2002.

[16] S Rahman and DR Webster. The effect of bed roughness on scalar fluctuations in turbulent boundary layers. Experiments in Fluids, 38(3):372-384, 2005.

[17] Marc Weissburg, Lorin Atkins, Kimberly Berkenkamp, and Danielle Mankin. Dine or dash? turbulence inhibits blue crab navigation in attractive-aversive odor plumes by altering signal structure encoded by the olfactory pathway. The Journal of experimental biology, 215(23):4175-4182, 2012.

[18] Thomas V Getchell, Thomas V and Marilyn L Getchell. Early events in vertebrate olfaction. Chemical Senses, 2(3):313-326, 1977.

[19] John A DeSimone. Physicochemical principles in taste and olfaction. Biochemistry of Taste and Olfaction, pages 213-229, 1981.

[20] Robert B Nachbar and Thomas Hellman Morton. A gas chromatographic (glpc) model for the sense of smell. variation of olfactory sensitivity with conditions of stimulation. Journal of theoretical biology, 89(3):387-407, 1981.

[21] Paul A Moore, Greg A Gerhardt, and Jelle Atema. High resolution spatio-temporal analysis of aquatic chemical signals using microelectrochemical electrodes. Chemical Senses, 14(6):829-840, 1989.

[22] J Boeckh, KE Kaissling, and D Schneider. Insect olfactory receptors. In Cold Spring Harbor Symposia on Quantitative Biology, volume 30, pages 263-280. Cold Spring Harbor Laboratory Press, 1965.

[23] RP Futrelle. How molecules get to their detectors: the physics of diffusion of insect pheromones. Trends in Neurosciences, 7(4):116-120, 1984.

[24] RW Mankin and MS Mayer. The insect antenna is not a molecular sieve. Experientia, 40(11):1251-1252, 1984.

[25] G Adam and M Delbrück. Reduction of dimensionality in biological diffusion processes. Structural chemistry and molecular biology, 198, 1968. 


\section{BIBLIOGRAPHY}

[26] Howard C Berg and Edward M Purcell. Physics of chemoreception. Biophysical journal, 20(2):193, 1977.

[27] James Dickson Murray. Lectures on nonlinear-differential-equation models in biology. Clarendon Press Oxford, 1977.

[28] R Schuech, MT Stacey, MF Barad, and MAR Koehl. Numerical simulations of odorant detection by biologically inspired sensor arrays. Bioinspiration $\&$ biomimetics, 7(1):016001, 2012.

[29] Matthew A Reidenbach, Nicole George, and MAR Koehl. Antennule morphology and flicking kinematics facilitate odor sampling by the spiny lobster, panulirus argus. Journal of Experimental Biology, 211(17):2849-2858, 2008.

[30] DeForest Mellon Jr. Physiological characterization of antennular flicking reflexes in the crayfish. Journal of Comparative Physiology A, 180(5):553-565, 1997.

[31] DeForest Mellon and Kate Christison-Lagay. A mechanism for neuronal coincidence revealed in the crayfish antennule. Proceedings of the National Academy of Sciences, 105(38):14626-14631, 2008.

[32] DeForest Mellon and Omer A Abdul Hamid. Identified antennular near-field receptors trigger reflex flicking in the crayfish. The Journal of experimental biology, 215(9):1559$1566,2012$.

[33] Harold M Monteclaro, Kazuhiko Anraku, and Tatsuro Matsuoka. Response properties of crayfish antennules to hydrodynamic stimuli: functional differences in the lateral and medial flagella. The Journal of experimental biology, 213(21):3683-3691, 2010.

[34] Jelle Atema. Eddy chemotaxis and odor landscapes: exploration of nature with animal sensors. Biological Bulletin, pages 129-138, 1996.

[35] Frank W Grasso and Jennifer A Basil. How lobsters, crayfishes, and crabs locate sources of odor: current perspectives and future directions. Current opinion in neurobiology, 12(6):721-727, 2002.

[36] Paul Moore and John Crimaldi. Odor landscapes and animal behavior: tracking odor plumes in different physical worlds. Journal of marine systems, 49(1):55-64, 2004.

[37] Amy J Horner, Manfred Schmidt, Donald H Edwards, and Charles D Derby. Role of the olfactory pathway in agonistic behavior of crayfish, procambarus clarkii. Invertebrate Neuroscience, 8(1):11-18, 2008.

[38] Troy A Keller, Ian Powell, and Marc J Weissburg. Role of olfactory appendages in chemically mediated orientation of blue crabs. Marine Ecology Progress Series, 261:217-231, 2003.

[39] Meg E Johnson and Jelle Atema. The olfactory pathway for individual recognition in the american lobster homarus americanus. Journal of experimental biology, 208(15):2865-2872, 2005. 


\section{BIBLIOGRAPHY}

[40] Barbara S Beltz, Kashka Kordas, Monaya M Lee, Jennifer B Long, Jeanne L Benton, and David C Sandeman. Ecological, evolutionary, and functional correlates of sensilla number and glomerular density in the olfactory system of decapod crustaceans. Journal of Comparative Neurology, 455(2):260-269, 2003.

[41] Barry W Ache and Janet M Young. Olfaction: diverse species, conserved principles. Neuron, 48(3):417-30, Nov 2005.

[42] DR Webster and MJ Weissburg. Chemosensory guidance cues in a turbulent chemical odor plume. Limnology and Oceanography, 46(5):1034-1047, 2001.

[43] JP Crimaldi and JR Koseff. High-resolution measurements of the spatial and temporal scalar structure of a turbulent plume. Experiments in Fluids, 31(1):90-102, 2001.

[44] Paul A Moore and Jennifer L Grills. Chemical orientation to food by the crayfishi ii orconectes rusticusi/ij: influence of hydrodynamics. Animal Behaviour, 58(5):953-963, 1999.

[45] MAR Koehl, Jeffrey R Koseff, John P Crimaldi, Michael G McCay, Tim Cooper, Megan B Wiley, and Paul A Moore. Lobster sniffing: antennule design and hydrodynamic filtering of information in an odor plume. Science, 294(5548):1948-1951, 2001.

[46] Mimi AR Koehl. Hydrodynamics of sniffing by crustaceans. In Chemical communication in crustaceans, pages 85-102. Springer, 2011.

[47] Brian C Schmitt and Barry W Ache. Olfaction: responses of a decapod crustacean are enhanced by flicking. Science, 205(4402):204-206, 1979.

[48] Joseph AC Humphrey and DeForest Mellon. Analytical and numerical investigation of the flow past the lateral antennular flagellum of the crayfish procambarus clarkii. Journal of Experimental Biology, 210(17):2969-2978, 2007.

[49] DeForest Mellon and Joseph AC Humphrey. Directional asymmetry in responses of local interneurons in the crayfish deutocerebrum to hydrodynamic stimulation of the lateral antennular flagellum. Journal of Experimental Biology, 210(17):2961-2968, 2007.

[50] NE Holden and DR Lide. Crc handbook of chemistry and physics, 1991.

[51] Mark W Denny. Air and water: the biology and physics of life's media. Princeton University Press, 1993.

[52] William H Bossert and Edward O Wilson. The analysis of olfactory communication among animals. Journal of theoretical biology, 5(3):443-469, 1963.

[53] Neil J Vickers. Winging it: moth flight behavior and responses of olfactory neurons are shaped by pheromone plume dynamics. Chemical senses, 31(2):155-166, 2006. 


\section{BIBLIOGRAPHY}

[54] Marc J Weissburg and Richard K Zimmer-Faust. Life and death in moving fluids: hydrodynamic effects on chemosensory-mediated predation. Ecology, 74(5):1428-1443, 1993.

[55] JL Jackson, DR Webster, S Rahman, and MJ Weissburg. Bed roughness effects on boundary-layer turbulence and consequences for odor-tracking behavior of blue crabs (callinectes sapidus). Limnology and Oceanography, 52(5):1883-1897, 2007.

[56] Kristina S Mead, Megan B Wiley, MAR Koehl, and Jeffrey R Koseff. Fine-scale patterns of odor encounter by the antennules of mantis shrimp tracking turbulent plumes in wave-affected and unidirectional flow. Journal of Experimental Biology, 206(1):181-193, 2003.

[57] Jennifer L Page, Brian D Dickman, Donald R Webster, and Marc J Weissburg. Getting ahead: context-dependent responses to odorant filaments drive along-stream progress during odor tracking in blue crabs. The Journal of experimental biology, 214(9):1498-1512, 2011.

[58] Charles D Derby, Pascal Steullet, Amy J Horner, and Holly S Cate. The sensory basis of feeding behaviour in the caribbean spiny lobster, panulirus argus. Marine and Freshwater Research, 52(8):1339-1350, 2001.

[59] Rachel I Wilson. Neural and behavioral mechanisms of olfactory perception. Current opinion in neurobiology, 18(4):408-412, 2008.

[60] Chih-Ying Su, Karen Menuz, and John R Carlson. Olfactory perception: receptors, cells, and circuits. Cell, 139(1):45-59, 2009.

[61] GEORGE Gomez and JELLE Atema. Temporal resolution in olfaction ii: time course of recovery from adaptation in lobster chemoreceptor cells. Journal of neurophysiology, 76(2):1340-1351, 1996.

[62] HP Marschall and BW Ache. Response dynamics of lobster olfactory neurons during simulated natural sampling. Chem. Senses, 14:725, 1989.

[63] E. Zettler and J. Atema. Chemoreceptor cells as concentration slope detectors: Preliminary evidence from the lobster nose. The Biological Bulletin, 197(2):252-253, 1999.

[64] G Gomez, R Voigt, and J Atema. Temporal resolution in olfaction iii: flicker fusion and concentration-dependent synchronization with stimulus pulse trains of antennular chemoreceptor cells in the american lobster. Journal of Comparative Physiology A, 185(5):427-436, 1999.

[65] Paola F Borroni and Jelle Atema. Adaptation in chemoreceptor cells. Journal of Comparative Physiology A, 164(1):67-74, 1988. 


\section{BIBLIOGRAPHY}

[66] GEORGE Gomez and JELLE Atema. Temporal resolution in olfaction: stimulus integration time of lobster chemoreceptor cells. The Journal of experimental biology, 199(8):1771-1779, 1996.

[67] AYL Cheer and MAR Koehl. Fluid flow through filtering appendages of insects. Mathematical Medicine and Biology, 4(3):185-199, 1987.

[68] DeForest Mellon. Integration of hydrodynamic and odorant inputs by local interneurons of the crayfish deutocerebrum. Journal of experimental biology, 208(19):3711$3720,2005$.

[69] Marc J Weissburg, Matthew C Ferner, Daniel P Pisut, and Delbert L Smee. Ecological consequences of chemically mediated prey perception. Journal of Chemical Ecology, 28(10):1953-1970, 2002.

[70] Troy A Keller and Marc J Weissburg. Effects of odor flux and pulse rate on chemosensory tracking in turbulent odor plumes by the blue crab, callinectes sapidus. The Biological Bulletin, 207(1):44-55, 2004.

[71] Rachel I Wilson. Neural and behavioral mechanisms of olfactory perception. Curr Opin Neurobiol, 18(4):408-12, Aug 2008.

[72] Jennifer L Page, Brian D Dickman, Donald R Webster, and Marc J Weissburg. Staying the course: chemical signal spatial properties and concentration mediate crossstream motion in turbulent plumes. The Journal of experimental biology, 214(9):1513$1522,2011$.

[73] DeForest Mellon Jr and Matthew A Reidenbach. Frontiers in Sensing Systems, chapter Fluid mechanical problems in crustacean active chemoreception. Springer, 2012.

[74] Miranda L Wilson and Marc J Weissburg. Temporal and spatial sampling strategies maintain tracking success of whelks to prey patches of differing distributions. Animal Behaviour, 84(6):1323-1330, 2012.

[75] DeForest Mellon. Regulation of conduction velocity in axons from near-field receptors of the crayfish antennule. The Journal of experimental biology, 213(22):3778-3786, 2010 .

[76] Jayne M Gardiner and Jelle Atema. Sharks need the lateral line to locate odor sources: rheotaxis and eddy chemotaxis. Journal of Experimental Biology, 210(11):1925-1934, 2007.

[77] Frank W Grasso and Jelle Atema. Integration of flow and chemical sensing for guidance of autonomous marine robots in turbulent flows. Environmental Fluid Mechanics, 2(1-2):95-114, 2002. 


\section{BIBLIOGRAPHY}

[78] Holly S Cate and Charles D Derby. Morphology and distribution of setae on the antennules of the caribbean spiny lobster panulirus argus reveal new types of bimodal chemo-mechanosensilla. Cell and tissue research, 304(3):439-454, 2001.

[79] Mary C Wolf, Rainer Voigt, and Paul A Moore. Spatial arrangement of odor sources modifies the temporal aspects of crayfish search strategies. Journal of chemical ecology, 30(3):501-517, 2004.

[80] Richard K Zimmer-Faust, Christopher M Finelli, N Dean Pentcheff, and David S Wethey. Odor plumes and animal navigation in turbulent water flow: a field study. The Biological Bulletin, 188(2):111-116, 1995.

[81] MA Reidenbach, M Limm, M Hondzo, and MT Stacey. Effects of bed roughness on boundary layer mixing and mass flux across the sediment-water interface. Water Resources Research, 46(7), 2010.

[82] JP Crimaldi. Planar laser induced fluorescence in aqueous flows. Experiments in fluids, 44(6):851-863, 2008.

[83] M Raffel, C Willert, and J Kompenhans. Particle image velocimetry: a practical guide,(1998).

[84] EA Cowen, K-A Chang, and Q Liao. A single-camera coupled ptv-lif technique. Experiments in fluids, 31(1):63-73, 2001.

[85] EA Cowen and SG Monismith. A hybrid digital particle tracking velocimetry technique. Experiments in Fluids, 22(3):199-211, 1997.

[86] J Kristian Sveen and Edwin A Cowen. Quantitative imaging techniques and their application to wavy flows. Advances in Coastal and Ocean Engineering, 9:1, 2004.

[87] Anthony Edward Perry, William H Schofield, and Peter N Joubert. Rough wall turbulent boundary layers. Journal of Fluid Mechanics, 37(02):383-413, 1969.

[88] Christopher M Finelli, N Dean Pentcheff, Richard K Zimmer-Faust, and David S Wethey. Odor transport in turbulent flows: constraints on animal navigation. Limnology and Oceanography, 44(4):1056-1071, 1999.

[89] Jennifer Basil and Jelle Atema. Lobster orientation in turbulent odor plumes: simultaneous measurement of tracking behavior and temporal odor patterns. The Biological Bulletin, 187(2):272-273, 1994.

[90] PF Beglane, FW Grasso, JA Basil, and J Atema. Far field chemo-orientation in the american lobster, homarus americanus: effects of unilateral ablation and lesioning of the lateral antennule. The Biological Bulletin, 193(2):214-215, 1997.

[91] Jayne M Gardiner and Jelle Atema. The function of bilateral odor arrival time differences in olfactory orientation of sharks. Current Biology, 20(13):1187-1191, 2010. 


\section{BIBLIOGRAPHY}

[92] Brian D Dickman, Donald R Webster, Jennifer L Page, and Marc J Weissburg. Threedimensional odorant concentration measurements around actively tracking blue crabs. Limnol. Oceanogr. Methods, 7:96-108, 2009.

[93] Barry W Ache. Phylogeny of smell and taste, 1991.

[94] DeForest Mellon. Combining dissimilar senses: central processing of hydrodynamic and chemosensory inputs in aquatic crustaceans. The Biological Bulletin, 213(1):1-11, 2007.

[95] S. Pravin and M. A. Reidenbach. Simultaneous sampling of flow and odorants by crustaceans can aid searches within a turbulent plume. Sensors (Basel), 13(12):16591610, 2013. Pravin, Swapnil Reidenbach, Matthew A eng Research Support, U.S. Gov't, Non-P.H.S. Switzerland Basel, Switzerland 2013/12/05 06:00 Sensors (Basel). 2013 Dec 3;13(12):16591-610. doi: 10.3390/s131216591.

[96] K. S. Mead and M. A. Koehl. Stomatopod antennule design: the asymmetry, sampling efficiency and ontogeny of olfactory flicking. Journal of Experimental Biology, 203(24):3795-3808, 2000.

[97] Rebecca B Price and Barry W Ache. Peripheral modification of chemosensory information in the spiny lobster. Comparative Biochemistry and Physiology Part A: Physiology, 57(2):249-253, 1977.

[98] Holly S. Cate and Charles D. Derby. Hooded sensilla homologues: structural variations of a widely distributed bimodal chemomechanosensillum. Journal of Comparative Neurology, 444(4):345-357, 2002.

[99] Holly S. Cate and Charles D. Derby. Ultrastructure and physiology of the hooded sensillum, a bimodal chemomechanosensillum of lobsters. Journal of Comparative Neurology, 442(4):293-307, 2002.

[100] Edward S. Hodgson and Robert F. Mathewson. Chemosensory orientation in sharks. Annals of the New York Academy of Sciences, 188(1):175-181, 1971.

[101] Jeannette Yen, Marc J Weissburg, and Michael H Doall. The fluid physics of signal perception by mate-tracking copepods. Philosophical Transactions of the Royal Society of London. Series B: Biological Sciences, 353(1369):787-804, 1998.

[102] Kirsten Pohlmann, Jelle Atema, and Thomas Breithaupt. The importance of the lateral line in nocturnal predation of piscivorous catfish. Journal of Experimental Biology, 207(17):2971-2978, 2004.

[103] Kimberly B Catton, Donald R Webster, Jason Brown, and Jeannette Yen. Quantitative analysis of tethered and free-swimming copepodid flow fields. Journal of Experimental Biology, 210(2):299-310, 2007. 
[104] Ann Jane Tierney, CS Thompson, and DW Dunham. Fine structure of aesthetasc chemoreceptors in the crayfish orconectes propinquus. Canadian journal of zoology, 64(2):392-399, 1986.

[105] Ulrike Grünert and Barry W Ache. Ultrastructure of the aesthetasc (olfactory) sensilla of the spiny lobster, panulirus argus. Cell and Tissue Research, 251(1):95-103, 1988.

[106] Deforest Mellon, Swapnil Pravin, and Matthew A. Reidenbach. A nose too far: Regional differences in olfactory receptor neuron efficacy along the crayfish antennule. The Biological Bulletin, 227(1):40-50, 2014.

[107] Linda Buck and Richard Axel. A novel multigene family may encode odorant receptors: a molecular basis for odor recognition. Cell, 65(1):175-187, 1991.

[108] Kerry J Ressler, Susan L Sullivan, and Linda B Buck. A zonal organization of odorant receptor gene expression in the olfactory epithelium. Cell, 73(3):597-609, 1993.

[109] Xinmin Zhang and Stuart Firestein. The olfactory receptor gene superfamily of the mouse. Nature neuroscience, 5(2):124-133, 2002.

[110] Tamara Elmore, Rickard Ignell, John R Carlson, and Dean P Smith. Targeted mutation of a drosophila odor receptor defines receptor requirement in a novel class of sensillum. The Journal of neuroscience, 23(30):9906-9912, 2003.

[111] Aaron L Goldman, Wynand Van der Goes van Naters, Derek Lessing, Coral G Warr, and John R Carlson. Coexpression of two functional odor receptors in one neuron. Neuron, 45(5):661-666, 2005.

[112] Peter Mombaerts, Fan Wang, Catherine Dulac, Steve K Chao, Adriana Nemes, Monica Mendelsohn, James Edmondson, and Richard Axel. Visualizing an olfactory sensory map. Cell, 87(4):675-686, 1996.

[113] Joseph L Dynes and John Ngai. Pathfinding of olfactory neuron axons to stereotyped glomerular targets revealed by dynamic imaging in living zebrafish embryos. Neuron, 20(6):1081-1091, 1998.

[114] Leslie B Vosshall, Hubert Amrein, Pavel S Morozov, Andrey Rzhetsky, and Richard Axel. A spatial map of olfactory receptor expression in thei $i j$ drosophilai/i $i$ antenna. Cell, 96(5):725-736, 1999.

[115] Zhihua Zou, Lisa F Horowitz, Jean-Pierre Montmayeur, Scott Snapper, and Linda B Buck. Genetic tracing reveals a stereotyped sensory map in the olfactory cortex. Nature, 414(6860):173-179, 2001.

[116] Elissa A Hallem, Michael G Ho, and John R Carlson. The molecular basis of odor coding in thei ii drosophilai/ii, antenna. Cell, 117(7):965-979, 2004.

[117] I Boekhoff, WC Michel, H Breer, and BW Ache. Single odors differentially stimulate dual second messenger pathways in lobster olfactory receptor cells. The Journal of neuroscience, 14(5):3304-3309, 1994. 


\section{BIBLIOGRAPHY}

[118] F Zufall, S Firestein, and GM Shepherd. Cyclic nucleotide-gated ion channels and sensory transduction in olfactory receptor neurons. Annual review of biophysics and biomolecular structure, 23(1):577-607, 1994.

[119] John G Hildebrand and Gordon M Shepherd. Mechanisms of olfactory discrimination: converging evidence for common principles across phyla. Annual review of neuroscience, 20(1):595-631, 1997.

[120] David C Sandeman and Jeffrey L Denburg. The central projections of chemoreceptor axons in the crayfish revealed by axoplasmic transport. Brain research, 115(3):492496, 1976.

[121] DeForest Mellon and Steven D Munger. Nontopographic projection of olfactory sensory neurons in the crayfish brain. Journal of Comparative Neurology, 296(2):253262, 1990.

[122] Eric Hallberg, Kjell UI Johansson, and Rolf Elofsson. The aesthetasc concept: structural variations of putative olfactory receptor cell complexes in crustacea. Microscopy research and technique, 22(4):325-335, 1992.

[123] R Sandeman and D Sandeman. Pre-and postembryonic development, growth and turnover of olfactory receptor neurones in crayfish antennules. The Journal of experimental biology, 199(11):2409-2418, 1996.

[124] D Mellon Jr and V Alones. Identification of three classes of multiglomerular, broadspectrum neurons in the crayfish olfactory midbrain by correlated patterns of electrical activity and dendritic arborization. Journal of Comparative Physiology A, 177(1):55-71, 1995.

[125] Leslie B Vosshall, Allan M Wong, and Richard Axel. An olfactory sensory map in the fly brain. Cell, 102(2):147-159, 2000.

[126] Charles S Sherrington. Remarks on some aspects of reflex inhibition. Proceedings of the Royal Society of London. Series B, Containing Papers of a Biological Character, pages 519-545, 1925.

[127] John Carew Eccles et al. The physiology of nerve cells. 1957.

[128] M Schmidt and BW Ache. Processing of antennular input in the brain of the spiny lobster, panulirus argus. Journal of Comparative Physiology A, 178(5):605-628, 1996.

[129] Manfred Schmidt and DeForest Mellon Jr. Neuronal processing of chemical information in crustaceans. In Chemical communication in crustaceans, pages 123-147. Springer, 2011.

[130] Barbara S Beltz, Yi Zhang, Jeanne L Benton, and David C Sandeman. Adult neurogenesis in the decapod crustacean brain: a hematopoietic connection? European Journal of Neuroscience, 34(6):870-883, 2011. 


\section{BIBLIOGRAPHY}

[131] Parviz Moin and Krishnan Mahesh. Direct numerical simulation: a tool in turbulence research. Annual Review of Fluid Mechanics, 30(1):539-578, 1998. 